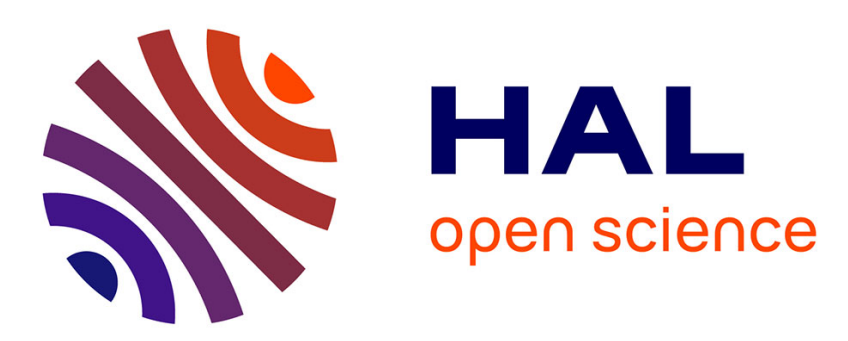

\title{
Functional characterisation of naturally occurring genetic variants in the human TLR1-2-6 gene family
}

Meriem Ben-Ali, Beatrice Corre, Jeremy Manry, Luis Barreiro, Hélène Quach, Michele Boniotto, Sandra Pellegrini, Lluis Quintana-Murci

\section{- To cite this version:}

Meriem Ben-Ali, Beatrice Corre, Jeremy Manry, Luis Barreiro, Hélène Quach, et al.. Functional characterisation of naturally occurring genetic variants in the human TLR1-2-6 gene family. Human Mutation, 2011, 32 (6), pp.643-52. 10.1002/humu.21486 . hal-00644710

\author{
HAL Id: hal-00644710 \\ https://hal.science/hal-00644710
}

Submitted on 25 Nov 2011

HAL is a multi-disciplinary open access archive for the deposit and dissemination of scientific research documents, whether they are published or not. The documents may come from teaching and research institutions in France or abroad, or from public or private research centers.
L'archive ouverte pluridisciplinaire HAL, est destinée au dépôt et à la diffusion de documents scientifiques de niveau recherche, publiés ou non, émanant des établissements d'enseignement et de recherche français ou étrangers, des laboratoires publics ou privés. 
Human Mutation

WILEY

\section{Functional characterisation of naturally occurring genetic variants in the human TLR1-2-6 gene family}

\begin{tabular}{|r|l|}
\hline Journal: & Human Mutation \\
\hline Manuscript ID: & humu-2010-0516.R1 \\
\hline Wiley - Manuscript type: & Research Article \\
\hline Autho Submitted by the & 24-Jan-2011 \\
\hline Complete List of Authors: & $\begin{array}{l}\text { Ben-Ali, Meriem; Institut Pasteur, Genomes \& Genetics } \\
\text { Corre, Beatrice; Institut Pasteur, Genomes \& Genetics } \\
\text { Manry, Jeremy; Institut Pasteur, Genomes \& Genetics } \\
\text { Barreiro, Luis; University of Chicago, Department of Human } \\
\text { Genetics } \\
\text { Quach, Hélène; Institut Pasteur, Genomes \& Genetics } \\
\text { Boniotto, Michele; Institut Pasteur, Genomes \& Genetics } \\
\text { Pellegrini, Sandra; Institut Pasteur, Genomes \& Genetics } \\
\text { Quintana-Murci, Lluis; Institut Pasteur, Genomes \& Genetics }\end{array}$ \\
\hline \hline Key Words: & TLRs, innate immunity, non-synonymous mutations, NF-kB activity \\
\hline
\end{tabular}

\section{SCHOLARONE ${ }^{\text {th }}$ Manuscripts}




\section{RESPONSES TO REFEREE'S COMMENTS}

\section{Referee: 1}

The present manuscript by Meriem Ben-Ali et al analyzes the functional impact of human TLR12-6 variants with altered amino-acid sequences.

The study comprises the identification of new mutations by DNA sequencing and the subsequent functional characterisation of 41 variants with amino-acid alterations in the TLR1-2-6 family. Meriem Ben-Ali et al find 17 new sequence variations by investigating a total of 158 individuals (316 chromosomes from Sub-Saharan Africa, Europe and East-Asia). 14 variants (5 TLR1, 4 TLR2, and 5 TLR6 variants) displayed an impairment of NF- $k B$ activation in genetic complementation assays with HEK293T cells. Most of these variants are present at very low population frequencies and are population-specific.

The manuscript presents a comprehensive study dealing with the proper identification of sequence variations in genes of the innate immune system. Major points of criticism focus on the functional characterisation of these receptors.

The manuscript should only be published in Human Mutation after addressing the following points:

1-The authors can not exclude that their functionally relevant TLR-variants cause apoptosis by over-expression, explaining their results of hyporesponsiveness. Here, stimuli like TNFa (TLR-independent) should be included al least for the functionally relevant variants demonstrated.

In order to exclude the possibility that the functionally relevant TLR variants caused apoptosis upon $24 \mathrm{hr}$ over-expression, we measured the percentage of apoptotic cells present in each transfected population using 7-amino actinomycin D (7-AAD) flow cytometry staining (see Figure below). In cells transfected with the empty vector, the percentage of apoptotic cells was about $4 \%$ (negative control). Upon treatment with an inducer of apoptosis, staurosporin, $40 \%$ of the cells were apoptotic (positive control). In cells transfected with each of the 17 TLR constructs (14 functionally relevant TLR variants and the 3 ancestral forms), the percentage of apoptotic cells ranged between 3 and $8 \%$, and no correlation could be made with the level of NFKB responsiveness of the variants. On this basis, we do not think that the overexpression of our functionally relevant TLR constructs causes apoptosis.

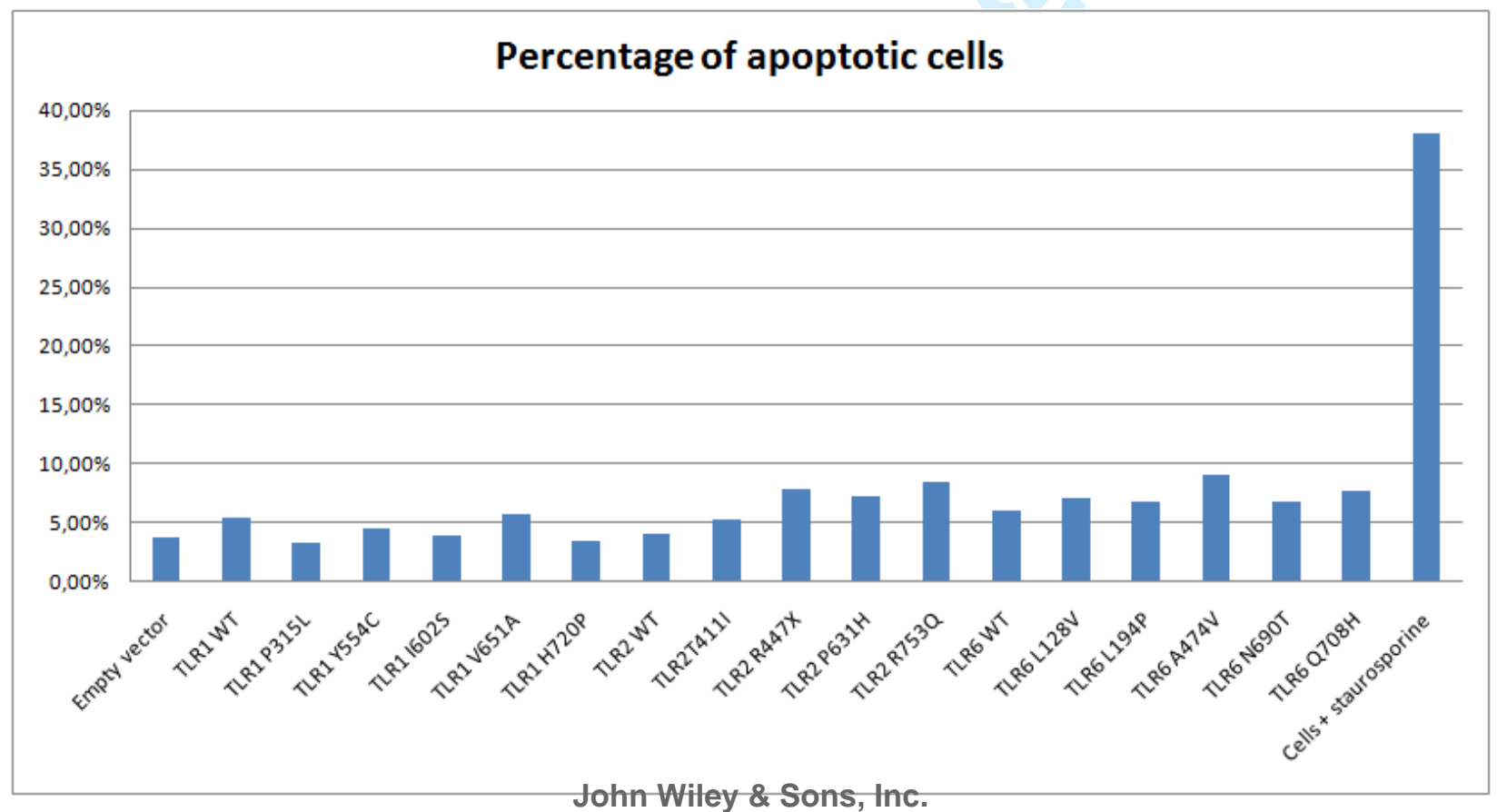


2- Other pathogen associated molecular patterns for TLR2 like peptidoglycan or zymosan were not investigated.

The reviewer is right in that it would be interesting to test other ligands of TLR2, as well as other ligands of TLR1 and TLR6. However, our goal in this study was to perform a functional comparison of a large number of naturally occurring variants in a single assay and not to dissect the effects of each single mutation upon challenge with different ligands. Given the large number of variants to be tested, we chose for each receptor the most commonly used ligand in order to make our results comparable with previous studies.

3-Most of the TLR2-data were published before (e.g. Lorenz et al 2000; Merx et al. 2007); is there a need to do it again? If so, comparable and different results to previously reported manuscripts must be discussed.

Indeed, a large part of the TLR2 variants that we have studied had been previously identified. Currently in the databases, for the human TLR2 gene there are 13 non-synonymous variants and one stop mutation. Our TLR2 resequencing effort of a panel of 158 healthy individuals originating from sub-Saharan Africa, Europe and East Asia, led us to identify 4 of these already known variants, the stop mutation as well as two previously unknown non-synonymous variants.

The aim of our study was to characterize blindly all mutations identified in our population genetics screen. Indeed, some discrepancies in the literature can be found concerning the functional impact of two TLR2 variants (T411I and P631H).

As suggested by the reviewer, in the revised manuscript, we discuss how our study may help to clarify previous discrepancies concerning TLR2 variants (see page 17)

4-The authors take cells that already express TLR6 in genetic complementation assays to analyse TLR6. They do not mention that other cells than HEK293 (e.g. CHO or NIH3T3) were investigated to solve this problem. The way to handle this problem in HEK293T is hardly described.

We agree with the reviewer that we did not describe in sufficient detail how we handled the problem of expression of endogenous TLR6 in the recipient cells. In fact, we did extensive preliminary work to set up transfection conditions and minimize the potential noise from endogenous TLR6. Specifically, the amounts of transfected TLR6 and TLR2 constructs were optimized so as to reduce reporter activation with TLR2 alone (potentially pairing with endogenous TLR6) and to achieve maximal activation upon overexpression of exogenous TLR6. We also tested the impact of the previously described dominant negative (DN) TLR6 P680H variant. In this context, the level of $\mathrm{NF}-\kappa \mathrm{B}$ activation induced by this $\mathrm{DN}$ form was reduced of $70 \%$ with respect to the ancestral TLR6 and, importantly, it was also considerably lower than that observed with TLR2 alone. This suggests that, in our experimental setting, the transfected TLR6 construct is in large excess and overrides the contribution of the endogenous TLR6.

We now clarify this point in the revised version of the manuscript and provide details of our procedure (see page 13)

5- Is there a reason for the double band in figure 2c for the TLR1 I602S variant?; lower panel of all western blots: What was the loading control for these analyses?

As to the double band of the TLR1 I602S variant, the in silico analysis of the protein sequence predicts that the substitution of the isoleucine at position 602 with a serine residue creates a potential serine/threonine kinase phosphorylation motif. Thus, the slower migrating band of TLR1 I602S could correspond to the phosphorylated protein. This possibility is now presented in

John Wiley \& Sons, Inc. 
the manuscript (see page 12)

The reviewer is correct in that we omitted to mention that the loading control was $\beta$-actin. We apologize for this and have amended the figures accordingly.

6-The investigated populations are hardly described (how many individuals were investigated in the sub-Saharan population for instance?)

This comment was expressed by the other reviewers as well. We now describe the populations used in more detail in the revised Material and Methods section.

7-The functional characterisation is reduced to $\mathrm{Nf}-\mathrm{kB}$ reporter assays and FACS-analysis; the authors should include cytokine secretion of primary cells of mutation carriers and wild-type carriers as well.

Indeed, it would be extremely interesting to study the ex vivo effects of the functionally relevant variants by measuring cytokine secretion from primary donor cells. The difficulty is that most of these variants are singletons, i.e. present in a single heterozygote individual. It would be virtually impossible to recruit a sufficient number of healthy donors to test the ex vivo functional effects of these rare mutations. One exception is the TLR1 I602S variant, which is present at high frequency among Europeans ( $50 \%)$. For this variant, it has already been demonstrated that monocytes obtained from individuals who are homozygous for TLR1 602S secrete significantly lower levels of TNF- $\alpha$ in response to $\mathrm{Pam}_{3} \mathrm{CSK}_{4}$, when compared to monocytes from individuals who carry at least one TLR1 602I allele (Johnson et al. J Immunol 2007, cited in the discussion).

8- The Polyphen algorithm is neither mentioned in the abstract nor the introduction but it is interpreted. Is it necessary to mention Polyphen when having functional data? What are the consequences, what is the major result, the take home message of the comparison of functional TLR-data on the one hand and software prediction on the other? Why was Polyphen included? Is this manuscript an evaluation of Polyphen prediction? If not, my suggestion is to omit it.

The major aim of this study was to functionally characterize the variants identified. Because of the wide use of the Polyphen algorithm by the scientific community, we believe it is important to compare predicted and experimental functional data. However, as suggested by other reviewers, we have now used the recently released version of Polyphen (PolyPhen-2), which is considered a much better prediction algorithm (Adzhubei et al. 2010. Nature Methods). As suggested by this reviewer, we are now more clearly comparing and discussing the results obtained by Polyphen and our experimental data. Please, see page 18-19 in the Discussion section as well as the new Table 1 where we have added an extra column reporting the actual $\mathrm{NF}-\kappa \mathrm{B}$ activation data compared to the Polyphen predictions.

9. Table 1: the Polyphen data should be excluded and the results of the functional characterisation should be included here. How many alleles/chromosomes were investigated/population? Following this, figure 1 will not contain additional data and could be skipped.

With respect to Polyphen, see previous point. As requested, the results of the functional characterisation have been now included in Table 1. With respect to sequence variation for the TLR1-2-6 gene family, it was determined for a total of 158 individuals (316 chromosomes) representative of the populations of major geographical regions (63 fromSub-Saharan Africa,47 from Europe and 48 from East-Asia). This has now been clarified in the Materials and Methods section. 


\section{Referee: 2}

Comments to the Author

This is a very convincing study exploring the impact of naturally occurring mutation in the TLR1-2-6gene family. I have a couple of minor comments that the authors may want to consider in the revised version of the manuscript:

1. It is a bit unfair to the reader to refer to Barreiro et 2009 for details on the populations that have been used here. I would appreciate a table summarizing the most important features.

This criticism has been expressed by all reviewers. We now describe the populations used in much more detail in the Material and Methods section.

2. the authors used the polyphen algorithm to predict the functional impact of non synonymous mutations. recently a new version of this algorithm has been released (Polyphen 2) with, from my point of view, clearly improved prediction properties. Do they author used this last algorithm? if not I think this could be an interesting try.

The reviewer is correct in pointing out the release of the new version of the Polyphen algorithm, considered a better predictor than the previous version. We have used Polyphen v 2 and discuss these new data accordingly in the revised version of the manuscript.

3. How was chosen the $20 \%$ threshold to define 'functionally relevant' variant?

The threshold was indeed arbitrarily chosen. We preferred to focus on the variants that exhibited

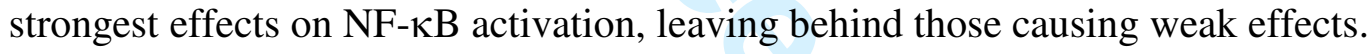

4. they mention 17 newly reported variant. Which database did they check to define a variant as new? In particular, did they used the 1000genomes project database? If not his could be also interesting.

We used dbSNP database to determine whether SNPs were new or not. As advised by the reviewer, we have now verified if these potentially new SNPs were present in 1000 genomes. Taking this into account, 14 SNPs remain novel in our paper.

5. there is no mention at all of the number of mutations per individual, i.e. did they observe individuals with two copy of NS mutations (either at the same position or not)?; on the same note is it correct to assume that these individuals did not present with any particular phenotype? if not this should be mention somewhere in the discussion.

Indeed, we found that some individuals were homozygous for a given NS mutation or had more than one NS mutation. We now provide the genotype information for each individual and each mutation as three Supplementary Tables (see Supp. Tables S1-S3)

As to the phenotype of the individuals, they are all considered as healthy donors. Obviously, we cannot exclude that individuals harboring more than one 'functionally relevant' mutation may be at higher risk for disease if exposed to particular pathogens. The healthy donor state of the individuals is now explicitly stated and clarified in the materials and methods. 
Referee: 3

Comments to the Author

Numerous studies have reported on the association between human Toll-like receptor polymorphisms and the incidence of disease. This paper by Meriem Ben-Ali and colleagues has systematically analyzed the function of naturally occurring nonsynonymous TLR variants in HEK cells using an NF-kB reporter system. The authors have also considered whether certain receptor variants exhibit altered cell surface expression. Finally, the ability of an algorithm (PolyPhen) to predict the impact of nonsynonymous variants on function was compared with the actual experimental results. Overall, the paper is straight forward, well written, statistically sound. Moreover, the paper provides a resource for labs studying TLR polymorphisms while attempting to interpret the results of genetic association studies. One caveat to the work is that all of the data are derived from in vitro systems which may not accurately reflect the effect of polymorphisms in vivo. Additionally, the authors only examined one, albeit central signaling output namely, $\mathrm{NF}-\mathrm{kB}$. These concerns merely reflect limitations of the experimental systems used and not the quality of work performed in this study. Addressable concerns are minor and as follows:

1) Table 1 should include a final column showing the actual results of the functional assays. This would allow the reader to directly compare the Polyphen results with that of the actual results for each individual polymorphism.

We thank the reviewer for this suggestion that will be helpful to the readers. We have now added an extra column in Table 1 , showing the actual results (\% of NF- $\kappa \mathrm{B}$ defect) for each of the 41 variants studied.

2) There is a vast literature on disease association with polymorphisms in TLRs 1, 2 and 6. The authors should take advantage (and perhaps reference) the excellent reviews that have been written on the subject such as: Garantziotis S, Hollingsworth JW, Zaas AK, Schwartz DA. The effect of toll-like receptors and toll-like receptor genetics in human disease. Annu Rev Med. 2008;59:343-59; Misch EA, Hawn TR. Toll-like receptor polymorphisms and susceptibility to human disease.Clin Sci (Lond). 2008 Mar;114(5):347-60; Tapping RI, Omueti KO, Johnson CM. Genetic polymorphisms within the human Toll-like receptor 2 subfamily. Biochem Soc Trans. 2007 Dec;35(Pt 6):1445-8.

We now discuss and cite these association studies in the revised version of the manuscript.

3) A systematic study of TLR diversity in humans was published last year and is certainly worthy of mention in the context of the work here: Georgel P, Macquin C, Bahram, S. The heterogeneous allelic repertoire of human toll-like receptor (TLR) genes. PLoS One 2009 Nov 17;4 (11): $e 7803$.

We now discuss and cite this study in the revised version of the manuscript.

4) The most critical paper that the authors overlooked was one in which an association analysis of more than 1,500 individuals revealed consistent associations between genetic variants in both TLR1 I602S with susceptibility to leprosy. The paper provides an excellent discussion of the expansion of the polymorphism in European populations: Won, SH, Gochhait S, Malhotra D, et al. Leprosy and the adaptation of toll-like receptor 1. PLoS Pathog. 2010 Jul 1;6:e1000979.

We now discuss and cite this study in the revised version of the manuscript. 
5) When discussing evolution and selection of polymorphisms within the family the authors should read and consider referencing the following paper: Enard D, Depaulis, F, Roest and Crollius H. Human and non- human primate genomes share hotspots of positive selection. PLoS Genet. 2010 Feb 5;6(2):e1000840.

We thank the reviewer for this suggestion. We now discuss and cite this study in the revised version of the manuscript.

John Wiley ${ }^{6}$ Sons, Inc. 


\section{EDITORIAL BOARD'S COMMENTS}

All three referees gave a good evaluation of this work. There are, however, a rather large number of points that will require careful consideration and correction, in particular questions raised by referee1 on the use of $\mathrm{HEK} 293$ cells, the possibility of inducing apoptosis by receptor overexpression, etc. Please carefully answer these concerns. Please make edits to the manuscript using "track changes" if possible, or by highlighting added text.

First of all, we thank the Editorial Board as well as the three Referees for the useful suggestions, which we believe have improved the clarity and the quality of the manuscript. As you can see in the individual responses to the Referees (uploaded separately in the cover letter since there is a figure), we have now addressed all comments and concerns (paying special attention to the questions of the use of HEK293 cells, the possibility of inducing apoptosis by receptor overexpression, etc., see responses to referee 1). In addition, although Referee 1 suggested to leave out the PolyPhen predictions, Referees 2 and 3 appeared more interested in these information and thus we have kept this part. As requested by one Referee, we have re-analysed the data using the PolyPhen-2 version and, as suggested by Reviewer 1, we are more explicitly discussing and comparing the results of this predictive method with the actual experimental data.

Please, find here enclosed the revised version of the manuscript with the corresponding changes, which are now highlighted in red.

We hope that we have now satisfied both the reviewers' and the editorial board's comments and that the manuscript is suitable for publication in Human Mutation. 


\section{MANAGING EDITOR COMMENTS:}

Please respond to these points under "Response to Managing Editor", otherwise the final decision could be delayed.

1) Please include the OMIM accession number for the genes discussed, at first mention in the Introduction.

--Human gene symbols must be in all caps-italics. Protein symbols should be all caps-Roman. --Please ensure that you use HUGO HGNC-approved gene symbols. Common gene symbol aliases may also be used at first mention. OMIM entries do not always feature the approved symbol prominently. Verify gene symbols at http://www.genenames.org/

Done

2) The mutation nomenclature must follow the format indicated in the Author Instructions (see the website http://www.hgvs.org/mutnomen/ and also the nomenclature checklist at http://www.hgvs.org/mutnomen/checklist.html ). Things to watch for:

a-Mention the GenBank reference sequence and version number for the genes studied (i.e., include the decimal point following the accession number in the sequence record) in the Materials and Methods and as a footnote to the relevant table.

Done

b-Clearly indicate in the Methods text and tables that the DNA mutation numbering system you use is based on cDNA sequence. Explanatory text is required in legends and text, for example:

"Nucleotide numbering reflects $c D N A$ numbering with +1 corresponding to the $A$ of the ATG translation initiation codon in the reference sequence, according to journal guidelines (www.hgvs.org/mutnomen). The initiation codon is codon 1."

Done

c-Authors are advised to check sequence variant descriptions using the Mutalyzer program (http://www.LOVD.nl/mutalyzer/) - using batch mode, all variants for a gene can be analyzed at once.

Done

d-Please verify that the mutations reported (especially novel ones) have been or will be submitted to an existing locus-specific database for the genes involved. Visit the HGVS-LSDB list to search for databases: http://www.hgvs.org/dblist/glsdb.html

Novel SNPs have been now submitted to the dbSNP database, and we are waiting for the corresponding \#rs numbers.

3) On resubmission:

a-Please double check the author names and affiliations carefully. These are often a source 
of typographical errors.

Done

b-Title Page, Abstract (180-200 words max), Key Words, Main Text, References, and

Figure Legends should be combined into one file for the manuscript and submitted as a *.doc file.

Done

c-Figures for main article must be submitted as separate files with high resolution (at least 200 dpi) as *.tif or *.eps format only.

--For color figures in print: submit two files for each color figure: one in CMYK color space and one in RGB color space (with the true color you wish to have published). If you cannot provide CMYK color e-files for the print version, please let me know. (see below regarding color costs)

--For online-only color figures, please only submit one RGB color space file.

Only Figure 1 should be in color in print, and is submitted in two separate files (CMYK and RGB). The other figures are online-only figures.

d-Tables must be submitted individually as separate*.doc files (with their titles and legends included). Please use the MS Word table format if possible. Excel (*.xls) files should not be submitted.

Table 1 is provided as a separate *.doc file, with its title and legend.

e-Any Supporting Tables or Figures should be named and cited from the text as follows: 'Supp. Table S1' and 'Supp. Figure S1' (see below).

--If possible, do not use custom paper sizes - Letter or A4 are preferred.

Done

f-Supporting Figures and Tables should be prepared in a single MS Word *.doc file labeled 'Supp_Mat', with Figures preceding Tables. Each table/figure should be accompanied with its legend.

The three supporting Tables S1-S3 have been merged into a single *.doc file

4) Please check to see that the references follow the journal's standard format and are cited properly. See our online Author Instructions

http://onlinelibrary.wiley.com/journal/10.1002/\%28ISSN\%291098-

1004/homepage/ForAuthors.html

Done

5) Figure 1 requires color in the print version in order to be fully understood, because the John Wiley $\stackrel{9}{\&}$ Sons, Inc. 
colors "blue" and "orange" are required in order to see which mutations are being referred to. As noted in our Author Instructions, there are no page charges for publication in Human Mutation but there are costs associated with publication of color images in print: \$500 USD per printed color page. Alternatively, there is an option of publishing the color images in black-and-white in the print article and in color online, at no cost to you - but no information must be lost in a conversion to $\mathrm{b} / \mathrm{w}$ from color for the print version. For example, if the "blue" text in the figure was also put in italic and the "orange" text was also placed in bold type, and that information was added to the legend ("black, blue/italic, and orange/bold"), then the figure could be interpreted in $b / w$. Please confirm your preference in reply. (Non-essential color images can be published in color online only at no cost. The other color figures appear to not require color in print, but color online will be attractive.)

Figure 1 should be in color in print, and is submitted in two separate files (CMYK and RGB). The other figures are online-only figures.

6) Human Mutation can accommodate researchers funded by agencies requiring open access publication. More information on Wiley-Blackwell's policy is available at: http://olabout.wiley.com/WileyCDA/Section/id-406241.html

7) IMPORTANT INFORMATION REGARDING PREPRINTS a-Human Mutation is now publishing online preprints of accepted manuscripts prior to typesetting and page proof corrections. It is therefore crucial that you revise your manuscript carefully so that errors (typographical and grammatical) are corrected BEFORE the final accepted manuscript is posted online. The accepted preprint version will remain online until the corrected proofs are received and the typeset manuscript is finalized. At that time, the preprint version will be replaced with the final, typeset version online, in Early View.

b-It is essential that you submit a copyright transfer agreement (CTA) upon submission of your revised manuscript. This will avoid delay in publication of your article upon

acceptance. If possible, the CTA must be signed by the corresponding author and should be signed by all contributing authors if practical. All authors must be made aware of the CTA and the rights it conveys to them.

The CTA can be found here: www.wiley.com/go/ctaaus and must be filled out completely, including the article title and manuscript number. Please fax it to this number: (201) 748-6091.

The CTA has now been faxed. 


\title{
Functional characterisation of naturally occurring genetic variants in the human TLR1-2-6 gene family
}

\author{
Meriem Ben-Ali ${ }^{1,2}$, Beatrice Corre ${ }^{3,4}$, Jérémy Manry ${ }^{1,2}$, Luis B. Barreiro ${ }^{5}$, Hélène Quach ${ }^{1,2}$, \\ Michele Boniotto $^{1,2}$, Sandra Pellegrini ${ }^{3,4}$, Lluís Quintana-Murci ${ }^{1,2, *}$ \\ ${ }^{1}$ Institut Pasteur, Human Evolutionary Genetics, Department of Genomes and Genetics, F- \\ 75015 Paris, France \\ ${ }^{2}$ Centre National de la Recherche Scientifique, URA 3012, F-75015 Paris, France \\ ${ }^{3}$ Institut Pasteur, Unit of Cytokine Signaling, Department of Immunology, F-75015 Paris, \\ France \\ ${ }^{4}$ Centre National de la Recherche Scientifique, URA 1961, F-75015 Paris, France \\ ${ }^{5}$ Department of Human Genetics, University of Chicago, Chicago, U.S.A.
}

\begin{abstract}
*To whom correspondence should be addressed at: Institut Pasteur, Human Evolutionary Genetics, Department of Genomes and Genetics, F-75015 Paris, France. Phone: +33-1-40 61 34 43. Fax: +33-1-45 6886 39. e-mail: quintana@ pasteur.fr
\end{abstract}

John Wiley ${ }^{-1-}$ - Sons, Inc. 


\begin{abstract}
Toll-like receptors are considered an essential component of the innate immune system, initiating inflammatory responses following infection of the host. Humans have 10 functional TLRs, differing in their subcellular distributions and the microbial agonists they sense. The phylogenetically-conserved TLR1-2-6 family is unique in that TLR1 and TLR6 form heterodimers with TLR2 to mediate signalling in response to agonists. Epidemiological genetic studies have identified several TLR variants that appear to influence susceptibility to infectious diseases, but the functional consequences of which remain largely unknown. Here, we assessed the functional impact of the TLR1-2-6 variants with altered amino-acid sequences segregating naturally in the human population. We used an $\mathrm{NF}-\kappa \mathrm{B}$ reporter assay in TLR-transfected human embryonic kidney $293 \mathrm{~T}$ cells stimulated with the corresponding TLR agonists. We found that among the 41 naturally occurring variants with amino-acid alterations identified in the TLR1-2-6 family, 14 of them (5 TLR1, 4 TLR2, and 5 TLR6 variants) displayed marked impairment of NF- $\kappa \mathrm{B}$ activation. Most of these variants are present at very low population frequencies and are population-specific. These observations suggest that rare, non-synonymous TLR mutations are likely to have deleterious effects on immune responses and may therefore contribute to complex susceptibility to infection at the population level.
\end{abstract}

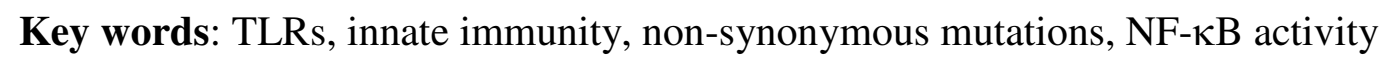




\section{INTRODUCTION}

The innate immune system constitutes the first line of host defence against infection and plays a crucial role in the immediate recognition of invading pathogens, subsequently triggering proinflammatory responses [Janeway and Medzhitov, 2002; Lemaitre and Hoffmann, 2007; Medzhitov and Janeway, 2000; Medzhitov and Janeway, 1997]. This early response involves the recognition of evolutionarily conserved structures on pathogens — pathogen-associated molecular patterns (PAMPs) — through a limited number of germline-encoded pattern recognition receptors (PRRs) [Akira et al., 2006; Beutler et al., 2003; Janeway and Medzhitov, 2002; Kimbrell and Beutler, 2001; Medzhitov and Janeway, 1997]. The Toll-like receptors (TLRs) are the PRRs most extensively studied from clinical, epidemiological and evolutionary angles [Akira and Takeda, 2004; Casanova et al., 2011; Kawai and Akira, 2007; Medzhitov, 2001]. TLRs are type-I transmembrane proteins belonging to the interleukin-1 receptor family. They possess an N-terminal leucine-rich repeat (LRR) ligand-binding domain, a transmembrane domain and a C-terminal TIR domain for intracellular signalling. TLR signalling is initiated by agonist-induced dimerisation, which triggers intracellular signals ultimately culminating in the production of proinflammatory cytokines and interferons (IFNs), which orchestrate adaptive immune responses [Akira and Takeda, 2004; Kawai and Akira, 2007; Medzhitov, 2001; West et al., 2006]. Humans have ten different TLRs, which differ in terms of the PAMPs they sense, subcellular distribution and the nature of their responses [Akira and Takeda, 2004; Akira et al., 2006; Beutler, 2004; Kawai and Akira, 2006; Kawai and Akira, 2007; Leulier and Lemaitre, 2008; Medzhitov, 2001; West et al., 2006]. TLR3 (MIM\#603029), TLR7 (MIM\#300365), TLR8 (MIM\#300366) and TLR9 (MIM\#605474) are typically located in intracellular compartments, such as the endosomes, whereas TLR1 (MIM\#601194), TLR2 (MIM\#603028), TLR4 (MIM\#603030), TLR5 (MIM\#603031) and TLR6 (MIM\#605403) are generally expressed on the cell surface. 
Intracellular TLRs sense nucleic acid agonists and are involved principally in the recognition of viruses, whereas cell surface-expressed TLRs detect various other products, mostly of bacterial, parasite and fungal origin.

Evolutionary analyses of vertebrate TLRs have revealed various phylogenetically conserved TLR families, each of which recognises a general class of PAMPs [Beutler and Rehli, 2002; Leulier and Lemaitre, 2008; Roach et al., 2005]. The TLR family specific for lipopeptide PAMPs includes TLR1, TLR2 and TLR6. Human TLR10 (MIM\#606270) also belongs to this family, but its agonists and specific functions remain unknown. This family, which is known under various names but, for convenience, will be referred to here as the “TLR1-2-6 gene family", is unique in that TLR1 and TLR6 must co-operate with TLR2, forming TLR2/TLR1 or TLR2/TLR6 heterodimers, to mediate signalling in response to microbial agonists [Ozinsky et al., 2000]. The TLR2/1 heterodimer recognizes various lipoproteins, including those from mycobacteria and meningococci [Takeuchi et al., 2002], whereas the TLR2/6 heterodimer senses peptidoglycan and mycoplasma lipoproteins [Takeuchi et al., 2001].

Due to the central role of TLRs in the innate immune system, genetic variation in this gene family may affect protein function and consequently alter susceptibility to infections. Several epidemiological genetics studies have investigated the contribution of variation in the genes encoding TLR1, 2 and 6, and TLRs in general, to susceptibility to infectious diseases (for comprehensive reviews, see [Casanova et al., 2011; Garantziotis et al., 2008]). For example, the TLR1 p.Ile602Ser variant, which affects the trafficking of the receptor to the cell surface, has been associated with protection against leprosy and leprosy reversal reaction [Johnson et al., 2007; Misch et al., 2008; Wong et al., 2010]. Genetic variation in TLR2 has been reported to alter the susceptibility to various inflammatory and infectious diseases. Specifically, the well known TLR2 p.Arg753Gln variant appears to be associated with 
increased susceptibility to tuberculosis and acute rheumatic fever in children [Berdeli et al., 2005; Ogus et al., 2004]. Similarly, the TLR6 p.Ser249Pro variant has been associated with a decreased risk of asthma in African-Americans [Tantisira et al., 2004]. More generally, nonsynonymous polymorphisms in the TLR6-TLR1-TLR10 gene cluster have been found to be significantly associated with tuberculosis disease in certain ethnic groups [Ma et al., 2007]. However, the clear involvement of TLR variants in the complex genetic control of infectious pathogenesis has yet to be replicated in most cases and the functional consequences of $T L R$ mutations remain mostly unknown [Casanova et al., 2011].

In this study, we investigated the functional impact of naturally occurring nonsynonymous variations in the TLR1-2-6 gene family. We used an NF- $\mathrm{B}$ gene reporter assay system for the functional testing of 40 non-synonymous variants and one stop mutation, all corresponding to amino acid-altering mutations identified in a previous comprehensive sequence-based population study [Barreiro et al., 2009]. Fourteen of these variants presented substantial functional impairment. Furthermore, most of these functionally relevant mutations were observed at low population frequencies $(<5 \%)$, suggesting that low-frequency nonsynonymous mutations probably have deleterious effects on host immunity to infection. 


\section{MATERIALS AND METHODS}

\section{Population samples}

Sequence variation for the TLRI-2-6 gene family was determined for a total of 158 individuals (316 chromosomes) representative of the populations of major geographical regions (Sub-Saharan Africa, Europe and East-Asia) (see [Barreiro et al., 2009]). The description of the specific population samples can be found in ALFRED (http://alfred.med.yale.edu) using the unique IDs given in parentheses. Sub-Saharan Africans were represented by Yorubans from Nigeria (31 individuals from UID “SA000036J”) and Chaggas from Tanzania (32 individuals from UID “SA000487T”); Europeans were represented by Danes (23 individuals from UID "SA000046K”) and Chuvash from Russia (24 individuals from UID “SA000491O”), and East-Asians were represented by Han Chinese (24 individuals from UID “SA000009J") and Japanese (24 individuals from UID "SA000010B”). All individuals were healthy donors from whom informed consent was obtained. This study was approved by the Institut Pasteur Institutional Review Board (no. RBM 2008.06).

\section{Mutation detection}

All of the exon regions of TLRI (accession no. NM_003263.3), TLR2 (accession no. NM_003264.3) and TLR6 (accession no. NM_006068.3) were sequenced (for details see [Barreiro et al., 2009]). Sequences were obtained with the Big Dye terminator kit and an Applied Biosystems 3730 automated sequencer. Sequence files and chromatograms were inspected with GENALYS software [Takahashi et al., 2003]. For quality control, and to prevent allele-specific amplification, we designed new primers and repeated sequence reactions whenever new mutations were identified in primer-binding regions. All singletons or ambiguous polymorphisms were systematically reamplified and resequenced. The gDNA nucleotides and the amino acids of the protein were numbered based on GenBank sequence 
information. The mutation nomenclature follows the journal's guidelines

(www.hgvs.org/mutnomen) according to the reference sequence with +1 as the A of the ATG initiation codon.

\section{Prediction of the functional impact of non-synonymous mutations}

The functional impact of all amino acid-altering mutations (i.e. benign, possibly damaging or probably damaging) was predicted with the PolyPhen-2 algorithm [Adzhubei et al., 2010]. This method, which considers protein structure and/or sequence conservation information for each gene, has been shown to be the best predictor of the fitness effects of amino-acid substitutions. The domains of the various TLR proteins were identified with TollML: a database of Toll-like receptor structural motifs [Gong et al., 2010].

\section{Plasmid constructs and site-directed mutagenesis}

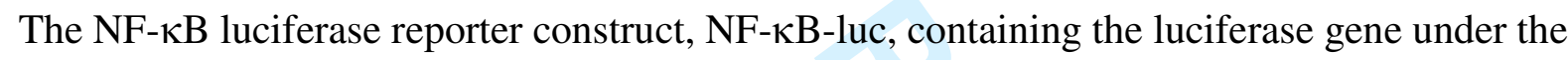
control of six $\kappa \mathrm{B}$ sites from the thymidine kinase promoter was kindly provided by Oreste Acuto. The Renilla luciferase construct was purchased from Promega (Promega, Madison, WI). All TLR constructs were purchased from Invivogen (InvivoGen, San Diego, CA). Allelic variants of TLR1, TLR2 and TLR6 were generated with the QuikChange site-directed mutagenesis system, according to the manufacturer's instructions (Stratagene, La Jolla, CA). All constructs were systematically verified by sequencing the complete TLR gene with an Applied Biosystems 3730 automated sequencer (Applied Biosystems, Foster City, CA).

\section{Cell culture, transfection and dual luciferase reporter assay}

HEK 293T cells were cultured in DMEM supplemented with 10\% FCS, 100 IU penicillin and $100 \mu \mathrm{g} / \mathrm{ml}$ streptomycin (Invitrogen, Carlsbad, CA), at $37^{\circ} \mathrm{C}$, in a humidified incubator, under 
an atmosphere containing $5 \% \mathrm{CO}_{2}$. Cells were used to seed 24 -well plates $\left(5 \times 10^{4}\right.$ cells/well). The next day, cells (30 to 50\% confluence) were transiently transfected with the pNF-кB-luc, along with constructs encoding the various TLRs, in the presence of FuGene 6 reagent (Roche Diagnostics), according to the manufacturer's recommendations. All plasmids used for transfection were purified with the Endofree plasmid kit (Qiagen, Chatworth, CA). Briefly, cells were cotransfected with $100 \mathrm{ng}$ of NF- $\mathrm{KB}$ reporter construct, TLR constructs and $5 \mathrm{ng}$ of pRL-TK-Renilla luciferase construct (Promega, Madison, WI) as a control for transfection efficiency. For each transfection, total DNA was adjusted to 300 or 400 ng with empty pcDNA3.1 vector. For TLR2/1 and TLR2/6 studies, cells were cotransfected with 2.5 or $5 \mathrm{ng}$ of TLR2 and $100 \mathrm{ng}$ of TLR 1 or $200 \mathrm{ng}$ of TLR6. For TLR2 experiments, $300 \mathrm{ng}$ of TLR2 variants was used for transfection. After 24 h, TLR2/1- and TLR2/6-transfected cells were stimulated for $4 \mathrm{~h}$ with $5 \mathrm{ng} / \mathrm{ml} \mathrm{Pam}_{3} \mathrm{CSK}_{4}$ and for $3 \mathrm{~h}$ with $10 \mathrm{ng} / \mathrm{ml} \mathrm{Pam}_{2} \mathrm{CSK}_{4}$, respectively, and TLR2-transfected cells were stimulated for $16 \mathrm{~h}$ with $10 \mathrm{ng} / \mathrm{ml} \mathrm{Pam}_{3} \mathrm{CSK}_{4}(\mathrm{EMC}$ microcollections). Supernatants were discarded and cells were lysed in $100 \mu$ l of passive lysis buffer (Promega, Madison, WI) and used for dual luciferase assays (firefly and Renilla luciferase activities) carried out according to the manufacturer's instructions. Luciferase activity was normalised by dividing by Renilla luciferase activity, to correct for transfection efficiency, and the results are expressed as the mean relative stimulation $( \pm$ SD) in three independent experiments, each performed in triplicate.

\section{Flow cytometry analysis}

HEK293T cells were transfected in six-well plates with HA-tagged TLR1, TLR2 or TLR6 expression vector or empty vector alone ( $2 \mu \mathrm{g}$ each). Transfections were performed with the FuGENE 6 transfection reagent (Roche), at a 3:1 ratio of FuGENE 6 to DNA. The medium was replaced $24 \mathrm{~h}$ after transfection, and cells were removed from the plate into chilled PBS 
(pH 7.4) $48 \mathrm{~h}$ after transfection and incubated with the corresponding Abs. Cell-surface expression of TLR1 and TLR2 was measured with the mAbs GD2.F4 and T2.5, respectively (eBioscience). Appropriate isotype-matched Abs were used as controls.

\section{Protein analysis}

For immunoblots, cells transfected with TLR variants were lysed in $100 \mu$ l of passive lysis buffer (Promega, Madison, WI) and centrifuged. Equal volumes of cell lysate were subjected to $10 \%$ SDS-PAGE under reducing conditions, and the resulting bands were transferred to a membrane and probed with an anti-HA tag antibody (Invivogen, San Diego, CA), followed by horseradish peroxidase-conjugated rabbit anti mouse IgG (JacKson ImmunoResearch, West Grove, PA). The signal was detected by incubation with Pierce ECL western blotting reagent (Thermo Scientific, Rockford, IL). 


\section{RESULTS}

Our re-sequencing effort of the three members of the TLRI-2-6 gene family in a panel of 158 healthy individuals originating from sub-Saharan Africa, Europe and East Asia allowed us to identify 40 non synonymous variants and one stop mutation that naturally segregate in the human population (Table 1, Supp. Tables S1-S3) [Barreiro et al., 2009]. The location of each variant within the protein (e.g. extracellular, transmembrane, TIR domains) is shown in Figure 1. When comparing these variants to those that are described in public databases, we found that 14 of them are newly identified mutations. We first used the PolyPhen-2 algorithm [Adzhubei et al., 2010] to predict the functional impact of all amino-acid altering variants described in this study. Interestingly, the vast majority of the variants predicted to be possibly or probably damaging $(\sim 80 \%)$ were observed at low frequencies $(<5 \%)$ and most of them were population specific.

In order to obtain unbiased results, we systematically characterized the 41 amino acid altering variants regardless of their population frequencies and their effect on protein structure and function as predicted by the PolyPhen algorithm [Adzhubei et al., 2010]. Each variant was generated by site-directed mutagenesis from the reference $T L R 1, T L R 2$ or TLR6 template corresponding to the ancestral form in the human population, defined on the basis of the chimpanzee sequence. We then assessed the ability of these constructs to activate NF- $\kappa$ B signalling, the major TLR-induced pathway, in transiently transfected human embryonic kidney (HEK) 293T cells stimulated with the most commonly used TLR-specific agonists. The variants found to be functionally relevant (i.e. TLR variants with levels of NF- $\kappa B$ activation more than $20 \%$ lower than the ancestral form) were further studied by measuring their cell surface expression by flow cytometry. 


\section{Functional characterisation of TLR1 variants}

Since TLR1 signals as a heterodimer with TLR2, the various TLR1 constructs were transfected into HEK293T cells together with the wild-type (ancestral) TLR2, and cells were stimulated with the $\mathrm{PAM}_{3} \mathrm{CSK}_{4}$ agonist. One of the 17 identified TLR1 non-synonymous variants (p.Ser248Asn) displayed a small loss of activity $(<20 \%)$, whereas five others (p.Pro315Leu, p.Tyr554Cys, p.Ile602Ser, p.Val651Ala and p.His720Pro) displayed severe impairment of activity with respect to the ancestral form (Fig. 2A). The p.Pro315Leu and p.Ile602Ser variants displayed the greatest loss of activity ( $70 \%$ and $60 \%$, respectively), with levels of NF- $\kappa$ B activation similar to those obtained with TLR2 alone. The other three variants (p.His720Pro and the newly reported p.Tyr554Cys, p.Val651Ala variants) displayed losses of NF- $\kappa \mathrm{B}$ activation of $\sim 30 \%, 50 \%$ and $40 \%$, respectively. In addition, two TLR1 variants (p.Ile75Thr and p.Ile460Val) were associated with levels of NF- $\kappa$ B activation $~ 25 \%$ and $12 \%$ higher, respectively, than for the ancestral form. The remaining 10 variants induced $\mathrm{NF}-\kappa \mathrm{B}$ activation to a similar extent to the ancestral form.

We further characterised the five functionally relevant TLR1 variants (p.Pro315Leu, p.Tyr554Cys, p.Ile602Ser, p.Val651Ala and p.His720Pro), by measuring their expression at the surface of transfected cells by flow cytometry with a widely used commercial anti-TLR1 mAb (GD2.F4) (Fig. 2B). No signal was detected on cells transfected with an empty vector, demonstrating an absence of endogenous TLR1 expression in HEK293T cells (data not shown). The p.Pro315Leu variant was not detected at the cell surface because, as reported in a previous study [Omueti et al., 2007], it is not recognized by the anti-TLR1 mAb.

Additionally, our flow cytometry analysis did not detect neither the p.Ile602Ser variant, whose intracellular trafficking is impaired [Johnson et al., 2007], nor the newly reported p.Tyr554Cys variant. By contrast, the p.Val651Ala and p.His720Pro variants were well expressed on the surface of transfected cells. Western blots of total cell lysates were 
performed to evaluate the overall expression levels of these variants. All variants were expressed to similar levels (Fig. 2C). The TLR1 p.Ile602Ser variant gave rise to a double band. The altered migration may be due to the presence of a novel site of phosphorylation.

\section{Functional characterisation of TLR2 variants}

We then assessed the functional consequences of changes in the amino-acid sequence of TLR2 (6 non-synonymous variants and one stop mutation), by measuring the response of transfected cells to the agonist $\mathrm{PAM}_{3} \mathrm{CSK}_{4}$. As expected, cells transfected with the R447Stop construct, which contains a premature stop codon in the extracellular portion of the receptor resulting in a truncated protein, failed to respond to stimulation (Fig. 3A). Among the six non-

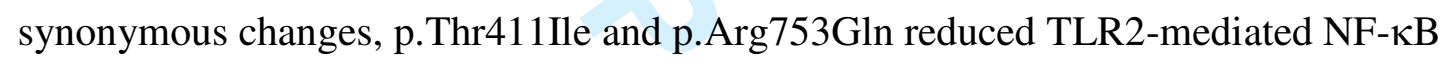
activation by about 50 and $75 \%$, respectively, whereas p.Pro631His reduced this activation by $\sim 30 \%$. The remaining three variants (i.e., p.Arg571His and the newly identified p.Asn89Asp and p.Ser636Arg) were functionally similar to the ancestral form (Fig. 3A).

Flow cytometry analysis revealed that, with the exception of the p.Arg447X stop variant whose expression was abrogated, all TLR2 variants were expressed at the surface of transfected cells (Fig. 3B). Western blot analysis showed that the four functionally relevant HA-tagged TLR2 variants were expressed at similar levels (Fig. 3C).

\section{Functional characterisation of TLR6 variants}

Since TLR6 needs to heterodimerize with TLR2 for signalling, the different TLR6 variants (i.e. 17 non-synonymous variants) were co-transfected with TLR2 and cells were tested for their response to the synthetic lipopeptide $\mathrm{PAM}_{2} \mathrm{CSK}_{4}$ agonist. Background levels of reporter activation were observed in cells transfected with TLR2 alone (Fig. 4A), probably due to the expression of "endogenous" TLR6 in the recipient cells. The amounts of the TLR6 and TLR2 
constructs used for transfection were optimised so as to reduce reporter activation with TLR2 alone (potentially pairing with endogenous TLR6) and to achieve maximal activation upon overexpression of exogenous TLR6 (Fig. 4A). As a control, we tested the impact on NF-кB activation of the previously described dominant negative (DN) TLR6 p.Pro680His variant [Okusawa et al., 2004]. We observed that the level of NF- $\kappa$ B activation induced by this TLR6 DN form was reduced of $\sim 70 \%$ with respect to the ancestral TLR6 and, importantly, it was also considerably lower than that observed with TLR2 alone (Fig. 4A). This suggests that, in our experimental setting, the transfected TLR6 construct is in large excess and overrides the contribution of the endogenous TLR6. Under these conditions, cells transfected with p.Ala210Thr, p.Ala210Gly or p.Arg247Lys showed a slight attenuation ( $<20 \%$ decrease) in their ability to respond to the $\mathrm{PAM}_{2} \mathrm{CSK}_{4}$ agonist, with respect to cells transfected with the ancestral TLR6. Cells transfected with p.Leu128Val, p.Leu194Pro, p.Ala474Val, p.Asn690Thr or p.Gln708His constructs displayed a stronger decrease in NF- $\kappa \mathrm{B}$ activation (Fig. 4A). p.Leu128Val showed the most severe impairment, with a loss of function of about $40 \%$ with respect to the ancestral form. Indeed, the activity displayed by p.Leu128Val was similar to the background activity observed with TLR2 alone. All the remaining variants displayed levels of NF- $\kappa \mathrm{B}$ activation similar to those of the ancestral form.

Western blots of HA-tagged TLR6 and its variants showed that all functionally relevant variants were expressed to similar extents (Fig. 4B). The cell surface levels of the TLR6 variants could not be adequately assessed by FACS, given the presence of endogenous TLR6 (data not shown).

\section{PolyPhen predictions and functional characterisation}

The use of computational methods to predict the functional impact of protein sequence variants is a useful tool in medical and evolutionary genetics. The PolyPhen algorithm 
predicts the impact of nonsynonymous variants (benign, possibly damaging, or probably damaging) on the structure and function of the protein, using alignments of orthologous gene sequences from multiple species and, when available, physical considerations, such as the three-dimensional structures of proteins [Adzhubei et al., 2010]. We compared the PolyPhen predictions for each of the 40 non-synonymous mutations tested in this study (Table 1) with their actual, observed effect on the parameter considered here: NF- $\kappa \mathrm{B}$ activation. The vast majority of mutations predicted to be benign did not substantially affect NF- $\kappa \mathrm{B}$ activation. In contrast, most variants predicted to be possibly or probably damaging showed indeed a substantial decrease in NF- $\kappa$ B activation (Fig. 5A). Specifically, among the eleven nonsynonymous SNPs that were predicted to be probably or possibly damaging by PolyPhen, our

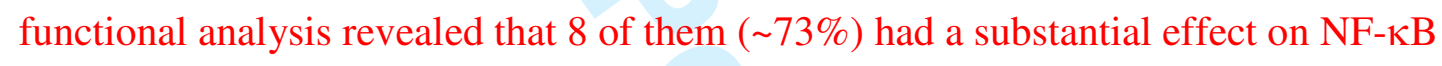
activation (i.e., more than a $20 \%$ decrease in NF- $\kappa$ B activation with respect to the ancestral form). Conversely, only five of the 29 SNPs predicted to be "benign" ( 17\%) reduced substantially NF- $\kappa \mathrm{B}$ activation. Interestingly, mutations located the TIR domain tended to have a more severe impact on signalling than those affecting the extracellular domain (Fig. 5B). All TIR domain variants were maintained at low frequency $(<5 \%)$ in each of the three populations studied, suggesting that variation affecting the amino-acid sequence of this domain is under strong evolutionary constraints. 


\section{DISCUSSION}

Assessment of the extent to which amino acid sequence-altering variation segregating in the general population affects protein function constitutes a step towards a clearer understanding of the way in which host genetic variants contribute to complex disease susceptibility and/or progression in humans. We used the TLR1-2-6 gene family as a model to investigate the functional consequences of 41 naturally occurring variants with altered amino-acid sequences previously identified in an unbiased population genetics resequencing scheme in a multiethnic panel of healthy individuals [Barreiro et al., 2009].

We found that five of the 17 non-synonymous TLR1 variants displayed strong impairment of NF- $\mathrm{KB}$ activation (Fig. 2A). Two of these strongly impaired variants (p.Pro315Leu and p.Ile602Ser) were previously shown to impair TLR1/2 function [Barreiro et al., 2009; Hawn et al., 2007; Johnson et al., 2007; Omueti et al., 2007]. The p.Pro315Leu amino-acid change, in the outer loop of LRR11, drastically reduced NF- $\mathrm{B}$ activation in response to $\mathrm{PAM}_{3} \mathrm{CSK}_{4}$, consistent with previous observations showing that p.Pro315Leu affects cellular responses to various microbe-derived agonists [Omueti et al., 2007]. The p.Ile602Ser variant, which was also associated with a major loss of NF- $\kappa \mathrm{B}$ activation, is known to affect receptor trafficking to the plasma membrane, impairing responses in both primary monocytes and heterologous expression systems [Barreiro et al., 2009; Hawn et al., 2007; Johnson et al., 2007]. With respect to the three remaining functionally relevant TLR1 variants, (p.Tyr554Cys, p.Val651Ala and p.His720Pro), p.Tyr554Cys exhibits a substantial activity loss and is not detected on the surface of transfected cells (Fig. 2B). The tyrosine 554 is located within the C-terminal LRR domain and its substitution with cysteine residue, may affect the conformation of the ectodomain. Finally, p.Val651Ala and p.His720Pro were well expressed on the surface of transfected cells, suggesting correct folding and trafficking to the plasma membrane (Fig. 2B). However, the location of these two 
amino-acid substitutions in the TIR-domain suggests a possible impact on interaction with intracellular partners, such as the adaptor MyD88.

The case of the TLR1 p.Ile602Ser variant, which strongly impacts protein function, merits further discussion. This mutation characterises a haplotype, encompassing the TLR10TLR1-TLR6 gene cluster, which is present at high frequency in Europeans (up to 30\%), while it is rare in Africans and Asians. Interestingly, this haplotype has been shown to be under strong positive selection in the European population [Barreiro et al., 2009; Pickrell et al., 2009]. Given both the signature of positive selection detected and the functional consequences of the TLR1 p.Ile602Ser variant, it is highly plausible that the attenuation of TLR1-mediated signaling, leading to a weaker inflammatory response, has conferred a selective advantage in Europeans. The association between the hyporesponsiveness p.Ile602Ser variant and protection against leprosy pathogenesis suggests that mycobacteria, or other pathogens that are recognized by TLR1, may be the agents underlying the patterns of selection and, as a consequence, may have contributed to the present global distribution of the p.Ile602Ser variant [Johnson et al., 2007; Misch et al., 2008; Wong et al., 2010]. Regardless of the genuine agent of selection, the TLR1 p.Ile602Ser variant falls in a genomic region that has been recently found to be a hotspot of positive selection in human and non-human primates [Enard et al., 2010]. The concomitant detection of positive selection targeting the TLR10TLR1-TLR6 gene cluster in human, chimpanzee and orangutan [Barreiro et al., 2009; Enard et al., 2010; Pickrell et al., 2009] strongly support the notion that this genomic region has played a major adaptive role in host resistance to a broad spectrum of bacteria in multiple species.

With respect to TLR2 naturally-occurring amino-acid changes, three of them (p.Thr411Ile, p.Pro631His and p.Arg753Gln) displayed impaired activity and one (p.Arg447X) was non-responsive (Fig. 3A). This latter is truncated but still able to interact with TLR1, TLR6 and/or TLR2 via the extracellular domain, thereby forming non-functional 
heterodimers or homodimers, respectively [Merx et al., 2007]. p.Arg753Gln, which greatly reduced NF-KB activation, consistent with previous reports [Kormann et al., 2009; Merx et al., 2007], is located in the TIR-domain, suggesting possible effects on its interaction with MyD88. The prevalence of the p.Arg753Gln variant has been examined in several disease contexts and this variant has been associated with an increase in susceptibility to tuberculosis, acute rheumatic fever in children, and CMV disease after liver transplantation [Berdeli et al., 2005; Kijpittayarit et al., 2007; Ogus et al., 2004]. With respect to the p.Thr411Ile variant, in contrast with a recent study that observed no decrease in NF- $\kappa \mathrm{B}$ activity in response to $\mathrm{PAM}_{3} \mathrm{CSK}_{4}$ [Kormann et al., 2009], our data showed that this variant drastically reduced NF$\kappa \mathrm{B}$ activation in agreement with early observations [Merx et al., 2007]. The location of the p.Thr411Ile variant in the extracellular domain suggests an impact on the ligand-binding properties of TLR2. Discrepancies between studies are also patent for the p.Pro631His variant. While two previous studies reported that the NF- $\kappa \mathrm{B}$ pathway is not altered by p.Pro631His [Kormann et al., 2009; Merx et al., 2007], we observed that this variant does affect NF- $\kappa \mathrm{B}$ activation. In support of our observation, p.Pro631His has been shown to have a dominant negative effect on wild-type TLR2 signalling in HEK 293T cells stimulated with ligands such as $M$. avium extracts, $\mathrm{PAM}_{3} \mathrm{CSK}_{4}$ or FSL-1, which bind TLR2/1 or TLR2/6 heterodimers [Etokebe et al., 2010]. In addition, this variant has been shown to impair internalisation from the cell surface to early endosomal compartments [Etokebe et al., 2010]. For TLR6, five of the 17 non-synonymous variants segregating in the general population (p.Leu128Val, p.Leu194Pro, p.Ala474Val, p.Asn690Thr and p.Gln708His) displayed

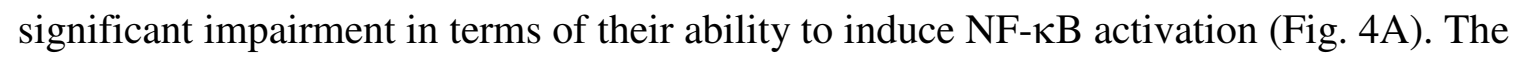
p.Leu128Val variant, which was the most strongly impaired, is located in the conserved "LxxLxLxxN" motif of LRR4 responsible for the characteristic horseshoe-like shape of the protein [Kang et al., 2009]. The other four variants - p.Leu194Pro and p.Ala474Val in the 
extracellular domain and p.Asn690Thr and p.Gln708His in the TIR domain — displayed more modest impairments of NF-אB activation (Fig. 4A). However, the actual decrease in $\mathrm{NF}-\kappa \mathrm{B}$ activation displayed by these five functionally relevant variants may be more pronounced than described here, due to the small amounts of endogenous TLR6 in recipient cells.

It is interesting to note that, despite similar protein length (Fig. 1), TLR2 displays a lower number of amino-acid changes (seven non-synonymous mutations) than TLR1 and TLR6 (17 non-synonymous mutations each), in accordance with a previous study [Georgel et al., 2009]. Three of the seven non-synonymous TLR2 variants strongly decreased NF- $\kappa$ B activation and were present at very low population frequencies. This suggests stronger selective constraints on amino-acid altering variation in TLR2 than in TLR1 and TLR6, a hypothesis formally supported by evolutionary genetics data across vertebrate species and in humans, showing that purifying selection has been stronger for TLR2 than for TLR1 and TLR6 [Barreiro et al., 2009; Roach et al., 2005]. In the context of the TLR1-2-6 family, evolutionary and functional data converge to suggest that TLR2 fulfils an essential function in early innate immune responses. TLR2, by heterodimerising with TLR1 or TLR6, presents a broader agonist repertoire, recognising a diverse set of pathogens [Takeuchi et al., 2001; Takeuchi et al., 2002]. In addition to its role as a prototypic cell surface-expressed TLR, TLR2 has also been shown to induce type-I IFN production when expressed in the endosomal compartment [Barbalat et al., 2009; Bauernfeind and Hornung, 2009]. Variants of the TLR2 gene may therefore affect not only proinflammatory responses, but also IFN production, further constraining the evolution of this gene.

Finally, the comparison between the PolyPhen predictive results and the actual, functional consequences of TLR variants showed that the vast majority of mutations predicted as "benign" do not substantially affect protein function ( $80 \%)$, at least in terms of NF-кB 
activation (Fig. 5A). Similarly, most mutations predicted as possibly or probably damaging considerably impact protein function $(\sim 73 \%)$. Although in most cases there is a good concordance between the PolyPhen predictions and the experimental observations, two exceptions are worth mentioning in this respect: TLR1 p.Pro315Leu and p.Ile602Ser. These two variants, which have been both convincingly shown to drastically reduce NF- $\kappa \mathrm{B}$ activation by us (Fig. 2A) and others [Barreiro et al., 2009; Hawn et al., 2007; Johnson et al., 2007; Omueti et al., 2007], are indeed predicted to be benign by PolyPhen (Table 1, Fig. 5A), highlighting some of the limitations of predictive methods. An interesting observation is that most of the variants either predicted to be damaging by PolyPhen or experimentally shown to impair NF- $\kappa \mathrm{B}$ activation are rare and population-specific, suggesting that considerable evolutionary pressure may maintain these variants at low population frequencies because of their probable deleterious effects. Most functionally relevant variants are singletons, but heterozygous individuals may have a higher risk of infection due to dominant negative or gene dosage effects, raising questions about the epidemiological relevance of rare TLR mutations. Indeed, associations between low-frequency TLR variants and infectious disease susceptibility have been reported for TLR4 and meningococcal susceptibility [Smirnova et al., 2003] and for TLR6-TLR1-TLR10 and tuberculosis [Ma et al., 2007].

In summary, rare, functionally impaired TLR variants may be major risk factors in the host affecting the outcome and/or severity of infection. They should therefore be considered in the context of large-scale association studies of genetic susceptibility to disease. Indeed, rare variants are not generally tested in traditional genome-wide association studies, which are principally based on the genotyping of common polymorphisms (i.e., frequencies $>5 \%$ ) present on commercial arrays. In this context, upcoming association studies based on wholeexome resequencing should compensate for this limitation and shed light on the ways in 
which rare, population-specific variants contribute to susceptibility to, or the pathogenesis of infectious diseases. 


\section{ACKNOWLEDGEMENTS}

We thank Josiane Ragimbeau and Sylvie Garcia for technical help, and Olivier Neyrolles and Dhafer Laouini for critical reading of the manuscript. This work was supported by the Institut Pasteur research program PTR202 “The Genetics of Host Predisposition to Infectious Diseases", the Centre National de la Recherche Scientifique (CNRS), Merck-Serono, and an Agence Nationale de la Recherche (ANR) research grant (ANR-05-JCJC-0124-01 to L.Q.M.). 


\section{References}

Adzhubei IA, Schmidt S, Peshkin L, Ramensky VE, Gerasimova A, Bork P, Kondrashov AS, Sunyaev SR. 2010. A method and server for predicting damaging missense mutations. Nat Methods 7(4):248-249.

Akira S, Takeda K. 2004. Toll-like receptor signalling. Nat Rev Immunol 4(7):499-511.

Akira S, Uematsu S, Takeuchi O. 2006. Pathogen recognition and innate immunity. Cell 124(4):783-801.

Barbalat R, Lau L, Locksley RM, Barton GM. 2009. Toll-like receptor 2 on inflammatory monocytes induces type I interferon in response to viral but not bacterial ligands. Nat Immunol 10(11):1200-1207.

Barreiro LB, Ben-Ali M, Quach H, Laval G, Patin E, Pickrell JK, Bouchier C, Tichit M, Neyrolles O, Gicquel B, Kidd JR, Kidd KK, Alcais A, Ragimbeau J, Pellegrini S, Abel L, Casanova JL, Quintana-Murci L. 2009. Evolutionary dynamics of human Toll-like receptors and their different contributions to host defense. PLoS Genet 5(7):e1000562.

Bauernfeind F, Hornung V. 2009. TLR2 joins the interferon gang. Nat Immunol 10(11):11391141.

Berdeli A, Celik HA, Ozyurek R, Dogrusoz B, Aydin HH. 2005. TLR-2 gene Arg753Gln polymorphism is strongly associated with acute rheumatic fever in children. J Mol Med 83(7):535-541.

Beutler B. 2004. Inferences, questions and possibilities in Toll-like receptor signalling. Nature 430(6996):257-263.

Beutler B, Hoebe K, Du X, Ulevitch RJ. 2003. How we detect microbes and respond to them: the Toll-like receptors and their transducers. J Leukoc Biol 74(4):479-485. 
Beutler B, Rehli M. 2002. Evolution of the TIR, tolls and TLRs: functional inferences from computational biology. Curr Top Microbiol Immunol 270:1-21.

Casanova JL, Abel L, Quintana-Murci L. 2011. Human TLRs and IL-1Rs in Host Defense: Natural Insights from Evolutionary, Epidemiological, and Clinical Genetics. Annu Rev Immunol.

Enard D, Depaulis F, Roest Crollius H. 2010. Human and non-human primate genomes share hotspots of positive selection. PLoS Genet 6(2):e1000840.

Etokebe GE, Skjeldal F, Nilsen N, Rodionov D, Knezevic J, Bulat-Kardum L, Espevik T, Bakke O, Dembic Z. 2010. Toll-like receptor 2 (P631H) mutant impairs membrane internalization and is a dominant negative allele. Scand J Immunol 71(5):369-381.

Garantziotis S, Hollingsworth JW, Zaas AK, Schwartz DA. 2008. The effect of toll-like receptors and toll-like receptor genetics in human disease. Annu Rev Med 59:343-359.

Georgel P, Macquin C, Bahram S. 2009. The heterogeneous allelic repertoire of human tolllike receptor (TLR) genes. PLoS One 4(11):e7803.

Gong J, Wei T, Zhang N, Jamitzky F, Heckl WM, Rossle SC, Stark RW. 2010. TollML: a database of toll-like receptor structural motifs. J Mol Model 16(7):1283-1289.

Hawn TR, Misch EA, Dunstan SJ, Thwaites GE, Lan NT, Quy HT, Chau TT, Rodrigues S, Nachman A, Janer M, Hien TT, Farrar JJ, Aderem A. 2007. A common human TLR1 polymorphism regulates the innate immune response to lipopeptides. Eur J Immunol 37(8):2280-2289.

Janeway CA, Jr., Medzhitov R. 2002. Innate immune recognition. Annu Rev Immunol 20:197-216.

Johnson CM, Lyle EA, Omueti KO, Stepensky VA, Yegin O, Alpsoy E, Hamann L, Schumann RR, Tapping RI. 2007. Cutting edge: A common polymorphism impairs 
cell surface trafficking and functional responses of TLR1 but protects against leprosy. J Immunol 178(12):7520-7524.

Kang JY, Nan X, Jin MS, Youn SJ, Ryu YH, Mah S, Han SH, Lee H, Paik SG, Lee JO. 2009. Recognition of lipopeptide patterns by Toll-like receptor 2-Toll-like receptor 6 heterodimer. Immunity 31(6):873-884.

Kawai T, Akira S. 2006. Innate immune recognition of viral infection. Nat Immunol 7(2):131137.

Kawai T, Akira S. 2007. TLR signaling. Semin Immunol 19(1):24-32.

Kijpittayarit S, Eid AJ, Brown RA, Paya CV, Razonable RR. 2007. Relationship between Toll-like receptor 2 polymorphism and cytomegalovirus disease after liver transplantation. Clin Infect Dis 44(10):1315-1320.

Kimbrell DA, Beutler B. 2001. The evolution and genetics of innate immunity. Nat Rev Genet 2(4):256-267.

Kormann MS, Ferstl R, Depner M, Klopp N, Spiller S, Illig T, Vogelberg C, von Mutius E, Kirschning CJ, Kabesch M. 2009. Rare TLR2 mutations reduce TLR2 receptor function and can increase atopy risk. Allergy 64(4):636-642.

Lemaitre B, Hoffmann J. 2007. The host defense of Drosophila melanogaster. Annu Rev Immunol 25:697-743.

Leulier F, Lemaitre B. 2008. Toll-like receptors--taking an evolutionary approach. Nat Rev Genet 9(3):165-178.

Ma X, Liu Y, Gowen BB, Graviss EA, Clark AG, Musser JM. 2007. Full-exon resequencing reveals toll-like receptor variants contribute to human susceptibility to tuberculosis disease. PLoS One 2(12):e1318.

Medzhitov R. 2001. Toll-like receptors and innate immunity. Nat Rev Immunol 1(2):135-145.

Medzhitov R, Janeway C, Jr. 2000. Innate immunity. N Engl J Med 343(5):338-344. 
Medzhitov R, Janeway CA, Jr. 1997. Innate immunity: the virtues of a nonclonal system of recognition. Cell 91(3):295-298.

Merx S, Neumaier M, Wagner H, Kirschning CJ, Ahmad-Nejad P. 2007. Characterization and investigation of single nucleotide polymorphisms and a novel TLR2 mutation in the human TLR2 gene. Hum Mol Genet 16(10):1225-1232.

Misch EA, Macdonald M, Ranjit C, Sapkota BR, Wells RD, Siddiqui MR, Kaplan G, Hawn TR. 2008. Human TLR1 Deficiency Is Associated with Impaired Mycobacterial Signaling and Protection from Leprosy Reversal Reaction. PLoS Negl Trop Dis 2(5):e231.

Ogus AC, Yoldas B, Ozdemir T, Uguz A, Olcen S, Keser I, Coskun M, Cilli A, Yegin O. 2004. The Arg753GLn polymorphism of the human toll-like receptor 2 gene in tuberculosis disease. Eur Respir J 23(2):219-223.

Okusawa T, Fujita M, Nakamura J, Into T, Yasuda M, Yoshimura A, Hara Y, Hasebe A, Golenbock DT, Morita M, Kuroki Y, Ogawa T, Shibata K. 2004. Relationship between structures and biological activities of mycoplasmal diacylated lipopeptides and their recognition by toll-like receptors 2 and 6. Infect Immun 72(3):1657-1665.

Omueti KO, Mazur DJ, Thompson KS, Lyle EA, Tapping RI. 2007. The polymorphism P315L of human toll-like receptor 1 impairs innate immune sensing of microbial cell wall components. J Immunol 178(10):6387-6394.

Ozinsky A, Underhill DM, Fontenot JD, Hajjar AM, Smith KD, Wilson CB, Schroeder L, Aderem A. 2000. The repertoire for pattern recognition of pathogens by the innate immune system is defined by cooperation between toll-like receptors. Proc Natl Acad Sci U S A 97(25):13766-13771. 
Pickrell JK, Coop G, Novembre J, Kudaravalli S, Li JZ, Absher D, Srinivasan BS, Barsh GS, Myers RM, Feldman MW, Pritchard JK. 2009. Signals of recent positive selection in a worldwide sample of human populations. Genome Res 19(5):826-837.

Roach JC, Glusman G, Rowen L, Kaur A, Purcell MK, Smith KD, Hood LE, Aderem A. 2005. The evolution of vertebrate Toll-like receptors. Proc Natl Acad Sci U S A 102(27):9577-9582.

Smirnova I, Mann N, Dols A, Derkx HH, Hibberd ML, Levin M, Beutler B. 2003. Assay of locus-specific genetic load implicates rare Toll-like receptor 4 mutations in meningococcal susceptibility. Proc Natl Acad Sci U S A 100(10):6075-6080.

Takahashi M, Matsuda F, Margetic N, Lathrop M. 2003. Automated identification of single nucleotide polymorphisms from sequencing data. J Bioinform Comput Biol 1(2):253265.

Takeuchi O, Kawai T, Muhlradt PF, Morr M, Radolf JD, Zychlinsky A, Takeda K, Akira S. 2001. Discrimination of bacterial lipoproteins by Toll-like receptor 6. Int Immunol 13(7):933-940.

Takeuchi O, Sato S, Horiuchi T, Hoshino K, Takeda K, Dong Z, Modlin RL, Akira S. 2002. Cutting edge: role of Toll-like receptor 1 in mediating immune response to microbial lipoproteins. J Immunol 169(1):10-14.

Tantisira K, Klimecki WT, Lazarus R, Palmer LJ, Raby BA, Kwiatkowski DJ, Silverman E, Vercelli D, Martinez FD, Weiss ST. 2004. Toll-like receptor 6 gene (TLR6): singlenucleotide polymorphism frequencies and preliminary association with the diagnosis of asthma. Genes Immun 5(5):343-346.

West AP, Koblansky AA, Ghosh S. 2006. Recognition and signaling by toll-like receptors. Annu Rev Cell Dev Biol 22:409-437. 
Wong SH, Gochhait S, Malhotra D, Pettersson FH, Teo YY, Khor CC, Rautanen A, Chapman SJ, Mills TC, Srivastava A, Rudko A, Freidin MB, Puzyrev VP, Ali S, Aggarwal S, Chopra R, Reddy BS, Garg VK, Roy S, Meisner S, Hazra SK, Saha B, Floyd S, Keating BJ, Kim C, Fairfax BP, Knight JC, Hill PC, Adegbola RA, Hakonarson H, Fine PE, Pitchappan RM, Bamezai RN, Hill AV, Vannberg FO. 2010. Leprosy and the adaptation of human toll-like receptor 1. PLoS Pathog 6:e1000979.

John Wiley \&-27- Sons, Inc. 


\section{Legends to Figures}

Figure 1: Naturally occurring non-synonymous variants in the TLR1-2-6 family. Nonsynonymous mutations in black, blue and orange correspond to those predicted to be benign, possibly damaging, and probably damaging, respectively. Mutations shown in red are nonsense mutations.

Figure 2. Functional analyses of TLR1 variants. (A) Dual luciferase assay of HEK293T cells transiently transfected with wild-type TLR1 and various naturally occurring variants. The amounts of plasmid DNA used for transfection were optimised so as to minimise baseline reporter activation with TLR2 alone (see Materials and Methods). The data shown are the means \pm standard deviations (SD) from three independent experiments each performed in triplicate, expressed as the percent relative firefly luciferase activity (RLU) (normalised with respect to Renilla luciferase activity). ${ }^{*} \mathrm{p}<0.05 * * \mathrm{p}<0.001$ (as determined by Student's $t$ test) for comparisons of the various TLR1 allelic variants with the wild type (ancestral form). (B) Cell-surface expression of TLR1 variants, assessed by flow cytometry analysis. Cells were transfected with $2 \mu \mathrm{g}$ of TLR1 expression vector (solid line) or the empty vector (dashed line), as indicated. After $48 \mathrm{~h}$, cells were detached and incubated with the anti-TLR1 mAbs GD2.F4. (C) Western-blot analyses of functionally relevant TLR1 variants. Cells were transfected with $100 \mathrm{ng}$ of HA-tagged ancestral TLR1, HA-tagged TLR1 variant or the empty vector.

Figure 3. Functional analyses of TLR2 variants. (A) Dual luciferase assay of HEK293T cells transiently transfected with wild-type TLR2 and various naturally occurring variants. The data shown are the means \pm SD for three independent experiments, each performed in triplicate, expressed as the RLU (normalised with respect to Renilla luciferase activity). * 
$\mathrm{p}<0.05 * * \mathrm{p}<0.001$ (as determined by Student's $t$ test) for comparisons of the various TLR2 allelic variants with the wild type (ancestral form). (B) Cell-surface expression of TLR1 variants, analysed by flow cytometry. Cells were transfected with $2 \mu \mathrm{g}$ of TLR2 vector (solid line), or the empty vector (dashed line) as indicated. After $48 \mathrm{~h}$, cells were detached and incubated with the anti-TLR2 mAbs T2.5. (C) Western-blot analyses of functionally relevant TLR2 variants. Cells were transfected with $100 \mathrm{ng}$ of HA-tagged ancestral TLR2, HA-tagged TLR2 variant or the empty vector.

Figure 4. Functional analyses of TLR6 variants. (A) Dual luciferase assay of HEK293T cells transiently transfected with wild-type TLR6 and various naturally occurring variants. The data shown are the means \pm SD for three independent experiments, each performed in triplicate, expressed as the RLU (normalised with respect to Renilla luciferase activity). * $p<0.05 * * p<0.001$ (as determined by Student's $t$ test) for comparison of the various TLR6 allelic variants with the wild type (ancestral form). For TLR2/6 P680H, "DN" indicates “dominant negative" mutation. (B) Western-blot analyses of functionally relevant TLR6 variants. Cells were transfected with $100 \mathrm{ng}$ of HA-tagged ancestral TLR6, HA-tagged TLR6 variant or the empty vector.

Figure 5. Functional impact of TLR1-2-6 variants. NF- $\kappa B$ activation impairment is presented with respect to (A) PolyPhen predictions and (B) protein domains. Variants predicted to be "damaging" have a more severe effect on NF- $\kappa \mathrm{B}$ activation than variants predicted to be "benign". Mutations affecting the TIR domain generally seem to have a greater impact on TLR signalling than those affecting the extracellular domain. 
Table 1. Description of the studied TLRI-2-6 gene family amino-acid altering variants

\begin{tabular}{|c|c|c|c|c|c|c|c|c|}
\hline \multirow[t]{2}{*}{ Gene } & \multirow[t]{2}{*}{ rs\# dbSNP } & \multirow[t]{2}{*}{$\operatorname{cSNP}^{\mathrm{a}}$} & \multicolumn{3}{|c|}{ Frequency $(\%)^{b}$} & \multirow[t]{2}{*}{ AA change ${ }^{c}$} & \multirow[t]{2}{*}{ PolyPhen-2 } & \multirow[t]{2}{*}{ NF-kB $(\%)^{d}$} \\
\hline & & & AFR & EUR & ASN & & & \\
\hline TLR1 & rs76600635 & c. $130 \mathrm{~T}>\mathrm{C}$ & 0 & 3.19 & 4.17 & p.Ser44Pro & benign & 102.54 \\
\hline TLR1 & New & c. $224 \mathrm{~T}>\mathrm{C}$ & 0.79 & 0 & 0 & p.Ile75Thr & possibly damaging & 125.38 \\
\hline TLR1 & rs5743611 & c. $239 \mathrm{G}>\mathrm{C}$ & 0 & 7.45 & 0 & p.Arg80Thr & benign & 94.62 \\
\hline TLR1 & rs5743612 & c. $352 \mathrm{C}>\mathrm{T}$ & 13.5 & 0 & 0 & p.His118Tyr & probably damaging & 101.50 \\
\hline TLR1 & rs4833095 & c. $743 \mathrm{G}>\mathrm{A}$ & 10.3 & 37.2 & 58.3 & p.Ser248Asn & benign & 91.12 \\
\hline TLR1 & rs3923647 & c. $914 \mathrm{~A}>\mathrm{T}$ & 3.97 & 2.13 & 0 & p.His305Leu & benign & 101.36 \\
\hline TLR1 & rs5743613 & c. $944 \mathrm{C}>\mathrm{T}$ & 0.79 & 0 & 0 & p.Pro315Leu & benign & 32.79 \\
\hline TLR1 & rs76796448 & c. $1054 \mathrm{C}>\mathrm{A}$ & 5.56 & 0 & 0 & p.His352Asn & benign & 103.24 \\
\hline TLR1 & New & c. $1378 \mathrm{~A}>\mathrm{G}$ & 0 & 0 & 1.04 & p.Ile460Val & benign & 112.79 \\
\hline TLR1 & New & c. $1625 \mathrm{~T}>\mathrm{C}$ & 0.79 & 0 & 0 & p.Val542Ala & benign & 96.25 \\
\hline TLR1 & New & c. $1661 \mathrm{~A}>\mathrm{G}$ & 0 & 0 & 1.04 & p.Tyr554Cys & probably damaging & 54.22 \\
\hline TLR1 & rs5743617 & c. $1760 \mathrm{~T}>\mathrm{G}$ & 5.56 & 0 & 0 & p.Val587Gly & benign & 101.67 \\
\hline TLR1 & rs5743618 & c. $1805 \mathrm{~T}>\mathrm{G}$ & 0 & 51.1 & 2.08 & p.Ile602Ser & benign & 45.08 \\
\hline TLR1 & New & c. $1952 \mathrm{~T}>\mathrm{C}$ & 1.59 & 0 & 0 & p.Val651Ala & probably damaging & 63.68 \\
\hline TLR1 & New & c. $2020 \mathrm{G}>\mathrm{A}$ & 0.79 & 0 & 0 & p.Val674Ala & benign & 111.19 \\
\hline TLR1 & rs113706342 & c. $2159 \mathrm{~A}>\mathrm{C}$ & 0 & 2.13 & 0 & p.His720Pro & probably damaging & 69.84 \\
\hline TLR1 & rs5743621 & c. $2198 \mathrm{C}>\mathrm{T}$ & 1.59 & 0 & 0 & p.Pro733Leu & probably damaging & 101.91 \\
\hline TLR2 & New & c. $265 \mathrm{~A}>\mathrm{G}$ & 0 & 1.06 & 0 & p.Asn89Asp & benign & 101.34 \\
\hline TLR2 & rs5743699 & c. $1232 \mathrm{C}>\mathrm{T}$ & 0 & 1.06 & 0 & p.Thr411Ile & possibly damaging & 44.46 \\
\hline TLR2 & rs62323857 & c. $1339 \mathrm{C}>\mathrm{T}$ & 0 & 1.06 & 0 & p.Arg447X & STOP & 9.10 \\
\hline TLR2 & rs61735277 & c. $1712 \mathrm{G}>\mathrm{A}$ & 0.79 & 0 & 0 & p.Arg571His & benign & 99.17 \\
\hline TLR2 & rs5743704 & c. $1892 \mathrm{C}>\mathrm{A}$ & 0 & 5.32 & 0 & p.Pro631His & probably damaging & 72.75 \\
\hline TLR2 & New & c. $1906 \mathrm{~A}>\mathrm{C}$ & 0.79 & 0 & 0 & p.Ser636Arg & benign & 110.84 \\
\hline TLR2 & rs5743708 & c. $2258 \mathrm{G}>\mathrm{A}$ & 0 & 2.13 & 0 & p.Arg753Gln & probably damaging & 26.00 \\
\hline TLR6 & rs5743808 & c. $359 \mathrm{~T}>\mathrm{C}$ & 10.3 & 0 & 1.04 & p.Ile120Thr & benign & 91.12 \\
\hline TLR6 & New & c. $382 \mathrm{C}>\mathrm{G}$ & 0 & 0 & 2.08 & p.Leu128Val & probably damaging & 58.49 \\
\hline TLR6 & rs5743809 & c. $581 \mathrm{~T}>\mathrm{C}$ & 7.14 & 0 & 0 & p.Leu194Pro & benign & 74.64 \\
\hline TLR6 & New & c. $628 \mathrm{G}>\mathrm{A}$ & 0 & 0 & 1.04 & p.Ala210Thr & benign & 87.64 \\
\hline TLR6 & New & c. $629 \mathrm{C}>\mathrm{G}$ & 0 & 0 & 1.04 & p.Ala210Gly & benign & 88.77 \\
\hline TLR6 & rs35220466 & c. $740 \mathrm{G}>\mathrm{A}$ & 3.97 & 0 & 0 & p.Arg247Lys & benign & 84.42 \\
\hline TLR6 & rs5743810 & c. $745 \mathrm{C}>\mathrm{T}$ & 2.38 & 28.7 & 0 & p.Pro249Ser & benign & 92.66 \\
\hline TLR6 & New & c. $847 \mathrm{~A}>\mathrm{G}$ & 0.79 & 0 & 0 & p.Ile283Val & benign & 95.27 \\
\hline TLR6 & rs 3796508 & c. $979 \mathrm{G}>\mathrm{A}$ & 1.59 & 0 & 1.04 & p.Val327Met & benign & 104.29 \\
\hline TLR6 & rs5743815 & c. $1280 \mathrm{~T}>\mathrm{C}$ & 0 & 1.06 & 0 & p.Val427Ala & benign & 91.00 \\
\hline TLR6 & New & c. $1325 \mathrm{~A}>\mathrm{C}$ & 0.79 & 0 & 0 & p.Asp442Ala & benign & 97.63 \\
\hline TLR6 & rs5743816 & c. $1393 \mathrm{G}>\mathrm{A}$ & 7.14 & 0 & 1.04 & p.Val465Ile & benign & 92.04 \\
\hline TLR6 & rs5743817 & c. $1420 \mathrm{G}>\mathrm{A}$ & 8.73 & 0 & 0 & p.Ala474Thr & benign & 88.58 \\
\hline TLR6 & rs35762983 & c. $1421 \mathrm{C}>\mathrm{T}$ & 0.79 & 0 & 0 & p.Ala474Val & benign & 77.30 \\
\hline TLR6 & rs5743617 & c. $1775 \mathrm{~T}>\mathrm{G}$ & 0 & 0 & 9.38 & p.Val592Gly & benign & 92.86 \\
\hline TLR6 & 1000 Genomes & c. $2069 A>C$ & 3.17 & 0 & 0 & p.Asn690Thr & benign & 72.28 \\
\hline TLR6 & New & c. $2124 \mathrm{G}>\mathrm{C}$ & 1.59 & 0 & 0 & p.Gln708His & probably damaging & 72.86 \\
\hline
\end{tabular}


${ }^{\text {a }}$ The first nucleotide corresponds to the ancestral state and the second to the derived allele, as defined on the basis of the chimpanzee sequence; ${ }^{b}$ Population allele frequency of the derived allele in sub-Saharan African (AFR), European (EUR) and Asian (ASN) populations; ${ }^{\mathrm{c}}$ The first amino-acid corresponds to the ancestral state; ${ }^{\mathrm{d}}$ Levels of NF- $\mathrm{KB}$ activation for each TLR variant with respect to the ancestral form (normalized at 100\%). In bold, functionally relevant variants (i.e. TLR variants with levels of NF- $\kappa B$ activation more than $20 \%$ lower than the ancestral form) 


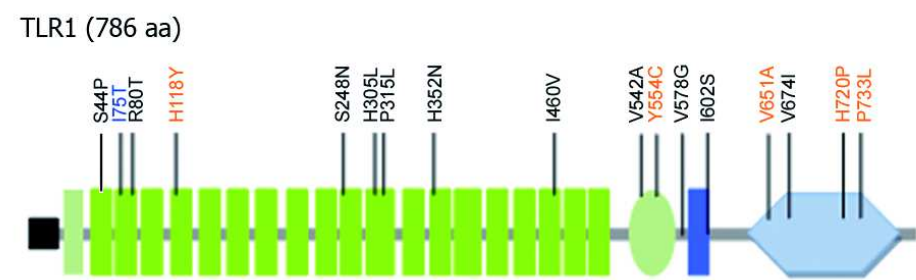

TLR2 (784 aa)

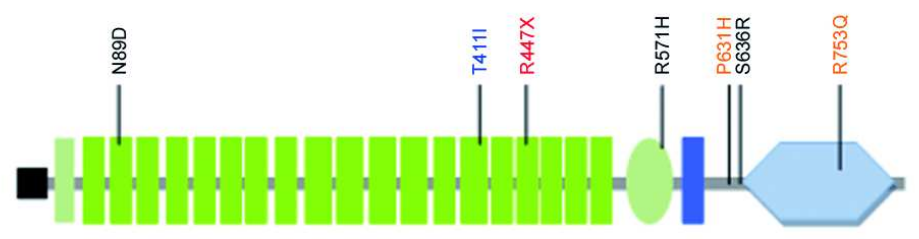

TLR6 (796 aa)
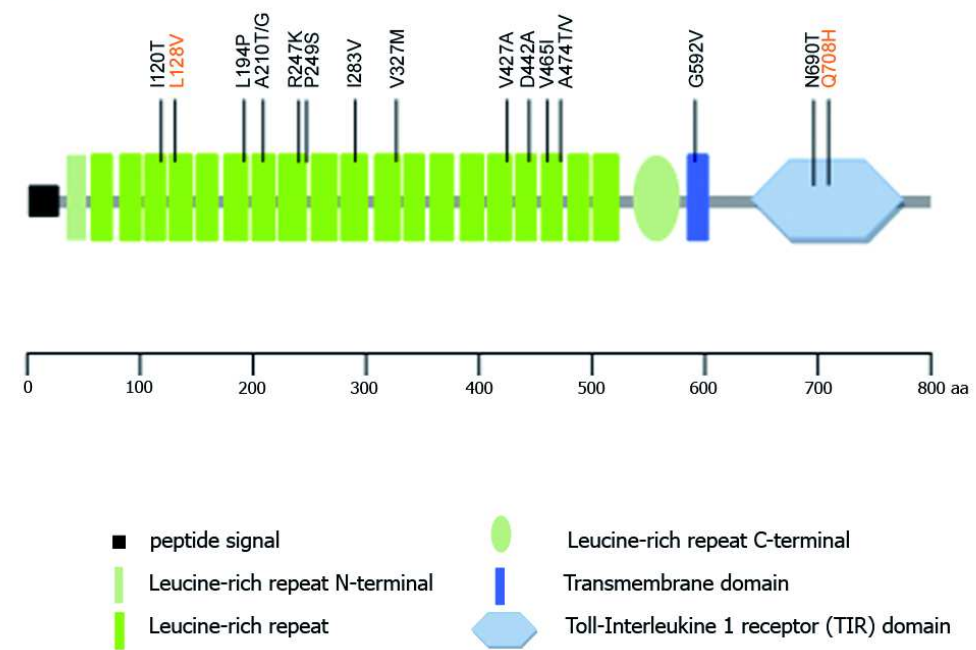

Leucine-rich repeat C-terminal

Transmembrane domain

Toll-Interleukine 1 receptor (TIR) domain

Naturally occurring non-synonymous variants in the TLR1-2-6 family $86 \times 129 \mathrm{~mm}(300 \times 300$ DPI)

John Wiley \& Sons, Inc. 
TLR1 (786 aa)

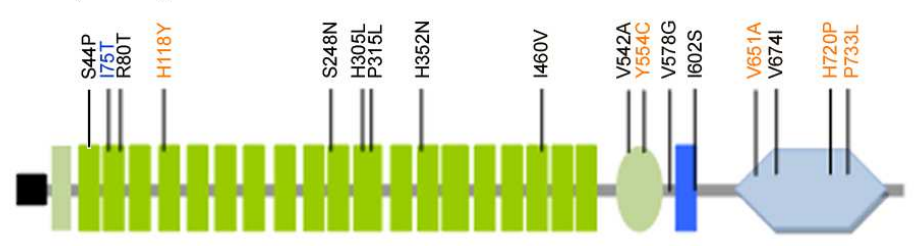

TLR2 (784 aa)

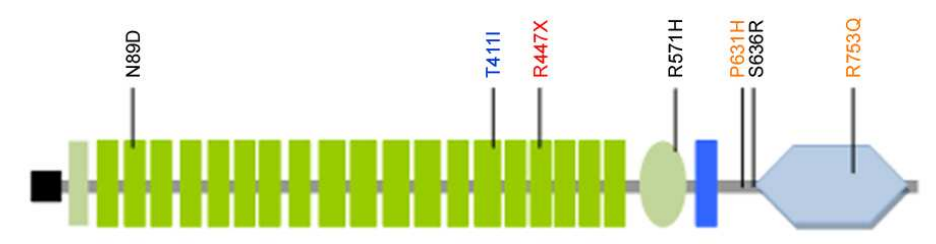

TLR6 (796 aa)
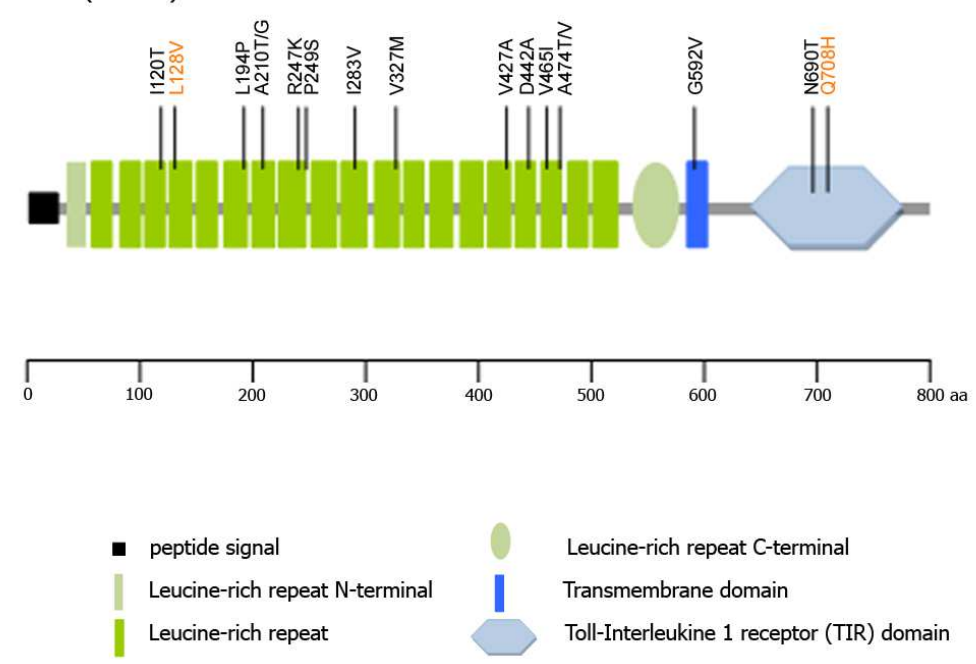

Naturally occurring non-synonymous variants in the TLR1-2-6 family $86 \times 129 \mathrm{~mm}(300 \times 300$ DPI)

John Wiley \& Sons, Inc. 
A

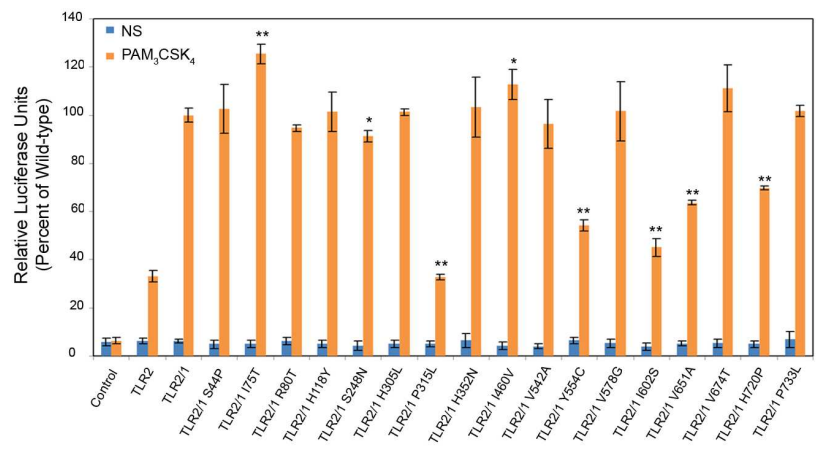

C

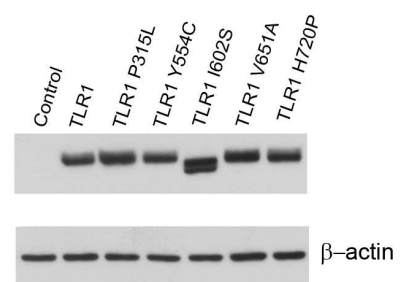

B

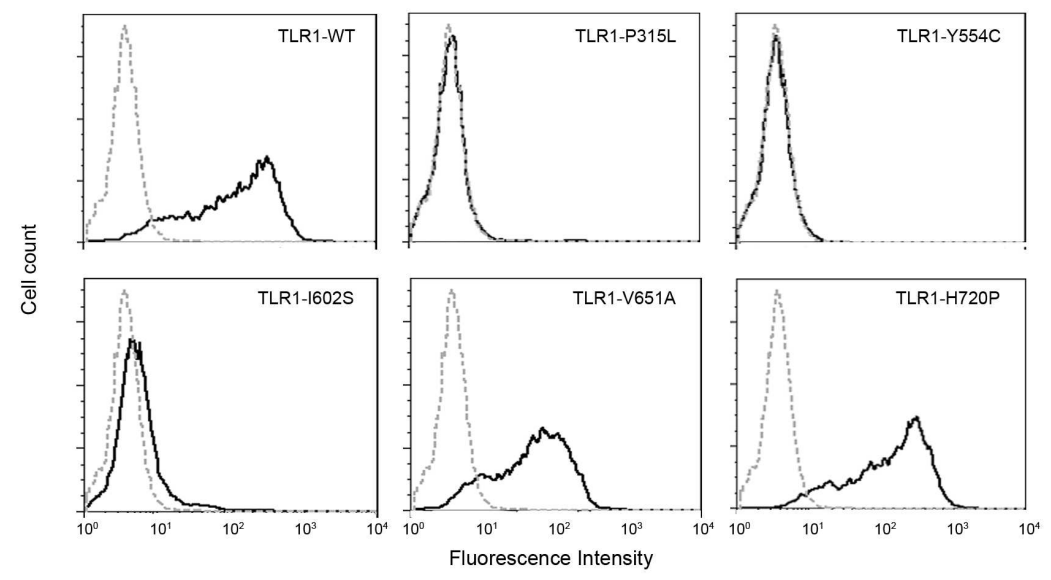

Functional analyses of TLR1 variants $180 \times 160 \mathrm{~mm}$ ( $300 \times 300$ DPI)

John Wiley \& Sons, Inc. 
A

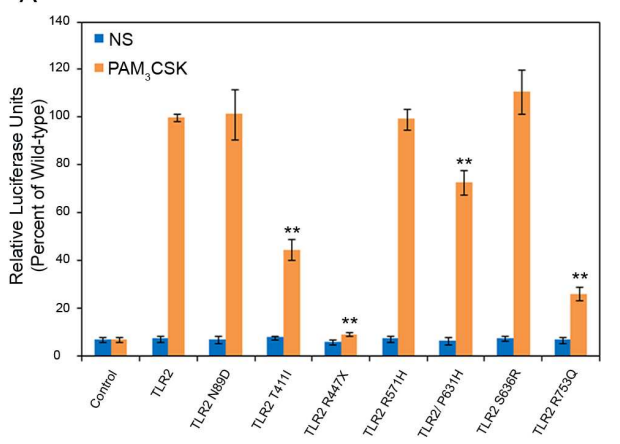

B

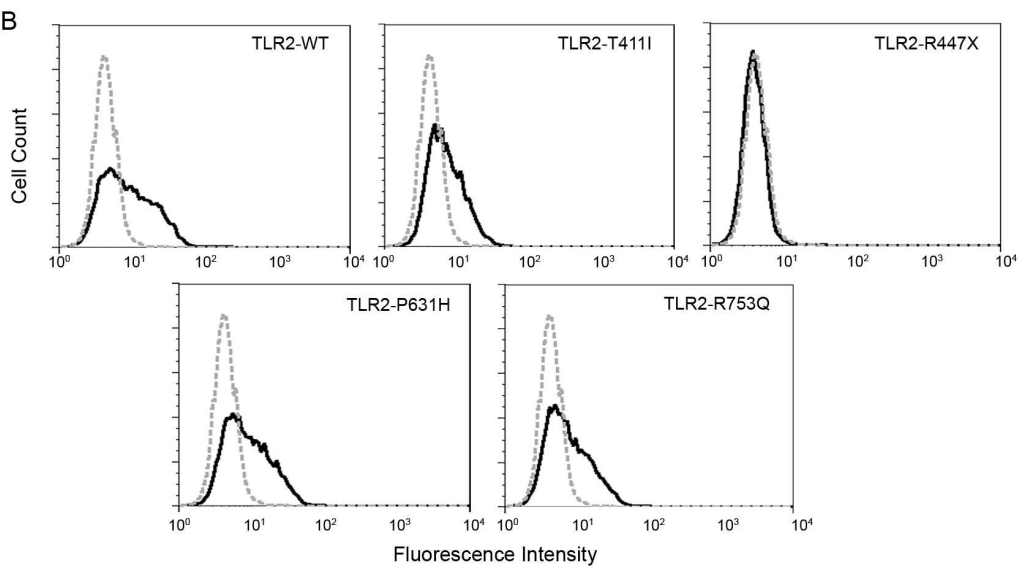

B

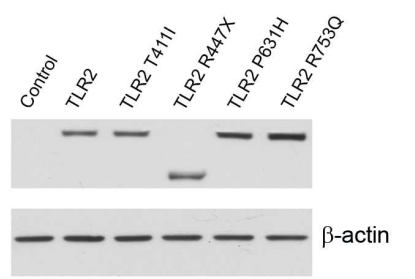

Functional analyses of TLR2 variants $180 \times 160 \mathrm{~mm}(300 \times 300 \mathrm{DPI})$

John Wiley \& Sons, Inc. 


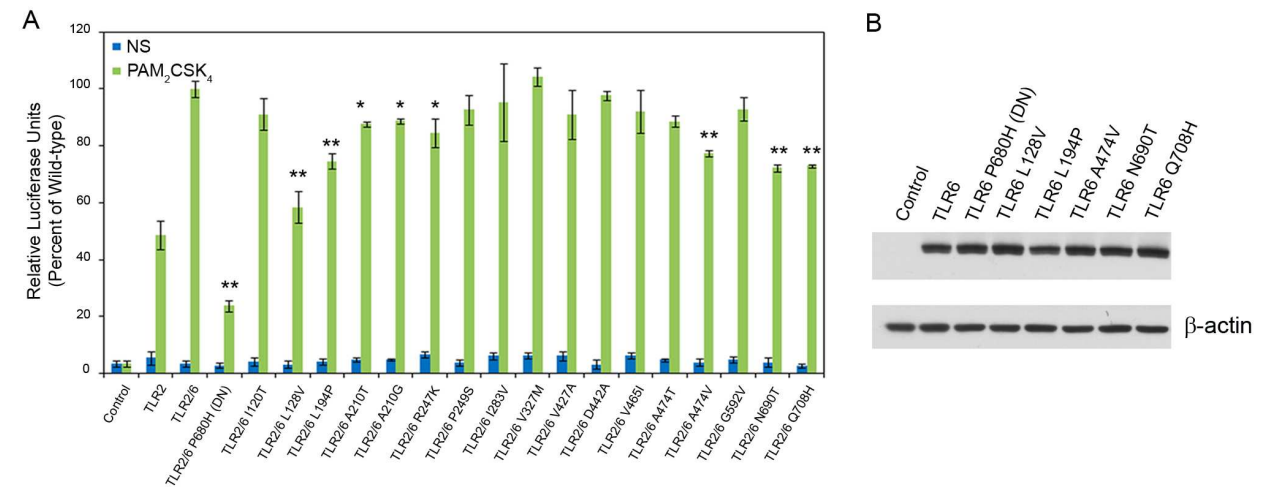

Functional analyses of TLR6 variants $180 \times 83 \mathrm{~mm}(300 \times 300$ DPI $)$

John Wiley \& Sons, Inc. 


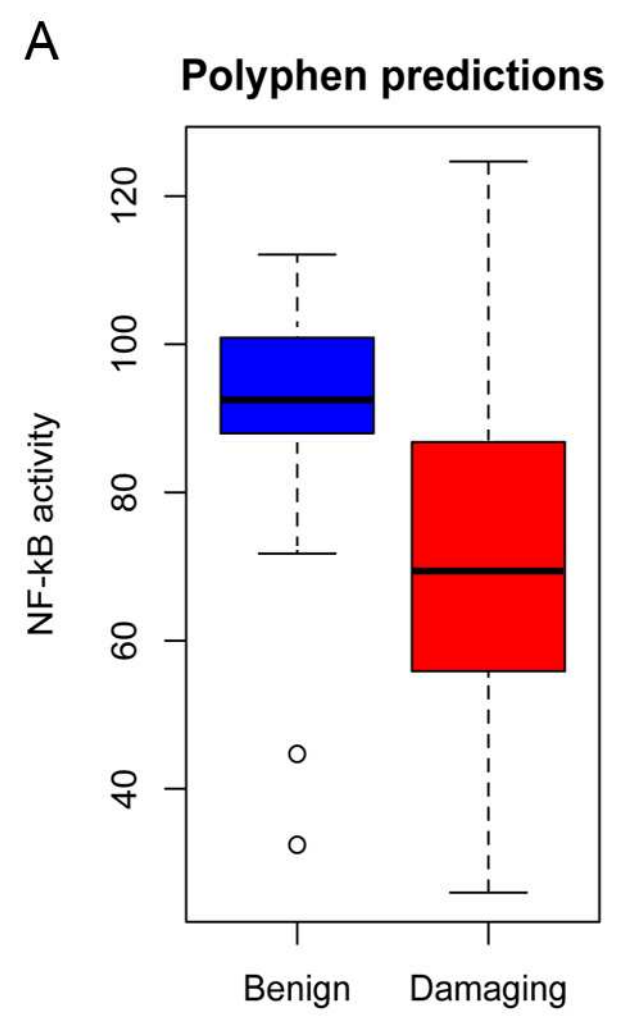

B Protein domains

Functional impact of TLR1-2-6 variants $86 \times 67 \mathrm{~mm}(300 \times 300$ DPI $)$ 


\section{Supporting Information}

"Functional characterisation of naturally occurring genetic variants in the human TLR1-2-6 gene family"

Supp. Table S1. Genotype information per individual of the TLR1 non-synonymous variants

\begin{tabular}{|c|c|c|c|c|c|c|c|c|c|c|c|c|c|c|c|c|c|c|c|}
\hline & & & SNP & SNP & SNP & SNP & SNP & SNP & SNP & SNP & SNP & SNP & SNP & SNP & SNP & SNP & SNP & SNP & SNP \\
\hline $\begin{array}{c}\begin{array}{c}\text { Contin } \\
\text { ent }\end{array} \\
\end{array}$ & $\begin{array}{l}\text { Populat } \\
\text { ion }\end{array}$ & ID & $\begin{array}{c}\text { c.130T } \\
>C\end{array}$ & $\begin{array}{c}\text { c.224T } \\
>C\end{array}$ & $\begin{array}{c}\text { c.239G } \\
>C\end{array}$ & $\begin{array}{c}c .352 \\
C>T\end{array}$ & $\begin{array}{c}\text { c.743G } \\
>A\end{array}$ & $\begin{array}{l}c .914 \\
A>T\end{array}$ & $\begin{array}{l}\text { c.944 } \\
\text { C>T }\end{array}$ & $\begin{array}{c}c .1054 \\
C>A\end{array}$ & $\begin{array}{c}c .1378 \\
A>G\end{array}$ & $\begin{array}{c}\text { C.1625 } \\
\text { T>C }\end{array}$ & $\begin{array}{c}c .1661 \\
A>G\end{array}$ & $\begin{array}{c}c .1760 \\
T>G\end{array}$ & $\begin{array}{c}\mathrm{c} .1805 \\
\mathrm{~T}>\mathrm{G}\end{array}$ & $\begin{array}{c}\mathrm{C} .1952 \\
\mathrm{~T}>\mathrm{C}\end{array}$ & $\begin{array}{c}c .2020 \\
G>A\end{array}$ & $\begin{array}{c}c .2159 \\
A>C\end{array}$ & $\begin{array}{c}c .2198 \\
C>T\end{array}$ \\
\hline $\begin{array}{c}\text { AFRIC } \\
\text { A }\end{array}$ & $\begin{array}{c}\text { Chagg } \\
a\end{array}$ & $\begin{array}{c}\text { CHA_JK } \\
4125\end{array}$ & $T$ & $\mathrm{~T}$ & $\mathrm{G}$ & $\mathrm{C}$ & $\mathrm{G}$ & $A$ & $\mathrm{C}$ & $A$ & $A$ & $T$ & $A$ & $T$ & $\mathrm{~T}$ & $\mathrm{~T}$ & $\mathrm{G}$ & $A$ & C \\
\hline $\begin{array}{c}\text { AFRIC } \\
\text { A }\end{array}$ & $\begin{array}{c}\text { Chagg } \\
a\end{array}$ & $\begin{array}{c}\text { CHA_JK } \\
4125 \\
\end{array}$ & $\bar{T}$ & $\bar{T}$ & $\bar{G}$ & $T$ & $\bar{G}$ & $\bar{A}$ & C & $C$ & $\bar{A}$ & $\bar{T}$ & $\bar{A}$ & $\bar{T}$ & $\bar{T}$ & $T$ & $\mathrm{G}$ & $A$ & C \\
\hline $\begin{array}{c}\text { AFRIC } \\
\text { A }\end{array}$ & $\begin{array}{c}\text { Chagg } \\
\text { a }\end{array}$ & $\begin{array}{c}\text { CHA_JK } \\
4126\end{array}$ & $\mathrm{~T}$ & $T$ & $\mathrm{G}$ & $C$ & $\mathrm{G}$ & $\bar{A}$ & $C$ & C & A & $T$ & $\bar{A}$ & $T$ & $T$ & $T$ & $\mathrm{G}$ & A & C \\
\hline $\begin{array}{c}\text { AFRIC } \\
\text { A }\end{array}$ & $\begin{array}{c}\text { Chagg } \\
\text { a }\end{array}$ & $\begin{array}{c}\text { CHA_JK } \\
4126\end{array}$ & $\mathrm{~T}$ & $\mathrm{~T}$ & $\mathrm{G}$ & $C$ & $\mathrm{G}$ & $\mathrm{A}$ & $C$ & $C$ & $\mathrm{~A}$ & $\mathrm{~T}$ & $\mathrm{~A}$ & $\mathrm{~T}$ & $\mathrm{~T}$ & $\mathrm{~T}$ & $\bar{G}$ & $\mathrm{~A}$ & $\mathrm{C}$ \\
\hline $\begin{array}{c}\text { AFRIC } \\
\text { A }\end{array}$ & $\begin{array}{c}\text { Chagg } \\
a\end{array}$ & $\begin{array}{c}\text { CHA_JK } \\
4127 \\
\end{array}$ & $T$ & $T$ & $\bar{G}$ & $C$ & $\bar{G}$ & A & $C$ & $C$ & A & $\mathrm{T}$ & A & $T$ & $\mathrm{~T}$ & $\mathrm{~T}$ & $\mathrm{G}$ & $A$ & $\mathrm{~T}$ \\
\hline $\begin{array}{c}\text { AFRIC } \\
\text { A }\end{array}$ & $\begin{array}{c}\text { Chagg } \\
\text { a }\end{array}$ & $\begin{array}{c}\text { CHA_JK } \\
4127\end{array}$ & $\mathrm{~T}$ & $T$ & $\bar{G}$ & C & $\bar{G}$ & $\bar{A}$ & C & C & $\bar{A}$ & $\mathrm{~T}$ & $\bar{A}$ & $\mathrm{~T}$ & $\mathrm{~T}$ & $\mathrm{~T}$ & $G$ & $\bar{A}$ & C \\
\hline $\begin{array}{c}\text { AFRIC } \\
\text { A }\end{array}$ & $\begin{array}{c}\text { Chagg } \\
\text { a }\end{array}$ & $\begin{array}{c}\text { CHA_JK } \\
4128 \\
\end{array}$ & $T$ & $T$ & $\mathrm{G}$ & C & $A$ & $\mathrm{~A}$ & $C$ & C & $\bar{A}$ & $T$ & $A$ & $G$ & $\mathrm{~T}$ & $\mathrm{~T}$ & $G$ & A & C \\
\hline $\begin{array}{c}\text { AFRIC } \\
\text { A }\end{array}$ & $\begin{array}{c}\text { Chagg } \\
a\end{array}$ & $\begin{array}{c}\text { CHA_JK } \\
4128\end{array}$ & $\mathrm{~T}$ & $T$ & $\bar{G}$ & C & $\bar{G}$ & $\bar{A}$ & C & C & $\bar{A}$ & $\bar{T}$ & $\bar{A}$ & $T$ & $T$ & $\bar{T}$ & $\bar{G}$ & $\bar{A}$ & $C$ \\
\hline $\begin{array}{c}\text { AFRIC } \\
\text { A } \\
\end{array}$ & $\begin{array}{c}\text { Chagg } \\
a\end{array}$ & $\begin{array}{c}\text { CHA_JK } \\
4129\end{array}$ & $\mathrm{~T}$ & $T$ & $G$ & $C$ & $G$ & A & $C$ & A & A & $T$ & $A$ & $T$ & $\mathrm{~T}$ & $\mathrm{~T}$ & $\mathrm{G}$ & A & $C$ \\
\hline $\begin{array}{c}\text { AFRIC } \\
\text { A }\end{array}$ & $\begin{array}{c}\text { Chagg } \\
\text { a }\end{array}$ & $\begin{array}{c}\text { CHA_JK } \\
4129\end{array}$ & $\mathrm{~T}$ & $T$ & $\bar{G}$ & $C$ & $\bar{G}$ & $\bar{A}$ & C & $C$ & $\bar{A}$ & $T$ & $\bar{A}$ & $T$ & $\mathrm{~T}$ & $\mathrm{~T}$ & $\mathrm{G}$ & $\bar{A}$ & C \\
\hline $\begin{array}{c}\text { AFRIC } \\
\text { A }\end{array}$ & $\begin{array}{c}\text { Chagg } \\
\text { a }\end{array}$ & $\begin{array}{c}\text { CHA_JK } \\
4130\end{array}$ & $\mathrm{~T}$ & $\mathrm{~T}$ & $\mathrm{G}$ & $C$ & $\mathrm{G}$ & $\mathrm{A}$ & $C$ & $C$ & $\mathrm{~A}$ & $\mathrm{~T}$ & $\mathrm{~A}$ & $\mathrm{~T}$ & $\mathrm{~T}$ & $\mathrm{~T}$ & $G$ & $\mathrm{~A}$ & $C$ \\
\hline $\begin{array}{c}\text { AFRIC } \\
\text { A }\end{array}$ & $\begin{array}{c}\text { Chagg } \\
a\end{array}$ & $\begin{array}{c}\text { CHA_JK } \\
4130\end{array}$ & $\mathrm{~T}$ & $T$ & $\bar{G}$ & C & $\bar{G}$ & $\bar{A}$ & $\bar{C}$ & C & $\bar{A}$ & $T$ & $\bar{A}$ & $T$ & $\mathrm{~T}$ & $\mathrm{~T}$ & $\bar{G}$ & $\bar{A}$ & C \\
\hline $\begin{array}{c}\text { AFRIC } \\
\text { A }\end{array}$ & $\begin{array}{c}\text { Chagg } \\
\text { a }\end{array}$ & $\begin{array}{c}\text { CHA_JK } \\
4131\end{array}$ & $\mathrm{~T}$ & $T$ & $G$ & C & $G$ & $A$ & C & $C$ & $\bar{A}$ & $T$ & A & $T$ & $T$ & $\mathrm{~T}$ & $\mathrm{G}$ & A & $C$ \\
\hline $\begin{array}{c}\text { AFRIC } \\
\text { A }\end{array}$ & $\begin{array}{c}\text { Chagg } \\
\text { a }\end{array}$ & $\begin{array}{c}\text { CHA_JK } \\
4131\end{array}$ & $\mathrm{~T}$ & $\mathrm{~T}$ & $\mathrm{G}$ & $C$ & $\mathrm{G}$ & $\mathrm{T}$ & $C$ & $C$ & $\mathrm{~A}$ & $\mathrm{~T}$ & $\mathrm{~A}$ & $\mathrm{~T}$ & $\mathrm{~T}$ & $\mathrm{~T}$ & $\mathrm{G}$ & $\mathrm{A}$ & $C$ \\
\hline $\begin{array}{c}\text { AFRIC } \\
\text { A } \\
\end{array}$ & $\begin{array}{c}\text { Chagg } \\
a\end{array}$ & $\begin{array}{c}\text { CHA_JK } \\
4132 \\
\end{array}$ & $T$ & $\mathrm{~T}$ & $\mathrm{G}$ & $C$ & $A$ & $A$ & $C$ & $C$ & $A$ & $T$ & $\mathrm{~A}$ & $\mathrm{~T}$ & $\mathrm{~T}$ & $\mathrm{~T}$ & $\mathrm{G}$ & $A$ & $C$ \\
\hline $\begin{array}{c}\text { AFRIC } \\
\text { A }\end{array}$ & $\begin{array}{c}\text { Chagg } \\
\text { a }\end{array}$ & $\begin{array}{c}\text { CHA_JK } \\
4132\end{array}$ & $\mathrm{~T}$ & $\mathrm{~T}$ & $\bar{G}$ & C & $\bar{G}$ & $\bar{A}$ & $C$ & C & $\bar{A}$ & $T$ & $\bar{A}$ & $\mathrm{~T}$ & $T$ & $\mathrm{~T}$ & $\bar{G}$ & $\bar{A}$ & $C$ \\
\hline $\begin{array}{c}\text { AFRIC } \\
\text { A } \\
\end{array}$ & $\begin{array}{c}\text { Chagg } \\
\text { a }\end{array}$ & $\begin{array}{c}\text { CHA_JK } \\
4133\end{array}$ & $T$ & $T$ & $\mathrm{G}$ & $C$ & $\mathrm{G}$ & $A$ & $C$ & $C$ & A & $\mathrm{T}$ & A & $T$ & $T$ & $T$ & $\mathrm{G}$ & A & $C$ \\
\hline AFRIC & Chagg & CHA_JK & $\mathrm{T}$ & $\bar{T}$ & $\bar{G}$ & $T$ & $\bar{G}$ & $\bar{A}$ & $\mathrm{C}$ & $\mathrm{C}$ & A & $\mathrm{T}$ & $\bar{A}$ & $T$ & $\mathrm{~T}$ & $\mathrm{~T}$ & $\bar{G}$ & $\bar{A}$ & 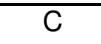 \\
\hline
\end{tabular}

John Wiley \& Sons, Inc. 


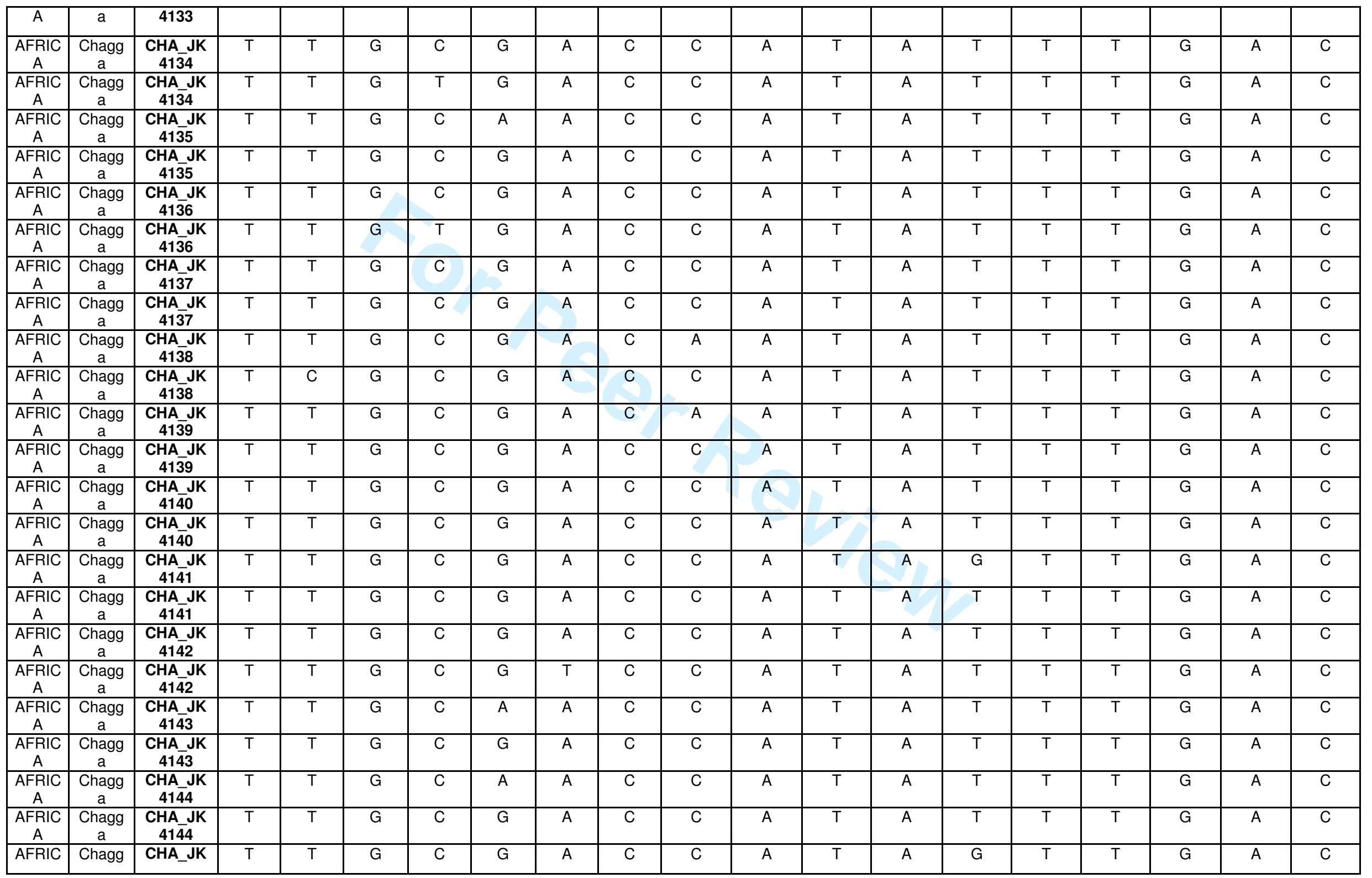

John Wiley ${ }^{2} \&$ Sons, Inc. 


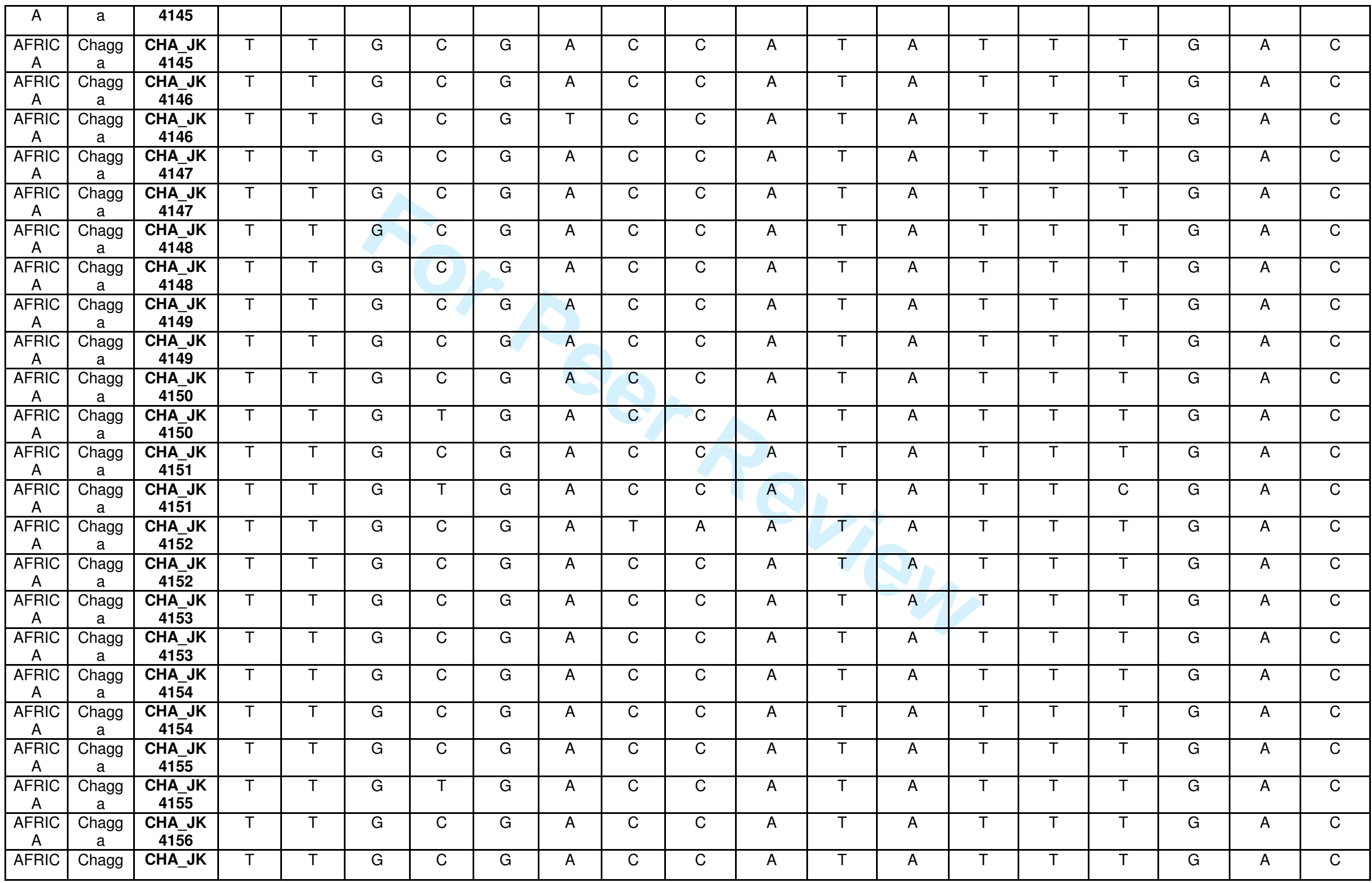

John Wiley ${ }^{3}$ \& Sons, Inc. 


\begin{tabular}{|c|c|c|c|c|c|c|c|c|c|c|c|c|c|c|c|c|c|c|c|}
\hline $\bar{A}$ & $\bar{a}$ & 4156 & & & & & & & & & & & & & & & & & \\
\hline $\begin{array}{c}\text { AFRIC } \\
\text { A }\end{array}$ & Yoruba & $\begin{array}{c}\text { YRB_JK3 } \\
878 \\
\end{array}$ & $\mathrm{~T}$ & $\bar{T}$ & $G$ & C & $\mathrm{G}$ & $A$ & $C$ & $A$ & $A$ & $\mathrm{~T}$ & A & $\mathrm{T}$ & $\mathrm{T}$ & $\mathrm{T}$ & $\mathrm{G}$ & A & $C$ \\
\hline $\begin{array}{c}\text { AFRIC } \\
\text { A }\end{array}$ & Yoruba & $\begin{array}{c}\text { YRB_JK3 } \\
878\end{array}$ & $\bar{T}$ & $\bar{T}$ & $G$ & $\mathrm{~T}$ & $\mathrm{G}$ & $A$ & $\mathrm{C}$ & $C$ & $A$ & $\mathrm{~T}$ & $\bar{A}$ & $\bar{T}$ & $\mathrm{~T}$ & $\bar{T}$ & $\mathrm{G}$ & $A$ & $\mathrm{C}$ \\
\hline $\begin{array}{c}\text { AFRIC } \\
\text { A }\end{array}$ & Yoruba & $\begin{array}{c}\text { YRB_JK3 } \\
879\end{array}$ & $\mathrm{~T}$ & $\mathrm{~T}$ & $\mathrm{G}$ & $C$ & $\mathrm{G}$ & A & C & $C$ & A & $\mathrm{T}$ & $A$ & $\mathrm{~T}$ & $\mathrm{~T}$ & $\mathrm{~T}$ & $G$ & A & C \\
\hline $\begin{array}{c}\text { AFRIC } \\
\text { A }\end{array}$ & Yoruba & $\begin{array}{c}\text { YRB_JK3 } \\
879\end{array}$ & $\mathrm{~T}$ & $\mathrm{~T}$ & $G$ & C & $\mathrm{G}$ & $A$ & $C$ & $C$ & $A$ & $\mathrm{~T}$ & $A$ & $\mathrm{~T}$ & $\mathrm{~T}$ & $\mathrm{~T}$ & $\mathrm{G}$ & $A$ & C \\
\hline $\begin{array}{c}\text { AFRIC } \\
\text { A }\end{array}$ & Yoruba & $\begin{array}{c}\text { YRB_JK3 } \\
880\end{array}$ & $\bar{T}$ & $\bar{T}$ & $G$ & C & $\mathrm{G}$ & $A$ & $\mathrm{C}$ & $C$ & $A$ & $\mathrm{~T}$ & $A$ & $\mathrm{G}$ & $\mathrm{T}$ & $\mathrm{T}$ & $\mathrm{G}$ & $A$ & $\mathrm{C}$ \\
\hline $\begin{array}{c}\text { AFRIC } \\
\text { A }\end{array}$ & Yoruba & $\begin{array}{c}\text { YRB_JK3 } \\
880\end{array}$ & $\mathrm{~T}$ & $\mathrm{~T}$ & $G$ & $C$ & $\mathrm{G}$ & A & C & $C$ & $A$ & $T$ & A & $\mathrm{T}$ & $T$ & $T$ & $G$ & A & $C$ \\
\hline $\begin{array}{c}\text { AFRIC } \\
\text { A }\end{array}$ & Yoruba & $\begin{array}{c}\text { YRB_JK3 } \\
881\end{array}$ & $\bar{T}$ & $\mathrm{~T}$ & $G$ & C & $\mathrm{G}$ & $A$ & $\mathrm{C}$ & $C$ & $A$ & $\mathrm{~T}$ & $A$ & $\mathrm{~T}$ & $\mathrm{~T}$ & $T$ & $\mathrm{G}$ & $A$ & C \\
\hline $\begin{array}{c}\text { AFRIC } \\
\text { A }\end{array}$ & Yoruba & $\begin{array}{c}\text { YRB_JK3 } \\
881\end{array}$ & $T$ & $T$ & $G$ & $\mathrm{C}$ & $\mathrm{G}$ & $A$ & C & $C$ & $A$ & $\mathrm{C}$ & $A$ & $\mathrm{~T}$ & $\mathrm{~T}$ & $\mathrm{~T}$ & $\mathrm{G}$ & $A$ & $C$ \\
\hline $\begin{array}{c}\text { AFRIC } \\
\text { A }\end{array}$ & Yoruba & $\begin{array}{c}\text { YRB_JK3 } \\
882\end{array}$ & $T$ & $T$ & $\bar{G}$ & $\mathrm{C}$ & $\mathrm{G}$ & $A$ & C & $C$ & $A$ & $\mathrm{~T}$ & $\bar{A}$ & $T$ & $\mathrm{~T}$ & $T$ & $\mathrm{G}$ & $A$ & C \\
\hline $\begin{array}{c}\text { AFRIC } \\
\text { A }\end{array}$ & Yoruba & $\begin{array}{c}\text { YRB_JK3 } \\
882\end{array}$ & $\mathrm{~T}$ & $\mathrm{~T}$ & $G$ & $\mathrm{~T}$ & $\mathrm{G}$ & A & C & $C$ & A & $\mathrm{T}$ & A & $\mathrm{T}$ & $\mathrm{T}$ & $T$ & $\mathrm{G}$ & A & C \\
\hline $\begin{array}{c}\text { AFRIC } \\
\text { A }\end{array}$ & Yoruba & $\begin{array}{c}\text { YRB_JK3 } \\
883\end{array}$ & $\mathrm{~T}$ & $T$ & $\mathrm{G}$ & C & $\mathrm{G}$ & $\bar{A}$ & $\mathrm{C}$ & $\mathrm{C}$ & $\bar{A}$ & $\bar{T}$ & $\bar{A}$ & $\mathrm{~T}$ & $\bar{T}$ & $\mathrm{~T}$ & $\mathrm{G}$ & $\mathrm{A}$ & $C$ \\
\hline $\begin{array}{c}\text { AFRIC } \\
\text { A }\end{array}$ & Yoruba & $\begin{array}{c}\text { YRB_JK3 } \\
883\end{array}$ & $\bar{T}$ & $\mathrm{~T}$ & $\bar{G}$ & $\mathrm{C}$ & $\mathrm{G}$ & $\bar{A}$ & $\mathrm{C}$ & $C$ & $\bar{A}$ & $\mathrm{~T}$ & $A$ & $\mathrm{~T}$ & $\mathrm{~T}$ & $\mathrm{~T}$ & $\mathrm{G}$ & $A$ & $C$ \\
\hline $\begin{array}{c}\text { AFRIC } \\
\text { A }\end{array}$ & Yoruba & $\begin{array}{c}\text { YRB_JK3 } \\
884\end{array}$ & $\mathrm{~T}$ & $\mathrm{~T}$ & $G$ & C & $\mathrm{G}$ & A & C & $C$ & A & $\mathrm{T}$ & A & $\mathrm{G}$ & $\bar{T}$ & $\bar{T}$ & $G$ & $A$ & C \\
\hline $\begin{array}{c}\text { AFRIC } \\
\text { A }\end{array}$ & Yoruba & $\begin{array}{c}\text { YRB_JK3 } \\
884\end{array}$ & $\mathrm{~T}$ & $T$ & $\mathrm{G}$ & $\bar{T}$ & $\mathrm{G}$ & $\bar{A}$ & $\bar{C}$ & C & $A$ & $T$ & $\bar{A}$ & $\mathrm{~T}$ & $\bar{T}$ & $T$ & $\mathrm{G}$ & $\bar{A}$ & C \\
\hline $\begin{array}{c}\text { AFRIC } \\
\text { A }\end{array}$ & Yoruba & $\begin{array}{c}\text { YRB_JK3 } \\
885\end{array}$ & $T$ & $\mathrm{~T}$ & $\bar{G}$ & $\mathrm{C}$ & $A$ & $A$ & $\mathrm{C}$ & $C$ & $A$ & $T$ & $\bar{A}$ & $\mathrm{~T}$ & $\bar{T}$ & $\bar{T}$ & $\mathrm{G}$ & $\bar{A}$ & $C$ \\
\hline $\begin{array}{c}\text { AFRIC } \\
\text { A }\end{array}$ & Yoruba & $\begin{array}{c}\text { YRB_JK3 } \\
885\end{array}$ & $\mathrm{~T}$ & $\mathrm{~T}$ & $G$ & C & $\mathrm{G}$ & A & $C$ & $C$ & A & $\mathrm{T}$ & A & $\mathrm{T}$ & $\bar{T}$ & $\bar{T}$ & $G$ & $A$ & C \\
\hline $\begin{array}{c}\text { AFRIC } \\
\text { A }\end{array}$ & Yoruba & $\begin{array}{c}\text { YRB_JK3 } \\
886\end{array}$ & $T$ & $T$ & $\mathrm{G}$ & C & $\mathrm{G}$ & $\bar{A}$ & C & $C$ & $A$ & $T$ & $A$ & $\mathrm{G}$ & $T$ & $\bar{T}$ & $\mathrm{G}$ & A & $C$ \\
\hline $\begin{array}{c}\text { AFRIC } \\
\text { A }\end{array}$ & Yoruba & $\begin{array}{c}\text { YRB_JK3 } \\
886\end{array}$ & $T$ & $T$ & $\mathrm{G}$ & C & $G$ & $\bar{A}$ & C & $C$ & $A$ & $T$ & $\bar{A}$ & $\mathrm{~T}$ & $T$ & $T$ & $\mathrm{G}$ & A & $C$ \\
\hline $\begin{array}{c}\text { AFRIC } \\
\text { A }\end{array}$ & Yoruba & $\begin{array}{c}\text { YRB_JK3 } \\
887\end{array}$ & $\mathrm{~T}$ & $\mathrm{~T}$ & $\mathrm{G}$ & C & A & A & $C$ & $C$ & $A$ & $T$ & A & $\mathrm{T}$ & $\bar{T}$ & $T$ & $\mathrm{G}$ & $A$ & $C$ \\
\hline $\begin{array}{c}\text { AFRIC } \\
\text { A }\end{array}$ & Yoruba & $\begin{array}{c}\text { YRB_JK3 } \\
887\end{array}$ & $\mathrm{~T}$ & $T$ & $\mathrm{G}$ & $\mathrm{C}$ & $\mathrm{G}$ & A & $\mathrm{C}$ & $\mathrm{C}$ & $A$ & $\mathrm{~T}$ & $A$ & $\mathrm{~T}$ & $T$ & $T$ & $\mathrm{G}$ & A & $\mathrm{C}$ \\
\hline $\begin{array}{c}\text { AFRIC } \\
\text { A }\end{array}$ & Yoruba & $\begin{array}{c}\text { YRB_JK3 } \\
888\end{array}$ & $\mathrm{~T}$ & $T$ & $\mathrm{G}$ & C & $\mathrm{G}$ & $\bar{A}$ & C & $\mathrm{C}$ & $A$ & $T$ & A & $\mathrm{G}$ & $T$ & $T$ & $\mathrm{G}$ & A & $C$ \\
\hline $\begin{array}{c}\text { AFRIC } \\
\text { A }\end{array}$ & Yoruba & $\begin{array}{c}\text { YRB_JK3 } \\
888\end{array}$ & $\bar{T}$ & $\bar{T}$ & $\mathrm{G}$ & $\mathrm{C}$ & $\mathrm{G}$ & $A$ & $\mathrm{C}$ & $C$ & $A$ & $T$ & $A$ & $\mathrm{~T}$ & $\mathrm{~T}$ & $T$ & $\mathrm{G}$ & $\bar{A}$ & $\mathrm{C}$ \\
\hline AFRIC & Yoruba & YRB_JK3 & $\mathrm{T}$ & $\bar{T}$ & G & $\mathrm{C}$ & $G$ & A & $C$ & $C$ & A & $\bar{T}$ & $A$ & $T$ & $T$ & $T$ & $\mathrm{G}$ & $A$ & $\mathrm{C}$ \\
\hline
\end{tabular}

John Wiley ${ }^{4}$ \& Sons, Inc. 


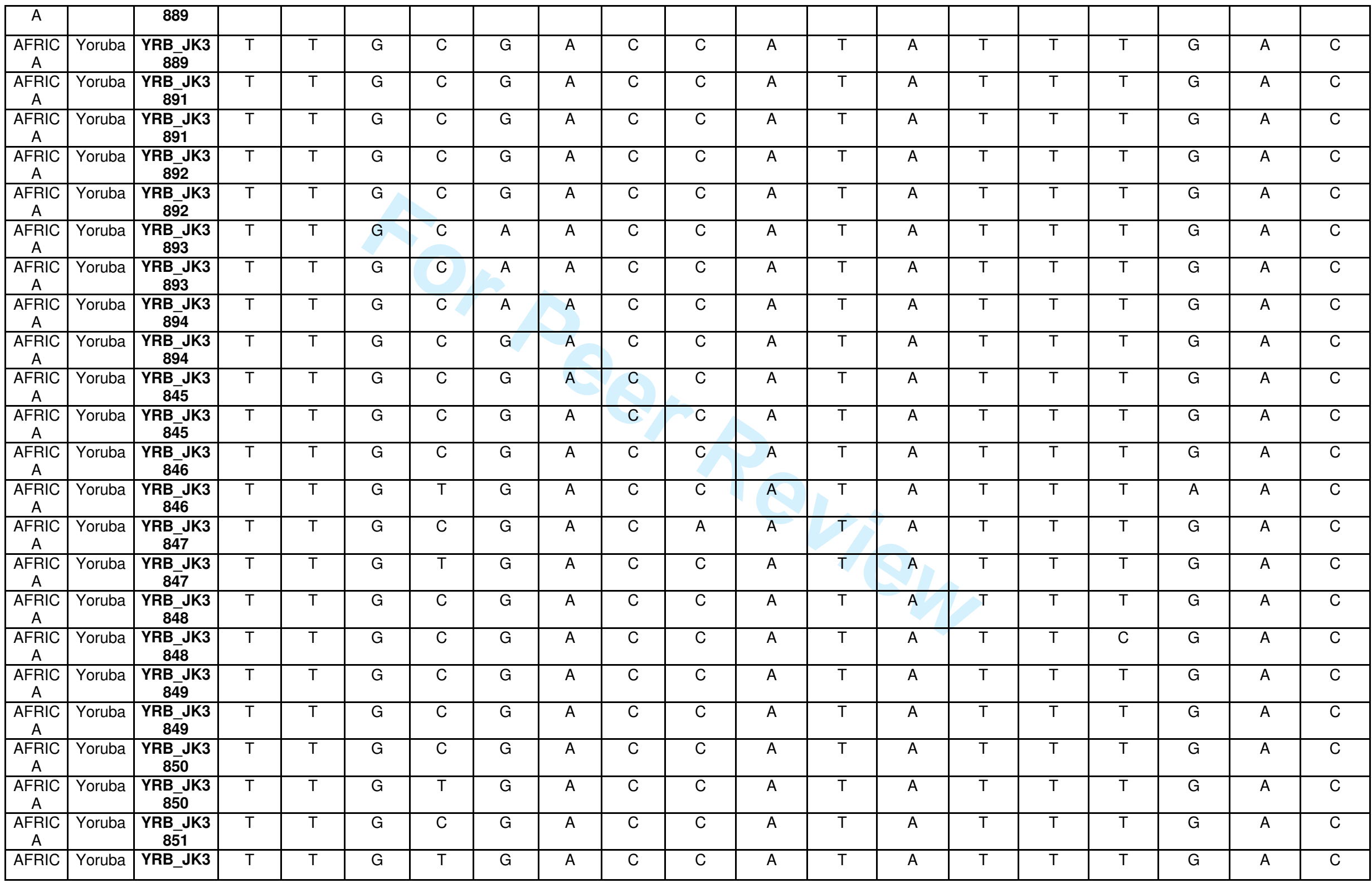

John Wiley ${ }^{5}$ Sons, Inc. 


\begin{tabular}{|c|c|c|c|c|c|c|c|c|c|c|c|c|c|c|c|c|c|c|c|}
\hline$A$ & & 851 & & & & & & & & & & & & & & & & & \\
\hline $\begin{array}{c}\text { AFRIC } \\
\text { A }\end{array}$ & Yoruba & $\begin{array}{c}\text { YRB_JK3 } \\
852\end{array}$ & $\mathrm{~T}$ & $T$ & $\mathrm{G}$ & $C$ & $\bar{G}$ & $\bar{A}$ & $\mathrm{C}$ & C & $\bar{A}$ & $T$ & $\bar{A}$ & $\mathrm{~T}$ & $T$ & $T$ & $\mathrm{G}$ & $A$ & $\mathrm{C}$ \\
\hline $\begin{array}{c}\text { AFRIC } \\
\text { A }\end{array}$ & Yoruba & $\begin{array}{c}\text { YRB_JK3 } \\
852\end{array}$ & $\mathrm{~T}$ & $T$ & $\mathrm{G}$ & $\mathrm{C}$ & $\bar{G}$ & $A$ & $\mathrm{C}$ & $C$ & $\bar{A}$ & $T$ & $\bar{A}$ & $\mathrm{~T}$ & $T$ & $T$ & $\mathrm{G}$ & $A$ & $\mathrm{C}$ \\
\hline $\begin{array}{c}\text { AFRIC } \\
\text { A }\end{array}$ & Yoruba & $\begin{array}{c}\text { YRB_JK3 } \\
853\end{array}$ & $\mathrm{~T}$ & $T$ & $\mathrm{G}$ & $\mathrm{C}$ & A & $A$ & $\mathrm{C}$ & $C$ & $A$ & $T$ & A & $\mathrm{T}$ & $\mathrm{T}$ & $T$ & $\mathrm{G}$ & $A$ & $\mathrm{C}$ \\
\hline $\begin{array}{c}\text { AFRIC } \\
\text { A }\end{array}$ & Yoruba & $\begin{array}{c}\text { YRB_JK3 } \\
853\end{array}$ & $\mathrm{~T}$ & $T$ & $\mathrm{G}$ & $\mathrm{C}$ & $\mathrm{G}$ & $T$ & $\mathrm{C}$ & $C$ & A & $T$ & A & $\mathrm{T}$ & $T$ & $T$ & $\mathrm{G}$ & $A$ & $\mathrm{C}$ \\
\hline $\begin{array}{c}\text { AFRIC } \\
\text { A }\end{array}$ & Yoruba & $\begin{array}{c}\text { YRB_JK3 } \\
854\end{array}$ & $\mathrm{~T}$ & $T$ & $\mathrm{G}$ & $\mathrm{C}$ & $\bar{G}$ & $\bar{A}$ & C & C & $\bar{A}$ & $T$ & $\bar{A}$ & $\mathrm{~T}$ & $T$ & $T$ & $\mathrm{G}$ & $A$ & $\mathrm{C}$ \\
\hline $\begin{array}{c}\text { AFRIC } \\
\text { A }\end{array}$ & Yoruba & $\begin{array}{c}\text { YRB_JK3 } \\
854\end{array}$ & $\mathrm{~T}$ & $T$ & $\mathrm{G}$ & $\mathrm{T}$ & $\mathrm{G}$ & A & $\mathrm{C}$ & $C$ & A & $T$ & A & $\mathrm{T}$ & $\mathrm{T}$ & $\bar{T}$ & $\mathrm{G}$ & $A$ & $\mathrm{C}$ \\
\hline $\begin{array}{c}\text { AFRIC } \\
\text { A }\end{array}$ & Yoruba & $\begin{array}{c}\text { YRB_JK3 } \\
855\end{array}$ & $\mathrm{~T}$ & $T$ & $\mathrm{G}$ & $\mathrm{C}$ & A & A & $\mathrm{C}$ & $C$ & A & $T$ & A & $\mathrm{T}$ & $T$ & $T$ & $\mathrm{G}$ & $A$ & $\mathrm{C}$ \\
\hline $\begin{array}{c}\text { AFRIC } \\
\text { A }\end{array}$ & Yoruba & $\begin{array}{c}\text { YRB_JK3 } \\
855\end{array}$ & $\mathrm{~T}$ & $T$ & $\mathrm{G}$ & $C$ & $G$ & $A$ & $\mathrm{C}$ & C & A & $T$ & A & $\mathrm{T}$ & $T$ & $T$ & $\mathrm{G}$ & $A$ & $\mathrm{C}$ \\
\hline $\begin{array}{c}\text { AFRIC } \\
\text { A }\end{array}$ & Yoruba & $\begin{array}{c}\text { YRB_JK3 } \\
856\end{array}$ & $\mathrm{~T}$ & $\mathrm{~T}$ & $\mathrm{G}$ & C & $G$ & A & C & C & A & $\mathrm{T}$ & A & $\mathrm{T}$ & $\mathrm{T}$ & $\mathrm{T}$ & $\mathrm{G}$ & A & $\mathrm{T}$ \\
\hline $\begin{array}{c}\text { AFRIC } \\
\text { A }\end{array}$ & Yoruba & $\begin{array}{c}\text { YRB_JK3 } \\
856\end{array}$ & $\mathrm{~T}$ & $\mathrm{~T}$ & $G$ & $\mathrm{~T}$ & $\mathrm{G}$ & A & $C$ & C & A & $\mathrm{T}$ & A & $\mathrm{T}$ & $\mathrm{T}$ & $T$ & $G$ & $A$ & C \\
\hline $\begin{array}{c}\text { AFRIC } \\
\text { A }\end{array}$ & Yoruba & $\begin{array}{c}\text { YRB_JK3 } \\
860\end{array}$ & $\mathrm{~T}$ & $T$ & $\mathrm{G}$ & $\mathrm{C}$ & $\bar{A}$ & $\bar{A}$ & $\mathrm{C}$ & $\mathrm{C}$ & A & $T$ & $\bar{A}$ & $\mathrm{~T}$ & $T$ & $T$ & $\mathrm{G}$ & $A$ & $\mathrm{C}$ \\
\hline $\begin{array}{c}\text { AFRIC } \\
\text { A }\end{array}$ & Yoruba & $\begin{array}{c}\text { YRB_JK3 } \\
860\end{array}$ & $\mathrm{~T}$ & $\mathrm{~T}$ & $\mathrm{G}$ & C & G & A & C & C & A & $\mathrm{T}$ & A & $\mathrm{T}$ & $\mathrm{T}$ & $\mathrm{T}$ & $G$ & A & $\mathrm{C}$ \\
\hline $\begin{array}{c}\text { AFRIC } \\
\text { A }\end{array}$ & Yoruba & $\begin{array}{c}\text { YRB_JK3 } \\
858 \\
\end{array}$ & $T$ & $\mathrm{~T}$ & $\mathrm{G}$ & $C$ & $\mathrm{G}$ & $\bar{A}$ & $\mathrm{C}$ & $C$ & $A$ & $\mathrm{~T}$ & $A$ & $T$ & $\bar{T}$ & $\bar{T}$ & $\mathrm{G}$ & $A$ & $\mathrm{C}$ \\
\hline $\begin{array}{c}\text { AFRIC } \\
\text { A }\end{array}$ & Yoruba & $\begin{array}{c}\text { YRB_JK3 } \\
858\end{array}$ & $\mathrm{~T}$ & $T$ & $\mathrm{G}$ & $\mathrm{T}$ & $\bar{G}$ & $\mathrm{~T}$ & $\mathrm{C}$ & C & $\bar{A}$ & $T$ & $\bar{A}$ & $\mathrm{~T}$ & $\mathrm{~T}$ & $\mathrm{~T}$ & $\mathrm{G}$ & $A$ & $\mathrm{C}$ \\
\hline $\begin{array}{c}\text { AFRIC } \\
\text { A }\end{array}$ & Yoruba & $\begin{array}{c}\text { YRB_JK3 } \\
859\end{array}$ & $\mathrm{~T}$ & $\mathrm{~T}$ & $G$ & $C$ & $G$ & A & $C$ & $C$ & A & $T$ & A & $T$ & $\mathrm{~T}$ & $\mathrm{~T}$ & $\mathrm{G}$ & $A$ & $\mathrm{C}$ \\
\hline $\begin{array}{c}\text { AFRIC } \\
\text { A }\end{array}$ & Yoruba & $\begin{array}{c}\text { YRB_JK3 } \\
859 \\
\end{array}$ & $\mathrm{~T}$ & $\mathrm{~T}$ & $\mathrm{G}$ & $C$ & $\mathrm{G}$ & $A$ & $\mathrm{C}$ & $C$ & $A$ & $\mathrm{~T}$ & $A$ & $T$ & $\bar{T}$ & $\bar{T}$ & $\mathrm{G}$ & $A$ & $\mathrm{C}$ \\
\hline $\begin{array}{l}\text { East- } \\
\text { Asia }\end{array}$ & China & $\underset{5}{\mathrm{CH}}$ & $\mathrm{T}$ & $T$ & $\mathrm{G}$ & $\mathrm{C}$ & $\bar{A}$ & A & $\mathrm{C}$ & C & A & $T$ & A & $T$ & $\bar{T}$ & $T$ & $\mathrm{G}$ & $A$ & $\mathrm{C}$ \\
\hline $\begin{array}{l}\text { East- } \\
\text { Asia }\end{array}$ & China & $\underset{5}{\mathrm{CH}}$ & $\mathrm{T}$ & $T$ & $\mathrm{G}$ & $\mathrm{C}$ & $G$ & A & C & C & A & $T$ & A & $T$ & $\mathrm{~T}$ & $\mathrm{~T}$ & $\mathrm{G}$ & $A$ & $\mathrm{C}$ \\
\hline $\begin{array}{l}\text { East- } \\
\text { Asia }\end{array}$ & China & $\begin{array}{c}\mathrm{CH}+\mathrm{CHO} \\
2\end{array}$ & $\mathrm{~T}$ & $\mathrm{~T}$ & $\mathrm{G}$ & $\mathrm{C}$ & $A$ & A & $\mathrm{C}$ & $C$ & A & $T$ & A & $T$ & $T$ & $T$ & $\mathrm{G}$ & $A$ & $\mathrm{C}$ \\
\hline $\begin{array}{l}\text { East- } \\
\text { Asia }\end{array}$ & China & $\underset{2}{\mathrm{CH}} \mathrm{CHO}$ & $\mathrm{C}$ & $T$ & $\mathrm{G}$ & $\mathrm{C}$ & $G$ & A & C & C & A & $T$ & $\bar{A}$ & $T$ & $\mathrm{~T}$ & $T$ & $\mathrm{G}$ & $\mathrm{A}$ & $\mathrm{C}$ \\
\hline $\begin{array}{l}\text { East- } \\
\text { Asia } \\
\end{array}$ & China & $\underset{3}{\mathrm{CH}}$ & $\mathrm{T}$ & $T$ & $\mathrm{G}$ & $\mathrm{C}$ & A & A & C & C & A & $T$ & A & $T$ & $\mathrm{~T}$ & $\bar{T}$ & $\mathrm{G}$ & $A$ & $\mathrm{C}$ \\
\hline $\begin{array}{l}\text { East- } \\
\text { Asia }\end{array}$ & China & $\underset{3}{\mathrm{CH}}$ & $\mathrm{T}$ & $T$ & $\mathrm{G}$ & $\mathrm{C}$ & $\bar{G}$ & $A$ & $\mathrm{C}$ & $C$ & $A$ & $T$ & $\bar{A}$ & $T$ & $\bar{T}$ & $\bar{T}$ & $\mathrm{G}$ & $A$ & $\mathrm{C}$ \\
\hline East- & China & $\mathrm{CH} \_\mathrm{CHO}$ & $\mathrm{T}$ & $T$ & $\mathrm{G}$ & $\mathrm{C}$ & A & A & $\mathrm{C}$ & C & A & $T$ & A & $T$ & $\mathrm{~T}$ & $\mathrm{~T}$ & $\mathrm{G}$ & $\mathrm{A}$ & $\mathrm{C}$ \\
\hline
\end{tabular}

John Wiley ${ }^{6}$ Sons, Inc. 


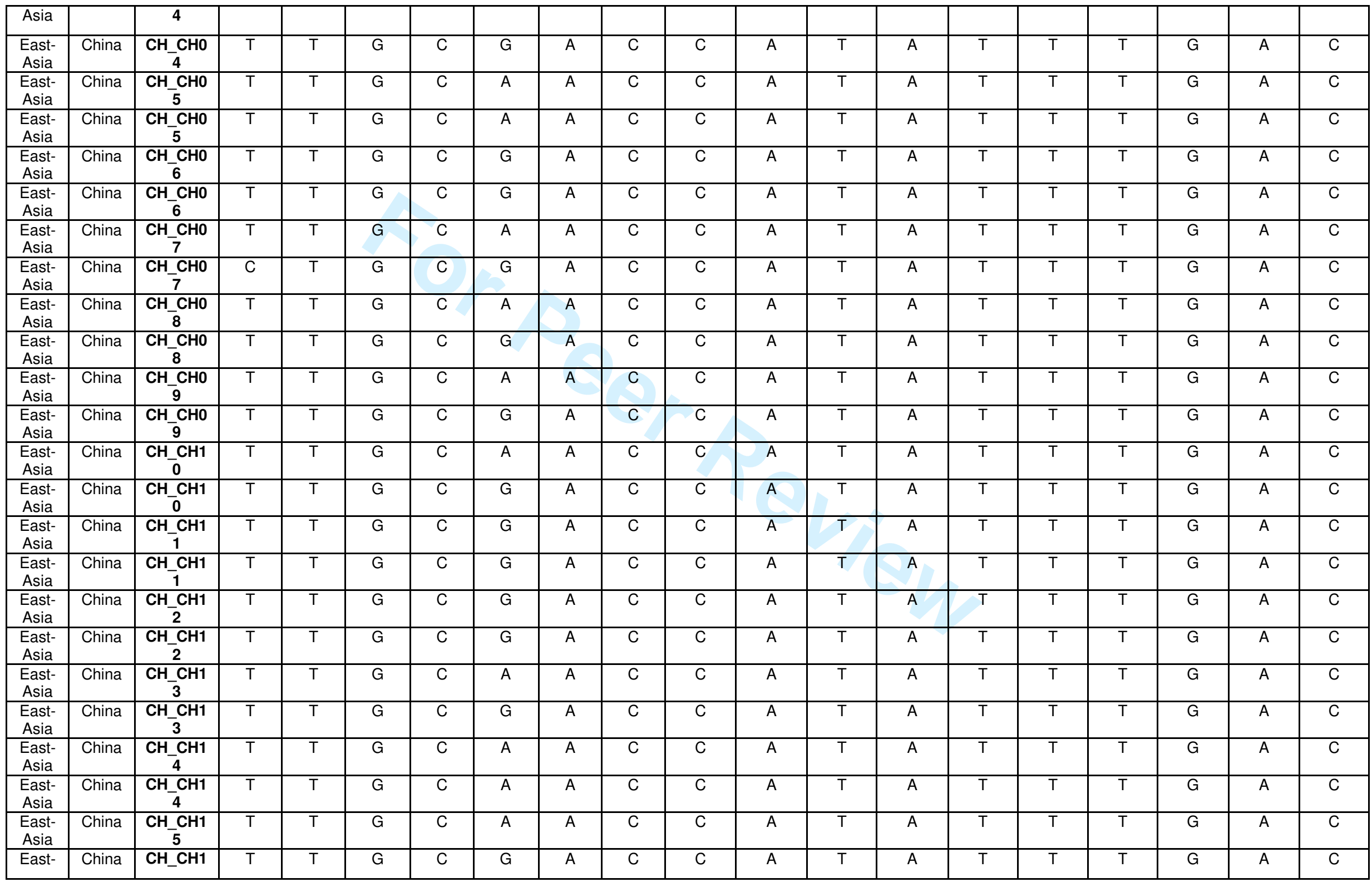

John Wiley ${ }^{7}$ \& Sons, Inc. 


\begin{tabular}{|c|c|c|c|c|c|c|c|c|c|c|c|c|c|c|c|c|c|c|c|}
\hline Asia & & 5 & & & & & & & & & & & & & & & & & \\
\hline $\begin{array}{l}\text { East- } \\
\text { Asia }\end{array}$ & China & $\underset{6}{\mathrm{CH} \_\mathrm{CH} 1}$ & $\mathrm{~T}$ & $T$ & $\mathrm{G}$ & $\mathrm{C}$ & $\bar{A}$ & $\bar{A}$ & C & C & $A$ & $\mathrm{~T}$ & $A$ & $\mathrm{~T}$ & $\mathrm{~T}$ & $\mathrm{~T}$ & $\mathrm{G}$ & $A$ & $\mathrm{C}$ \\
\hline $\begin{array}{l}\text { East- } \\
\text { Asia }\end{array}$ & China & $\mathrm{CH}+6 \mathrm{CH} 1$ & $\mathrm{~T}$ & $T$ & $\mathrm{G}$ & $\mathrm{C}$ & A & A & $\mathrm{C}$ & C & $A$ & $\mathrm{~T}$ & $A$ & $\mathrm{~T}$ & $T$ & $T$ & $\mathrm{G}$ & $A$ & C \\
\hline $\begin{array}{l}\text { East- } \\
\text { Asia }\end{array}$ & China & CH_CH1 & $\mathrm{T}$ & $T$ & $\mathrm{G}$ & $\mathrm{C}$ & $A$ & $\bar{A}$ & $C$ & C & $\mathrm{A}$ & $T$ & $A$ & $\mathrm{~T}$ & $\mathrm{~T}$ & $\bar{T}$ & $G$ & $\mathrm{~A}$ & $\mathrm{C}$ \\
\hline $\begin{array}{l}\text { East- } \\
\text { Asia }\end{array}$ & China & $\underset{7}{\mathrm{CH}+\mathrm{CH} 1}$ & $\mathrm{~T}$ & $\mathrm{~T}$ & $\mathrm{G}$ & $C$ & $\mathrm{G}$ & $A$ & C & C & $A$ & $T$ & $A$ & $\mathrm{~T}$ & $\mathrm{~T}$ & $T$ & $\mathrm{G}$ & $A$ & C \\
\hline $\begin{array}{l}\text { East- } \\
\text { Asia }\end{array}$ & China & $\underset{8}{\mathrm{CH} C \mathrm{CH} 1}$ & $\mathrm{~T}$ & $T$ & $\mathrm{G}$ & C & $\mathrm{G}$ & A & C & $C$ & $A$ & $\mathrm{~T}$ & $A$ & $\mathrm{~T}$ & $T$ & $T$ & $G$ & $A$ & C \\
\hline $\begin{array}{l}\text { East- } \\
\text { Asia }\end{array}$ & China & $\underset{8}{\mathrm{CH}} \mathrm{CH} 1$ & $\mathrm{~T}$ & $T$ & $\mathrm{G}$ & $\mathrm{C}$ & $G$ & A & C & C & A & $\mathrm{T}$ & $A$ & $\mathrm{~T}$ & $T$ & $T$ & $G$ & $A$ & C \\
\hline $\begin{array}{l}\text { East- } \\
\text { Asia }\end{array}$ & China & $\underset{9}{\mathrm{CH} C \mathrm{CH} 1}$ & $\mathrm{~T}$ & $\mathrm{~T}$ & $\mathrm{G}$ & $C$ & $A$ & $A$ & $C$ & $C$ & $A$ & $T$ & $A$ & $\mathrm{~T}$ & $\mathrm{G}$ & $T$ & $\mathrm{G}$ & $A$ & $\mathrm{C}$ \\
\hline $\begin{array}{l}\text { East- } \\
\text { Asia }\end{array}$ & China & $\underset{9}{\mathrm{CH}} \mathrm{CH} 1$ & $\mathrm{~T}$ & $T$ & $G$ & $\mathrm{C}$ & A & $A$ & C & C & A & $\mathrm{T}$ & $A$ & $\mathrm{~T}$ & $T$ & $T$ & $\mathrm{G}$ & $A$ & C \\
\hline $\begin{array}{l}\text { East- } \\
\text { Asia }\end{array}$ & China & $\underset{0}{\mathrm{CH} C \mathrm{CH} 2}$ & $\mathrm{~T}$ & $T$ & $G$ & $\mathrm{C}$ & $A$ & A & C & C & A & $\mathrm{T}$ & $A$ & $\mathrm{~T}$ & $T$ & $T$ & $\mathrm{G}$ & $A$ & C \\
\hline $\begin{array}{l}\text { East- } \\
\text { Asia }\end{array}$ & China & $\underset{0}{\mathrm{CH} C \mathrm{CH} 2}$ & $\mathrm{~T}$ & $T$ & $\mathrm{G}$ & $C$ & $\mathrm{G}$ & $A$ & C & $C$ & $A$ & $T$ & $A$ & $\mathrm{~T}$ & $T$ & $T$ & $\mathrm{G}$ & $\mathrm{A}$ & $\mathrm{C}$ \\
\hline $\begin{array}{l}\text { East- } \\
\text { Asia }\end{array}$ & China & $\underset{1}{\mathrm{CH}}$ & $\mathrm{T}$ & $T$ & $\mathrm{G}$ & $\mathrm{C}$ & $A$ & $A$ & C & C & $A$ & $\mathrm{~T}$ & $A$ & $T$ & $T$ & $T$ & $\mathrm{G}$ & $A$ & C \\
\hline $\begin{array}{l}\text { East- } \\
\text { Asia }\end{array}$ & China & $\mathrm{CH} \underset{1}{\mathrm{CH} 2}$ & $\mathrm{~T}$ & $T$ & $\mathrm{G}$ & $\mathrm{C}$ & $\mathrm{G}$ & A & $\mathrm{C}$ & C & A & $\mathrm{T}$ & $A$ & $\mathrm{~T}$ & $T$ & $T$ & $\mathrm{G}$ & $A$ & C \\
\hline $\begin{array}{l}\text { East- } \\
\text { Asia }\end{array}$ & China & $\underset{2}{\mathrm{CH} C \mathrm{CH} 2}$ & $\mathrm{~T}$ & $T$ & $\bar{G}$ & $\mathrm{C}$ & $\bar{G}$ & $\bar{A}$ & $\mathrm{C}$ & C & $\bar{A}$ & $T$ & $A$ & $T$ & $\mathrm{~T}$ & $T$ & $\mathrm{G}$ & $\mathrm{A}$ & $\mathrm{C}$ \\
\hline $\begin{array}{l}\text { East- } \\
\text { Asia }\end{array}$ & China & $\underset{2}{\mathrm{CH} \_\mathrm{CH} 2}$ & $C$ & $T$ & $\mathrm{G}$ & $\mathrm{C}$ & $\mathrm{G}$ & $\bar{A}$ & $\mathrm{C}$ & C & $A$ & $\mathrm{~T}$ & $A$ & $T$ & $\mathrm{~T}$ & $T$ & $\mathrm{G}$ & $A$ & $\mathrm{C}$ \\
\hline $\begin{array}{l}\text { East- } \\
\text { Asia }\end{array}$ & China & $\mathrm{CH}_{3} \mathrm{CH} 2$ & $\mathrm{~T}$ & $T$ & $\mathrm{G}$ & $\mathrm{C}$ & $\bar{A}$ & A & C & $C$ & A & $\mathrm{T}$ & $A$ & $\mathrm{~T}$ & $\mathrm{~T}$ & $\mathrm{~T}$ & $G$ & $A$ & C \\
\hline $\begin{array}{l}\text { East- } \\
\text { Asia }\end{array}$ & China & $\underset{3}{\mathrm{CH} C \mathrm{CH} 2}$ & $\mathrm{~T}$ & $T$ & $\mathrm{G}$ & $\mathrm{C}$ & $\bar{G}$ & $\bar{A}$ & $\mathrm{C}$ & C & $\bar{A}$ & $T$ & $A$ & $T$ & $\bar{T}$ & $T$ & $\bar{G}$ & $A$ & $\mathrm{C}$ \\
\hline $\begin{array}{l}\text { East- } \\
\text { Asia }\end{array}$ & China & $\underset{4}{\mathrm{CH}} \mathrm{CH} 2$ & $\mathrm{~T}$ & $T$ & $\mathrm{G}$ & $\mathrm{C}$ & $A$ & A & $\mathrm{C}$ & C & A & $\mathrm{T}$ & $A$ & $T$ & $\mathrm{G}$ & $T$ & $\mathrm{G}$ & $A$ & $\mathrm{C}$ \\
\hline $\begin{array}{l}\text { East- } \\
\text { Asia }\end{array}$ & China & $\mathrm{CH}_{4} \mathrm{CH} 2$ & $\mathrm{~T}$ & $T$ & $G$ & $\mathrm{C}$ & $A$ & A & C & $C$ & A & $\mathrm{T}$ & $A$ & $T$ & $\mathrm{~T}$ & $\mathrm{~T}$ & $\mathrm{G}$ & $A$ & C \\
\hline $\begin{array}{l}\text { East- } \\
\text { Asia }\end{array}$ & $\begin{array}{c}\text { Japane } \\
\text { se }\end{array}$ & JPN_J36 & $\mathrm{T}$ & $T$ & $\mathrm{G}$ & $\mathrm{C}$ & $G$ & A & $\mathrm{C}$ & C & A & $\mathrm{T}$ & $A$ & $\mathrm{~T}$ & $\mathrm{~T}$ & $T$ & $\mathrm{G}$ & $A$ & $\mathrm{C}$ \\
\hline $\begin{array}{l}\text { East- } \\
\text { Asia }\end{array}$ & $\begin{array}{c}\text { Japane } \\
\text { se }\end{array}$ & JPN_J36 & $\mathrm{T}$ & $T$ & $\mathrm{G}$ & $\mathrm{C}$ & $G$ & A & $\mathrm{C}$ & C & A & $\mathrm{T}$ & $\mathrm{A}$ & $\mathrm{T}$ & $\mathrm{T}$ & $T$ & $\mathrm{G}$ & $A$ & $\mathrm{C}$ \\
\hline $\begin{array}{l}\text { East- } \\
\text { Asia }\end{array}$ & $\begin{array}{c}\text { Japane } \\
\text { se }\end{array}$ & JPN_J37 & $\mathrm{T}$ & $T$ & $\mathrm{G}$ & $\mathrm{C}$ & A & A & C & C & $\mathrm{G}$ & $\mathrm{T}$ & $A$ & $\mathrm{~T}$ & $\mathrm{~T}$ & $\mathrm{~T}$ & $\mathrm{G}$ & $A$ & $\mathrm{C}$ \\
\hline $\begin{array}{l}\text { East- } \\
\text { Asia }\end{array}$ & $\begin{array}{c}\text { Japane } \\
\text { se }\end{array}$ & JPN_J37 & $\mathrm{T}$ & $T$ & $G$ & $\mathrm{C}$ & $G$ & $\bar{A}$ & $\mathrm{C}$ & $C$ & $\bar{A}$ & $\mathrm{~T}$ & $A$ & $\mathrm{~T}$ & $\mathrm{~T}$ & $T$ & $G$ & $A$ & $\mathrm{C}$ \\
\hline East- & Japane & JPN_J38 & $\mathrm{T}$ & $T$ & $\mathrm{G}$ & $\mathrm{C}$ & A & A & $\mathrm{C}$ & C & A & $\mathrm{T}$ & A & $\mathrm{T}$ & $\mathrm{T}$ & $T$ & $\mathrm{G}$ & $\mathrm{A}$ & $\mathrm{C}$ \\
\hline
\end{tabular}

John Wiley \& Sons, Inc. 


\begin{tabular}{|c|c|c|c|c|c|c|c|c|c|c|c|c|c|c|c|c|c|c|c|}
\hline Asia & se & & & & & & & & & & & & & & & & & & \\
\hline $\begin{array}{l}\text { East- } \\
\text { Asia } \\
\end{array}$ & $\begin{array}{c}\text { Japane } \\
\text { se }\end{array}$ & JPN_J38 & $\mathrm{T}$ & $T$ & $\mathrm{G}$ & $\mathrm{C}$ & $A$ & $A$ & $\mathrm{C}$ & $\mathrm{C}$ & $A$ & $T$ & A & $\mathrm{T}$ & $T$ & $T$ & $\bar{G}$ & $\bar{A}$ & C \\
\hline $\begin{array}{l}\text { East- } \\
\text { Asia } \\
\end{array}$ & $\begin{array}{c}\text { Japane } \\
\text { se }\end{array}$ & JPN_J39 & $\mathrm{T}$ & $T$ & $\mathrm{G}$ & $\mathrm{C}$ & $A$ & $A$ & $\mathrm{C}$ & $\mathrm{C}$ & $A$ & $T$ & $A$ & $\bar{T}$ & $\bar{T}$ & $\bar{T}$ & $\mathrm{G}$ & $\bar{A}$ & C \\
\hline $\begin{array}{l}\text { East- } \\
\text { Asia }\end{array}$ & $\begin{array}{c}\text { Japane } \\
\text { se }\end{array}$ & JPN_J39 & $\mathrm{T}$ & $T$ & $\mathrm{G}$ & $\mathrm{C}$ & $\mathrm{G}$ & $\mathrm{A}$ & $\mathrm{C}$ & $C$ & $A$ & $T$ & A & $\mathrm{T}$ & $T$ & $\mathrm{~T}$ & $\bar{G}$ & $A$ & $C$ \\
\hline $\begin{array}{l}\text { East- } \\
\text { Asia }\end{array}$ & $\begin{array}{c}\text { Japane } \\
\text { se }\end{array}$ & JPN_J40 & $\mathrm{T}$ & $T$ & $\mathrm{G}$ & $\mathrm{C}$ & $A$ & $A$ & $\mathrm{C}$ & $C$ & $A$ & $T$ & A & $\mathrm{T}$ & $\mathrm{T}$ & $T$ & $\mathrm{G}$ & $A$ & $C$ \\
\hline $\begin{array}{l}\text { East- } \\
\text { Asia }\end{array}$ & $\begin{array}{c}\text { Japane } \\
\text { se }\end{array}$ & JPN_J40 & $\mathrm{T}$ & $T$ & $\bar{G}$ & $\mathrm{C}$ & $\mathrm{G}$ & $\mathrm{A}$ & $\mathrm{C}$ & $C$ & $\mathrm{~A}$ & $T$ & $A$ & $\mathrm{~T}$ & $T$ & $T$ & $\mathrm{G}$ & $\bar{A}$ & C \\
\hline $\begin{array}{l}\text { East- } \\
\text { Asia }\end{array}$ & $\begin{array}{c}\text { Japane } \\
\text { se }\end{array}$ & JPN_J41 & $\mathrm{T}$ & $T$ & $\mathrm{G}$ & $\mathrm{C}$ & $A$ & $A$ & $\mathrm{C}$ & $C$ & $A$ & $\mathrm{~T}$ & A & $\mathrm{T}$ & $\mathrm{T}$ & $\mathrm{T}$ & $\mathrm{G}$ & $A$ & $C$ \\
\hline $\begin{array}{l}\text { East- } \\
\text { Asia }\end{array}$ & $\begin{array}{c}\text { Japane } \\
\text { se }\end{array}$ & JPN_J41 & $\mathrm{T}$ & $T$ & $\mathrm{G}$ & $\mathrm{C}$ & $A$ & $A$ & $\mathrm{C}$ & $C$ & $A$ & $T$ & A & $\mathrm{T}$ & $T$ & $T$ & $G$ & $A$ & $C$ \\
\hline $\begin{array}{l}\text { East- } \\
\text { Asia }\end{array}$ & $\begin{array}{c}\text { Japane } \\
\text { se }\end{array}$ & JPN_J42 & $\mathrm{T}$ & $T$ & $\mathrm{G}$ & $\mathrm{C}$ & $\mathrm{A}$ & $\mathrm{A}$ & $\mathrm{C}$ & $C$ & $\mathrm{~A}$ & $\mathrm{~T}$ & $A$ & $\mathrm{~T}$ & $\mathrm{~T}$ & $T$ & $\mathrm{G}$ & $A$ & $C$ \\
\hline $\begin{array}{l}\text { East- } \\
\text { Asia }\end{array}$ & $\begin{array}{c}\text { Japane } \\
\text { se }\end{array}$ & JPN_J42 & $\mathrm{T}$ & $T$ & $\mathrm{G}$ & $\mathrm{C}$ & $\mathrm{G}$ & $A$ & $\mathrm{C}$ & $C$ & $A$ & $\mathrm{~T}$ & $A$ & $T$ & $\mathrm{~T}$ & $\mathrm{~T}$ & $\mathrm{G}$ & $A$ & $C$ \\
\hline $\begin{array}{l}\text { East- } \\
\text { Asia }\end{array}$ & $\begin{array}{c}\text { Japane } \\
\text { se }\end{array}$ & $\begin{array}{c}\text { JPN_JK1 } \\
4 \mathbf{2 4}\end{array}$ & $\mathrm{T}$ & $T$ & $\mathrm{G}$ & $\mathrm{C}$ & $\mathrm{G}$ & $A$ & C & $C$ & $A$ & $T$ & A & $\mathrm{T}$ & $\mathrm{T}$ & $T$ & $G$ & $A$ & C \\
\hline $\begin{array}{l}\text { East- } \\
\text { Asia }\end{array}$ & $\begin{array}{c}\text { Japane } \\
\text { se }\end{array}$ & $\begin{array}{c}\text { JPN_JK1 } \\
424\end{array}$ & $\mathrm{~T}$ & $\mathrm{~T}$ & $\bar{G}$ & $\mathrm{C}$ & $\mathrm{G}$ & $\bar{A}$ & $\mathrm{C}$ & $C$ & $\mathrm{~A}$ & $T$ & $\mathrm{~A}$ & $\mathrm{~T}$ & $T$ & $T$ & $\mathrm{G}$ & $\mathrm{A}$ & C \\
\hline $\begin{array}{l}\text { East- } \\
\text { Asia }\end{array}$ & $\begin{array}{c}\text { Japane } \\
\text { se }\end{array}$ & $\begin{array}{c}\text { JPN_JK1 } \\
425\end{array}$ & $\mathrm{~T}$ & $T$ & $\mathrm{G}$ & $\mathrm{C}$ & $\mathrm{G}$ & $A$ & $\mathrm{C}$ & $C$ & $A$ & $T$ & $A$ & $T$ & $T$ & $T$ & $\mathrm{G}$ & $A$ & C \\
\hline $\begin{array}{l}\text { East- } \\
\text { Asia }\end{array}$ & $\begin{array}{c}\text { Japane } \\
\text { se }\end{array}$ & $\underset{425}{\text { JPN_JK1 }}$ & $\mathrm{T}$ & $T$ & $\mathrm{G}$ & $\mathrm{C}$ & $\mathrm{G}$ & $\mathrm{A}$ & $\mathrm{C}$ & $C$ & $A$ & $T$ & A & $T$ & $\mathrm{~T}$ & $T$ & $\mathrm{G}$ & $A$ & $C$ \\
\hline $\begin{array}{l}\text { East- } \\
\text { Asia }\end{array}$ & $\begin{array}{c}\text { Japane } \\
\text { se }\end{array}$ & $\underset{426}{\text { JPN_JK1 }}$ & $\mathrm{T}$ & $T$ & $\bar{G}$ & $\mathrm{C}$ & $\mathrm{G}$ & $\bar{A}$ & $\mathrm{C}$ & $C$ & $\mathrm{~A}$ & $T$ & $A$ & $T$ & $T$ & $T$ & $\mathrm{G}$ & $A$ & C \\
\hline $\begin{array}{l}\text { East- } \\
\text { Asia }\end{array}$ & $\begin{array}{c}\text { Japane } \\
\text { se }\end{array}$ & $\begin{array}{c}\text { JPN_JK1 } \\
426\end{array}$ & $\mathrm{~T}$ & $T$ & $\mathrm{G}$ & $\mathrm{C}$ & $\mathrm{G}$ & $A$ & $\mathrm{C}$ & $C$ & $A$ & $T$ & $A$ & $T$ & $T$ & $T$ & $\bar{G}$ & $A$ & $C$ \\
\hline $\begin{array}{l}\text { East- } \\
\text { Asia }\end{array}$ & $\begin{array}{c}\text { Japane } \\
\text { se }\end{array}$ & JPN_J30 & $\mathrm{T}$ & $T$ & $\mathrm{G}$ & $\mathrm{C}$ & $\mathrm{G}$ & $\mathrm{A}$ & $\mathrm{C}$ & $C$ & $A$ & $T$ & $A$ & $T$ & $T$ & $T$ & $\mathrm{G}$ & $A$ & $C$ \\
\hline $\begin{array}{l}\text { East- } \\
\text { Asia }\end{array}$ & $\begin{array}{c}\text { Japane } \\
\text { se }\end{array}$ & JPN_J30 & $\mathrm{T}$ & $T$ & $G$ & $\mathrm{C}$ & $\mathrm{G}$ & $\mathrm{A}$ & $\mathrm{C}$ & C & $A$ & $T$ & $A$ & $T$ & $T$ & $T$ & $\mathrm{G}$ & $A$ & $C$ \\
\hline $\begin{array}{l}\text { East- } \\
\text { Asia }\end{array}$ & $\begin{array}{c}\text { Japane } \\
\text { se }\end{array}$ & $\begin{array}{c}\text { JPN_JK1 } \\
785\end{array}$ & $\mathrm{~T}$ & $T$ & $\mathrm{G}$ & $\mathrm{C}$ & $\mathrm{A}$ & $\mathrm{A}$ & $\mathrm{C}$ & C & $\mathrm{A}$ & $T$ & $A$ & $T$ & $T$ & $T$ & $G$ & $\bar{A}$ & C \\
\hline $\begin{array}{l}\text { East- } \\
\text { Asia }\end{array}$ & $\begin{array}{c}\text { Japane } \\
\text { se }\end{array}$ & $\begin{array}{c}\text { JPN_JK1 } \\
785\end{array}$ & $\mathrm{~T}$ & $T$ & $\mathrm{G}$ & $\mathrm{C}$ & $\mathrm{G}$ & $A$ & $\mathrm{C}$ & C & $A$ & $\mathrm{~T}$ & $A$ & $\mathrm{~T}$ & $\mathrm{~T}$ & $\mathrm{~T}$ & $\mathrm{G}$ & $A$ & C \\
\hline $\begin{array}{l}\text { East- } \\
\text { Asia }\end{array}$ & $\begin{array}{c}\text { Japane } \\
\text { se }\end{array}$ & $\begin{array}{c}\text { JPN_JK1 } \\
788\end{array}$ & $\mathrm{~T}$ & $T$ & $\mathrm{G}$ & $\mathrm{C}$ & $A$ & $A$ & $\mathrm{C}$ & $\mathrm{C}$ & $A$ & $T$ & $\bar{A}$ & $T$ & $T$ & $T$ & $G$ & $\bar{A}$ & C \\
\hline $\begin{array}{l}\text { East- } \\
\text { Asia }\end{array}$ & $\begin{array}{c}\text { Japane } \\
\text { se }\end{array}$ & $\begin{array}{c}\text { JPN_JK1 } \\
788\end{array}$ & $\mathrm{~T}$ & $T$ & $\mathrm{G}$ & $\mathrm{C}$ & $\mathrm{G}$ & $A$ & $\mathrm{C}$ & $C$ & $A$ & $T$ & $\bar{A}$ & $T$ & $T$ & $T$ & $G$ & $\bar{A}$ & C \\
\hline $\begin{array}{l}\text { East- } \\
\text { Asia }\end{array}$ & $\begin{array}{c}\text { Japane } \\
\text { se }\end{array}$ & $\begin{array}{c}\text { JPN_JK1 } \\
790\end{array}$ & $\mathrm{~T}$ & $T$ & $\mathrm{G}$ & $\mathrm{C}$ & $\mathrm{G}$ & $A$ & $\mathrm{C}$ & $C$ & $A$ & $T$ & $A$ & $T$ & $T$ & $T$ & $G$ & $\mathrm{~A}$ & C \\
\hline East- & Japane & JPN_JK1 & $\mathrm{T}$ & $T$ & $\mathrm{G}$ & $\mathrm{C}$ & $\mathrm{G}$ & $A$ & $\mathrm{C}$ & C & $A$ & $T$ & A & $\mathrm{T}$ & $T$ & $T$ & $G$ & $\bar{A}$ & C \\
\hline
\end{tabular}

John Wiley ${ }^{9}$ \& Sons, Inc. 


\begin{tabular}{|c|c|c|c|c|c|c|c|c|c|c|c|c|c|c|c|c|c|c|c|}
\hline Asia & $\mathrm{se}$ & 790 & & & & & & & & & & & & & & & & & \\
\hline $\begin{array}{l}\text { East- } \\
\text { Asia }\end{array}$ & $\begin{array}{c}\text { Japane } \\
\text { se }\end{array}$ & \begin{tabular}{|c|} 
JPN_JK1 \\
793
\end{tabular} & $\mathrm{~T}$ & $\mathrm{~T}$ & $\mathrm{G}$ & 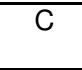 & $\mathrm{G}$ & $\bar{A}$ & $\mathrm{C}$ & $\mathrm{C}$ & $A$ & $\bar{T}$ & $\bar{A}$ & $\bar{T}$ & $\bar{T}$ & $\bar{T}$ & $\bar{G}$ & $\bar{A}$ & $\mathrm{C}$ \\
\hline $\begin{array}{l}\text { East- } \\
\text { Asia }\end{array}$ & $\begin{array}{c}\text { Japane } \\
\text { se }\end{array}$ & $\begin{array}{c}\text { JPN_JK1 } \\
793\end{array}$ & $\mathrm{C}$ & $\mathrm{T}$ & $\bar{G}$ & 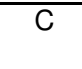 & $\bar{G}$ & $A$ & $\mathrm{C}$ & $\mathrm{C}$ & $A$ & $\bar{T}$ & $\bar{A}$ & $\bar{T}$ & $\bar{T}$ & $\bar{T}$ & $\bar{G}$ & $A$ & $\mathrm{C}$ \\
\hline $\begin{array}{l}\text { East- } \\
\text { Asia } \\
\end{array}$ & \begin{tabular}{|c|}
$\begin{array}{c}\text { Japane } \\
\text { se }\end{array}$ \\
\end{tabular} & \begin{tabular}{|c|} 
JPN_JK1 \\
850 \\
\end{tabular} & $\mathrm{~T}$ & $T$ & $\mathrm{G}$ & $C$ & $\bar{A}$ & $A$ & $\mathrm{C}$ & $C$ & $A$ & $\bar{T}$ & A & $\mathrm{T}$ & $\bar{T}$ & $\bar{T}$ & $\mathrm{G}$ & $\bar{A}$ & $\mathrm{C}$ \\
\hline $\begin{array}{l}\text { East- } \\
\text { Asia } \\
\end{array}$ & $\begin{array}{c}\text { Japane } \\
\text { se }\end{array}$ & $\begin{array}{c}\text { JPN_JK1 } \\
850 \\
\end{array}$ & $\mathrm{~T}$ & $T$ & $\mathrm{G}$ & $C$ & $G$ & $A$ & $\mathrm{C}$ & $\mathrm{C}$ & $A$ & $T$ & A & $T$ & $\mathrm{~T}$ & $T$ & $G$ & $\bar{A}$ & $\mathrm{C}$ \\
\hline $\begin{array}{l}\text { East- } \\
\text { Asia } \\
\end{array}$ & $\begin{array}{c}\text { Japane } \\
\text { se }\end{array}$ & \begin{tabular}{|c|} 
JPN_JK1 \\
851 \\
\end{tabular} & $\mathrm{~T}$ & $\bar{T}$ & $\bar{G}$ & $\bar{C}$ & $\bar{A}$ & $A$ & $\mathrm{C}$ & $\mathrm{C}$ & $A$ & $\bar{T}$ & $\bar{A}$ & $\bar{T}$ & $\bar{T}$ & $T$ & $\mathrm{G}$ & $\bar{A}$ & $\mathrm{C}$ \\
\hline $\begin{array}{l}\text { East- } \\
\text { Asia }\end{array}$ & $\begin{array}{c}\text { Japane } \\
\text { se }\end{array}$ & $\begin{array}{c}\text { JPN_JK1 } \\
8 \overline{51}\end{array}$ & $\mathrm{~T}$ & T & $\mathrm{G}$ & C & $\mathrm{G}$ & A & C & C & A & $\mathrm{T}$ & A & $\mathrm{T}$ & $\bar{T}$ & $\bar{T}$ & $G$ & A & $\mathrm{C}$ \\
\hline $\begin{array}{l}\text { East- } \\
\text { Asia }\end{array}$ & $\begin{array}{c}\text { Japane } \\
\text { se }\end{array}$ & $\begin{array}{c}\text { JPN_JK1 } \\
852 \\
\end{array}$ & $\mathrm{~T}$ & $T$ & $\mathrm{G}$ & $C$ & A & A & $\mathrm{C}$ & $\mathrm{C}$ & $A$ & $T$ & A & $\mathrm{T}$ & $T$ & $T$ & $\mathrm{G}$ & $\bar{A}$ & $\mathrm{C}$ \\
\hline $\begin{array}{l}\text { East- } \\
\text { Asia } \\
\end{array}$ & $\begin{array}{c}\text { Japane } \\
\text { se }\end{array}$ & \begin{tabular}{|c|} 
JPN_JK1 \\
852 \\
\end{tabular} & $\mathrm{~T}$ & $T$ & $\mathrm{G}$ & $C$ & $G$ & $A$ & $\mathrm{C}$ & $C$ & $A$ & $T$ & A & $T$ & $T$ & $T$ & $G$ & A & $\mathrm{C}$ \\
\hline $\begin{array}{l}\text { East- } \\
\text { Asia }\end{array}$ & $\begin{array}{c}\text { Japane } \\
\text { se }\end{array}$ & \begin{tabular}{|c|} 
JPN_JK1 \\
853
\end{tabular} & $\mathrm{~T}$ & $\mathrm{~T}$ & $\mathrm{G}$ & C & A & A & C & C & A & $\mathrm{T}$ & A & $\mathrm{T}$ & $\mathrm{T}$ & $\mathrm{T}$ & $\mathrm{G}$ & A & C \\
\hline $\begin{array}{l}\text { East- } \\
\text { Asia } \\
\end{array}$ & \begin{tabular}{|c|}
$\begin{array}{c}\text { Japane } \\
\text { se }\end{array}$ \\
\end{tabular} & \begin{tabular}{|c|} 
JPN_JK1 \\
853 \\
\end{tabular} & $\mathrm{~T}$ & $\mathrm{~T}$ & $\mathrm{G}$ & $C$ & $\bar{G}$ & $A$ & C & $C$ & $A$ & $\bar{T}$ & $A$ & $\mathrm{~T}$ & $T$ & $T$ & $\mathrm{G}$ & $\bar{A}$ & $\mathrm{C}$ \\
\hline $\begin{array}{l}\text { East- } \\
\text { Asia } \\
\end{array}$ & $\begin{array}{c}\text { Japane } \\
\text { se }\end{array}$ & \begin{tabular}{|c|} 
JPN_JK1 \\
856 \\
\end{tabular} & $\mathrm{~T}$ & $T$ & $\bar{G}$ & $C$ & $\bar{G}$ & $\bar{A}$ & $\mathrm{C}$ & $\mathrm{C}$ & $A$ & $\mathrm{~T}$ & $\bar{A}$ & $\mathrm{~T}$ & $T$ & $T$ & $\mathrm{G}$ & $\bar{A}$ & $\mathrm{C}$ \\
\hline $\begin{array}{l}\text { East- } \\
\text { Asia }\end{array}$ & $\begin{array}{c}\text { Japane } \\
\text { se }\end{array}$ & \begin{tabular}{|c|} 
JPN_JK1 \\
856
\end{tabular} & $\mathrm{~T}$ & $\mathrm{~T}$ & $G$ & $C$ & $G$ & A & $C$ & C & A & $\mathrm{T}$ & A & $\mathrm{T}$ & $\mathrm{T}$ & $\mathrm{T}$ & $\mathrm{G}$ & A & $C$ \\
\hline $\begin{array}{l}\text { East- } \\
\text { Asia } \\
\end{array}$ & \begin{tabular}{|c|}
$\begin{array}{c}\text { Japane } \\
\text { se }\end{array}$ \\
\end{tabular} & \begin{tabular}{|c|} 
JPN_JK1 \\
857 \\
\end{tabular} & $\mathrm{~T}$ & $\mathrm{~T}$ & $\mathrm{G}$ & C & $\bar{A}$ & $A$ & $\mathrm{C}$ & $\mathrm{C}$ & $A$ & $\bar{T}$ & $A$ & $\bar{T}$ & $\bar{T}$ & $\bar{T}$ & $\bar{G}$ & $A$ & $C$ \\
\hline $\begin{array}{l}\text { East- } \\
\text { Asia } \\
\end{array}$ & $\begin{array}{c}\text { Japane } \\
\text { se }\end{array}$ & $\begin{array}{c}\text { JPN_JK1 } \\
857 \\
\end{array}$ & $\mathrm{~T}$ & $T$ & $\bar{G}$ & $C$ & $\bar{G}$ & $\bar{A}$ & $\mathrm{C}$ & $\mathrm{C}$ & $A$ & $\mathrm{~T}$ & $\bar{A}$ & $\mathrm{~T}$ & $\mathrm{~T}$ & $\mathrm{~T}$ & $\mathrm{G}$ & $\bar{A}$ & $\mathrm{C}$ \\
\hline $\begin{array}{l}\text { East- } \\
\text { Asia }\end{array}$ & $\begin{array}{c}\text { Japane } \\
\text { se }\end{array}$ & \begin{tabular}{|c|} 
JPN_JK1 \\
860
\end{tabular} & $\mathrm{~T}$ & $T$ & $\mathrm{G}$ & C & $\bar{G}$ & $A$ & $C$ & $C$ & $A$ & $T$ & $\mathrm{G}$ & $\mathrm{T}$ & $\mathrm{T}$ & $\mathrm{T}$ & $\mathrm{G}$ & A & $C$ \\
\hline $\begin{array}{l}\text { East- } \\
\text { Asia } \\
\end{array}$ & \begin{tabular}{|c|}
$\begin{array}{c}\text { Japane } \\
\text { se }\end{array}$ \\
\end{tabular} & \begin{tabular}{|c|} 
JPN_JK1 \\
860 \\
\end{tabular} & $\mathrm{~T}$ & $\mathrm{~T}$ & $\bar{G}$ & $C$ & $\bar{G}$ & $A$ & $\mathrm{C}$ & $C$ & $A$ & $\mathrm{~T}$ & $A$ & $\bar{T}$ & $\mathrm{~T}$ & $\mathrm{~T}$ & $\bar{G}$ & $A$ & $C$ \\
\hline $\begin{array}{l}\text { East- } \\
\text { Asia }\end{array}$ & $\begin{array}{c}\text { Japane } \\
\text { se }\end{array}$ & \begin{tabular}{|c} 
JPN_JK1 \\
863 \\
\end{tabular} & $\mathrm{~T}$ & $T$ & $G$ & $C$ & $G$ & $A$ & $\mathrm{C}$ & $\mathrm{C}$ & $A$ & $T$ & A & $\mathrm{T}$ & $\mathrm{T}$ & $T$ & $\mathrm{G}$ & $\bar{A}$ & $\mathrm{C}$ \\
\hline $\begin{array}{l}\text { East- } \\
\text { Asia } \\
\end{array}$ & $\begin{array}{c}\text { Japane } \\
\text { se }\end{array}$ & \begin{tabular}{|c|} 
JPN_JK1 1 \\
863 \\
\end{tabular} & $\mathrm{~T}$ & $T$ & $\mathrm{G}$ & $C$ & $G$ & $A$ & $\mathrm{C}$ & $C$ & $A$ & $T$ & $\bar{A}$ & $\mathrm{~T}$ & $\mathrm{~T}$ & $\mathrm{~T}$ & $G$ & A & $C$ \\
\hline $\begin{array}{l}\text { East- } \\
\text { Asia } \\
\end{array}$ & \begin{tabular}{|c} 
Japane \\
se
\end{tabular} & \begin{tabular}{|c|} 
JPN_TM \\
KH
\end{tabular} & $\mathrm{T}$ & $\mathrm{T}$ & $\mathrm{G}$ & $C$ & $A$ & $A$ & $\mathrm{C}$ & $C$ & $A$ & $T$ & $A$ & $\bar{T}$ & $\bar{T}$ & $\bar{T}$ & $G$ & A & $C$ \\
\hline $\begin{array}{l}\text { East- } \\
\text { Asia } \\
\end{array}$ & $\begin{array}{c}\begin{array}{c}\text { Japane } \\
\text { se }\end{array} \\
\end{array}$ & \begin{tabular}{|c|} 
JPN_TM \\
$\mathbf{K} \overline{\mathrm{H}}$
\end{tabular} & $\bar{T}$ & $\bar{T}$ & $\bar{G}$ & $C$ & $\bar{G}$ & $\bar{A}$ & $\mathrm{C}$ & $C$ & $A$ & $\mathrm{~T}$ & A & $\mathrm{T}$ & $\mathrm{T}$ & $T$ & $\bar{G}$ & A & $\mathrm{C}$ \\
\hline $\begin{array}{c}\text { Europ } \\
\mathrm{e}\end{array}$ & \begin{tabular}{|c}
$\begin{array}{c}\text { Chuvas } \\
\mathrm{h}\end{array}$ \\
\end{tabular} & \begin{tabular}{|c|c|} 
CHU_JK \\
4051
\end{tabular} & $\mathrm{~T}$ & $T$ & $\mathrm{G}$ & $C$ & $A$ & $A$ & C & $C$ & $A$ & $T$ & $\bar{A}$ & $\mathrm{~T}$ & $\mathrm{G}$ & $T$ & $G$ & A & C \\
\hline $\begin{array}{c}\text { Europ } \\
\mathrm{e}\end{array}$ & \begin{tabular}{|c|c|}
$\begin{array}{c}\text { Chuvas } \\
\mathrm{h}\end{array}$ \\
\end{tabular} & \begin{tabular}{|c|} 
CHU_JK \\
4051
\end{tabular} & $\mathrm{~T}$ & $T$ & C & $\bar{C}$ & $\bar{G}$ & $\bar{A}$ & $\mathrm{C}$ & $\mathrm{C}$ & $A$ & $\bar{T}$ & $\bar{A}$ & $\bar{T}$ & $\bar{T}$ & $\bar{T}$ & $\bar{G}$ & $A$ & $\mathrm{C}$ \\
\hline Europ & Chuvas & CHU_JK & $\mathrm{T}$ & $\mathrm{T}$ & $G$ & C & A & A & $C$ & $C$ & A & $\mathrm{T}$ & A & $\mathrm{T}$ & $\mathrm{G}$ & $\mathrm{T}$ & $\mathrm{G}$ & A & $C$ \\
\hline
\end{tabular}

John Wiley $1 Q$ Sons, Inc. 


\begin{tabular}{|c|c|c|c|c|c|c|c|c|c|c|c|c|c|c|c|c|c|c|c|}
\hline $\mathrm{e}$ & $\mathrm{h}$ & 4052 & & & & & & & & & & & & & & & & & \\
\hline $\begin{array}{c}\text { Europ } \\
\mathrm{e}\end{array}$ & $\begin{array}{c}\text { Chuvas } \\
\mathrm{h}\end{array}$ & $\begin{array}{c}\text { CHU_JK } \\
4052\end{array}$ & $\mathrm{~T}$ & $T$ & $G$ & $\mathrm{C}$ & $\mathrm{G}$ & $\bar{A}$ & C & $\mathrm{C}$ & $A$ & $T$ & A & $\mathrm{T}$ & $T$ & $\mathrm{~T}$ & $\mathrm{G}$ & $\bar{A}$ & C \\
\hline $\begin{array}{c}\text { Europ } \\
\mathrm{e}\end{array}$ & $\begin{array}{c}\text { Chuvas } \\
\mathrm{h}\end{array}$ & $\begin{array}{c}\text { CHU_JK } \\
4053\end{array}$ & $\mathrm{~T}$ & $\mathrm{~T}$ & $G$ & $\mathrm{C}$ & $A$ & $\bar{A}$ & C & $\mathrm{C}$ & $A$ & $\mathrm{~T}$ & $\bar{A}$ & $T$ & $\mathrm{G}$ & $\bar{T}$ & $\mathrm{G}$ & $\bar{A}$ & $C$ \\
\hline $\begin{array}{c}\text { Europ } \\
\mathrm{e}\end{array}$ & $\begin{array}{c}\text { Chuvas } \\
\mathrm{h}\end{array}$ & $\begin{array}{c}\text { CHU_JK } \\
4053\end{array}$ & $\mathrm{~T}$ & $T$ & $\bar{G}$ & $\mathrm{C}$ & $A$ & A & $C$ & $\mathrm{C}$ & $A$ & $T$ & A & $\mathrm{T}$ & $\bar{G}$ & $\mathrm{~T}$ & $\mathrm{G}$ & $A$ & $C$ \\
\hline $\begin{array}{c}\text { Europ } \\
\mathrm{e}\end{array}$ & $\begin{array}{c}\text { Chuvas } \\
\mathrm{h}\end{array}$ & $\underset{4054}{\mathrm{CHU} J \mathrm{JK}}$ & $\mathrm{T}$ & $\mathrm{T}$ & $G$ & $C$ & $\mathrm{~A}$ & $\mathrm{~A}$ & $C$ & $\mathrm{C}$ & $A$ & $\mathrm{~T}$ & $\mathrm{~A}$ & $\mathrm{~T}$ & $\mathrm{G}$ & $\mathrm{T}$ & $\mathrm{G}$ & $\mathrm{A}$ & $C$ \\
\hline $\begin{array}{c}\text { Europ } \\
\mathrm{e}\end{array}$ & $\begin{array}{c}\text { Chuvas } \\
\mathrm{h}\end{array}$ & $\begin{array}{c}\text { CHU_JK } \\
4054\end{array}$ & $\mathrm{~T}$ & $\mathrm{~T}$ & $C$ & $\mathrm{C}$ & $\bar{G}$ & $\mathrm{~A}$ & $C$ & $\mathrm{C}$ & $A$ & $\mathrm{~T}$ & $A$ & $\mathrm{~T}$ & $\mathrm{~T}$ & $\mathrm{~T}$ & $\bar{G}$ & $A$ & $C$ \\
\hline $\begin{array}{c}\text { Europ } \\
\mathrm{e}\end{array}$ & $\begin{array}{c}\text { Chuvas } \\
\mathrm{h}\end{array}$ & $\underset{4055}{\mathrm{CHU} J \mathrm{JK}}$ & $\mathrm{T}$ & $\mathrm{T}$ & $\mathrm{G}$ & $C$ & $A$ & $A$ & $C$ & $\mathrm{C}$ & $A$ & $\mathrm{~T}$ & A & $\mathrm{T}$ & $\mathrm{G}$ & $\mathrm{T}$ & $\mathrm{G}$ & A & $C$ \\
\hline $\begin{array}{c}\text { Europ } \\
\mathrm{e}\end{array}$ & $\begin{array}{c}\text { Chuvas } \\
\mathrm{h}\end{array}$ & $\underset{4055}{\text { CHU_JK }}$ & $\mathrm{T}$ & $T$ & $G$ & C & $A$ & $A$ & $C$ & $\mathrm{C}$ & $A$ & $T$ & A & $T$ & $\mathrm{G}$ & $\mathrm{T}$ & $\mathrm{G}$ & $A$ & C \\
\hline $\begin{array}{c}\text { Europ } \\
\mathrm{e}\end{array}$ & $\begin{array}{c}\text { Chuvas } \\
\mathrm{h}\end{array}$ & $\begin{array}{c}\text { CHU_JK } \\
4056\end{array}$ & $\mathrm{~T}$ & $\mathrm{~T}$ & $G$ & $C$ & $\bar{A}$ & $A$ & $C$ & $C$ & $A$ & $\mathrm{~T}$ & $A$ & $\mathrm{~T}$ & $\mathrm{~T}$ & $\mathrm{~T}$ & $\mathrm{G}$ & $A$ & $C$ \\
\hline $\begin{array}{c}\text { Europ } \\
\mathrm{e}\end{array}$ & $\begin{array}{c}\text { Chuvas } \\
\mathrm{h}\end{array}$ & $\underset{4056}{\mathrm{CHU} J \mathrm{JK}}$ & C & $\mathrm{T}$ & $G$ & C & $\mathrm{G}$ & $A$ & $C$ & $\mathrm{C}$ & $A$ & $\mathrm{~T}$ & $A$ & $\mathrm{~T}$ & $\mathrm{~T}$ & $\mathrm{~T}$ & $\mathrm{G}$ & $A$ & C \\
\hline $\begin{array}{c}\text { Europ } \\
\mathrm{e}\end{array}$ & $\begin{array}{c}\text { Chuvas } \\
\mathrm{h}\end{array}$ & $\underset{4057}{\text { CHU_JK }}$ & $\mathrm{T}$ & $T$ & $G$ & C & $A$ & A & $C$ & $\mathrm{C}$ & $A$ & $\mathrm{~T}$ & A & $\mathrm{T}$ & $\mathrm{T}$ & $\mathrm{T}$ & $\mathrm{G}$ & $A$ & C \\
\hline $\begin{array}{c}\text { Europ } \\
\mathrm{e}\end{array}$ & $\begin{array}{c}\text { Chuvas } \\
\mathrm{h}\end{array}$ & $\underset{4057}{\text { CHU_JK }}$ & $\mathrm{T}$ & $T$ & $\mathrm{G}$ & $C$ & $\bar{G}$ & $\mathrm{~A}$ & C & $C$ & $\mathrm{~A}$ & $\mathrm{~T}$ & $\mathrm{~A}$ & $\mathrm{~T}$ & $\mathrm{~T}$ & $\mathrm{~T}$ & $\bar{G}$ & $\mathrm{~A}$ & $C$ \\
\hline $\begin{array}{c}\text { Europ } \\
\mathrm{e}\end{array}$ & $\begin{array}{c}\text { Chuvas } \\
\mathrm{h}\end{array}$ & $\begin{array}{c}\text { CHU_JK } \\
4058\end{array}$ & $\mathrm{~T}$ & $\mathrm{~T}$ & $G$ & $\mathrm{C}$ & $A$ & $A$ & C & $\mathrm{C}$ & A & $T$ & $\bar{A}$ & $T$ & $G$ & $\mathrm{~T}$ & $\mathrm{G}$ & $A$ & $C$ \\
\hline $\begin{array}{c}\text { Europ } \\
\mathrm{e}\end{array}$ & $\begin{array}{c}\text { Chuvas } \\
\mathrm{h}\end{array}$ & $\begin{array}{c}\text { CHU_JK } \\
4058\end{array}$ & $\mathrm{~T}$ & $T$ & $\mathrm{G}$ & $C$ & $A$ & $\bar{A}$ & $C$ & $\mathrm{C}$ & $A$ & $T$ & A & $T$ & $\mathrm{~T}$ & $\mathrm{~T}$ & $\mathrm{G}$ & $\bar{A}$ & $C$ \\
\hline $\begin{array}{c}\text { Europ } \\
\mathrm{e}\end{array}$ & $\begin{array}{c}\text { Chuvas } \\
\mathrm{h}\end{array}$ & $\underset{4059}{\text { CHU_JK }}$ & $\mathrm{T}$ & $\mathrm{T}$ & $G$ & $\bar{C}$ & $\bar{A}$ & $\mathrm{~A}$ & C & $\mathrm{C}$ & $\mathrm{A}$ & $T$ & $\mathrm{~A}$ & $\mathrm{~T}$ & $\bar{G}$ & $\mathrm{~T}$ & $\bar{G}$ & $\bar{A}$ & $C$ \\
\hline $\begin{array}{c}\text { Europ } \\
\mathrm{e}\end{array}$ & $\begin{array}{c}\text { Chuvas } \\
\mathrm{h}\end{array}$ & $\begin{array}{c}\text { CHU_JK } \\
4059\end{array}$ & $\mathrm{C}$ & $T$ & $\bar{G}$ & C & $\mathrm{G}$ & $\bar{A}$ & C & $\mathrm{C}$ & $A$ & $T$ & A & $T$ & $\mathrm{~T}$ & $\mathrm{~T}$ & $\mathrm{G}$ & $A$ & $C$ \\
\hline $\begin{array}{c}\text { Europ } \\
\mathrm{e}\end{array}$ & $\begin{array}{c}\text { Chuvas } \\
\mathrm{h}\end{array}$ & $\begin{array}{c}\mathrm{CHU \_ JK} \\
4060\end{array}$ & $\mathrm{~T}$ & $T$ & $G$ & $C$ & $\mathrm{G}$ & $\bar{A}$ & $C$ & $\mathrm{C}$ & $A$ & $T$ & $A$ & $T$ & $\mathrm{~T}$ & $\mathrm{~T}$ & $\mathrm{G}$ & A & $C$ \\
\hline $\begin{array}{c}\text { Europ } \\
\mathrm{e}\end{array}$ & $\begin{array}{c}\text { Chuvas } \\
\mathrm{h}\end{array}$ & $\underset{4060}{\mathrm{CHU} J \mathrm{JK}}$ & $\mathrm{T}$ & $\mathrm{T}$ & $G$ & C & $\mathrm{G}$ & $\mathrm{A}$ & $C$ & $\mathrm{C}$ & A & $\mathrm{T}$ & $A$ & $\mathrm{~T}$ & $\mathrm{~T}$ & $\mathrm{~T}$ & $\mathrm{G}$ & $A$ & $C$ \\
\hline $\begin{array}{c}\text { Europ } \\
\mathrm{e}\end{array}$ & $\begin{array}{c}\text { Chuvas } \\
\mathrm{h}\end{array}$ & $\begin{array}{c}\text { CHU_JK } \\
4061\end{array}$ & $\mathrm{~T}$ & $T$ & $\mathrm{G}$ & $C$ & $\mathrm{G}$ & $A$ & C & $\mathrm{C}$ & $A$ & $T$ & $\bar{A}$ & $\mathrm{~T}$ & $T$ & $T$ & $\mathrm{G}$ & $A$ & C \\
\hline $\begin{array}{c}\text { Europ } \\
\mathrm{e}\end{array}$ & $\begin{array}{c}\text { Chuvas } \\
\mathrm{h}\end{array}$ & $\begin{array}{c}\text { CHU_JK } \\
4061\end{array}$ & $\mathrm{~T}$ & $T$ & $G$ & $C$ & $\mathrm{G}$ & $A$ & C & $\mathrm{C}$ & A & $\mathrm{T}$ & A & $\mathrm{T}$ & $\mathrm{T}$ & $\mathrm{T}$ & $\mathrm{G}$ & $A$ & C \\
\hline $\begin{array}{c}\text { Europ } \\
\mathrm{e}\end{array}$ & $\begin{array}{c}\text { Chuvas } \\
\mathrm{h}\end{array}$ & $\underset{4062}{\mathrm{CHU} J \mathrm{JK}}$ & $\mathrm{T}$ & $T$ & $G$ & $\mathrm{C}$ & $A$ & $A$ & $C$ & $\mathrm{C}$ & $\bar{A}$ & $T$ & $\bar{A}$ & $T$ & $G$ & $T$ & $\mathrm{G}$ & $\bar{A}$ & C \\
\hline $\begin{array}{c}\text { Europ } \\
\mathrm{e}\end{array}$ & $\begin{array}{c}\text { Chuvas } \\
\mathrm{h}\end{array}$ & $\begin{array}{c}\text { CHU_JK } \\
4062\end{array}$ & $\mathrm{~T}$ & $T$ & $G$ & C & $A$ & $A$ & C & $\mathrm{C}$ & $A$ & $T$ & $\bar{A}$ & $\mathrm{~T}$ & $G$ & $\mathrm{~T}$ & $\mathrm{G}$ & A & C \\
\hline $\begin{array}{c}\text { Europ } \\
\mathrm{e}\end{array}$ & $\begin{array}{c}\text { Chuvas } \\
\mathrm{h}\end{array}$ & $\underset{4063}{\mathrm{CHU} J \mathrm{JK}}$ & $\mathrm{T}$ & $T$ & $G$ & $C$ & $\mathrm{G}$ & $A$ & C & $\mathrm{C}$ & A & $\mathrm{T}$ & $\bar{A}$ & $T$ & $\mathrm{~T}$ & $T$ & $\mathrm{G}$ & A & C \\
\hline Europ & Chuvas & CHU_JK & $T$ & $T$ & $G$ & $\mathrm{C}$ & $\mathrm{G}$ & $T$ & C & $\mathrm{C}$ & $\bar{A}$ & $T$ & A & $T$ & $T$ & $T$ & $\mathrm{G}$ & $\mathrm{A}$ & C \\
\hline
\end{tabular}

John Wiley \& Sons, Inc. 


\begin{tabular}{|c|c|c|c|c|c|c|c|c|c|c|c|c|c|c|c|c|c|c|c|}
\hline $\mathrm{e}$ & $\mathrm{h}$ & 4063 & & & & & & & & & & & & & & & & & \\
\hline $\begin{array}{c}\text { Europ } \\
\mathrm{e}\end{array}$ & $\begin{array}{c}\begin{array}{c}\text { Chuvas } \\
\mathrm{h}\end{array} \\
\end{array}$ & $\begin{array}{c}\text { CHU_JK } \\
4064\end{array}$ & $\mathrm{~T}$ & $\mathrm{~T}$ & $\bar{G}$ & $\mathrm{C}$ & $\bar{A}$ & $\bar{A}$ & $C$ & $\mathrm{C}$ & $A$ & $T$ & $A$ & $\mathrm{~T}$ & $\mathrm{~T}$ & $\mathrm{~T}$ & $\mathrm{G}$ & $\mathrm{A}$ & $\mathrm{C}$ \\
\hline $\begin{array}{c}\text { Europ } \\
\mathrm{e}\end{array}$ & $\begin{array}{c}\text { Chuvas } \\
\mathrm{h}\end{array}$ & $\underset{4064}{\mathrm{CHU} J \mathrm{JK}}$ & $\mathrm{T}$ & $\mathrm{T}$ & $\mathrm{G}$ & $\mathrm{C}$ & $G$ & A & $\mathrm{C}$ & C & A & $T$ & $A$ & $\mathrm{~T}$ & $T$ & $T$ & $\mathrm{G}$ & $A$ & C \\
\hline $\begin{array}{c}\text { Europ } \\
\mathrm{e}\end{array}$ & $\begin{array}{c}\text { Chuvas } \\
\text { h }\end{array}$ & $\underset{4065}{\text { CHU_JK }}$ & $\mathrm{T}$ & $\mathrm{T}$ & $\bar{G}$ & $\mathrm{C}$ & $\mathrm{A}$ & $\bar{A}$ & $C$ & $C$ & $\bar{A}$ & $\bar{T}$ & $A$ & $\mathrm{~T}$ & $\mathrm{G}$ & $\bar{T}$ & $\bar{G}$ & $\mathrm{~A}$ & $\mathrm{C}$ \\
\hline $\begin{array}{c}\text { Europ } \\
\mathrm{e}\end{array}$ & $\begin{array}{c}\text { Chuvas } \\
\text { h }\end{array}$ & $\underset{4065}{\text { CHU_JK }}$ & $\mathrm{T}$ & $\mathrm{T}$ & $C$ & $C$ & $\mathrm{G}$ & $\mathrm{A}$ & $C$ & $C$ & $\mathrm{~A}$ & $T$ & $A$ & $\mathrm{~T}$ & $\mathrm{~T}$ & $\mathrm{~T}$ & $\mathrm{G}$ & $A$ & $\mathrm{C}$ \\
\hline $\begin{array}{c}\text { Europ } \\
\mathrm{e}\end{array}$ & $\begin{array}{c}\text { Chuvas } \\
\mathrm{h}\end{array}$ & $\begin{array}{c}\text { CHU_JK } \\
4066\end{array}$ & $\mathrm{~T}$ & $\mathrm{~T}$ & $\mathrm{G}$ & $\mathrm{C}$ & $\bar{A}$ & $\bar{A}$ & $\mathrm{C}$ & C & $\bar{A}$ & $\mathrm{~T}$ & $A$ & $\mathrm{~T}$ & $\mathrm{G}$ & $\mathrm{T}$ & $G$ & $A$ & $\mathrm{C}$ \\
\hline $\begin{array}{c}\text { Europ } \\
\mathrm{e}\end{array}$ & $\begin{array}{c}\text { Chuvas } \\
\mathrm{h}\end{array}$ & $\underset{4066}{\mathrm{CHU} J \mathrm{JK}}$ & $\mathrm{T}$ & $\mathrm{T}$ & C & $\mathrm{C}$ & $G$ & A & C & C & A & $T$ & $A$ & $\mathrm{~T}$ & $\mathrm{~T}$ & $T$ & $G$ & $A$ & C \\
\hline $\begin{array}{c}\text { Europ } \\
\mathrm{e}\end{array}$ & $\begin{array}{c}\text { Chuvas } \\
\mathrm{h}\end{array}$ & $\begin{array}{c}\text { CHU_JK } \\
4067\end{array}$ & $\mathrm{~T}$ & $\mathrm{~T}$ & $\mathrm{G}$ & $C$ & $\mathrm{G}$ & $A$ & $C$ & $C$ & $A$ & $\mathrm{~T}$ & $A$ & $\mathrm{~T}$ & $\mathrm{~T}$ & $T$ & $\mathrm{G}$ & $\mathrm{A}$ & $\mathrm{C}$ \\
\hline $\begin{array}{c}\text { Europ } \\
\mathrm{e}\end{array}$ & \begin{tabular}{|c}
$\begin{array}{c}\text { Chuvas } \\
\mathrm{h}\end{array}$ \\
\end{tabular} & $\begin{array}{c}\text { CHU_JK } \\
4067\end{array}$ & $\mathrm{C}$ & $\mathrm{T}$ & $\mathrm{G}$ & $\mathrm{C}$ & $\mathrm{G}$ & $\mathrm{A}$ & $C$ & $C$ & $A$ & $\mathrm{~T}$ & $\mathrm{~A}$ & $\mathrm{~T}$ & $\mathrm{~T}$ & $\mathrm{~T}$ & $\mathrm{G}$ & $\mathrm{A}$ & $\mathrm{C}$ \\
\hline $\begin{array}{c}\text { Europ } \\
\mathrm{e}\end{array}$ & $\begin{array}{c}\text { Chuvas } \\
\mathrm{h}\end{array}$ & $\underset{4068}{\mathrm{CHU} J \mathrm{JK}}$ & $\mathrm{T}$ & $\mathrm{T}$ & $\mathrm{G}$ & $\mathrm{C}$ & $A$ & A & C & C & A & $T$ & $A$ & $\mathrm{~T}$ & $\mathrm{G}$ & $T$ & $\mathrm{G}$ & $A$ & C \\
\hline $\begin{array}{c}\text { Europ } \\
\mathrm{e}\end{array}$ & $\begin{array}{c}\text { Chuvas } \\
\mathrm{h}\end{array}$ & $\begin{array}{c}\text { CHU_JK } \\
4068\end{array}$ & $\mathrm{~T}$ & $\mathrm{~T}$ & $\mathrm{G}$ & $C$ & $\mathrm{~A}$ & $\mathrm{~A}$ & $C$ & $C$ & $\mathrm{~A}$ & $T$ & $A$ & $T$ & $\mathrm{~T}$ & $\mathrm{~T}$ & $\mathrm{G}$ & $\mathrm{A}$ & $\mathrm{C}$ \\
\hline $\begin{array}{c}\text { Europ } \\
\mathrm{e}\end{array}$ & \begin{tabular}{|c|c|}
$\begin{array}{c}\text { Chuvas } \\
\mathrm{h}\end{array}$ \\
\end{tabular} & $\begin{array}{c}\text { CHU_JK } \\
4069\end{array}$ & $\mathrm{~T}$ & $\mathrm{~T}$ & $\bar{G}$ & $\mathrm{C}$ & $\mathrm{A}$ & $\mathrm{A}$ & C & $C$ & $A$ & $\mathrm{~T}$ & $A$ & $\mathrm{~T}$ & $T$ & $\mathrm{~T}$ & $\mathrm{G}$ & $\mathrm{A}$ & C \\
\hline $\begin{array}{c}\text { Europ } \\
\mathrm{e}\end{array}$ & $\begin{array}{c}\text { Chuvas } \\
\mathrm{h}\end{array}$ & $\underset{4069}{\mathrm{CHU} J \mathrm{JK}}$ & $\mathrm{T}$ & $\mathrm{T}$ & $G$ & $\mathrm{C}$ & $\mathrm{G}$ & A & $\mathrm{C}$ & C & A & $\mathrm{T}$ & $A$ & $\mathrm{~T}$ & $\mathrm{~T}$ & $T$ & $\mathrm{G}$ & $A$ & C \\
\hline $\begin{array}{c}\text { Europ } \\
\mathrm{e}\end{array}$ & $\begin{array}{c}\text { Chuvas } \\
\mathrm{h}\end{array}$ & $\underset{4070}{\text { CHU_JK }}$ & $\mathrm{T}$ & $\mathrm{T}$ & $\mathrm{G}$ & $\mathrm{C}$ & $\bar{A}$ & $\bar{A}$ & $C$ & C & $\bar{A}$ & $\bar{T}$ & $A$ & $\mathrm{~T}$ & $\mathrm{G}$ & $\mathrm{T}$ & $\mathrm{G}$ & $\mathrm{A}$ & $\mathrm{C}$ \\
\hline $\begin{array}{c}\text { Europ } \\
\mathrm{e}\end{array}$ & $\begin{array}{c}\text { Chuvas } \\
\mathrm{h}\end{array}$ & $\begin{array}{c}\text { CHU_JK } \\
4070\end{array}$ & $\mathrm{~T}$ & $\mathrm{~T}$ & $\bar{G}$ & $\mathrm{C}$ & $\mathrm{A}$ & $\bar{A}$ & $\mathrm{C}$ & C & $A$ & $T$ & $A$ & $T$ & $\mathrm{~T}$ & $\mathrm{~T}$ & $\mathrm{G}$ & $A$ & $\mathrm{C}$ \\
\hline $\begin{array}{c}\text { Europ } \\
\mathrm{e}\end{array}$ & $\begin{array}{c}\text { Chuvas } \\
\mathrm{h}\end{array}$ & $\underset{4071}{\text { CHU_JK }}$ & $\mathrm{T}$ & $\mathrm{T}$ & $\mathrm{G}$ & $\mathrm{C}$ & $G$ & A & C & $C$ & A & $T$ & $A$ & $\mathrm{~T}$ & $T$ & $\mathrm{~T}$ & $\mathrm{G}$ & $A$ & C \\
\hline $\begin{array}{c}\text { Europ } \\
\mathrm{e}\end{array}$ & $\begin{array}{c}\text { Chuvas } \\
\text { h }\end{array}$ & $\underset{4071}{\text { CHU_JK }}$ & $\mathrm{T}$ & $\mathrm{T}$ & $\mathrm{G}$ & $\mathrm{C}$ & $\bar{G}$ & $\bar{A}$ & $\mathrm{C}$ & C & $\bar{A}$ & $\bar{T}$ & $A$ & $T$ & $\bar{T}$ & $T$ & $\mathrm{G}$ & $A$ & $\mathrm{C}$ \\
\hline $\begin{array}{c}\text { Europ } \\
\mathrm{e}\end{array}$ & $\begin{array}{c}\text { Chuvas } \\
\mathrm{h}\end{array}$ & $\begin{array}{c}\text { CHU_JK } \\
4072\end{array}$ & $T$ & $\mathrm{~T}$ & $\mathrm{G}$ & $\mathrm{C}$ & $G$ & A & $\mathrm{C}$ & C & A & $T$ & $A$ & $T$ & $\mathrm{~T}$ & $T$ & $\mathrm{G}$ & $A$ & $\mathrm{C}$ \\
\hline $\begin{array}{c}\text { Europ } \\
\mathrm{e}\end{array}$ & $\begin{array}{c}\begin{array}{c}\text { Chuvas } \\
\mathrm{h}\end{array} \\
\end{array}$ & $\begin{array}{c}\text { CHU_JK } \\
4072\end{array}$ & $T$ & $\mathrm{~T}$ & $\mathrm{G}$ & $\mathrm{C}$ & $G$ & A & C & C & A & $T$ & $A$ & $\mathrm{~T}$ & $T$ & $\mathrm{~T}$ & $\mathrm{G}$ & $A$ & C \\
\hline $\begin{array}{c}\text { Europ } \\
\mathrm{e}\end{array}$ & $\begin{array}{c}\text { Chuvas } \\
\mathrm{h}\end{array}$ & $\underset{4073}{\text { CHU_JK }}$ & $\mathrm{T}$ & $\mathrm{T}$ & $\mathrm{G}$ & $\mathrm{C}$ & A & A & C & C & A & $T$ & $A$ & $\mathrm{~T}$ & $T$ & $T$ & $\mathrm{G}$ & $A$ & $\mathrm{C}$ \\
\hline $\begin{array}{c}\text { Europ } \\
\mathrm{e}\end{array}$ & $\begin{array}{c}\text { Chuvas } \\
\mathrm{h}\end{array}$ & $\begin{array}{c}\text { CHU_JK } \\
4073\end{array}$ & $T$ & $\mathrm{~T}$ & $\mathrm{G}$ & $\mathrm{C}$ & $G$ & A & $\mathrm{C}$ & C & A & $T$ & $A$ & $\mathrm{~T}$ & $\mathrm{~T}$ & $T$ & $G$ & $A$ & $\mathrm{C}$ \\
\hline $\begin{array}{c}\text { Europ } \\
\mathrm{e}\end{array}$ & \begin{tabular}{|c}
$\begin{array}{c}\text { Chuvas } \\
\mathrm{h}\end{array}$ \\
\end{tabular} & $\begin{array}{c}\text { CHU_JK } \\
4074\end{array}$ & $T$ & $\mathrm{~T}$ & $\mathrm{G}$ & $\mathrm{C}$ & $G$ & A & C & C & A & $\mathrm{T}$ & $A$ & $\mathrm{~T}$ & $\mathrm{~T}$ & $\mathrm{~T}$ & $G$ & $A$ & C \\
\hline $\begin{array}{c}\text { Europ } \\
\mathrm{e}\end{array}$ & $\begin{array}{c}\text { Chuvas } \\
\mathrm{h}\end{array}$ & $\underset{4074}{\text { CHU_JK }}$ & $T$ & $\mathrm{~T}$ & $G$ & C & $G$ & $T$ & C & C & $\bar{A}$ & $T$ & $A$ & $\mathrm{~T}$ & $\mathrm{~T}$ & $T$ & $G$ & $A$ & $\mathrm{C}$ \\
\hline Europ & Danes & DAN_BK & $T$ & $\mathrm{~T}$ & $G$ & $\mathrm{C}$ & A & A & $\mathrm{C}$ & C & A & $T$ & $A$ & $\mathrm{~T}$ & $\mathrm{G}$ & $T$ & $G$ & $A$ & C \\
\hline
\end{tabular}

John Wiley \& Sons, Inc. 


\begin{tabular}{|c|c|c|c|c|c|c|c|c|c|c|c|c|c|c|c|c|c|c|c|}
\hline $\bar{e}$ & & 113 & & & & & & & & & & & & & & & & & \\
\hline $\begin{array}{c}\text { Europ } \\
\mathrm{e}\end{array}$ & Danes & $\begin{array}{c}\text { DAN_BK } \\
113\end{array}$ & $\begin{array}{cc}\mathrm{T} \\
\end{array}$ & $T$ & $\mathrm{G}$ & $\mathrm{C}$ & $\mathrm{G}$ & $\bar{A}$ & $C$ & $\mathrm{C}$ & $\mathrm{A}$ & $T$ & $\mathrm{~A}$ & $\mathrm{~T}$ & $\mathrm{~T}$ & $\mathrm{~T}$ & $\bar{G}$ & $A$ & $\mathrm{C}$ \\
\hline $\begin{array}{c}\text { Europ } \\
\mathrm{e}\end{array}$ & Danes & $\begin{array}{c}\text { DAN_BK } \\
116\end{array}$ & $\begin{array}{cl}\mathrm{T} \\
\end{array}$ & $T$ & $\mathrm{G}$ & $\mathrm{C}$ & $\mathrm{A}$ & $\mathrm{A}$ & $C$ & $\mathrm{C}$ & $\mathrm{A}$ & $T$ & $A$ & $\mathrm{~T}$ & $\mathrm{G}$ & $\mathrm{T}$ & $\mathrm{G}$ & $\mathrm{A}$ & $\mathrm{C}$ \\
\hline $\begin{array}{c}\text { Europ } \\
\mathrm{e}\end{array}$ & Danes & $\begin{array}{c}\text { DAN_BK } \\
116\end{array}$ & $\bar{T}$ & $\bar{T}$ & $\mathrm{G}$ & $\mathrm{C}$ & $\bar{A}$ & $\bar{A}$ & C & $\mathrm{C}$ & $A$ & $\bar{T}$ & $A$ & $\bar{T}$ & $\mathrm{G}$ & $\bar{T}$ & $\mathrm{G}$ & $\bar{A}$ & $\mathrm{C}$ \\
\hline $\begin{array}{c}\text { Europ } \\
\mathrm{e}\end{array}$ & Danes & $\begin{array}{c}\text { DAN_BK } \\
117\end{array}$ & $\mathrm{~T}$ & $T$ & $\mathrm{G}$ & $C$ & $A$ & $A$ & $C$ & $C$ & $\mathrm{~A}$ & $T$ & $\mathrm{~A}$ & $T$ & $G$ & $T$ & $\mathrm{G}$ & $\bar{A}$ & $\mathrm{C}$ \\
\hline $\begin{array}{c}\text { Europ } \\
\mathrm{e}\end{array}$ & Danes & $\begin{array}{c}\text { DAN_BK } \\
117\end{array}$ & $\begin{array}{lll}T \\
\end{array}$ & $T$ & $\mathrm{G}$ & $\mathrm{C}$ & $\mathrm{A}$ & $\bar{A}$ & C & $\mathrm{C}$ & $A$ & $T$ & $A$ & $\mathrm{~T}$ & $\mathrm{G}$ & $\mathrm{T}$ & $\mathrm{G}$ & $\bar{A}$ & $\mathrm{C}$ \\
\hline $\begin{array}{c}\text { Europ } \\
\mathrm{e}\end{array}$ & Danes & $\begin{array}{c}\text { DAN_BK } \\
119\end{array}$ & $\mathrm{~T}$ & $T$ & $\mathrm{G}$ & $C$ & $A$ & $A$ & $C$ & $C$ & $A$ & $\mathrm{~T}$ & $A$ & $T$ & $\mathrm{G}$ & $\mathrm{T}$ & $\mathrm{G}$ & $\bar{A}$ & $\mathrm{C}$ \\
\hline $\begin{array}{c}\text { Europ } \\
\mathrm{e}\end{array}$ & Danes & $\begin{array}{c}\text { DAN_BK } \\
119\end{array}$ & $\mathrm{~T}$ & $T$ & $\mathrm{G}$ & $C$ & A & $\mathrm{A}$ & $C$ & $C$ & $\mathrm{~A}$ & $T$ & $\mathrm{~A}$ & $T$ & $T$ & $T$ & $\mathrm{G}$ & $C$ & $\mathrm{C}$ \\
\hline $\begin{array}{c}\text { Europ } \\
\mathrm{e}\end{array}$ & Danes & $\begin{array}{c}\text { DAN_BK } \\
120\end{array}$ & $\begin{array}{lll}T \\
\end{array}$ & $T$ & $\mathrm{G}$ & $C$ & A & A & $C$ & $\mathrm{C}$ & $A$ & $\mathrm{~T}$ & $A$ & $T$ & $\mathrm{G}$ & $T$ & $\mathrm{G}$ & A & $\mathrm{C}$ \\
\hline $\begin{array}{c}\text { Europ } \\
\mathrm{e}\end{array}$ & Danes & $\begin{array}{c}\text { DAN_BK } \\
120\end{array}$ & $\mathrm{~T}$ & $T$ & $C$ & $C$ & $A$ & A & $C$ & $C$ & $A$ & $T$ & A & $T$ & $\mathrm{G}$ & $\mathrm{T}$ & $\mathrm{G}$ & $\bar{A}$ & $\mathrm{C}$ \\
\hline $\begin{array}{c}\text { Europ } \\
\mathrm{e}\end{array}$ & Danes & $\begin{array}{c}\text { DAN_BK } \\
124 \\
\end{array}$ & $\begin{array}{lll}T & \end{array}$ & $T$ & $\mathrm{G}$ & $\mathrm{C}$ & $\bar{A}$ & A & C & $C$ & $A$ & $T$ & $A$ & $T$ & $\mathrm{G}$ & $T$ & $\mathrm{G}$ & A & $\mathrm{C}$ \\
\hline $\begin{array}{c}\text { Europ } \\
\mathrm{e}\end{array}$ & Danes & $\begin{array}{c}\text { DAN_BK } \\
124\end{array}$ & $\mathrm{~T}$ & $T$ & $\mathrm{G}$ & $C$ & $\bar{A}$ & $\bar{A}$ & C & $C$ & $A$ & $T$ & $A$ & $T$ & $\bar{G}$ & $T$ & $G$ & $A$ & C \\
\hline $\begin{array}{c}\text { Europ } \\
\mathrm{e}\end{array}$ & Danes & $\begin{array}{c}\text { DAN_BK } \\
127\end{array}$ & $\mathrm{~T}$ & $T$ & $G$ & C & $\bar{A}$ & $\bar{A}$ & C & $C$ & $\bar{A}$ & $\mathrm{~T}$ & $A$ & $T$ & $G$ & $\mathrm{~T}$ & $G$ & $\bar{A}$ & C \\
\hline $\begin{array}{c}\text { Europ } \\
\mathrm{e}\end{array}$ & Danes & $\begin{array}{c}\text { DAN_BK } \\
127\end{array}$ & $\mathrm{~T}$ & 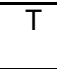 & $\mathrm{G}$ & $C$ & $A$ & $A$ & $C$ & $\mathrm{C}$ & $A$ & $\bar{T}$ & $A$ & $\mathrm{~T}$ & $\mathrm{G}$ & $\mathrm{T}$ & $\mathrm{G}$ & $A$ & $\mathrm{C}$ \\
\hline $\begin{array}{c}\text { Europ } \\
\mathrm{e}\end{array}$ & Danes & $\begin{array}{c}\text { DAN_BK } \\
141\end{array}$ & $\mathrm{~T}$ & $T$ & $\mathrm{G}$ & $C$ & $\bar{A}$ & $\bar{A}$ & $C$ & $\mathrm{C}$ & $A$ & $T$ & $A$ & $\bar{T}$ & $T$ & $T$ & $\mathrm{G}$ & A & C \\
\hline $\begin{array}{c}\text { Europ } \\
\mathrm{e}\end{array}$ & Danes & $\begin{array}{c}\text { DAN_BK } \\
141\end{array}$ & $\mathrm{~T}$ & $T$ & $\mathrm{G}$ & C & $G$ & $\bar{A}$ & $C$ & $\mathrm{C}$ & $A$ & $\mathrm{~T}$ & $\bar{A}$ & $T$ & $T$ & $\mathrm{~T}$ & $\mathrm{G}$ & C & C \\
\hline $\begin{array}{c}\text { Europ } \\
\mathrm{e}\end{array}$ & Danes & $\begin{array}{c}\text { DAN_BK } \\
144\end{array}$ & $\mathrm{~T}$ & $T$ & $\mathrm{G}$ & $C$ & $A$ & $A$ & $C$ & $\mathrm{C}$ & $A$ & $T$ & $A$ & $\mathrm{~T}$ & $\mathrm{G}$ & $\mathrm{T}$ & $\mathrm{G}$ & $A$ & $\mathrm{C}$ \\
\hline $\begin{array}{c}\text { Europ } \\
\mathrm{e}\end{array}$ & Danes & $\begin{array}{c}\text { DAN_BK } \\
144 \\
\end{array}$ & $\mathrm{~T}$ & $T$ & $C$ & $C$ & $A$ & $A$ & $C$ & $\mathrm{C}$ & $A$ & $T$ & $A$ & $T$ & $\mathrm{G}$ & $T$ & $\mathrm{G}$ & $A$ & $C$ \\
\hline $\begin{array}{c}\text { Europ } \\
\mathrm{e}\end{array}$ & Danes & $\begin{array}{c}\text { DAN_BK } \\
146\end{array}$ & $\mathrm{~T}$ & $T$ & $\mathrm{G}$ & $C$ & $A$ & A & C & C & $A$ & $T$ & $A$ & $T$ & $\mathrm{G}$ & $\mathrm{T}$ & $G$ & A & C \\
\hline $\begin{array}{c}\text { Europ } \\
\mathrm{e}\end{array}$ & Danes & $\begin{array}{c}\text { DAN_BK } \\
146\end{array}$ & $\mathrm{~T}$ & $T$ & $\mathrm{G}$ & $C$ & $A$ & $A$ & $C$ & $\mathrm{C}$ & A & $T$ & $A$ & $T$ & $\mathrm{~T}$ & $T$ & $\mathrm{G}$ & $A$ & $\mathrm{C}$ \\
\hline $\begin{array}{c}\text { Europ } \\
\mathrm{e}\end{array}$ & Danes & $\begin{array}{c}\text { DAN_BK } \\
153 \\
\end{array}$ & $\begin{array}{lll}T & \end{array}$ & $T$ & $\mathrm{G}$ & $C$ & $G$ & A & $C$ & $\mathrm{C}$ & $A$ & $\mathrm{~T}$ & $A$ & $T$ & $T$ & $T$ & $\mathrm{G}$ & A & C \\
\hline $\begin{array}{c}\text { Europ } \\
\mathrm{e}\end{array}$ & Danes & $\begin{array}{c}\text { DAN_BK } \\
153\end{array}$ & $\mathrm{~T}$ & $T$ & $\mathrm{G}$ & $C$ & $G$ & A & C & C & A & $T$ & $A$ & $T$ & $T$ & $\mathrm{~T}$ & $G$ & A & C \\
\hline $\begin{array}{c}\text { Europ } \\
\mathrm{e}\end{array}$ & Danes & $\begin{array}{c}\text { DAN_BK } \\
157\end{array}$ & $T$ & $\bar{T}$ & $\mathrm{G}$ & $\mathrm{C}$ & $\bar{A}$ & $\bar{A}$ & C & $\mathrm{C}$ & $A$ & $\mathrm{~T}$ & $A$ & $\mathrm{~T}$ & $\bar{G}$ & $\bar{T}$ & $\mathrm{G}$ & $\bar{A}$ & C \\
\hline Europ & Danes & DAN_BK & $\begin{array}{c}\mathrm{T} \\
\end{array}$ & $T$ & $G$ & $\mathrm{C}$ & $A$ & $A$ & C & $\mathrm{C}$ & $A$ & $T$ & $A$ & $T$ & $G$ & $T$ & $G$ & $A$ & $\mathrm{C}$ \\
\hline
\end{tabular}

John Wiley 13 Sons, Inc. 


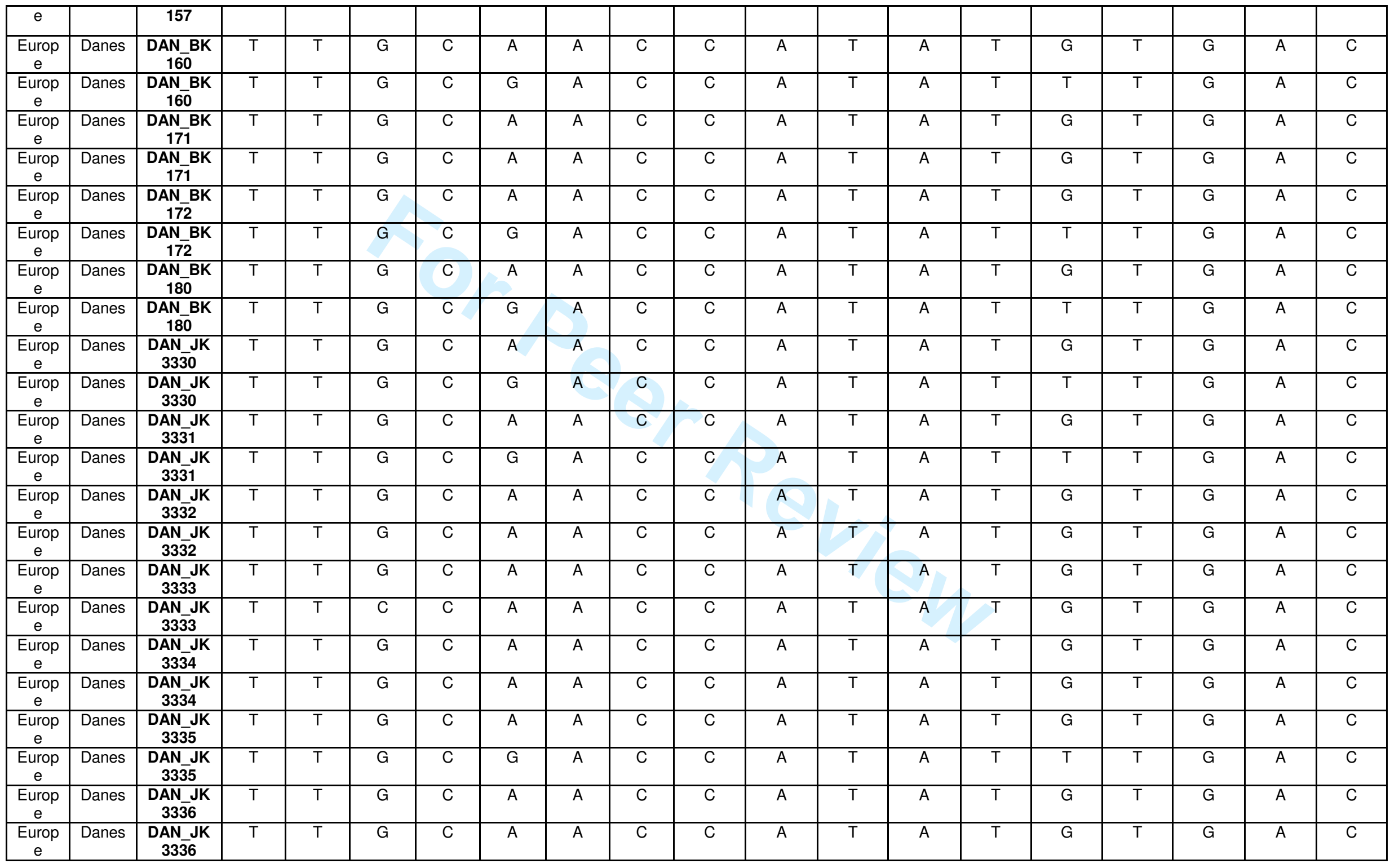

John Wiley 14 Sons, Inc. 
Supp. Table S2. Genotype information per individual of the TLR2 non-synonymous variants

\begin{tabular}{|c|c|c|c|c|c|c|c|c|c|}
\hline & & & SNP & SNP & SNP & SNP & SNP & SNP & SNP \\
\hline Continent & Population & ID & c. $265 A>G$ & c. $1232 C>T$ & c. $1339 \mathrm{C}>\mathrm{T}$ & c.1712G>A & c. $1892 C>A$ & c. $1906 A>C$ & c. $2258 \mathrm{G}>\mathrm{A}$ \\
\hline AFRICA & Chagga & CHA_JK4125 & $\mathrm{A}$ & $\mathrm{C}$ & $\mathrm{C}$ & G & $\mathrm{C}$ & $\mathrm{A}$ & G \\
\hline AFRICA & Chagga & CHA_JK4125 & $\mathrm{A}$ & $\mathrm{C}$ & $\mathrm{C}$ & G & $\mathrm{C}$ & $\mathrm{A}$ & G \\
\hline AFRICA & Chagga & CHA_JK4126 & $\mathrm{A}$ & $\mathrm{C}$ & $\mathrm{C}$ & $\mathrm{G}$ & $\mathrm{C}$ & $\bar{A}$ & G \\
\hline AFRICA & Chagga & CHA_JK4126 & $\mathrm{A}$ & $\mathrm{C}$ & $\mathrm{C}$ & G & $\mathrm{C}$ & $\mathrm{A}$ & G \\
\hline AFRICA & Chagga & CHA_JK4127 & $\mathrm{A}$ & $\mathrm{C}$ & $\mathrm{C}$ & $\bar{G}$ & $\mathrm{C}$ & $\bar{A}$ & $\bar{G}$ \\
\hline AFRICA & Chagga & CHA JK4127 & A & $\mathrm{C}$ & $\mathrm{C}$ & $\mathrm{G}$ & $\mathrm{C}$ & $\mathrm{A}$ & $\mathrm{G}$ \\
\hline AFRICA & Chagga & CHA_JK4128 & $\overline{\mathrm{A}}$ & $\mathrm{C}$ & $\mathrm{C}$ & G & $\mathrm{C}$ & $\overline{\mathrm{A}}$ & $\bar{G}$ \\
\hline AFRICA & Chagga & CHA_JK4128 & $\mathrm{A}$ & $\mathrm{C}$ & $\mathrm{C}$ & $\bar{G}$ & $\mathrm{C}$ & $\bar{A}$ & $\bar{G}$ \\
\hline AFRICA & Chagga & CHA_JK4129 & $\mathrm{A}$ & $\mathrm{C}$ & $\mathrm{C}$ & G & $\mathrm{C}$ & A & G \\
\hline AFRICA & Chagga & CHA_JK4129 & $\mathrm{A}$ & $\mathrm{C}$ & $\mathrm{C}$ & G & $\mathrm{C}$ & $\mathrm{A}$ & G \\
\hline AFRICA & Chagga & CHA_JK4130 & $\mathrm{A}$ & $\mathrm{C}$ & $\mathrm{C}$ & G & $\mathrm{C}$ & A & G \\
\hline AFRICA & Chagga & CHA_JK4130 & $\mathrm{A}$ & $\mathrm{C}$ & $\mathrm{C}$ & $\bar{G}$ & $\mathrm{C}$ & $\mathrm{A}$ & G \\
\hline AFRICA & Chagga & CHA_JK4131 & $\mathrm{A}$ & $\mathrm{C}$ & $\mathrm{C}$ & G & $\mathrm{C}$ & $\mathrm{A}$ & G \\
\hline AFRICA & Chagga & CHA_JK4131 & $\mathrm{A}$ & $\mathrm{C}$ & $\mathrm{C}$ & $\bar{G}$ & $\mathrm{C}$ & $\mathrm{C}$ & $\bar{G}$ \\
\hline AFRICA & Chagga & CHA_JK4132 & $\mathrm{A}$ & $\mathrm{C}$ & $\mathrm{C}$ & G & $\mathrm{C}$ & A & G \\
\hline AFRICA & Chagga & CHA_JK4132 & $\bar{A}$ & $\mathrm{C}$ & $\mathrm{C}$ & $\bar{G}$ & $\mathrm{C}$ & $\bar{A}$ & $\bar{G}$ \\
\hline AFRICA & Chagga & CHA_JK4133 & $\overline{\mathrm{A}}$ & $\mathrm{C}$ & $\mathrm{C}$ & $\bar{Y}$ & $\mathrm{C}$ & $\overline{\mathrm{A}}$ & $\bar{G}$ \\
\hline AFRICA & Chagga & CHA_JK4133 & $\mathrm{A}$ & $\mathrm{C}$ & $\mathrm{C}$ & $\bar{G}$ & $\mathrm{C}$ & $\mathrm{A}$ & G \\
\hline AFRICA & Chagga & CHA_JK4134 & $\mathrm{A}$ & $\mathrm{C}$ & $\mathrm{C}$ & G & $\mathrm{C}$ & $\mathrm{A}$ & G \\
\hline AFRICA & Chagga & CHA_JK4134 & $\mathrm{A}$ & $\mathrm{C}$ & $\mathrm{C}$ & G & $\mathrm{C}$ & $\mathrm{A}$ & G \\
\hline AFRICA & Chagga & CHA_JK4135 & $\bar{A}$ & $\mathrm{C}$ & $\mathrm{C}$ & $\bar{G}$ & $\mathrm{C}$ & $\mathrm{A}$ & G \\
\hline AFRICA & Chagga & CHA_JK4135 & A & $\mathrm{C}$ & $\mathrm{C}$ & $\mathrm{G}$ & $\mathrm{C}$ & $\mathrm{A}$ & G \\
\hline AFRICA & Chagga & CHA_JK4136 & $\bar{A}$ & $\mathrm{C}$ & $\mathrm{C}$ & $\bar{A}$ & $\mathrm{C}$ & $\overline{\mathrm{A}}$ & G \\
\hline AFRICA & Chagga & CHA JK4136 & $\mathrm{A}$ & $\mathrm{C}$ & $\mathrm{C}$ & G & $\mathrm{C}$ & $\mathrm{A}$ & $\mathrm{G}$ \\
\hline
\end{tabular}

John Wiley 15 Sons, Inc. 


\begin{tabular}{|c|c|c|c|c|c|c|c|c|c|}
\hline AFRICA & Chagga & CHA_JK4137 & $\mathrm{A}$ & $\mathrm{C}$ & $\mathrm{C}$ & $\mathrm{G}$ & $\mathrm{C}$ & $\mathrm{A}$ & $\bar{G}$ \\
\hline AFRICA & Chagga & CHA_JK4137 & $\mathrm{A}$ & $\mathrm{C}$ & $\mathrm{C}$ & $\mathrm{G}$ & $\mathrm{C}$ & A & $\mathrm{G}$ \\
\hline AFRICA & Chagga & CHA_JK4138 & $\mathrm{A}$ & $\bar{C}$ & $\mathrm{C}$ & $\mathrm{G}$ & $\mathrm{C}$ & $\overline{\mathrm{A}}$ & $\bar{G}$ \\
\hline AFRICA & Chagga & CHA_JK4138 & $\mathrm{A}$ & $\mathrm{C}$ & $\mathrm{C}$ & $\mathrm{G}$ & $\mathrm{C}$ & $\mathrm{A}$ & $\mathrm{G}$ \\
\hline AFRICA & Chagga & CHA_JK4139 & $\mathrm{A}$ & $\bar{C}$ & $\mathrm{C}$ & $\mathrm{G}$ & $\mathrm{C}$ & $\bar{A}$ & $\mathrm{G}$ \\
\hline AFRICA & Chagga & CHA_JK4139 & $\mathrm{A}$ & $\mathrm{C}$ & $\mathrm{C}$ & $\mathrm{G}$ & $\mathrm{C}$ & $\mathrm{A}$ & $\bar{G}$ \\
\hline AFRICA & Chagga & CHA_JK4140 & $\mathrm{A}$ & $\mathrm{C}$ & $\mathrm{C}$ & $\mathrm{G}$ & $\mathrm{C}$ & $\mathrm{A}$ & $\bar{G}$ \\
\hline AFRICA & Chagga & CHA_JK4140 & $\mathrm{A}$ & $\mathrm{C}$ & $\mathrm{C}$ & $\mathrm{G}$ & $\mathrm{C}$ & $\overline{\mathrm{A}}$ & $\bar{G}$ \\
\hline AFRICA & Chagga & CHA_JK4141 & $\mathrm{A}$ & $\bar{C}$ & $\bar{C}$ & $\mathrm{G}$ & $\mathrm{C}$ & $\overline{\mathrm{A}}$ & $\bar{G}$ \\
\hline AFRICA & Chagga & CHA_JK4141 & $\mathrm{A}$ & $\mathrm{C}$ & $\bar{C}$ & $\mathrm{G}$ & $\mathrm{C}$ & $\overline{\mathrm{A}}$ & $\bar{G}$ \\
\hline AFRICA & Chagga & CHA_JK4142 & $\mathrm{A}$ & $\mathrm{C}$ & $\mathrm{C}$ & $\mathrm{G}$ & $\mathrm{C}$ & $\mathrm{A}$ & $\mathrm{G}$ \\
\hline AFRICA & Chagga & CHA_JK4142 & $\bar{A}$ & 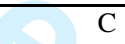 & $\mathrm{C}$ & $\mathrm{G}$ & $\mathrm{C}$ & $\overline{\mathrm{A}}$ & $\bar{G}$ \\
\hline AFRICA & Chagga & CHA_JK4143 & $\mathrm{A}$ & 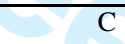 & $\mathrm{C}$ & $\mathrm{G}$ & $\mathrm{C}$ & $\bar{A}$ & $\mathrm{G}$ \\
\hline AFRICA & Chagga & CHA_JK4143 & $\mathrm{A}$ & $\mathrm{C}$ & $\mathrm{C}$ & $\mathrm{G}$ & $\mathrm{C}$ & $\bar{A}$ & $\bar{G}$ \\
\hline AFRICA & Chagga & CHA_JK4144 & $\mathrm{A}$ & $\mathrm{C}$ & $\mathrm{C}$ & $\mathrm{G}$ & $\mathrm{C}$ & $\mathrm{A}$ & $\mathrm{G}$ \\
\hline AFRICA & Chagga & CHA_JK4144 & $\mathrm{A}$ & $\mathrm{C}$ & $\mathrm{C}$ & $\mathrm{G}$ & $\mathrm{C}$ & $\mathrm{A}$ & $\bar{G}$ \\
\hline AFRICA & Chagga & CHA_JK4145 & $\mathrm{A}$ & $\mathrm{C}$ & $\mathrm{C}$ & $\mathrm{G}$ & $\mathrm{C}$ & $\mathrm{A}$ & $\bar{G}$ \\
\hline AFRICA & Chagga & CHA_JK4145 & $\mathrm{A}$ & $\mathrm{C}$ & $\mathrm{C}$ & $\mathrm{G}$ & $\mathrm{C}$ & $\bar{A}$ & $\mathrm{G}$ \\
\hline AFRICA & Chagga & CHA_JK4146 & $\mathrm{A}$ & $\bar{C}$ & $\bar{C}$ & $\mathrm{G}$ & $\mathrm{C}$ & $\mathrm{A}$ & $\bar{G}$ \\
\hline AFRICA & Chagga & CHA_JK4146 & $\mathrm{A}$ & $\mathrm{C}$ & $\mathrm{C}$ & $\mathrm{G}$ & $\mathrm{C}$ & $\mathrm{A}$ & $\mathrm{G}$ \\
\hline AFRICA & Chagga & CHA_JK4147 & $\mathrm{A}$ & $\bar{C}$ & $\mathrm{C}$ & $\mathrm{G}$ & $\mathrm{C}$ & $\mathrm{A}$ & $\bar{G}$ \\
\hline AFRICA & Chagga & CHA_JK4147 & $\mathrm{A}$ & $\mathrm{C}$ & $\mathrm{C}$ & $\mathrm{G}$ & $\mathrm{C}$ & $\mathrm{A}$ & $\mathrm{G}$ \\
\hline AFRICA & Chagga & CHA_JK4148 & $\mathrm{A}$ & $\mathrm{C}$ & $\bar{C}$ & $\mathrm{G}$ & $\mathrm{C}$ & $\bar{A}$ & $\mathrm{G}$ \\
\hline AFRICA & Chagga & CHA_JK4148 & $\mathrm{A}$ & $\mathrm{C}$ & $\mathrm{C}$ & $\mathrm{G}$ & $\mathrm{C}$ & $\mathrm{A}$ & $\mathrm{G}$ \\
\hline AFRICA & Chagga & CHA_JK4149 & $\mathrm{A}$ & $\mathrm{C}$ & $\mathrm{C}$ & $\mathrm{G}$ & $\mathrm{C}$ & $\mathrm{A}$ & $\mathrm{G}$ \\
\hline AFRICA & Chagga & CHA_JK4149 & $\mathrm{A}$ & $\mathrm{C}$ & $\mathrm{C}$ & $\mathrm{G}$ & $\mathrm{C}$ & $\bar{A}$ & $\bar{G}$ \\
\hline AFRICA & Chagga & CHA_JK4150 & $\mathrm{A}$ & $\mathrm{C}$ & $\mathrm{C}$ & $\mathrm{G}$ & $\mathrm{C}$ & $\mathrm{A}$ & $\bar{G}$ \\
\hline AFRICA & Chagga & CHA_JK4150 & $\mathrm{A}$ & $\mathrm{C}$ & $\mathrm{C}$ & $\mathrm{G}$ & $\mathrm{C}$ & $\mathrm{A}$ & $\mathrm{G}$ \\
\hline
\end{tabular}

John Wiley 16 Sons, Inc. 


\begin{tabular}{|c|c|c|c|c|c|c|c|c|c|}
\hline AFRICA & Chagga & CHA_JK4151 & $\bar{A}$ & $\mathrm{C}$ & $\overline{\mathrm{C}}$ & $\mathrm{G}$ & $\mathrm{C}$ & $\mathrm{A}$ & $\overline{\mathrm{G}}$ \\
\hline AFRICA & Chagga & CHA_JK4151 & $\mathrm{A}$ & $\mathrm{C}$ & $\mathrm{C}$ & $\mathrm{G}$ & $\mathrm{C}$ & A & $\mathrm{G}$ \\
\hline AFRICA & Chagga & CHA_JK4152 & $\overline{\mathrm{A}}$ & $\mathrm{C}$ & $\overline{\mathrm{C}}$ & $\mathrm{G}$ & $\bar{C}$ & $\overline{\mathrm{A}}$ & $\overline{\mathrm{G}}$ \\
\hline AFRICA & Chagga & CHA_JK4152 & $\overline{\mathrm{A}}$ & $\mathrm{C}$ & $\bar{C}$ & G & $\mathrm{C}$ & A & $\bar{G}$ \\
\hline AFRICA & Chagga & CHA_JK4153 & $\overline{\mathrm{A}}$ & $\mathrm{C}$ & $\overline{\mathrm{C}}$ & $\bar{G}$ & $\bar{C}$ & $\overline{\mathrm{A}}$ & $\overline{\mathrm{G}}$ \\
\hline AFRICA & Chagga & CHA_JK4153 & $\mathrm{A}$ & $\mathrm{C}$ & $\mathrm{C}$ & $\mathrm{G}$ & $\mathrm{C}$ & $\mathrm{A}$ & $\mathrm{G}$ \\
\hline AFRICA & Chagga & CHA_JK4154 & $\overline{\mathrm{A}}$ & $\mathrm{C}$ & $\mathrm{C}$ & G & $\mathrm{C}$ & $\overline{\mathrm{A}}$ & $\mathrm{G}$ \\
\hline AFRICA & Chagga & CHA_JK4154 & $\overline{\mathrm{A}}$ & $\mathrm{C}$ & $\mathrm{C}$ & $\mathrm{G}$ & $\mathrm{C}$ & $\bar{A}$ & $\bar{G}$ \\
\hline AFRICA & Chagga & CHA_JK4155 & $\overline{\mathrm{A}}$ & $\mathrm{C}$ & $\overline{\mathrm{C}}$ & $\mathrm{G}$ & $\mathrm{C}$ & $\overline{\mathrm{A}}$ & $\overline{\mathrm{G}}$ \\
\hline AFRICA & Chagga & CHA_JK4155 & $\overline{\mathrm{A}}$ & $\mathrm{C}$ & $\overline{\mathrm{C}}$ & $\mathrm{G}$ & $\mathrm{C}$ & $\overline{\mathrm{A}}$ & $\overline{\mathrm{G}}$ \\
\hline AFRICA & Chagga & CHA_JK4156 & $\overline{\mathrm{A}}$ & $\mathrm{C}$ & $\mathrm{C}$ & G & $\mathrm{C}$ & A & $\mathrm{G}$ \\
\hline AFRICA & Chagga & CHA_JK4156 & $\overline{\mathrm{A}}$ & $\mathrm{C}$ & $\bar{C}$ & $\bar{G}$ & $\mathrm{C}$ & $\bar{A}$ & $\bar{G}$ \\
\hline AFRICA & Yoruba & YRB_JK3878 & $\mathrm{A}$ & $\mathrm{C}$ & $\mathrm{C}$ & $\mathrm{G}$ & $\mathrm{C}$ & $\mathrm{A}$ & $\mathrm{G}$ \\
\hline AFRICA & Yoruba & YRB_JK3878 & $\overline{\mathrm{A}}$ & $\mathrm{C}$ & $\mathrm{C}$ & $\bar{G}$ & $\mathrm{C}$ & $\bar{A}$ & $\bar{G}$ \\
\hline AFRICA & Yoruba & YRB_JK3879 & $\mathrm{A}$ & $\mathrm{C}$ & $\mathrm{C}$ & $\mathrm{G}$ & $\mathrm{C}$ & $\mathrm{A}$ & $\mathrm{G}$ \\
\hline AFRICA & Yoruba & YRB_JK3879 & $\mathrm{A}$ & $\mathrm{C}$ & $\bar{C}$ & $\mathrm{G}$ & $\mathrm{C}$ & $\mathrm{A}$ & $\mathrm{G}$ \\
\hline AFRICA & Yoruba & YRB_JK3880 & $\mathrm{A}$ & $\mathrm{C}$ & $\mathrm{C}$ & $\bar{G}$ & $\mathrm{C}$ & $\bar{A}$ & $\bar{G}$ \\
\hline AFRICA & Yoruba & YRB_JK3880 & $\mathrm{A}$ & $\mathrm{C}$ & $\overline{\mathrm{C}}$ & $\mathrm{G}$ & $\bar{C}$ & $\overline{\mathrm{A}}$ & $\overline{\mathrm{G}}$ \\
\hline AFRICA & Yoruba & YRB_JK3881 & $\mathrm{A}$ & $\mathrm{C}$ & $\mathrm{C}$ & $\bar{G}$ & $\mathrm{C}$ & $\bar{A}$ & $\bar{G}$ \\
\hline AFRICA & Yoruba & YRB_JK3881 & $\mathrm{A}$ & $\mathrm{C}$ & $\bar{C}$ & G & $\mathrm{C}$ & A & $\bar{G}$ \\
\hline AFRICA & Yoruba & YRB_JK3882 & $\mathrm{A}$ & $\mathrm{C}$ & $\bar{C}$ & $\bar{G}$ & $\mathrm{C}$ & $\bar{A}$ & $\bar{G}$ \\
\hline AFRICA & Yoruba & YRB_JK3882 & $\mathrm{A}$ & $\mathrm{C}$ & $\mathrm{C}$ & $\mathrm{G}$ & $\mathrm{C}$ & $\mathrm{A}$ & $\mathrm{G}$ \\
\hline AFRICA & Yoruba & YRB_JK3883 & $\mathrm{A}$ & $\bar{C}$ & $\overline{\mathrm{C}}$ & $\bar{G}$ & $\bar{C}$ & $\overline{\mathrm{A}}$ & $\overline{\mathrm{G}}$ \\
\hline AFRICA & Yoruba & YRB_JK3883 & $\mathrm{A}$ & $\mathrm{C}$ & $\mathrm{C}$ & G & $\mathrm{C}$ & A & $\mathrm{G}$ \\
\hline AFRICA & Yoruba & YRB_JK3884 & $\mathrm{A}$ & $\mathrm{C}$ & $\overline{\mathrm{C}}$ & G & $\mathrm{C}$ & $\mathrm{A}$ & $\bar{G}$ \\
\hline AFRICA & Yoruba & YRB_JK3884 & $\mathrm{A}$ & $\mathrm{C}$ & $\overline{\mathrm{C}}$ & $\mathrm{G}$ & $\mathrm{C}$ & $\overline{\mathrm{A}}$ & $\overline{\mathrm{G}}$ \\
\hline AFRICA & Yoruba & YRB_JK3885 & $\mathrm{A}$ & $\mathrm{C}$ & $\mathrm{C}$ & $\mathrm{G}$ & $\mathrm{C}$ & $\mathrm{A}$ & $\bar{G}$ \\
\hline AFRICA & Yoruba & YRB_JK3885 & $\mathrm{A}$ & $\bar{C}$ & $\mathrm{C}$ & $\mathrm{G}$ & $\mathrm{C}$ & $\mathrm{A}$ & $\mathrm{G}$ \\
\hline
\end{tabular}

\author{
John Wiley \& Sons, Inc.
}




\begin{tabular}{|c|c|c|c|c|c|c|c|c|c|}
\hline AFRICA & Yoruba & YRB_JK3886 & $\mathrm{A}$ & $\mathrm{C}$ & $\mathrm{C}$ & $\bar{G}$ & $\bar{C}$ & $\mathrm{~A}$ & $\bar{G}$ \\
\hline AFRICA & Yoruba & YRB_JK3886 & $\mathrm{A}$ & $\mathrm{C}$ & $\mathrm{C}$ & $\mathrm{G}$ & $\mathrm{C}$ & $\mathrm{A}$ & $\mathrm{G}$ \\
\hline AFRICA & Yoruba & YRB_JK3887 & $\mathrm{A}$ & $\bar{C}$ & $\bar{C}$ & $\mathrm{G}$ & $\mathrm{C}$ & $\overline{\mathrm{A}}$ & $\bar{G}$ \\
\hline AFRICA & Yoruba & YRB_JK3887 & $\mathrm{A}$ & $\mathrm{C}$ & $\mathrm{C}$ & $\mathrm{G}$ & $\mathrm{C}$ & A & $\mathrm{G}$ \\
\hline AFRICA & Yoruba & YRB_JK3888 & $\mathrm{A}$ & $\bar{C}$ & $\mathrm{C}$ & $\mathrm{G}$ & $\mathrm{C}$ & $\overline{\mathrm{A}}$ & $\bar{G}$ \\
\hline AFRICA & Yoruba & YRB_JK3888 & $\mathrm{A}$ & $\mathrm{C}$ & $\mathrm{C}$ & $\mathrm{G}$ & $\mathrm{C}$ & $\bar{A}$ & $\mathrm{G}$ \\
\hline AFRICA & Yoruba & YRB_JK3889 & $\mathrm{A}$ & $\mathrm{C}$ & $\mathrm{C}$ & $\mathrm{G}$ & $\mathrm{C}$ & $\bar{A}$ & $\mathrm{G}$ \\
\hline AFRICA & Yoruba & YRB_JK3889 & $\mathrm{A}$ & $\bar{C}$ & $\mathrm{C}$ & $\mathrm{G}$ & $\mathrm{C}$ & $\bar{A}$ & $\bar{G}$ \\
\hline AFRICA & Yoruba & YRB_JK3891 & $\mathrm{A}$ & $\mathrm{C}$ & $\bar{C}$ & $\mathrm{G}$ & $\mathrm{C}$ & $\mathrm{A}$ & $\bar{G}$ \\
\hline AFRICA & Yoruba & YRB_JK3891 & $\mathrm{A}$ & $\mathrm{C}$ & $\bar{C}$ & $\mathrm{G}$ & $\mathrm{C}$ & $\overline{\mathrm{A}}$ & $\mathrm{G}$ \\
\hline AFRICA & Yoruba & YRB_JK3892 & $\mathrm{A}$ & $\mathrm{C}$ & $\mathrm{C}$ & $\mathrm{G}$ & $\mathrm{C}$ & $\mathrm{A}$ & $\mathrm{G}$ \\
\hline AFRICA & Yoruba & YRB_JK3892 & $\mathrm{A}$ & 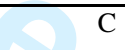 & $\mathrm{C}$ & $\mathrm{G}$ & $\mathrm{C}$ & $\bar{A}$ & $\bar{G}$ \\
\hline AFRICA & Yoruba & YRB_JK3893 & $\mathrm{A}$ & $\mathrm{C}$ & $\mathrm{C}$ & $\mathrm{G}$ & $\mathrm{C}$ & A & $\mathrm{G}$ \\
\hline AFRICA & Yoruba & YRB_JK3893 & $\mathrm{A}$ & $\bar{C}$ & $\mathrm{C}$ & $\mathrm{G}$ & $\mathrm{C}$ & $\mathrm{A}$ & $\bar{G}$ \\
\hline AFRICA & Yoruba & YRB_JK3894 & $\mathrm{A}$ & $\mathrm{C}$ & $\mathrm{C}$ & $\mathrm{G}$ & $\mathrm{C}$ & $\mathrm{A}$ & $\mathrm{G}$ \\
\hline AFRICA & Yoruba & YRB_JK3894 & $\mathrm{A}$ & $\mathrm{C}$ & $\mathrm{C}$ & $\mathrm{G}$ & $\mathrm{C}$ & $\mathrm{A}$ & $\mathrm{G}$ \\
\hline AFRICA & Yoruba & YRB_JK3845 & $\mathrm{A}$ & $\mathrm{C}$ & $\mathrm{C}$ & $\mathrm{G}$ & $\mathrm{C}$ & $\bar{A}$ & $\mathrm{G}$ \\
\hline AFRICA & Yoruba & YRB_JK3845 & $\mathrm{A}$ & $\mathrm{C}$ & $\mathrm{C}$ & $\mathrm{G}$ & $\mathrm{C}$ & $\mathrm{A}$ & $\bar{G}$ \\
\hline AFRICA & Yoruba & YRB_JK3846 & $\mathrm{A}$ & $\mathrm{C}$ & $\mathrm{C}$ & $\mathrm{G}$ & $\mathrm{C}$ & $\mathrm{A}$ & $\bar{G}$ \\
\hline AFRICA & Yoruba & YRB_JK3846 & $\mathrm{A}$ & $\mathrm{C}$ & $\mathrm{C}$ & $\mathrm{G}$ & $\mathrm{C}$ & $\mathrm{A}$ & $\mathrm{G}$ \\
\hline AFRICA & Yoruba & YRB_JK3847 & $\mathrm{A}$ & $\mathrm{C}$ & $\mathrm{C}$ & $\mathrm{G}$ & $\mathrm{C}$ & $\mathrm{A}$ & $\bar{G}$ \\
\hline AFRICA & Yoruba & YRB_JK3847 & $\mathrm{A}$ & $\mathrm{C}$ & $\mathrm{C}$ & $\mathrm{G}$ & $\mathrm{C}$ & $\mathrm{A}$ & $\mathrm{G}$ \\
\hline AFRICA & Yoruba & YRB_JK3848 & $\mathrm{A}$ & $\mathrm{C}$ & $\mathrm{C}$ & $\mathrm{G}$ & $\mathrm{C}$ & $\mathrm{A}$ & $\bar{G}$ \\
\hline AFRICA & Yoruba & YRB_JK3848 & $\mathrm{A}$ & $\mathrm{C}$ & $\mathrm{C}$ & $\mathrm{G}$ & $\mathrm{C}$ & $\mathrm{A}$ & $\mathrm{G}$ \\
\hline AFRICA & Yoruba & YRB_JK3849 & $\mathrm{A}$ & $\mathrm{C}$ & $\mathrm{C}$ & $\mathrm{G}$ & $\mathrm{C}$ & $\mathrm{A}$ & $\mathrm{G}$ \\
\hline AFRICA & Yoruba & YRB_JK3849 & $\mathrm{A}$ & $\mathrm{C}$ & $\bar{C}$ & $\mathrm{G}$ & $\mathrm{C}$ & $\mathrm{A}$ & $\mathrm{G}$ \\
\hline AFRICA & Yoruba & YRB_JK3850 & $\mathrm{A}$ & $\mathrm{C}$ & $\mathrm{C}$ & $\mathrm{G}$ & $\mathrm{C}$ & $\mathrm{A}$ & $\bar{G}$ \\
\hline AFRICA & Yoruba & YRB_JK3850 & $\mathrm{A}$ & $\mathrm{C}$ & $\mathrm{C}$ & $\mathrm{G}$ & $\mathrm{C}$ & $\mathrm{A}$ & $\bar{G}$ \\
\hline
\end{tabular}

John Wiley 18 Sons, Inc. 


\begin{tabular}{|c|c|c|c|c|c|c|c|c|c|}
\hline AFRICA & Yoruba & YRB_JK3851 & $\overline{\mathrm{A}}$ & $\bar{C}$ & $\mathrm{C}$ & $\mathrm{G}$ & $\mathrm{C}$ & $\overline{\mathrm{A}}$ & $\bar{G}$ \\
\hline AFRICA & Yoruba & YRB_JK3851 & $\bar{A}$ & $\mathrm{C}$ & $\mathrm{C}$ & G & $\mathrm{C}$ & $\bar{A}$ & G \\
\hline AFRICA & Yoruba & YRB_JK3852 & $\overline{\mathrm{A}}$ & $\mathrm{C}$ & $\mathrm{C}$ & $\mathrm{G}$ & $\bar{C}$ & $\overline{\mathrm{A}}$ & $\bar{G}$ \\
\hline AFRICA & Yoruba & YRB_JK3852 & A & $\mathrm{C}$ & $\mathrm{C}$ & $\mathrm{G}$ & $\mathrm{C}$ & A & $\mathrm{G}$ \\
\hline AFRICA & Yoruba & YRB_JK3853 & $\bar{A}$ & $\mathrm{C}$ & $\mathrm{C}$ & $\bar{G}$ & $\bar{C}$ & $\overline{\mathrm{A}}$ & $\bar{G}$ \\
\hline AFRICA & Yoruba & YRB_JK3853 & A & $\mathrm{C}$ & $\mathrm{C}$ & $\mathrm{G}$ & $\mathrm{C}$ & A & $\mathrm{G}$ \\
\hline AFRICA & Yoruba & YRB_JK3854 & A & $\mathrm{C}$ & $\mathrm{C}$ & $\mathrm{G}$ & $\mathrm{C}$ & A & $\mathrm{G}$ \\
\hline AFRICA & Yoruba & YRB_JK3854 & A & $\mathrm{C}$ & $\mathrm{C}$ & $\mathrm{G}$ & $\mathrm{C}$ & A & $\mathrm{G}$ \\
\hline AFRICA & Yoruba & YRB_JK3855 & $\bar{A}$ & $\mathrm{C}$ & $\mathrm{C}$ & $\mathrm{G}$ & $\mathrm{C}$ & $\overline{\mathrm{A}}$ & $\bar{G}$ \\
\hline AFRICA & Yoruba & YRB_JK3855 & $\overline{\mathrm{A}}$ & $\bar{C}$ & $\mathrm{C}$ & $\mathrm{G}$ & $\mathrm{C}$ & $\overline{\mathrm{A}}$ & $\bar{G}$ \\
\hline AFRICA & Yoruba & YRB_JK3856 & A & $\mathrm{C}$ & $\mathrm{C}$ & G & $\mathrm{C}$ & A & G \\
\hline AFRICA & Yoruba & YRB_JK3856 & $\bar{A}$ & $\bar{C}$ & $\mathrm{C}$ & $\bar{G}$ & $\mathrm{C}$ & $\bar{A}$ & $\bar{G}$ \\
\hline AFRICA & Yoruba & YRB_JK3860 & $\mathrm{A}$ & +2 & $\mathrm{C}$ & $\mathrm{G}$ & $\mathrm{C}$ & $\mathrm{A}$ & $\mathrm{G}$ \\
\hline AFRICA & Yoruba & YRB_JK3860 & $\bar{A}$ & $\overline{\mathrm{C}}$ & $\mathrm{C}$ & $\bar{G}$ & $\mathrm{C}$ & $\overline{\mathrm{A}}$ & $\bar{G}$ \\
\hline AFRICA & Yoruba & YRB_JK3858 & $\mathrm{A}$ & $\bar{C}$ & $\mathrm{C}$ & $\mathrm{G}$ & $\mathrm{C}$ & A & $\mathrm{G}$ \\
\hline AFRICA & Yoruba & YRB_JK3858 & $\mathrm{A}$ & $\mathrm{C}$ & $\mathrm{C}$ & $\mathrm{G}$ & $\mathrm{C}$ & A & $\mathrm{G}$ \\
\hline AFRICA & Yoruba & YRB_JK3859 & $\bar{A}$ & $\bar{C}$ & $\mathrm{C}$ & $\bar{G}$ & $\mathrm{C}$ & $\bar{A}$ & $\bar{G}$ \\
\hline AFRICA & Yoruba & YRB_JK3859 & $\overline{\mathrm{A}}$ & $\mathrm{C}$ & $\mathrm{C}$ & $\mathrm{G}$ & $\bar{C}$ & $\overline{\mathrm{A}}$ & $\bar{G}$ \\
\hline East-Asia & China & CH_CH25 & $\bar{A}$ & $\bar{C}$ & $\mathrm{C}$ & $\bar{G}$ & $\mathrm{C}$ & $\bar{A}$ & $\mathrm{G}$ \\
\hline East-Asia & China & CH_CH25 & $\bar{A}$ & $\mathrm{C}$ & $\mathrm{C}$ & G & $\mathrm{C}$ & $\bar{A}$ & $\bar{G}$ \\
\hline East-Asia & China & CH_CH02 & $\bar{A}$ & $\bar{C}$ & $\mathrm{C}$ & $\bar{G}$ & $\mathrm{C}$ & $\bar{A}$ & $\bar{G}$ \\
\hline East-Asia & China & CH_CH02 & A & $\mathrm{C}$ & $\mathrm{C}$ & G & $\mathrm{C}$ & A & G \\
\hline East-Asia & China & CH_CHO3 & $\bar{A}$ & $\bar{C}$ & $\bar{C}$ & $\mathrm{G}$ & $\mathrm{C}$ & $\bar{A}$ & G \\
\hline East-Asia & China & $\mathrm{CH} \_\mathrm{CHO3}$ & A & $\mathrm{C}$ & $\mathrm{C}$ & G & $\mathrm{C}$ & A & G \\
\hline East-Asia & China & CH_CH04 & $\bar{A}$ & $\mathrm{C}$ & $\mathrm{C}$ & G & $\mathrm{C}$ & $\overline{\mathrm{A}}$ & G \\
\hline East-Asia & China & CH_CH04 & $\bar{A}$ & $\mathrm{C}$ & $\mathrm{C}$ & $\mathrm{G}$ & $\mathrm{C}$ & $\overline{\mathrm{A}}$ & $\bar{G}$ \\
\hline East-Asia & China & CH_CH05 & $\bar{A}$ & $\mathrm{C}$ & $\mathrm{C}$ & $\bar{G}$ & $\mathrm{C}$ & $\bar{A}$ & $\bar{G}$ \\
\hline East-Asia & China & CH_CH05 & $\bar{A}$ & $\mathrm{C}$ & $\mathrm{C}$ & $\mathrm{G}$ & $\mathrm{C}$ & $\bar{A}$ & $\mathrm{G}$ \\
\hline
\end{tabular}




\begin{tabular}{|c|c|c|c|c|c|c|c|c|c|}
\hline East-Asia & China & CH_CH06 & $\mathrm{A}$ & $\mathrm{C}$ & $\mathrm{C}$ & $\mathrm{G}$ & $\mathrm{C}$ & $\bar{A}$ & $\bar{G}$ \\
\hline East-Asia & China & CH_CH06 & $\mathrm{A}$ & $\mathrm{C}$ & $\mathrm{C}$ & $\mathrm{G}$ & $\mathrm{C}$ & $\mathrm{A}$ & $\mathrm{G}$ \\
\hline East-Asia & China & $\mathrm{CH} \_\mathrm{CHO7}$ & $\mathrm{A}$ & $\mathrm{C}$ & $\mathrm{C}$ & $\mathrm{G}$ & $\mathrm{C}$ & $\overline{\mathrm{A}}$ & $\bar{G}$ \\
\hline East-Asia & China & CH_CH07 & $\mathrm{A}$ & $\mathrm{C}$ & $\mathrm{C}$ & $\mathrm{G}$ & $\mathrm{C}$ & $\mathrm{A}$ & $\mathrm{G}$ \\
\hline East-Asia & China & CH_CH08 & $\mathrm{A}$ & $\bar{C}$ & $\mathrm{C}$ & $\mathrm{G}$ & $\mathrm{C}$ & $\bar{A}$ & $\mathrm{G}$ \\
\hline East-Asia & China & CH_CH08 & $\mathrm{A}$ & $\mathrm{C}$ & $\mathrm{C}$ & $\mathrm{G}$ & $\mathrm{C}$ & $\mathrm{A}$ & $\bar{G}$ \\
\hline East-Asia & China & CH_CH09 & $\mathrm{A}$ & $\mathrm{C}$ & $\mathrm{C}$ & $\mathrm{G}$ & $\mathrm{C}$ & $\bar{A}$ & $\mathrm{G}$ \\
\hline East-Asia & China & CH_CH09 & $\mathrm{A}$ & $\mathrm{C}$ & $\mathrm{C}$ & $\mathrm{G}$ & $\mathrm{C}$ & $\overline{\mathrm{A}}$ & $\bar{G}$ \\
\hline East-Asia & China & $\mathrm{CH} \_\mathrm{CH} 10$ & $\mathrm{~A}$ & $\mathrm{C}$ & $\mathrm{C}$ & $\mathrm{G}$ & $\mathrm{C}$ & $\mathrm{A}$ & $\mathrm{G}$ \\
\hline East-Asia & China & CH_CH10 & $\mathrm{A}$ & $\mathrm{C}$ & $\bar{C}$ & $\mathrm{G}$ & $\mathrm{C}$ & $\overline{\mathrm{A}}$ & $\mathrm{G}$ \\
\hline East-Asia & China & $\mathrm{CH} \_\mathrm{CH} 11$ & $\mathrm{~A}$ & $\mathrm{C}$ & $\mathrm{C}$ & $\mathrm{G}$ & $\mathrm{C}$ & $\mathrm{A}$ & $\mathrm{G}$ \\
\hline East-Asia & China & CH_CH11 & $\mathrm{A}$ & 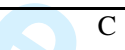 & $\mathrm{C}$ & $\mathrm{G}$ & $\mathrm{C}$ & $\bar{A}$ & $\mathrm{G}$ \\
\hline East-Asia & China & $\mathrm{CH} \_\mathrm{CH} 12$ & $\mathrm{~A}$ & 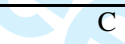 & $\mathrm{C}$ & $\mathrm{G}$ & $\mathrm{C}$ & $\bar{A}$ & $\mathrm{G}$ \\
\hline East-Asia & China & CH_CH12 & $\mathrm{A}$ & $\mathrm{C}$ & $\mathrm{C}$ & $\mathrm{G}$ & $\mathrm{C}$ & $\bar{A}$ & $\mathrm{G}$ \\
\hline East-Asia & China & $\mathrm{CH}$ CH13 & $\mathrm{A}$ & $\mathrm{C}$ & $\mathrm{C}$ & $\mathrm{G}$ & $\mathrm{C}$ & $\mathrm{A}$ & $\mathrm{G}$ \\
\hline East-Asia & China & CH_CH13 & $\mathrm{A}$ & $\mathrm{C}$ & $\mathrm{C}$ & $\mathrm{G}$ & $\mathrm{C}$ & $\mathrm{A}$ & $\mathrm{G}$ \\
\hline East-Asia & China & CH_CH14 & $\mathrm{A}$ & $\mathrm{C}$ & $\mathrm{C}$ & $\mathrm{G}$ & $\mathrm{C}$ & $\mathrm{A}$ & $\bar{G}$ \\
\hline East-Asia & China & CH_CH14 & $\mathrm{A}$ & $\mathrm{C}$ & $\mathrm{C}$ & $\mathrm{G}$ & $\mathrm{C}$ & $\mathrm{A}$ & $\bar{G}$ \\
\hline East-Asia & China & CH_CH15 & $\mathrm{A}$ & $\mathrm{C}$ & $\mathrm{C}$ & $\mathrm{G}$ & $\mathrm{C}$ & $\mathrm{A}$ & $\bar{G}$ \\
\hline East-Asia & China & CH_CH15 & $\mathrm{A}$ & $\mathrm{C}$ & $\mathrm{C}$ & $\mathrm{G}$ & $\mathrm{C}$ & $\mathrm{A}$ & $\mathrm{G}$ \\
\hline East-Asia & China & $\mathrm{CH} \_\mathrm{CH} 16$ & $\mathrm{~A}$ & $\bar{C}$ & $\mathrm{C}$ & $\mathrm{G}$ & $\mathrm{C}$ & $\mathrm{A}$ & $\bar{G}$ \\
\hline East-Asia & China & $\mathrm{CH} \_\mathrm{CH} 16$ & $\mathrm{~A}$ & $\mathrm{C}$ & $\mathrm{C}$ & $\mathrm{G}$ & $\mathrm{C}$ & $\mathrm{A}$ & $\mathrm{G}$ \\
\hline East-Asia & China & CH_CH17 & $\mathrm{A}$ & $\mathrm{C}$ & $\bar{C}$ & $\mathrm{G}$ & $\mathrm{C}$ & $\bar{A}$ & $\mathrm{G}$ \\
\hline East-Asia & China & CH_CH17 & $\mathrm{A}$ & $\mathrm{C}$ & $\mathrm{C}$ & $\mathrm{G}$ & $\mathrm{C}$ & $\mathrm{A}$ & $\mathrm{G}$ \\
\hline East-Asia & China & CH_CH18 & $\mathrm{A}$ & $\mathrm{C}$ & $\mathrm{C}$ & $\mathrm{G}$ & $\mathrm{C}$ & $\mathrm{A}$ & $\mathrm{G}$ \\
\hline East-Asia & China & $\mathrm{CH} \_\mathrm{CH} 18$ & $\mathrm{~A}$ & $\mathrm{C}$ & $\mathrm{C}$ & $\mathrm{G}$ & $\mathrm{C}$ & $\bar{A}$ & $\bar{G}$ \\
\hline East-Asia & China & CH_CH19 & $\mathrm{A}$ & $\mathrm{C}$ & $\mathrm{C}$ & $\mathrm{G}$ & $\mathrm{C}$ & $\mathrm{A}$ & $\mathrm{G}$ \\
\hline East-Asia & China & CH_CH19 & $\mathrm{A}$ & $\mathrm{C}$ & $\mathrm{C}$ & $\mathrm{G}$ & $\mathrm{C}$ & $\mathrm{A}$ & $\mathrm{G}$ \\
\hline
\end{tabular}

John Wiley ${ }^{2} \&$ Sons, Inc. 


\begin{tabular}{|c|c|c|c|c|c|c|c|c|c|}
\hline East-Asia & China & CH_CH2O & $\bar{A}$ & $\bar{C}$ & $\mathrm{C}$ & $\mathrm{G}$ & $\mathrm{C}$ & $\bar{A}$ & $\bar{G}$ \\
\hline East-Asia & China & $\mathrm{CH} \_\mathrm{CH} 20$ & $\mathrm{~A}$ & $\mathrm{C}$ & $\mathrm{C}$ & $\mathrm{G}$ & $\mathrm{C}$ & $\mathrm{A}$ & $\mathrm{G}$ \\
\hline East-Asia & China & $\mathrm{CH} \_\mathrm{CH} 21$ & $\overline{\mathrm{A}}$ & $\mathrm{C}$ & $\bar{C}$ & $\mathrm{G}$ & $\mathrm{C}$ & $\overline{\mathrm{A}}$ & $\overline{\mathrm{G}}$ \\
\hline East-Asia & China & $\mathrm{CH} \_\mathrm{CH} 21$ & A & $\mathrm{C}$ & C & G & $\mathrm{C}$ & A & $\mathrm{G}$ \\
\hline East-Asia & China & CH_CH22 & $\overline{\mathrm{A}}$ & $\mathrm{C}$ & $\bar{C}$ & $\mathrm{G}$ & $\mathrm{C}$ & $\bar{A}$ & $\overline{\mathrm{G}}$ \\
\hline East-Asia & China & CH_CH22 & $\bar{A}$ & $\mathrm{C}$ & $\mathrm{C}$ & $\mathrm{G}$ & $\mathrm{C}$ & $\overline{\mathrm{A}}$ & $\mathrm{G}$ \\
\hline East-Asia & China & CH_CH23 & $\bar{A}$ & $\mathrm{C}$ & $\mathrm{C}$ & $\mathrm{G}$ & $\mathrm{C}$ & $\overline{\mathrm{A}}$ & $\mathrm{G}$ \\
\hline East-Asia & China & $\mathrm{CH} \_\mathrm{CH} 23$ & $\overline{\mathrm{A}}$ & $\mathrm{C}$ & $\mathrm{C}$ & $\mathrm{G}$ & $\mathrm{C}$ & $\bar{A}$ & $\bar{G}$ \\
\hline East-Asia & China & CH_CH24 & $\bar{A}$ & $\mathrm{C}$ & $\mathrm{C}$ & $\mathrm{G}$ & $\mathrm{C}$ & $\overline{\mathrm{A}}$ & $\bar{G}$ \\
\hline East-Asia & China & CH_CH24 & $\mathrm{A}$ & $\mathrm{C}$ & $\mathrm{C}$ & $\mathrm{G}$ & $\mathrm{C}$ & $\overline{\mathrm{A}}$ & $\bar{G}$ \\
\hline East-Asia & Japanese & JPN_J36 & $\mathrm{A}$ & $\mathrm{C}$ & $\mathrm{C}$ & $\mathrm{G}$ & $\mathrm{C}$ & $\mathrm{A}$ & $\mathrm{G}$ \\
\hline East-Asia & Japanese & JPN_J36 & $\bar{A}$ & $\mathrm{C}$ & $\mathrm{C}$ & $\mathrm{G}$ & $\mathrm{C}$ & $\bar{A}$ & $\mathrm{G}$ \\
\hline East-Asia & Japanese & JPN_J37 & $\mathrm{A}$ & $\mathrm{C}$ & $\bar{C}$ & $\mathrm{G}$ & $\mathrm{C}$ & $\bar{A}$ & $\bar{G}$ \\
\hline East-Asia & Japanese & JPN_J37 & $\overline{\mathrm{A}}$ & $\mathrm{C}$ & $\bar{C}$ & $\mathrm{G}$ & $\mathrm{C}$ & $\bar{A}$ & $\overline{\mathrm{G}}$ \\
\hline East-Asia & Japanese & JPN_J38 & A & $\mathrm{C}$ & $\mathrm{C}$ & $\mathrm{G}$ & $\mathrm{C}$ & $\mathrm{A}$ & $\mathrm{G}$ \\
\hline East-Asia & Japanese & JPN_J38 & $\bar{A}$ & $\mathrm{C}$ & $\mathrm{C}$ & $\mathrm{G}$ & $\mathrm{C}$ & $\bar{A}$ & $\mathrm{G}$ \\
\hline East-Asia & Japanese & JPN_J39 & $\bar{A}$ & $\mathrm{C}$ & $\mathrm{C}$ & $\mathrm{G}$ & $\mathrm{C}$ & $\bar{A}$ & $\bar{G}$ \\
\hline East-Asia & Japanese & JPN_J39 & $\bar{A}$ & $\mathrm{C}$ & $\mathrm{C}$ & $\mathrm{G}$ & $\mathrm{C}$ & $\bar{A}$ & $\overline{\mathrm{G}}$ \\
\hline East-Asia & Japanese & JPN_J40 & $\bar{A}$ & $\mathrm{C}$ & $\mathrm{C}$ & $\mathrm{G}$ & $\mathrm{C}$ & $\bar{A}$ & $\bar{G}$ \\
\hline East-Asia & Japanese & JPN_J40 & $\mathrm{A}$ & $\mathrm{C}$ & $\mathrm{C}$ & $\mathrm{G}$ & $\mathrm{C}$ & $\mathrm{A}$ & $\mathrm{G}$ \\
\hline East-Asia & Japanese & JPN_J41 & $\bar{A}$ & $\mathrm{C}$ & $\bar{C}$ & $\mathrm{G}$ & $\mathrm{C}$ & $\overline{\mathrm{A}}$ & $\bar{G}$ \\
\hline East-Asia & Japanese & JPN_J41 & $\bar{A}$ & $\mathrm{C}$ & $\mathrm{C}$ & $\mathrm{G}$ & $\mathrm{C}$ & $\mathrm{A}$ & $\mathrm{G}$ \\
\hline East-Asia & Japanese & JPN_J42 & $\overline{\mathrm{A}}$ & $\bar{C}$ & $\mathrm{C}$ & $\mathrm{G}$ & $\mathrm{C}$ & $\overline{\mathrm{A}}$ & $\bar{G}$ \\
\hline East-Asia & Japanese & JPN_J42 & A & $\mathrm{C}$ & $\mathrm{C}$ & $\mathrm{G}$ & $\mathrm{C}$ & $\bar{A}$ & $\mathrm{G}$ \\
\hline East-Asia & Japanese & JPN_JK1424 & $\mathrm{A}$ & $\mathrm{C}$ & $\mathrm{C}$ & $\mathrm{G}$ & $\mathrm{C}$ & $\bar{A}$ & $\mathrm{G}$ \\
\hline East-Asia & Japanese & JPN_JK1424 & $\bar{A}$ & $\mathrm{C}$ & $\mathrm{C}$ & $\mathrm{G}$ & $\mathrm{C}$ & $\bar{A}$ & $\bar{G}$ \\
\hline East-Asia & Japanese & JPN_JK1425 & $\bar{A}$ & $\mathrm{C}$ & $\mathrm{C}$ & $\mathrm{G}$ & $\mathrm{C}$ & $\mathrm{A}$ & $\bar{G}$ \\
\hline East-Asia & Japanese & JPN_JK1425 & $\overline{\mathrm{A}}$ & $\mathrm{C}$ & $\overline{\mathrm{C}}$ & $\mathrm{G}$ & $\bar{C}$ & $\overline{\mathrm{A}}$ & $\overline{\mathrm{G}}$ \\
\hline
\end{tabular}

\author{
John Wiley \& Sons, Inc.
}




\begin{tabular}{|c|c|c|c|c|c|c|c|c|c|}
\hline East-Asia & Japanese & JPN_JK1426 & $\mathrm{A}$ & $\mathrm{C}$ & $\mathrm{C}$ & $\mathrm{G}$ & $\mathrm{C}$ & $\overline{\mathrm{A}}$ & $\bar{G}$ \\
\hline East-Asia & Japanese & JPN_JK1426 & $\mathrm{A}$ & $\mathrm{C}$ & $\mathrm{C}$ & $\mathrm{G}$ & $\mathrm{C}$ & $\mathrm{A}$ & $\mathrm{G}$ \\
\hline East-Asia & Japanese & JPN_J30 & $\mathrm{A}$ & $\bar{C}$ & $\mathrm{C}$ & $\mathrm{G}$ & $\mathrm{C}$ & $\overline{\mathrm{A}}$ & $\bar{G}$ \\
\hline East-Asia & Japanese & JPN_J30 & $\mathrm{A}$ & $\mathrm{C}$ & $\mathrm{C}$ & $\mathrm{G}$ & $\mathrm{C}$ & $\mathrm{A}$ & $\mathrm{G}$ \\
\hline East-Asia & Japanese & JPN_JK1785 & $\mathrm{A}$ & $\bar{C}$ & $\bar{C}$ & $\mathrm{G}$ & $\mathrm{C}$ & $\bar{A}$ & $\mathrm{G}$ \\
\hline East-Asia & Japanese & JPN_JK1785 & $\mathrm{A}$ & $\mathrm{C}$ & $\mathrm{C}$ & $\mathrm{G}$ & $\mathrm{C}$ & $\mathrm{A}$ & $\bar{G}$ \\
\hline East-Asia & Japanese & JPN_JK1788 & $\mathrm{A}$ & $\mathrm{C}$ & $\mathrm{C}$ & $\mathrm{G}$ & $\mathrm{C}$ & $\mathrm{A}$ & $\bar{G}$ \\
\hline East-Asia & Japanese & JPN_JK1788 & $\mathrm{A}$ & $\bar{C}$ & $\mathrm{C}$ & $\mathrm{G}$ & $\mathrm{C}$ & $\overline{\mathrm{A}}$ & $\bar{G}$ \\
\hline East-Asia & Japanese & JPN_JK1790 & $\mathrm{A}$ & $\bar{C}$ & $\bar{C}$ & $\mathrm{G}$ & $\mathrm{C}$ & $\overline{\mathrm{A}}$ & $\bar{G}$ \\
\hline East-Asia & Japanese & JPN_JK1790 & $\mathrm{A}$ & $\bar{C}$ & $\bar{C}$ & $\mathrm{G}$ & $\mathrm{C}$ & $\overline{\mathrm{A}}$ & $\bar{G}$ \\
\hline East-Asia & Japanese & JPN_JK1793 & $\mathrm{A}$ & $\mathrm{C}$ & $\mathrm{C}$ & $\mathrm{G}$ & $\mathrm{C}$ & $\mathrm{A}$ & $\mathrm{G}$ \\
\hline East-Asia & Japanese & JPN_JK1793 & $\mathrm{A}$ & $C_{2}$ & $\mathrm{C}$ & $\mathrm{G}$ & $\mathrm{C}$ & $\overline{\mathrm{A}}$ & $\bar{G}$ \\
\hline East-Asia & Japanese & JPN_JK1850 & $\mathrm{A}$ & 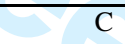 & $\mathrm{C}$ & $\mathrm{G}$ & $\mathrm{C}$ & A & $\mathrm{G}$ \\
\hline East-Asia & Japanese & JPN_JK1850 & $\mathrm{A}$ & $\bar{C}$ & $\mathrm{C}$ & $\mathrm{G}$ & $\mathrm{C}$ & $\bar{A}$ & $\bar{G}$ \\
\hline East-Asia & Japanese & JPN_JK1851 & $\mathrm{A}$ & $\mathrm{C}$ & $\mathrm{C}$ & $\mathrm{G}$ & $\mathrm{C}$ & A & $\mathrm{G}$ \\
\hline East-Asia & Japanese & JPN_JK1851 & A & $\mathrm{C}$ & $\mathrm{C}$ & $\mathrm{G}$ & $\mathrm{C}$ & $\bar{A}$ & $\bar{G}$ \\
\hline East-Asia & Japanese & JPN_JK1852 & $\mathrm{A}$ & $\bar{C}$ & $\mathrm{C}$ & $\mathrm{G}$ & $\mathrm{C}$ & $\mathrm{A}$ & $\bar{G}$ \\
\hline East-Asia & Japanese & JPN_JK1852 & $\mathrm{A}$ & $\bar{C}$ & $\bar{C}$ & $\mathrm{G}$ & $\mathrm{C}$ & $\overline{\mathrm{A}}$ & $\bar{G}$ \\
\hline East-Asia & Japanese & JPN_JK1853 & $\mathrm{A}$ & $\bar{C}$ & $\mathrm{C}$ & $\mathrm{G}$ & $\mathrm{C}$ & $\overline{\mathrm{A}}$ & $\bar{G}$ \\
\hline East-Asia & Japanese & JPN_JK1853 & $\mathrm{A}$ & $\mathrm{C}$ & $\mathrm{C}$ & $\mathrm{G}$ & $\mathrm{C}$ & A & $\mathrm{G}$ \\
\hline East-Asia & Japanese & JPN_JK1856 & $\mathrm{A}$ & $\bar{C}$ & $\mathrm{C}$ & $\mathrm{G}$ & $\mathrm{C}$ & $\overline{\mathrm{A}}$ & $\bar{G}$ \\
\hline East-Asia & Japanese & JPN_JK1856 & $\mathrm{A}$ & $\mathrm{C}$ & $\mathrm{C}$ & $\mathrm{G}$ & $\mathrm{C}$ & $\bar{A}$ & $\mathrm{G}$ \\
\hline East-Asia & Japanese & JPN_JK1857 & $\mathrm{A}$ & $\mathrm{C}$ & $\bar{C}$ & $\mathrm{G}$ & $\mathrm{C}$ & $\bar{A}$ & $\mathrm{G}$ \\
\hline East-Asia & Japanese & JPN_JK1857 & $\mathrm{A}$ & $\mathrm{C}$ & $\mathrm{C}$ & $\mathrm{G}$ & $\mathrm{C}$ & A & $\mathrm{G}$ \\
\hline East-Asia & Japanese & JPN_JK1860 & $\mathrm{A}$ & $\mathrm{C}$ & $\mathrm{C}$ & $\mathrm{G}$ & $\mathrm{C}$ & $\mathrm{A}$ & $\mathrm{G}$ \\
\hline East-Asia & Japanese & JPN_JK1860 & $\mathrm{A}$ & $\mathrm{C}$ & $\mathrm{C}$ & $\mathrm{G}$ & $\mathrm{C}$ & $\bar{A}$ & $\bar{G}$ \\
\hline East-Asia & Japanese & JPN_JK1863 & $\mathrm{A}$ & $\bar{C}$ & $\mathrm{C}$ & $\mathrm{G}$ & $\mathrm{C}$ & $\overline{\mathrm{A}}$ & $\bar{G}$ \\
\hline East-Asia & Japanese & JPN_JK1863 & $\mathrm{A}$ & $\bar{C}$ & $\mathrm{C}$ & $\mathrm{G}$ & $\mathrm{C}$ & $\overline{\mathrm{A}}$ & $\mathrm{G}$ \\
\hline
\end{tabular}

John Wiley 22 Sons, Inc. 


\begin{tabular}{|c|c|c|c|c|c|c|c|c|c|}
\hline East-Asia & Japanese & JPN_TMKH & $\bar{A}$ & $\mathrm{C}$ & $\bar{C}$ & $\mathrm{G}$ & $\mathrm{C}$ & $\mathrm{A}$ & $\overline{\mathrm{G}}$ \\
\hline East-Asia & Japanese & JPN_TMKH & $\overline{\mathrm{A}}$ & $\mathrm{C}$ & $\mathrm{C}$ & $\mathrm{G}$ & $\mathrm{C}$ & $\mathrm{A}$ & $\bar{G}$ \\
\hline Europe & Chuvash & CHU_JK4051 & $\overline{\mathrm{A}}$ & $\mathrm{C}$ & $\mathrm{C}$ & $\mathrm{G}$ & $\bar{C}$ & $\overline{\mathrm{A}}$ & $\bar{G}$ \\
\hline Europe & Chuvash & CHU_JK4051 & $\mathrm{A}$ & $\mathrm{C}$ & $\mathrm{C}$ & $\mathrm{G}$ & A & A & $\mathrm{G}$ \\
\hline Europe & Chuvash & CHU_JK4052 & $\overline{\mathrm{A}}$ & $\mathrm{C}$ & $\mathrm{C}$ & $\bar{G}$ & $\bar{C}$ & $\overline{\mathrm{A}}$ & $\bar{G}$ \\
\hline Europe & Chuvash & CHU_JK4052 & $\mathrm{A}$ & $\mathrm{C}$ & $\mathrm{C}$ & $\mathrm{G}$ & $\mathrm{C}$ & $\mathrm{A}$ & $\mathrm{G}$ \\
\hline Europe & Chuvash & CHU_JK4053 & $\mathrm{A}$ & $\mathrm{C}$ & $\mathrm{C}$ & $\mathrm{G}$ & $\mathrm{C}$ & $\mathrm{A}$ & $\mathrm{G}$ \\
\hline Europe & Chuvash & CHU_JK4053 & $\mathrm{A}$ & $\mathrm{C}$ & $\mathrm{C}$ & $\mathrm{G}$ & $\mathrm{C}$ & $\mathrm{A}$ & $\mathrm{G}$ \\
\hline Europe & Chuvash & CHU_JK4054 & $\overline{\mathrm{A}}$ & $\mathrm{C}$ & $\mathrm{C}$ & $\mathrm{G}$ & $\mathrm{C}$ & $\overline{\mathrm{A}}$ & $\bar{G}$ \\
\hline Europe & Chuvash & CHU_JK4054 & $\overline{\mathrm{A}}$ & $\mathrm{C}$ & $\mathrm{C}$ & $\mathrm{G}$ & $\mathrm{C}$ & $\overline{\mathrm{A}}$ & $\bar{G}$ \\
\hline Europe & Chuvash & CHU_JK4055 & $\overline{\mathrm{A}}$ & $\mathrm{C}$ & $\mathrm{C}$ & G & $\mathrm{C}$ & A & $\mathrm{G}$ \\
\hline Europe & Chuvash & CHU_JK4055 & $\bar{A}$ & $\mathrm{C}$ & $\bar{C}$ & $\mathrm{G}$ & $\mathrm{C}$ & $\mathrm{A}$ & $\overline{\mathrm{G}}$ \\
\hline Europe & Chuvash & CHU_JK4056 & $\mathrm{A}$ & $\mathrm{C}$ & $\mathrm{C}$ & $\mathrm{G}$ & $\mathrm{C}$ & $\mathrm{A}$ & $\bar{G}$ \\
\hline Europe & Chuvash & CHU_JK4056 & $\overline{\mathrm{A}}$ & $\mathrm{C}$ & $\mathrm{C}$ & $\bar{G}$ & $\mathrm{C}$ & $\bar{A}$ & $\overline{\mathrm{G}}$ \\
\hline Europe & Chuvash & CHU_JK4057 & $\mathrm{A}$ & $\mathrm{C}$ & $\mathrm{C}$ & $\mathrm{G}$ & $\mathrm{C}$ & $\mathrm{A}$ & $\mathrm{G}$ \\
\hline Europe & Chuvash & CHU_JK4057 & $\mathrm{A}$ & $\mathrm{C}$ & $\mathrm{C}$ & $\mathrm{G}$ & $\mathrm{C}$ & $\mathrm{A}$ & $\mathrm{G}$ \\
\hline Europe & Chuvash & CHU_JK4058 & $\mathrm{A}$ & $\mathrm{C}$ & $\mathrm{C}$ & $\mathrm{G}$ & $\mathrm{C}$ & $\bar{A}$ & $\bar{G}$ \\
\hline Europe & Chuvash & CHU_JK4058 & $\mathrm{A}$ & $\mathrm{C}$ & $\mathrm{C}$ & $\mathrm{G}$ & $\bar{C}$ & $\overline{\mathrm{A}}$ & $\mathrm{G}$ \\
\hline Europe & Chuvash & CHU_JK4059 & $\mathrm{A}$ & $\mathrm{C}$ & $\mathrm{C}$ & G & $\bar{C}$ & $\mathrm{~A}$ & $\bar{G}$ \\
\hline Europe & Chuvash & CHU_JK4059 & $\mathrm{A}$ & $\mathrm{C}$ & $\mathrm{C}$ & G & $\mathrm{C}$ & A & $\mathrm{A}$ \\
\hline Europe & Chuvash & CHU_JK4060 & $\mathrm{A}$ & $\mathrm{C}$ & $\mathrm{C}$ & $\bar{G}$ & $\mathrm{C}$ & $\bar{A}$ & $\bar{G}$ \\
\hline Europe & Chuvash & CHU_JK4060 & $\mathrm{A}$ & $\mathrm{C}$ & $\mathrm{C}$ & G & $\mathrm{C}$ & $\mathrm{A}$ & $\mathrm{G}$ \\
\hline Europe & Chuvash & CHU_JK4061 & $\mathrm{A}$ & $\mathrm{C}$ & $\mathrm{C}$ & $\mathrm{G}$ & $\mathrm{C}$ & $\mathrm{A}$ & $\mathrm{G}$ \\
\hline Europe & Chuvash & CHU_JK4061 & $\mathrm{A}$ & $\mathrm{C}$ & $\mathrm{C}$ & G & $\mathrm{C}$ & A & $\mathrm{G}$ \\
\hline Europe & Chuvash & CHU_JK4062 & A & $\mathrm{C}$ & $\mathrm{C}$ & $\mathrm{G}$ & $\mathrm{C}$ & A & $\mathrm{G}$ \\
\hline Europe & Chuvash & CHU_JK4062 & $\mathrm{A}$ & $\mathrm{C}$ & $\mathrm{C}$ & $\mathrm{G}$ & $\mathrm{C}$ & $\overline{\mathrm{A}}$ & $\bar{G}$ \\
\hline Europe & Chuvash & CHU_JK4063 & $\mathrm{A}$ & $\mathrm{C}$ & $\mathrm{C}$ & $\bar{G}$ & $\mathrm{C}$ & $\bar{A}$ & $\mathrm{G}$ \\
\hline Europe & Chuvash & CHU_JK4063 & $\mathrm{A}$ & $\bar{C}$ & $\mathrm{C}$ & $\bar{G}$ & $\mathrm{C}$ & $\bar{A}$ & $\mathrm{G}$ \\
\hline
\end{tabular}

John Wiley 23 Sons, Inc. 


\begin{tabular}{|c|c|c|c|c|c|c|c|c|c|}
\hline Europe & Chuvash & CHU_JK4064 & $\mathrm{A}$ & $\mathrm{C}$ & $\mathrm{C}$ & $\mathrm{G}$ & $\mathrm{C}$ & $\mathrm{A}$ & $\bar{G}$ \\
\hline Europe & Chuvash & CHU_JK4064 & $\mathrm{A}$ & $\mathrm{C}$ & $\mathrm{C}$ & $\mathrm{G}$ & $\mathrm{A}$ & $\mathrm{A}$ & $\mathrm{G}$ \\
\hline Europe & Chuvash & CHU_JK4065 & $\mathrm{A}$ & $\bar{C}$ & $\bar{C}$ & $\mathrm{G}$ & $\mathrm{C}$ & $\mathrm{A}$ & $\bar{G}$ \\
\hline Europe & Chuvash & CHU_JK4065 & $\mathrm{A}$ & $\mathrm{C}$ & $\mathrm{C}$ & $\mathrm{G}$ & $\mathrm{C}$ & $\mathrm{A}$ & $\mathrm{G}$ \\
\hline Europe & Chuvash & CHU_JK4066 & $\mathrm{A}$ & $\bar{C}$ & $\mathrm{C}$ & $\mathrm{G}$ & $\mathrm{C}$ & $\mathrm{A}$ & $\bar{G}$ \\
\hline Europe & Chuvash & CHU_JK4066 & $\mathrm{A}$ & $\mathrm{C}$ & $\mathrm{C}$ & $\mathrm{G}$ & $\mathrm{C}$ & $\mathrm{A}$ & $\bar{G}$ \\
\hline Europe & Chuvash & CHU_JK4067 & $\mathrm{A}$ & $\mathrm{C}$ & $\mathrm{C}$ & $\mathrm{G}$ & $\mathrm{C}$ & $\mathrm{A}$ & $\mathrm{G}$ \\
\hline Europe & Chuvash & CHU_JK4067 & $\mathrm{A}$ & $\mathrm{C}$ & $\bar{C}$ & $\mathrm{G}$ & $\mathrm{C}$ & $\bar{A}$ & $\mathrm{G}$ \\
\hline Europe & Chuvash & CHU_JK4068 & $\mathrm{A}$ & $\mathrm{C}$ & $\mathrm{C}$ & $\mathrm{G}$ & $\mathrm{C}$ & $\mathrm{A}$ & $\mathrm{G}$ \\
\hline Europe & Chuvash & CHU_JK4068 & $\mathrm{A}$ & $\mathrm{C}$ & $\bar{C}$ & $\mathrm{G}$ & $\mathrm{C}$ & $\bar{A}$ & $\mathrm{G}$ \\
\hline Europe & Chuvash & CHU_JK4069 & $\mathrm{A}$ & $\mathrm{C}$ & $\mathrm{C}$ & $\mathrm{G}$ & $\mathrm{C}$ & $\mathrm{A}$ & $\mathrm{G}$ \\
\hline Europe & Chuvash & CHU_JK4069 & $\mathrm{A}$ & 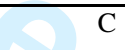 & $\mathrm{C}$ & $\mathrm{G}$ & $\mathrm{C}$ & $\mathrm{A}$ & $\bar{G}$ \\
\hline Europe & Chuvash & CHU_JK4070 & $\mathrm{A}$ & $\mathrm{C}$ & $\mathrm{C}$ & $\mathrm{G}$ & $\mathrm{C}$ & $\mathrm{A}$ & $\mathrm{G}$ \\
\hline Europe & Chuvash & CHU_JK4070 & $\mathrm{A}$ & $\mathrm{C}$ & $\mathrm{C}$ & $\mathrm{G}$ & $\mathrm{C}$ & $\mathrm{A}$ & $\bar{G}$ \\
\hline Europe & Chuvash & CHU_JK4071 & $\mathrm{A}$ & $\mathrm{C}$ & $\mathrm{C}$ & $\mathrm{G}$ & $\mathrm{C}$ & $\mathrm{A}$ & $\mathrm{G}$ \\
\hline Europe & Chuvash & CHU_JK4071 & $\mathrm{A}$ & $\mathrm{C}$ & $\mathrm{C}$ & $\mathrm{G}$ & $\mathrm{C}$ & $\mathrm{A}$ & $\mathrm{G}$ \\
\hline Europe & Chuvash & CHU_JK4072 & $\mathrm{A}$ & $\mathrm{C}$ & $\mathrm{C}$ & $\mathrm{G}$ & $\mathrm{C}$ & $\mathrm{A}$ & $\bar{G}$ \\
\hline Europe & Chuvash & CHU_JK4072 & $\mathrm{A}$ & $\mathrm{C}$ & $\mathrm{C}$ & $\mathrm{G}$ & $\mathrm{C}$ & $\mathrm{A}$ & $\bar{G}$ \\
\hline Europe & Chuvash & CHU_JK4073 & $\mathrm{A}$ & $\mathrm{C}$ & $\mathrm{C}$ & $\mathrm{G}$ & $\mathrm{C}$ & $\mathrm{A}$ & $\bar{G}$ \\
\hline Europe & Chuvash & CHU_JK4073 & $\mathrm{A}$ & $\mathrm{C}$ & $\mathrm{C}$ & $\mathrm{G}$ & $\mathrm{C}$ & $\mathrm{A}$ & $\mathrm{G}$ \\
\hline Europe & Chuvash & CHU_JK4074 & $\mathrm{A}$ & $\mathrm{C}$ & $\mathrm{C}$ & $\mathrm{G}$ & $\mathrm{C}$ & $\mathrm{A}$ & $\mathrm{G}$ \\
\hline Europe & Chuvash & CHU_JK4074 & $\mathrm{A}$ & $\mathrm{C}$ & $\mathrm{C}$ & $\mathrm{G}$ & $\mathrm{C}$ & $\mathrm{A}$ & $\mathrm{G}$ \\
\hline Europe & Danes & DAN_BK113 & $\mathrm{A}$ & $\mathrm{C}$ & $\mathrm{C}$ & $\mathrm{G}$ & $\mathrm{C}$ & $\mathrm{A}$ & $\bar{G}$ \\
\hline Europe & Danes & DAN_BK113 & $\mathrm{A}$ & $\mathrm{C}$ & $\mathrm{C}$ & $\mathrm{G}$ & $\mathrm{C}$ & $\mathrm{A}$ & $\mathrm{G}$ \\
\hline Europe & Danes & DAN_BK116 & $\mathrm{A}$ & $\mathrm{C}$ & $\mathrm{C}$ & $\mathrm{G}$ & $\mathrm{C}$ & $\mathrm{A}$ & $\mathrm{G}$ \\
\hline Europe & Danes & DAN_BK116 & $\mathrm{A}$ & $\mathrm{C}$ & $\bar{C}$ & $\mathrm{G}$ & $\mathrm{C}$ & $\bar{A}$ & $\mathrm{G}$ \\
\hline Europe & Danes & DAN_BK117 & $\mathrm{A}$ & $\mathrm{C}$ & $\mathrm{C}$ & $\mathrm{G}$ & $\mathrm{C}$ & $\mathrm{A}$ & $\bar{G}$ \\
\hline Europe & Danes & DAN_BK117 & $\mathrm{A}$ & $\mathrm{C}$ & $\mathrm{C}$ & $\mathrm{G}$ & $\mathrm{C}$ & $\mathrm{A}$ & $\bar{G}$ \\
\hline
\end{tabular}

John Wiley 24 Sons, Inc. 


\begin{tabular}{|c|c|c|c|c|c|c|c|c|c|}
\hline Europe & Danes & DAN_BK119 & $\bar{A}$ & $\mathrm{C}$ & $\bar{C}$ & $\bar{G}$ & $\mathrm{C}$ & $\bar{A}$ & $\mathrm{G}$ \\
\hline Europe & Danes & DAN_BK119 & $\mathrm{A}$ & $\mathrm{C}$ & $\mathrm{C}$ & $\mathrm{G}$ & $\mathrm{C}$ & $\bar{A}$ & $\mathrm{~A}$ \\
\hline Europe & Danes & DAN_BK120 & $\bar{A}$ & $\mathrm{C}$ & $\bar{C}$ & $\mathrm{G}$ & $\bar{C}$ & $\overline{\mathrm{A}}$ & $\bar{G}$ \\
\hline Europe & Danes & DAN_BK120 & $\bar{A}$ & $\mathrm{C}$ & $\mathrm{C}$ & $\mathrm{G}$ & $\mathrm{C}$ & $\mathrm{A}$ & $\mathrm{G}$ \\
\hline Europe & Danes & DAN_BK124 & $\overline{\mathrm{A}}$ & $\mathrm{C}$ & $\bar{C}$ & $\mathrm{G}$ & $\bar{C}$ & $\overline{\mathrm{A}}$ & $\bar{G}$ \\
\hline Europe & Danes & DAN_BK124 & $\mathrm{A}$ & $\mathrm{C}$ & C & $\mathrm{G}$ & $\mathrm{C}$ & A & $\mathrm{G}$ \\
\hline Europe & Danes & DAN_BK127 & $\mathrm{A}$ & $\mathrm{C}$ & C & $\mathrm{G}$ & $\mathrm{C}$ & A & $\mathrm{G}$ \\
\hline Europe & Danes & DAN_BK127 & A & $\mathrm{C}$ & C & $\mathrm{G}$ & $\mathrm{C}$ & A & $\mathrm{G}$ \\
\hline Europe & Danes & DAN_BK141 & $\overline{\mathrm{A}}$ & $\mathrm{C}$ & $\bar{C}$ & $\mathrm{G}$ & $\mathrm{C}$ & $\overline{\mathrm{A}}$ & $\bar{G}$ \\
\hline Europe & Danes & DAN_BK141 & $\overline{\mathrm{A}}$ & $\mathrm{C}$ & $\bar{C}$ & $\mathrm{G}$ & $\mathrm{C}$ & $\overline{\mathrm{A}}$ & $\bar{G}$ \\
\hline Europe & Danes & DAN_BK144 & G & $\mathrm{C}$ & $\bar{T}$ & $\mathrm{C}$ & $\mathrm{C}$ & A & G \\
\hline Europe & Danes & DAN_BK144 & $\bar{A}$ & $\mathrm{C}$ & $\bar{C}$ & $\mathrm{C}$ & $\mathrm{C}$ & $\overline{\mathrm{A}}$ & $\bar{G}$ \\
\hline Europe & Danes & DAN_BK146 & $\mathrm{A}$ & $\mathrm{T}$ & $\bar{C}$ & $\mathrm{C}$ & $\mathrm{C}$ & A & $\mathrm{G}$ \\
\hline Europe & Danes & DAN_BK146 & $\overline{\mathrm{A}}$ & $\mathrm{C}$ & $\bar{C}$ & $\mathrm{C}$ & $\bar{C}$ & $\overline{\mathrm{A}}$ & $\bar{G}$ \\
\hline Europe & Danes & DAN_BK153 & $\mathrm{A}$ & $\mathrm{C}$ & $\mathrm{C}$ & $\mathrm{C}$ & $\mathrm{C}$ & $\mathrm{A}$ & $\mathrm{G}$ \\
\hline Europe & Danes & DAN_BK153 & $\bar{A}$ & $\mathrm{C}$ & $\bar{C}$ & $\mathrm{C}$ & $\mathrm{C}$ & $\bar{A}$ & $\mathrm{G}$ \\
\hline Europe & Danes & DAN_BK157 & $\bar{A}$ & $\mathrm{C}$ & $\bar{C}$ & $\mathrm{C}$ & $\mathrm{C}$ & $\bar{A}$ & $\bar{G}$ \\
\hline Europe & Danes & DAN_BK157 & $\overline{\mathrm{A}}$ & $\bar{C}$ & $\bar{C}$ & $\mathrm{C}$ & $\bar{C}$ & $\overline{\mathrm{A}}$ & $\bar{G}$ \\
\hline Europe & Danes & DAN_BK160 & $\overline{\mathrm{A}}$ & $\bar{C}$ & $\bar{C}$ & $\mathrm{C}$ & $\bar{C}$ & $\overline{\mathrm{A}}$ & $\bar{G}$ \\
\hline Europe & Danes & DAN_BK160 & $\bar{A}$ & $\mathrm{C}$ & $\bar{C}$ & $\mathrm{C}$ & $\mathrm{C}$ & $\bar{A}$ & $\bar{G}$ \\
\hline Europe & Danes & DAN_BK171 & $\overline{\mathrm{A}}$ & $\mathrm{C}$ & $\bar{C}$ & $\mathrm{C}$ & $\mathrm{C}$ & $\overline{\mathrm{A}}$ & $\bar{G}$ \\
\hline Europe & Danes & DAN_BK171 & $\mathrm{A}$ & $\mathrm{C}$ & $\bar{C}$ & $\mathrm{C}$ & $\mathrm{C}$ & A & $\mathrm{G}$ \\
\hline Europe & Danes & DAN_BK172 & $\overline{\mathrm{A}}$ & $\bar{C}$ & $\bar{C}$ & $\mathrm{C}$ & $\bar{C}$ & $\overline{\mathrm{A}}$ & $\bar{G}$ \\
\hline Europe & Danes & DAN_BK172 & A & $\mathrm{C}$ & $\mathrm{C}$ & $\mathrm{C}$ & $\mathrm{C}$ & $\mathrm{A}$ & $\mathrm{G}$ \\
\hline Europe & Danes & DAN_BK180 & $\bar{A}$ & $\mathrm{C}$ & $\mathrm{C}$ & $\mathrm{C}$ & $\mathrm{C}$ & $\overline{\mathrm{A}}$ & G \\
\hline Europe & Danes & DAN_BK180 & $\overline{\mathrm{A}}$ & $\mathrm{C}$ & $\bar{C}$ & $\mathrm{C}$ & $\overline{\mathrm{A}}$ & $\overline{\mathrm{A}}$ & $\bar{G}$ \\
\hline Europe & Danes & DAN_JK3330 & $\mathrm{A}$ & $\mathrm{C}$ & $\bar{C}$ & $\mathrm{C}$ & $\mathrm{C}$ & $\mathrm{A}$ & $\mathrm{G}$ \\
\hline Europe & Danes & DAN_JK3330 & $\bar{A}$ & $\mathrm{C}$ & $\bar{C}$ & $\mathrm{c}$ & $\mathrm{C}$ & $\bar{A}$ & $\mathrm{G}$ \\
\hline
\end{tabular}

\author{
John Wiley ${ }^{25}$ Sons, Inc.
}




\begin{tabular}{|c|c|c|c|c|c|c|c|c|c|}
\hline Europe & Danes & DAN_JK3331 & $\overline{\mathrm{A}}$ & $\mathrm{C}$ & $\mathrm{C}$ & $\bar{G}$ & $\mathrm{C}$ & $\overline{\mathrm{A}}$ & $\bar{G}$ \\
\hline Europe & Danes & DAN_JK3331 & $\bar{A}$ & $\mathrm{C}$ & $\mathrm{C}$ & G & $\mathrm{C}$ & $\bar{A}$ & $\mathrm{G}$ \\
\hline Europe & Danes & DAN_JK3332 & $\overline{\mathrm{A}}$ & $\bar{C}$ & $\mathrm{C}$ & $\bar{G}$ & $\bar{C}$ & $\overline{\mathrm{A}}$ & $\bar{G}$ \\
\hline Europe & Danes & DAN_JK3332 & $\mathrm{A}$ & $\mathrm{C}$ & $\mathrm{C}$ & G & $\mathrm{A}$ & $\mathrm{A}$ & $\mathrm{G}$ \\
\hline Europe & Danes & DAN_JK3333 & $\overline{\mathrm{A}}$ & $\bar{C}$ & $\mathrm{C}$ & $\bar{G}$ & $\bar{C}$ & $\overline{\mathrm{A}}$ & $\bar{G}$ \\
\hline Europe & Danes & DAN_JK3333 & $\bar{A}$ & $\mathrm{C}$ & $\mathrm{C}$ & G & $\mathrm{C}$ & $\bar{A}$ & $\mathrm{G}$ \\
\hline Europe & Danes & DAN_JK3334 & $\mathrm{A}$ & $\mathrm{C}$ & $\mathrm{C}$ & G & $\mathrm{C}$ & $\mathrm{A}$ & $\mathrm{G}$ \\
\hline Europe & Danes & DAN_JK3334 & $\mathrm{A}$ & $\bar{C}$ & $\mathrm{C}$ & $\bar{G}$ & $\mathrm{C}$ & $\bar{A}$ & $\mathrm{G}$ \\
\hline Europe & Danes & DAN_JK3335 & $\mathrm{A}$ & $\mathrm{C}$ & $\mathrm{C}$ & $\mathrm{G}$ & $\mathrm{C}$ & $\bar{A}$ & $\mathrm{G}$ \\
\hline Europe & Danes & DAN_JK3335 & $\mathrm{A}$ & $\mathrm{C}$ & $\mathrm{C}$ & $\mathrm{G}$ & $\mathrm{C}$ & $\mathrm{A}$ & $\mathrm{G}$ \\
\hline Europe & Danes & DAN_JK3336 & A & $\mathrm{C}$ & $\mathrm{C}$ & $\mathrm{G}$ & $\mathrm{C}$ & A & $\mathrm{G}$ \\
\hline Europe & Danes & DAN_JK3336 & $\mathrm{A}$ & $\mathrm{C}$ & $\mathrm{C}$ & $\mathrm{G}$ & $\bar{A}$ & $\bar{A}$ & $\bar{G}$ \\
\hline
\end{tabular}


Supp. Table S3. Genotype information per individual of the TLR6 non-synonymous variants

\begin{tabular}{|c|c|c|c|c|c|c|c|c|c|c|c|c|c|c|c|c|c|c|c|}
\hline & & & SNP & SNP & SNP & SNP & SNP & SNP & SNP & SNP & SNP & SNP & SNP & SNP & SNP & SNP & SNP & SNP & SNP \\
\hline \begin{tabular}{|c|}
$\begin{array}{c}\text { Contin } \\
\text { ent }\end{array}$ \\
\end{tabular} & $\begin{array}{l}\text { Populat } \\
\text { ion }\end{array}$ & ID & $\begin{array}{l}\text { c.359T } \\
>C\end{array}$ & $\begin{array}{l}\text { c.382C } \\
>G\end{array}$ & $\begin{array}{l}\text { c.581T } \\
>C\end{array}$ & $\begin{array}{l}\text { c.628G } \\
>A\end{array}$ & $\begin{array}{l}c .629 C \\
>G\end{array}$ & $\begin{array}{l}\text { c.740G } \\
>A\end{array}$ & \begin{tabular}{|l|}
$\begin{array}{l}\text { c.745C } \\
>\mathrm{T}\end{array}$ \\
\end{tabular} & $\begin{array}{l}\text { c.847A } \\
>G\end{array}$ & $\begin{array}{l}\text { c.979G } \\
>A\end{array}$ & $\begin{array}{l}\text { c. } 1280 \\
T>C\end{array}$ & $\begin{array}{l}\text { C. } 1325 \\
A>C\end{array}$ & $\begin{array}{l}\text { c.1393 } \\
\mathrm{G}>\mathrm{A}\end{array}$ & $\begin{array}{l}\text { c. } 1420 \\
G>A\end{array}$ & $\begin{array}{l}c .1421 \\
C>T\end{array}$ & $\begin{array}{l}c .1775 T \\
>G\end{array}$ & $\begin{array}{l}\text { c. } 2069 \\
A>C\end{array}$ & $\begin{array}{l}\text { c. } 2124 \\
G>C\end{array}$ \\
\hline $\begin{array}{c}\text { AFRIC } \\
\text { A }\end{array}$ & $\begin{array}{c}\text { Chagg } \\
\text { a }\end{array}$ & $\underset{4125}{\text { CHA_JK }}$ & $\mathrm{T}$ & $\mathrm{C}$ & $\mathrm{T}$ & $\mathrm{G}$ & $\mathrm{C}$ & $\mathrm{G}$ & $\mathrm{C}$ & $\mathrm{A}$ & $\mathrm{G}$ & $\mathrm{T}$ & $\mathrm{A}$ & G & $\mathrm{G}$ & $\mathrm{C}$ & $\mathrm{G}$ & $\mathrm{A}$ & $\mathrm{G}$ \\
\hline $\begin{array}{c}\text { AFRIC } \\
\text { A }\end{array}$ & $\begin{array}{c}\text { Chagg } \\
\text { a }\end{array}$ & $\begin{array}{c}\text { CHA_JK } \\
4125\end{array}$ & $\bar{C}$ & $\mathrm{C}$ & $\mathrm{T}$ & $\mathrm{G}$ & $\mathrm{C}$ & $\mathrm{G}$ & $\mathrm{C}$ & $\mathrm{A}$ & $\mathrm{G}$ & $\mathrm{T}$ & $\mathrm{A}$ & $\bar{A}$ & $\bar{G}$ & $\mathrm{C}$ & $\bar{G}$ & $\mathrm{~A}$ & $\bar{G}$ \\
\hline $\begin{array}{c}\text { AFRIC } \\
\text { A }\end{array}$ & $\begin{array}{c}\text { Chagg } \\
\text { a }\end{array}$ & $\begin{array}{c}\text { CHA_JK } \\
4126 \\
\end{array}$ & $\mathrm{~T}$ & $\bar{C}$ & $\mathrm{~T}$ & $\bar{G}$ & $\bar{C}$ & $\overline{\mathrm{A}}$ & $\mathrm{C}$ & $\overline{\mathrm{A}}$ & $\bar{G}$ & $\mathrm{~T}$ & $\overline{\mathrm{A}}$ & $\bar{G}$ & $\bar{G}$ & $\bar{C}$ & $\bar{G}$ & $\overline{\mathrm{A}}$ & $\bar{G}$ \\
\hline $\begin{array}{c}\text { AFRIC } \\
\text { A }\end{array}$ & $\begin{array}{c}\text { Chagg } \\
a\end{array}$ & $\underset{4126}{\text { CHA_JK }}$ & $\mathrm{T}$ & $\mathrm{C}$ & $\mathrm{T}$ & $\mathrm{G}$ & $\mathrm{C}$ & $\mathrm{G}$ & $\mathrm{C}$ & $\bar{A}$ & $\mathrm{G}$ & $\mathrm{T}$ & $\mathrm{A}$ & $\mathrm{G}$ & $\mathrm{G}$ & $\mathrm{C}$ & $\mathrm{G}$ & $\mathrm{A}$ & $\bar{G}$ \\
\hline $\begin{array}{c}\text { AFRIC } \\
\text { A }\end{array}$ & $\begin{array}{c}\text { Chagg } \\
\text { a }\end{array}$ & $\underset{4127}{\text { CHA_JK }}$ & $\mathrm{T}$ & $\mathrm{C}$ & $\mathrm{T}$ & $\bar{G}$ & $\mathrm{C}$ & $\bar{G}$ & $\mathrm{C}$ & $\mathrm{A}$ & $\bar{G}$ & $\mathrm{~T}$ & $\mathrm{~A}$ & $\bar{G}$ & $\mathrm{G}$ & $\mathrm{C}$ & $\mathrm{G}$ & $\overline{\mathrm{A}}$ & $\bar{G}$ \\
\hline $\begin{array}{c}\text { AFRIC } \\
\text { A }\end{array}$ & $\begin{array}{c}\text { Chagg } \\
\text { a }\end{array}$ & $\begin{array}{c}\text { CHA_JK } \\
4127\end{array}$ & $\mathrm{~T}$ & $\mathrm{C}$ & $\mathrm{T}$ & $\mathrm{G}$ & $\bar{C}$ & $\overline{\mathrm{A}}$ & $\mathrm{C}$ & $\overline{\mathrm{A}}$ & $\bar{G}$ & $\mathrm{~T}$ & $\overline{\mathrm{A}}$ & $\mathrm{G}$ & $\bar{G}$ & $\bar{C}$ & $\bar{G}$ & $\overline{\mathrm{A}}$ & $\bar{G}$ \\
\hline $\begin{array}{c}\text { AFRIC } \\
\text { A }\end{array}$ & $\begin{array}{c}\text { Chagg } \\
\text { a }\end{array}$ & $\begin{array}{c}\text { CHA_JK } \\
4128 \\
\end{array}$ & $\mathrm{~T}$ & $\mathrm{C}$ & $\mathrm{T}$ & $\mathrm{G}$ & $\mathrm{C}$ & $\mathrm{G}$ & $\mathrm{C}$ & $\mathrm{A}$ & $\mathrm{G}$ & $\mathrm{T}$ & $\mathrm{A}$ & $\mathrm{G}$ & $\mathrm{A}$ & $\mathrm{C}$ & $\mathrm{G}$ & $\mathrm{A}$ & G \\
\hline $\begin{array}{c}\text { AFRIC } \\
\text { A }\end{array}$ & $\begin{array}{c}\text { Chagg } \\
\text { a }\end{array}$ & $\underset{4128}{\text { CHA_JK }}$ & $\mathrm{T}$ & $\mathrm{C}$ & $\mathrm{T}$ & $\bar{G}$ & $\mathrm{C}$ & $\bar{G}$ & $\mathrm{~T}$ & $\bar{A}$ & $\bar{G}$ & $\mathrm{~T}$ & $\overline{\mathrm{A}}$ & $\bar{G}$ & $\bar{G}$ & $\mathrm{C}$ & $\mathrm{G}$ & $\overline{\mathrm{A}}$ & $\bar{G}$ \\
\hline $\begin{array}{c}\text { AFRIC } \\
\text { A }\end{array}$ & $\begin{array}{c}\text { Chagg } \\
\text { a }\end{array}$ & $\begin{array}{c}\text { CHA_JK } \\
4129\end{array}$ & $\mathrm{~T}$ & $\mathrm{C}$ & $\mathrm{T}$ & G & $\mathrm{C}$ & G & $\mathrm{C}$ & A & G & $\mathrm{T}$ & $\mathrm{A}$ & G & G & $\mathrm{C}$ & G & $\mathrm{A}$ & $\mathrm{C}$ \\
\hline $\begin{array}{c}\text { AFRIC } \\
\text { A }\end{array}$ & $\begin{array}{c}\text { Chagg } \\
\text { a }\end{array}$ & $\begin{array}{c}\text { CHA_JK } \\
4129 \\
\end{array}$ & $\mathrm{~T}$ & $\mathrm{C}$ & $\mathrm{T}$ & G & $\mathrm{C}$ & G & $\mathrm{C}$ & $\bar{A}$ & $\mathrm{G}$ & $\mathrm{T}$ & $\mathrm{A}$ & G & G & $\mathrm{C}$ & G & $\mathrm{A}$ & G \\
\hline $\begin{array}{c}\text { AFRIC } \\
\text { A }\end{array}$ & $\begin{array}{c}\text { Chagg } \\
a\end{array}$ & $\begin{array}{c}\text { CHA_JK } \\
4130\end{array}$ & $\mathrm{~T}$ & $\mathrm{C}$ & $\mathrm{T}$ & G & $\mathrm{C}$ & G & $\mathrm{C}$ & $\mathrm{A}$ & G & $\mathrm{T}$ & $\mathrm{A}$ & G & G & $\mathrm{C}$ & G & $\mathrm{A}$ & G \\
\hline $\begin{array}{c}\text { AFRIC } \\
\text { A }\end{array}$ & $\begin{array}{c}\text { Chagg } \\
\text { a }\end{array}$ & $\begin{array}{c}\text { CHA_JK } \\
4130\end{array}$ & $\bar{T}$ & $\mathrm{C}$ & $\mathrm{T}$ & $\mathrm{G}$ & $\mathrm{C}$ & $\bar{G}$ & $\mathrm{C}$ & $\bar{A}$ & $\bar{G}$ & $\mathrm{~T}$ & $\bar{A}$ & $\mathrm{G}$ & $\mathrm{G}$ & $\mathrm{C}$ & $\mathrm{G}$ & $\mathrm{C}$ & $\mathrm{G}$ \\
\hline $\begin{array}{c}\text { AFRIC } \\
\text { A }\end{array}$ & $\begin{array}{c}\text { Chagg } \\
\text { a }\end{array}$ & $\begin{array}{c}\text { CHA_JK } \\
4131 \\
\end{array}$ & $\mathrm{~T}$ & $\mathrm{C}$ & $\mathrm{T}$ & $\mathrm{G}$ & $\mathrm{C}$ & $\mathrm{G}$ & $\mathrm{C}$ & $\mathrm{A}$ & $\mathrm{G}$ & $\mathrm{T}$ & $\mathrm{A}$ & $\mathrm{G}$ & $\mathrm{G}$ & $\mathrm{C}$ & $\mathrm{G}$ & $\bar{A}$ & $\mathrm{G}$ \\
\hline $\begin{array}{c}\text { AFRIC } \\
\text { A }\end{array}$ & $\begin{array}{c}\text { Chagg } \\
a\end{array}$ & $\underset{4131}{\text { CHA_JK }}$ & $\mathrm{T}$ & $\mathrm{C}$ & $\mathrm{C}$ & $\mathrm{G}$ & $\mathrm{C}$ & $\mathrm{G}$ & $\mathrm{C}$ & $\bar{A}$ & $\mathrm{G}$ & $\mathrm{T}$ & $\mathrm{A}$ & $\bar{G}$ & $\bar{G}$ & $\mathrm{C}$ & $\bar{G}$ & $\overline{\mathrm{A}}$ & $\bar{G}$ \\
\hline $\begin{array}{c}\text { AFRIC } \\
\text { A }\end{array}$ & $\begin{array}{c}\text { Chagg } \\
\text { a }\end{array}$ & $\begin{array}{c}\text { CHA_JK } \\
4132\end{array}$ & $\mathrm{~T}$ & $\mathrm{C}$ & $\mathrm{T}$ & $\mathrm{G}$ & $\mathrm{C}$ & $\mathrm{G}$ & $\mathrm{C}$ & $\mathrm{A}$ & $\mathrm{G}$ & $\mathrm{T}$ & $\mathrm{A}$ & $\mathrm{G}$ & $\mathrm{G}$ & $\mathrm{C}$ & $\mathrm{G}$ & $\mathrm{A}$ & $\mathrm{G}$ \\
\hline $\begin{array}{c}\text { AFRIC } \\
\text { A }\end{array}$ & $\begin{array}{c}\text { Chagg } \\
\text { a }\end{array}$ & $\begin{array}{c}\text { CHA_JK } \\
4132 \\
\end{array}$ & $\mathrm{~T}$ & $\mathrm{C}$ & $\mathrm{T}$ & $\mathrm{G}$ & $\mathrm{C}$ & $\bar{G}$ & $\mathrm{~T}$ & $\mathrm{G}$ & $\bar{G}$ & $\mathrm{~T}$ & $\overline{\mathrm{A}}$ & $\mathrm{G}$ & $\mathrm{G}$ & $\mathrm{C}$ & $\bar{G}$ & $\overline{\mathrm{A}}$ & $\bar{G}$ \\
\hline $\begin{array}{c}\text { AFRIC } \\
\text { A }\end{array}$ & $\begin{array}{c}\text { Chagg } \\
a\end{array}$ & $\underset{4133}{\text { CHA_JK }}$ & $\mathrm{C}$ & $\mathrm{C}$ & $\mathrm{T}$ & $\mathrm{G}$ & $\mathrm{C}$ & $\mathrm{G}$ & $\mathrm{C}$ & $\mathrm{A}$ & $\mathrm{G}$ & $\mathrm{T}$ & $\mathrm{A}$ & $\mathrm{A}$ & $\mathrm{G}$ & $\mathrm{C}$ & $\mathrm{G}$ & $\mathrm{A}$ & $\mathrm{G}$ \\
\hline $\begin{array}{c}\text { AFRIC } \\
\text { A }\end{array}$ & $\begin{array}{c}\text { Chagg } \\
a\end{array}$ & $\underset{4133}{\text { CHA_JK }}$ & $\mathrm{T}$ & $\mathrm{C}$ & $\mathrm{T}$ & $\mathrm{G}$ & $\mathrm{C}$ & $\bar{G}$ & $\mathrm{C}$ & $\overline{\mathrm{A}}$ & $\bar{G}$ & $\mathrm{~T}$ & $\mathrm{~A}$ & $\mathrm{G}$ & $\mathrm{G}$ & $\mathrm{C}$ & $\mathrm{G}$ & $\bar{A}$ & $\mathrm{G}$ \\
\hline $\begin{array}{c}\text { AFRIC } \\
\text { A }\end{array}$ & $\begin{array}{c}\text { Chagg } \\
\text { a }\end{array}$ & $\begin{array}{c}\text { CHA_JK } \\
4134\end{array}$ & $\mathrm{~T}$ & $\mathrm{C}$ & $\mathrm{T}$ & $\mathrm{G}$ & $\mathrm{C}$ & $\mathrm{G}$ & $\mathrm{C}$ & $\mathrm{A}$ & $\mathrm{G}$ & $\mathrm{T}$ & $\mathrm{A}$ & $\mathrm{G}$ & $\mathrm{G}$ & $\mathrm{C}$ & $\mathrm{G}$ & $\mathrm{A}$ & $\mathrm{G}$ \\
\hline $\begin{array}{c}\text { AFRIC } \\
\text { A }\end{array}$ & $\begin{array}{c}\text { Chagg } \\
a\end{array}$ & $\underset{4134}{\text { CHA_JK }}$ & $\mathrm{T}$ & $\mathrm{C}$ & $\mathrm{T}$ & $\mathrm{G}$ & $\mathrm{C}$ & $\mathrm{G}$ & $\mathrm{C}$ & $\bar{A}$ & $\mathrm{G}$ & $\mathrm{T}$ & $\mathrm{A}$ & $\mathrm{G}$ & $\mathrm{G}$ & $\mathrm{C}$ & $\mathrm{G}$ & $\bar{A}$ & $\mathrm{G}$ \\
\hline
\end{tabular}

John Wiley 27 Sons, Inc. 


\begin{tabular}{|c|c|c|c|c|c|c|c|c|c|c|c|c|c|c|c|c|c|c|c|}
\hline $\begin{array}{c}\text { AFRIC } \\
\text { A }\end{array}$ & $\begin{array}{c}\text { Chagg } \\
\text { a }\end{array}$ & $\begin{array}{c}\text { CHA_JK } \\
4135\end{array}$ & $\mathrm{~T}$ & $\bar{C}$ & $\mathrm{~T}$ & $\mathrm{G}$ & $\mathrm{C}$ & $\mathrm{G}$ & $\mathrm{C}$ & $\bar{A}$ & $\mathrm{G}$ & $\mathrm{T}$ & $\mathrm{A}$ & $\bar{G}$ & $\mathrm{G}$ & $\mathrm{C}$ & $\mathrm{G}$ & $\mathrm{A}$ & $\bar{G}$ \\
\hline $\begin{array}{c}\text { AFRIC } \\
\text { A }\end{array}$ & $\begin{array}{c}\text { Chagg } \\
\text { a }\end{array}$ & $\begin{array}{c}\text { CHA_JK } \\
4135\end{array}$ & $\mathrm{~T}$ & $\mathrm{C}$ & $\mathrm{T}$ & $\mathrm{G}$ & $\mathrm{C}$ & $\mathrm{G}$ & $\mathrm{C}$ & $\mathrm{A}$ & $\mathrm{G}$ & $\mathrm{T}$ & $\mathrm{A}$ & $\mathrm{G}$ & $\mathrm{G}$ & $\mathrm{C}$ & $\mathrm{G}$ & $\mathrm{A}$ & $\mathrm{G}$ \\
\hline $\begin{array}{c}\text { AFRIC } \\
\text { A }\end{array}$ & $\begin{array}{c}\text { Chagg } \\
a\end{array}$ & $\begin{array}{c}\text { CHA_JK } \\
4136 \\
\end{array}$ & $\mathrm{C}$ & $\mathrm{C}$ & $\mathrm{T}$ & $\mathrm{G}$ & $\bar{C}$ & $\mathrm{G}$ & $\mathrm{C}$ & $\mathrm{A}$ & $\mathrm{G}$ & $\mathrm{T}$ & $\mathrm{A}$ & $\mathrm{A}$ & $\mathrm{G}$ & $\mathrm{C}$ & $\mathrm{G}$ & $\mathrm{A}$ & $\mathrm{G}$ \\
\hline $\begin{array}{c}\text { AFRIC } \\
\text { A }\end{array}$ & $\begin{array}{c}\text { Chagg } \\
\text { a }\end{array}$ & $\begin{array}{c}\text { CHA_JK } \\
4136\end{array}$ & $\mathrm{~T}$ & $\mathrm{C}$ & $\mathrm{C}$ & $\mathrm{G}$ & $\mathrm{C}$ & $\mathrm{G}$ & $\mathrm{C}$ & $\mathrm{A}$ & $\mathrm{G}$ & $\mathrm{T}$ & $\mathrm{A}$ & $\mathrm{G}$ & $\mathrm{G}$ & C & $\mathrm{G}$ & $\mathrm{A}$ & $\mathrm{G}$ \\
\hline $\begin{array}{c}\text { AFRIC } \\
\text { A }\end{array}$ & $\begin{array}{c}\text { Chagg } \\
a\end{array}$ & $\begin{array}{c}\text { CHA_JK } \\
4137\end{array}$ & $\mathrm{~T}$ & $\mathrm{C}$ & $\mathrm{C}$ & $\mathrm{G}$ & $\mathrm{C}$ & $\mathrm{G}$ & $\mathrm{C}$ & $\mathrm{A}$ & $\mathrm{G}$ & $\mathrm{T}$ & $\mathrm{A}$ & $\mathrm{G}$ & $\mathrm{G}$ & $\mathrm{C}$ & $\mathrm{G}$ & $\mathrm{A}$ & $\mathrm{G}$ \\
\hline $\begin{array}{c}\text { AFRIC } \\
\text { A }\end{array}$ & $\begin{array}{c}\text { Chagg } \\
\text { a }\end{array}$ & $\begin{array}{c}\text { CHA_JK } \\
4137\end{array}$ & $\mathrm{~T}$ & $\mathrm{C}$ & $\mathrm{T}$ & $\mathrm{G}$ & $\mathrm{C}$ & $\mathrm{G}$ & $\mathrm{C}$ & A & $\mathrm{G}$ & $\mathrm{T}$ & $\mathrm{A}$ & $\mathrm{G}$ & $\mathrm{G}$ & $\mathrm{C}$ & $\mathrm{G}$ & $\mathrm{A}$ & $\mathrm{G}$ \\
\hline $\begin{array}{c}\text { AFRIC } \\
\text { A }\end{array}$ & $\begin{array}{c}\text { Chagg } \\
\text { a }\end{array}$ & $\begin{array}{c}\text { CHA_JK } \\
4138\end{array}$ & $\mathrm{C}$ & $\mathrm{C}$ & $\mathrm{T}$ & $\mathrm{G}$ & $\mathrm{C}$ & $\mathrm{G}$ & $\mathrm{C}$ & $\mathrm{A}$ & $\mathrm{G}$ & $\mathrm{T}$ & $\mathrm{A}$ & $\mathrm{G}$ & $\mathrm{G}$ & $\mathrm{C}$ & $\mathrm{G}$ & $\mathrm{A}$ & $\bar{G}$ \\
\hline $\begin{array}{c}\text { AFRIC } \\
\text { A }\end{array}$ & $\begin{array}{c}\text { Chagg } \\
a\end{array}$ & $\begin{array}{c}\text { CHA_JK } \\
4138\end{array}$ & $\mathrm{~T}$ & $\mathrm{C}$ & $\mathrm{T}$ & $\mathrm{G}$ & $\mathrm{C}$ & $\mathrm{G}$ & $\mathrm{C}$ & $\mathrm{A}$ & $\mathrm{G}$ & $\mathrm{T}$ & $\mathrm{A}$ & $\mathrm{G}$ & $\mathrm{G}$ & $\mathrm{C}$ & $\mathrm{G}$ & A & $\bar{G}$ \\
\hline $\begin{array}{c}\text { AFRIC } \\
\text { A }\end{array}$ & $\begin{array}{c}\text { Chagg } \\
\text { a }\end{array}$ & $\begin{array}{c}\text { CHA_JK } \\
4139\end{array}$ & $\mathrm{~T}$ & $\mathrm{C}$ & $\mathrm{T}$ & $\mathrm{G}$ & $\mathrm{C}$ & $\mathrm{G}$ & $\mathrm{C}$ & A & $\mathrm{G}$ & $\mathrm{T}$ & $\mathrm{A}$ & $\mathrm{G}$ & $\mathrm{G}$ & $\mathrm{C}$ & $\mathrm{G}$ & $\mathrm{A}$ & $\mathrm{C}$ \\
\hline $\begin{array}{c}\text { AFRIC } \\
\text { A }\end{array}$ & $\begin{array}{c}\text { Chagg } \\
\text { a }\end{array}$ & $\begin{array}{c}\text { CHA_JK } \\
4139\end{array}$ & $\mathrm{~T}$ & $\mathrm{C}$ & $\mathrm{T}$ & $\mathrm{G}$ & $\mathrm{C}$ & $\mathrm{G}$ & $\mathrm{C}$ & $\mathrm{A}$ & $\mathrm{G}$ & $\mathrm{T}$ & $\mathrm{A}$ & $\mathrm{G}$ & $\mathrm{G}$ & C & $\mathrm{G}$ & $\mathrm{A}$ & $\bar{G}$ \\
\hline $\begin{array}{c}\text { AFRIC } \\
\text { A }\end{array}$ & $\begin{array}{c}\text { Chagg } \\
a\end{array}$ & $\begin{array}{c}\text { CHA_JK } \\
4140\end{array}$ & $\mathrm{~T}$ & $\mathrm{C}$ & $\mathrm{T}$ & $\mathrm{G}$ & $\mathrm{C}$ & $\mathrm{G}$ & $\mathrm{C}$ & $\mathrm{A}$ & $\mathrm{G}$ & $\mathrm{T}$ & $\mathrm{A}$ & $\mathrm{G}$ & $\mathrm{G}$ & $\mathrm{C}$ & $\mathrm{G}$ & $\mathrm{A}$ & $\overline{\mathrm{G}}$ \\
\hline $\begin{array}{c}\text { AFRIC } \\
\text { A }\end{array}$ & $\begin{array}{c}\text { Chagg } \\
a\end{array}$ & $\begin{array}{c}\text { CHA_JK } \\
4140 \\
\end{array}$ & $\mathrm{~T}$ & $\mathrm{C}$ & $\mathrm{T}$ & $\mathrm{G}$ & $\mathrm{C}$ & $\mathrm{G}$ & $\mathrm{C}$ & A & $\mathrm{G}$ & $\mathrm{T}$ & A & $\mathrm{G}$ & $\mathrm{G}$ & $\mathrm{C}$ & $\mathrm{G}$ & $\mathrm{A}$ & G \\
\hline $\begin{array}{c}\text { AFRIC } \\
\text { A }\end{array}$ & $\begin{array}{c}\text { Chagg } \\
\text { a }\end{array}$ & $\begin{array}{c}\text { CHA_JK } \\
4141\end{array}$ & $\mathrm{~T}$ & $\mathrm{C}$ & $\mathrm{T}$ & $\mathrm{G}$ & $\mathrm{C}$ & $\mathrm{G}$ & $\mathrm{C}$ & $\mathrm{A}$ & $\mathrm{G}$ & $\mathrm{T}$ & $\mathrm{A}$ & $\mathrm{G}$ & $\mathrm{G}$ & $\mathrm{C}$ & $\mathrm{G}$ & $\mathrm{A}$ & $\bar{G}$ \\
\hline $\begin{array}{c}\text { AFRIC } \\
\text { A }\end{array}$ & $\begin{array}{c}\text { Chagg } \\
a\end{array}$ & $\begin{array}{c}\text { CHA_JK } \\
4141\end{array}$ & $\mathrm{~T}$ & $\bar{C}$ & $\mathrm{~T}$ & $\mathrm{G}$ & $\bar{C}$ & $\mathrm{G}$ & $\mathrm{C}$ & $\bar{A}$ & $\mathrm{G}$ & $\mathrm{T}$ & $\mathrm{A}$ & $\bar{G}$ & $\mathrm{G}$ & $\mathrm{C}$ & $\mathrm{G}$ & $\bar{A}$ & $\overline{\mathrm{G}}$ \\
\hline $\begin{array}{c}\text { AFRIC } \\
\text { A }\end{array}$ & $\begin{array}{c}\text { Chagg } \\
\text { a } \\
\end{array}$ & $\begin{array}{c}\text { CHA_JK } \\
4142 \\
\end{array}$ & $\mathrm{~T}$ & $\mathrm{C}$ & $\mathrm{T}$ & $\mathrm{G}$ & $\bar{C}$ & $\mathrm{G}$ & $\mathrm{C}$ & $\mathrm{A}$ & $\mathrm{G}$ & $\mathrm{T}$ & $\mathrm{A}$ & $\mathrm{G}$ & $\mathrm{G}$ & $\bar{C}$ & $\mathrm{G}$ & $\mathrm{A}$ & $\bar{G}$ \\
\hline $\begin{array}{c}\text { AFRIC } \\
\text { A }\end{array}$ & $\begin{array}{c}\text { Chagg } \\
\text { a }\end{array}$ & $\begin{array}{c}\text { CHA_JK } \\
4142 \\
\end{array}$ & $\bar{T}$ & $\mathrm{C}$ & $\mathrm{T}$ & $\mathrm{G}$ & $\bar{C}$ & $\mathrm{G}$ & $\mathrm{C}$ & A & $\mathrm{G}$ & $\mathrm{T}$ & $\mathrm{A}$ & $\mathrm{G}$ & $\mathrm{G}$ & $\mathrm{C}$ & $\mathrm{G}$ & $\mathrm{A}$ & $\mathrm{G}$ \\
\hline $\begin{array}{c}\text { AFRIC } \\
\text { A }\end{array}$ & $\begin{array}{c}\text { Chagg } \\
a\end{array}$ & $\begin{array}{c}\text { CHA_JK } \\
4143 \\
\end{array}$ & $\mathrm{~T}$ & $\mathrm{C}$ & $\mathrm{T}$ & $\mathrm{G}$ & $\mathrm{C}$ & $\mathrm{G}$ & $\mathrm{C}$ & $\mathrm{A}$ & $\mathrm{G}$ & $\mathrm{T}$ & $\mathrm{A}$ & $\mathrm{G}$ & $\mathrm{A}$ & $\mathrm{C}$ & $\mathrm{G}$ & $\mathrm{A}$ & $\mathrm{G}$ \\
\hline $\begin{array}{c}\text { AFRIC } \\
\text { A }\end{array}$ & $\begin{array}{c}\text { Chagg } \\
a\end{array}$ & $\begin{array}{c}\text { CHA_JK } \\
4143\end{array}$ & $\mathrm{~T}$ & $\mathrm{C}$ & $\mathrm{T}$ & $\mathrm{G}$ & $\mathrm{C}$ & $\mathrm{G}$ & $\mathrm{C}$ & $\mathrm{A}$ & $\mathrm{G}$ & $\mathrm{T}$ & $\mathrm{A}$ & $\mathrm{G}$ & $\mathrm{G}$ & $\mathrm{C}$ & $\mathrm{G}$ & A & $\bar{G}$ \\
\hline $\begin{array}{c}\text { AFRIC } \\
\text { A }\end{array}$ & $\begin{array}{c}\text { Chagg } \\
\text { a }\end{array}$ & $\begin{array}{c}\text { CHA_JK } \\
4144\end{array}$ & $\mathrm{~T}$ & $\mathrm{C}$ & $\mathrm{C}$ & $\mathrm{G}$ & $\mathrm{C}$ & $\mathrm{G}$ & $\mathrm{C}$ & $\mathrm{A}$ & $\mathrm{G}$ & $\mathrm{T}$ & $\mathrm{A}$ & $\mathrm{G}$ & $\mathrm{G}$ & $\mathrm{C}$ & $\mathrm{G}$ & $\mathrm{A}$ & $\mathrm{G}$ \\
\hline $\begin{array}{c}\text { AFRIC } \\
\text { A }\end{array}$ & $\begin{array}{c}\text { Chagg } \\
\text { a }\end{array}$ & $\begin{array}{c}\text { CHA_JK } \\
4144\end{array}$ & $\mathrm{~T}$ & $\mathrm{C}$ & $\mathrm{T}$ & $\mathrm{G}$ & $\mathrm{C}$ & $\mathrm{G}$ & $\mathrm{T}$ & $\mathrm{A}$ & $\mathrm{G}$ & $\mathrm{T}$ & $\mathrm{A}$ & $\mathrm{G}$ & $\mathrm{G}$ & C & $\mathrm{G}$ & $\mathrm{A}$ & $\bar{G}$ \\
\hline $\begin{array}{c}\text { AFRIC } \\
\text { A }\end{array}$ & $\begin{array}{c}\text { Chagg } \\
\text { a }\end{array}$ & $\begin{array}{c}\text { CHA_JK } \\
4145\end{array}$ & $\mathrm{~T}$ & $\mathrm{C}$ & $\mathrm{T}$ & $\mathrm{G}$ & $\mathrm{C}$ & $\mathrm{A}$ & $\mathrm{C}$ & $\mathrm{A}$ & $\mathrm{G}$ & $\mathrm{T}$ & $\mathrm{A}$ & $\mathrm{G}$ & $\mathrm{G}$ & $\mathrm{C}$ & $\mathrm{G}$ & $\mathrm{A}$ & $\bar{G}$ \\
\hline $\begin{array}{c}\text { AFRIC } \\
\text { A }\end{array}$ & $\begin{array}{c}\text { Chagg } \\
\text { a }\end{array}$ & $\begin{array}{c}\text { CHA_JK } \\
41 \overline{45}\end{array}$ & $\mathrm{~T}$ & $\mathrm{C}$ & $\mathrm{T}$ & $\mathrm{G}$ & $\mathrm{C}$ & $\mathrm{G}$ & $\mathrm{C}$ & $\mathrm{A}$ & $\mathrm{G}$ & $\mathrm{T}$ & $\mathrm{A}$ & $\mathrm{G}$ & $\mathrm{A}$ & $\mathrm{C}$ & $\mathrm{G}$ & $\mathrm{A}$ & $\mathrm{G}$ \\
\hline $\begin{array}{c}\text { AFRIC } \\
\text { A }\end{array}$ & $\begin{array}{c}\text { Chagg } \\
\text { a }\end{array}$ & $\begin{array}{c}\text { CHA_JK } \\
4146\end{array}$ & $\mathrm{~T}$ & $\mathrm{C}$ & $\mathrm{T}$ & $\mathrm{G}$ & $\mathrm{C}$ & $\mathrm{G}$ & $\mathrm{C}$ & $\mathrm{A}$ & $\mathrm{G}$ & $\mathrm{T}$ & $\mathrm{A}$ & $\mathrm{G}$ & $\mathrm{G}$ & C & $\mathrm{G}$ & $\mathrm{A}$ & $\bar{G}$ \\
\hline
\end{tabular}

John Wiley 28 Sons, Inc. 


\begin{tabular}{|c|c|c|c|c|c|c|c|c|c|c|c|c|c|c|c|c|c|c|c|}
\hline $\begin{array}{c}\text { AFRIC } \\
\text { A }\end{array}$ & $\begin{array}{c}\text { Chagg } \\
\text { a }\end{array}$ & $\begin{array}{c}\text { CHA_JK } \\
4146\end{array}$ & $\mathrm{~T}$ & $\mathrm{C}$ & $\mathrm{T}$ & $\bar{G}$ & $\mathrm{C}$ & $\mathrm{G}$ & $\mathrm{C}$ & $\mathrm{A}$ & $\mathrm{G}$ & $\mathrm{T}$ & $\overline{\mathrm{A}}$ & $\mathrm{G}$ & $\mathrm{G}$ & $\mathrm{T}$ & $\mathrm{G}$ & $\mathrm{A}$ & $\mathrm{G}$ \\
\hline $\begin{array}{c}\text { AFRIC } \\
\text { A }\end{array}$ & $\begin{array}{c}\text { Chagg } \\
a\end{array}$ & $\begin{array}{c}\text { CHA_JK } \\
41 \overline{47} \\
\end{array}$ & $\mathrm{~T}$ & $\mathrm{C}$ & $\mathrm{T}$ & $\bar{G}$ & $\mathrm{C}$ & $\mathrm{G}$ & $\mathrm{C}$ & $\bar{A}$ & $\mathrm{G}$ & $\mathrm{T}$ & $\mathrm{A}$ & $\mathrm{G}$ & $\mathrm{G}$ & $\mathrm{C}$ & $\mathrm{G}$ & $\mathrm{A}$ & $\mathrm{G}$ \\
\hline $\begin{array}{c}\text { AFRIC } \\
\text { A }\end{array}$ & $\begin{array}{c}\text { Chagg } \\
a\end{array}$ & $\begin{array}{c}\text { CHA_JK } \\
41 \overline{47}\end{array}$ & $\bar{T}$ & $\mathrm{C}$ & $\mathrm{C}$ & $\bar{G}$ & $\mathrm{C}$ & $\mathrm{G}$ & $\mathrm{C}$ & $\overline{\mathrm{A}}$ & $\bar{G}$ & $\mathrm{~T}$ & $\bar{A}$ & $\bar{G}$ & $\bar{G}$ & $\mathrm{C}$ & $\mathrm{G}$ & $\mathrm{A}$ & $\mathrm{G}$ \\
\hline $\begin{array}{c}\text { AFRIC } \\
\text { A }\end{array}$ & $\begin{array}{c}\text { Chagg } \\
a\end{array}$ & $\begin{array}{c}\text { CHA_JK } \\
4148\end{array}$ & $\mathrm{~T}$ & $\mathrm{C}$ & $\mathrm{T}$ & G & $\mathrm{C}$ & $\mathrm{G}$ & $\mathrm{C}$ & $\mathrm{A}$ & $\mathrm{G}$ & $\mathrm{T}$ & A & $\mathrm{G}$ & $\mathrm{G}$ & $\mathrm{C}$ & $\mathrm{G}$ & $\mathrm{A}$ & $\mathrm{G}$ \\
\hline $\begin{array}{c}\text { AFRIC } \\
\text { A }\end{array}$ & $\begin{array}{c}\text { Chagg } \\
\text { a }\end{array}$ & $\begin{array}{c}\text { CHA_JK } \\
4148\end{array}$ & $\mathrm{~T}$ & $\mathrm{C}$ & $\mathrm{T}$ & $\mathrm{G}$ & $\mathrm{C}$ & $\mathrm{G}$ & $\mathrm{C}$ & $\mathrm{A}$ & $\mathrm{G}$ & $\mathrm{T}$ & $\mathrm{A}$ & $\mathrm{G}$ & $\mathrm{G}$ & $\mathrm{C}$ & $\mathrm{G}$ & $\mathrm{A}$ & $\bar{G}$ \\
\hline $\begin{array}{c}\text { AFRIC } \\
\text { A }\end{array}$ & $\begin{array}{c}\text { Chagg } \\
\mathrm{a}\end{array}$ & $\begin{array}{c}\text { CHA_JK } \\
4149 \\
\end{array}$ & $\mathrm{~T}$ & $\mathrm{C}$ & $\mathrm{T}$ & $\mathrm{G}$ & $\mathrm{C}$ & $\mathrm{G}$ & $\mathrm{C}$ & $\mathrm{A}$ & $\mathrm{G}$ & $\mathrm{T}$ & $\mathrm{A}$ & $\mathrm{G}$ & $G$ & $\mathrm{C}$ & $\mathrm{G}$ & $\mathrm{C}$ & $\bar{G}$ \\
\hline $\begin{array}{c}\text { AFRIC } \\
\text { A }\end{array}$ & $\begin{array}{c}\text { Chagg } \\
a\end{array}$ & $\begin{array}{c}\text { CHA_JK } \\
4149\end{array}$ & $\mathrm{~T}$ & $\mathrm{C}$ & $\mathrm{T}$ & $\mathrm{G}$ & $\mathrm{C}$ & $\mathrm{G}$ & $\mathrm{C}$ & $\mathrm{A}$ & $\mathrm{G}$ & $\mathrm{T}$ & $\mathrm{A}$ & $\mathrm{G}$ & $\mathrm{G}$ & $\mathrm{C}$ & $\mathrm{G}$ & $\bar{A}$ & $\bar{G}$ \\
\hline $\begin{array}{c}\text { AFRIC } \\
\text { A }\end{array}$ & $\begin{array}{c}\text { Chagg } \\
\text { a }\end{array}$ & $\begin{array}{c}\text { CHA_JK } \\
41 \overline{50}\end{array}$ & $\mathrm{~T}$ & $\mathrm{C}$ & $\mathrm{T}$ & $\mathrm{G}$ & $\mathrm{C}$ & $\mathrm{G}$ & $\mathrm{C}$ & $\mathrm{A}$ & $\mathrm{G}$ & $\mathrm{T}$ & $\mathrm{A}$ & $\mathrm{G}$ & $\mathrm{G}$ & $\mathrm{C}$ & $\mathrm{G}$ & $\mathrm{A}$ & $\bar{G}$ \\
\hline $\begin{array}{c}\text { AFRIC } \\
\text { A }\end{array}$ & $\begin{array}{c}\text { Chagg } \\
\text { a }\end{array}$ & $\begin{array}{c}\text { CHA_JK } \\
4150\end{array}$ & $\mathrm{~T}$ & $\mathrm{C}$ & $\mathrm{C}$ & $\mathrm{G}$ & $\mathrm{C}$ & $\mathrm{G}$ & $\mathrm{C}$ & $\mathrm{A}$ & $\mathrm{G}$ & $\mathrm{T}$ & $\mathrm{A}$ & $\mathrm{G}$ & $\mathrm{G}$ & $\mathrm{C}$ & $\mathrm{G}$ & $\mathrm{A}$ & $\bar{G}$ \\
\hline $\begin{array}{c}\text { AFRIC } \\
\text { A }\end{array}$ & $\begin{array}{c}\text { Chagg } \\
\text { a }\end{array}$ & $\begin{array}{c}\text { CHA_JK } \\
4151\end{array}$ & $\mathrm{~T}$ & $\mathrm{C}$ & $\mathrm{T}$ & G & $\mathrm{C}$ & $\mathrm{G}$ & $\mathrm{C}$ & $\mathrm{A}$ & $\mathrm{G}$ & $\mathrm{T}$ & $\mathrm{A}$ & $\mathrm{G}$ & $\mathrm{G}$ & $\mathrm{C}$ & $\mathrm{G}$ & $\mathrm{A}$ & G \\
\hline $\begin{array}{c}\text { AFRIC } \\
\text { A }\end{array}$ & $\begin{array}{c}\text { Chagg } \\
\text { a }\end{array}$ & $\begin{array}{c}\text { CHA_JK } \\
41 \overline{51}\end{array}$ & $\mathrm{~T}$ & $\bar{C}$ & $\mathrm{~T}$ & $\bar{G}$ & $\mathrm{C}$ & $\bar{G}$ & $\mathrm{C}$ & $\mathrm{A}$ & $\mathrm{G}$ & $\mathrm{T}$ & $\mathrm{A}$ & $\mathrm{G}$ & $\bar{G}$ & $\mathrm{C}$ & $\mathrm{G}$ & $\mathrm{A}$ & $\overline{\mathrm{G}}$ \\
\hline $\begin{array}{c}\text { AFRIC } \\
\text { A }\end{array}$ & $\begin{array}{c}\text { Chagg } \\
a\end{array}$ & $\begin{array}{c}\text { CHA_JK } \\
4152 \\
\end{array}$ & $\mathrm{~T}$ & $\mathrm{C}$ & $\mathrm{T}$ & $\mathrm{G}$ & $\mathrm{C}$ & $\mathrm{G}$ & $\mathrm{C}$ & $\bar{A}$ & $G$ & $\mathrm{~T}$ & $\mathrm{~A}$ & $\mathrm{G}$ & $\mathrm{G}$ & $\mathrm{C}$ & $\mathrm{G}$ & $\mathrm{A}$ & $\mathrm{G}$ \\
\hline $\begin{array}{c}\text { AFRIC } \\
\text { A }\end{array}$ & $\begin{array}{c}\text { Chagg } \\
\text { a }\end{array}$ & $\begin{array}{c}\text { CHA_JK } \\
4152\end{array}$ & $\mathrm{C}$ & $\mathrm{C}$ & $\mathrm{T}$ & $\bar{G}$ & $\mathrm{C}$ & $\mathrm{G}$ & $\mathrm{C}$ & $\bar{A}$ & $\mathrm{G}$ & $\mathrm{T}$ & $\mathrm{A}$ & $\mathrm{G}$ & $\mathrm{G}$ & $\mathrm{C}$ & $\mathrm{G}$ & $\mathrm{A}$ & $\mathrm{G}$ \\
\hline $\begin{array}{c}\text { AFRIC } \\
\text { A }\end{array}$ & $\begin{array}{c}\text { Chagg } \\
\text { a }\end{array}$ & $\begin{array}{c}\text { CHA_JK } \\
4153\end{array}$ & $\mathrm{~T}$ & $\bar{C}$ & $\mathrm{~T}$ & $\bar{G}$ & $\mathrm{C}$ & $\bar{G}$ & $\mathrm{C}$ & $\mathrm{A}$ & $\mathrm{G}$ & $\mathrm{T}$ & $\mathrm{A}$ & $\mathrm{G}$ & $\bar{G}$ & $\mathrm{C}$ & $\mathrm{G}$ & $\mathrm{A}$ & $\overline{\mathrm{G}}$ \\
\hline $\begin{array}{c}\text { AFRIC } \\
\text { A }\end{array}$ & $\begin{array}{c}\text { Chagg } \\
a\end{array}$ & $\begin{array}{c}\text { CHA_JK } \\
4153 \\
\end{array}$ & $\mathrm{~T}$ & $\mathrm{C}$ & $\mathrm{T}$ & $\bar{G}$ & $\mathrm{C}$ & $\bar{G}$ & $\mathrm{C}$ & $\overline{\mathrm{A}}$ & $\mathrm{G}$ & $\mathrm{T}$ & $\mathrm{A}$ & $\mathrm{G}$ & $\mathrm{G}$ & $\mathrm{C}$ & $\mathrm{G}$ & $\mathrm{A}$ & $\mathrm{G}$ \\
\hline $\begin{array}{c}\text { AFRIC } \\
\text { A }\end{array}$ & $\begin{array}{c}\text { Chagg } \\
\text { a }\end{array}$ & $\begin{array}{c}\text { CHA_JK } \\
4154\end{array}$ & $\mathrm{~T}$ & $\mathrm{C}$ & $\mathrm{T}$ & $\bar{G}$ & $\mathrm{C}$ & $\mathrm{G}$ & $\mathrm{C}$ & $\mathrm{A}$ & $\mathrm{G}$ & $\mathrm{T}$ & $\mathrm{A}$ & $\mathrm{G}$ & $\mathrm{G}$ & $\mathrm{C}$ & $\mathrm{G}$ & $\mathrm{A}$ & $\mathrm{G}$ \\
\hline $\begin{array}{c}\text { AFRIC } \\
\text { A }\end{array}$ & $\begin{array}{c}\text { Chagg } \\
a\end{array}$ & $\begin{array}{c}\text { CHA_JK } \\
41 \overline{54}\end{array}$ & $\mathrm{~T}$ & $\mathrm{C}$ & $\mathrm{T}$ & $\mathrm{G}$ & $\mathrm{C}$ & $\bar{G}$ & $\mathrm{C}$ & $\mathrm{A}$ & $\mathrm{G}$ & $\mathrm{T}$ & $\mathrm{A}$ & $\mathrm{G}$ & $\mathrm{G}$ & $\mathrm{C}$ & $\mathrm{G}$ & $\mathrm{A}$ & $\bar{G}$ \\
\hline $\begin{array}{c}\text { AFRIC } \\
\text { A }\end{array}$ & $\begin{array}{c}\text { Chagg } \\
\mathrm{a}\end{array}$ & $\begin{array}{c}\text { CHA_JK } \\
4155\end{array}$ & $\mathrm{~T}$ & $\mathrm{C}$ & $\mathrm{T}$ & G & $\mathrm{C}$ & $\mathrm{G}$ & $\mathrm{C}$ & $\overline{\mathrm{A}}$ & $\mathrm{G}$ & $\mathrm{T}$ & $\mathrm{A}$ & $\mathrm{G}$ & $\mathrm{G}$ & $\mathrm{C}$ & $\mathrm{G}$ & $\mathrm{A}$ & $\mathrm{G}$ \\
\hline $\begin{array}{c}\text { AFRIC } \\
\text { A }\end{array}$ & $\begin{array}{c}\text { Chagg } \\
\mathrm{a}\end{array}$ & $\begin{array}{c}\text { CHA_JK } \\
4155 \\
\end{array}$ & $\mathrm{~T}$ & $\mathrm{C}$ & $\mathrm{C}$ & G & $\mathrm{C}$ & $\mathrm{G}$ & $\mathrm{C}$ & $\mathrm{A}$ & $\mathrm{G}$ & $\mathrm{T}$ & A & $\mathrm{G}$ & G & $\mathrm{C}$ & $\mathrm{G}$ & $\mathrm{A}$ & $\mathrm{G}$ \\
\hline $\begin{array}{c}\text { AFRIC } \\
\text { A }\end{array}$ & $\begin{array}{c}\text { Chagg } \\
\text { a }\end{array}$ & $\begin{array}{c}\text { CHA_JK } \\
4156\end{array}$ & $\mathrm{~T}$ & $\mathrm{C}$ & $\mathrm{T}$ & G & $\mathrm{C}$ & $\mathrm{G}$ & $\mathrm{C}$ & $\mathrm{A}$ & $\mathrm{G}$ & $\mathrm{T}$ & A & $\mathrm{G}$ & G & $\mathrm{C}$ & $\mathrm{G}$ & $\mathrm{A}$ & $\mathrm{G}$ \\
\hline $\begin{array}{c}\text { AFRIC } \\
\text { A }\end{array}$ & $\begin{array}{c}\text { Chagg } \\
a\end{array}$ & $\begin{array}{c}\text { CHA_JK } \\
4156\end{array}$ & $\mathrm{~T}$ & $\mathrm{C}$ & $\mathrm{C}$ & G & $\mathrm{C}$ & $\mathrm{G}$ & $\mathrm{C}$ & A & $\mathrm{G}$ & $\mathrm{T}$ & A & $\mathrm{G}$ & G & $\mathrm{C}$ & $\mathrm{G}$ & $\mathrm{A}$ & $\mathrm{G}$ \\
\hline $\begin{array}{c}\text { AFRIC } \\
\text { A }\end{array}$ & Yoruba & $\begin{array}{c}\text { YRB_JK3 } \\
878\end{array}$ & $\mathrm{C}$ & $\mathrm{C}$ & $\mathrm{T}$ & G & $\mathrm{C}$ & $\mathrm{G}$ & $\mathrm{C}$ & A & $\mathrm{A}$ & $\mathrm{T}$ & A & $\mathrm{G}$ & G & $\mathrm{C}$ & $\mathrm{G}$ & $\mathrm{A}$ & $\mathrm{G}$ \\
\hline $\begin{array}{c}\text { AFRIC } \\
\text { A }\end{array}$ & Yoruba & $\begin{array}{c}\text { YRB_JK3 } \\
878\end{array}$ & $\mathrm{~T}$ & $\mathrm{C}$ & $\mathrm{T}$ & G & $\mathrm{C}$ & $\mathrm{G}$ & $\mathrm{C}$ & $\mathrm{A}$ & $\mathrm{G}$ & $\mathrm{T}$ & $\mathrm{A}$ & $\mathrm{G}$ & $\mathrm{G}$ & $\mathrm{C}$ & $\mathrm{G}$ & $\mathrm{A}$ & G \\
\hline
\end{tabular}




\begin{tabular}{|c|c|c|c|c|c|c|c|c|c|c|c|c|c|c|c|c|c|c|c|}
\hline $\begin{array}{c}\text { AFRIC } \\
\text { A }\end{array}$ & Yoruba & $\begin{array}{c}\text { YRB_JK3 } \\
879\end{array}$ & $\mathrm{~T}$ & $\mathrm{C}$ & $\mathrm{T}$ & $\mathrm{G}$ & $\mathrm{C}$ & $\mathrm{G}$ & $\mathrm{C}$ & $\bar{A}$ & $\mathrm{G}$ & $\mathrm{T}$ & $\mathrm{A}$ & $\bar{G}$ & $\mathrm{G}$ & $\mathrm{C}$ & $\mathrm{G}$ & $\mathrm{A}$ & $\bar{G}$ \\
\hline $\begin{array}{c}\text { AFRIC } \\
\text { A }\end{array}$ & Yoruba & $\begin{array}{c}\text { YRB_JK3 } \\
879 \\
\end{array}$ & $\mathrm{~T}$ & C & $\mathrm{T}$ & G & $\mathrm{C}$ & G & $\mathrm{C}$ & $\mathrm{A}$ & G & $\mathrm{T}$ & $\mathrm{A}$ & G & $\mathrm{G}$ & $\mathrm{C}$ & G & $\mathrm{A}$ & $\mathrm{G}$ \\
\hline $\begin{array}{c}\text { AFRIC } \\
\text { A }\end{array}$ & Yoruba & $\begin{array}{c}\text { YRB_JK3 } \\
880\end{array}$ & $\mathrm{~T}$ & $\mathrm{C}$ & $\mathrm{T}$ & $\mathrm{G}$ & $\bar{C}$ & $\mathrm{G}$ & $\mathrm{C}$ & $\mathrm{A}$ & $\mathrm{G}$ & $\mathrm{T}$ & $\mathrm{A}$ & $\mathrm{G}$ & $\mathrm{G}$ & $\mathrm{C}$ & $\mathrm{G}$ & $\mathrm{A}$ & $\mathrm{G}$ \\
\hline $\begin{array}{c}\text { AFRIC } \\
\text { A }\end{array}$ & Yoruba & $\begin{array}{c}\text { YRB_JK3 } \\
880 \\
\end{array}$ & $\mathrm{~T}$ & $\mathrm{C}$ & $\mathrm{T}$ & $\mathrm{G}$ & $\mathrm{C}$ & $\mathrm{G}$ & $\mathrm{C}$ & $\mathrm{A}$ & $\mathrm{G}$ & $\mathrm{T}$ & C & G & $\mathrm{A}$ & C & G & $\mathrm{A}$ & $\mathrm{G}$ \\
\hline $\begin{array}{c}\text { AFRIC } \\
\text { A }\end{array}$ & Yoruba & $\begin{array}{c}\text { YRB_JK3 } \\
881\end{array}$ & $\mathrm{~T}$ & $\mathrm{C}$ & $\mathrm{T}$ & $\mathrm{G}$ & $\mathrm{C}$ & $\mathrm{G}$ & $\mathrm{C}$ & $\mathrm{A}$ & $\mathrm{G}$ & $\mathrm{T}$ & $\mathrm{A}$ & $\mathrm{G}$ & $\mathrm{G}$ & $\mathrm{C}$ & $\mathrm{G}$ & $\mathrm{A}$ & $\mathrm{G}$ \\
\hline $\begin{array}{c}\text { AFRIC } \\
\text { A }\end{array}$ & Yoruba & $\begin{array}{c}\text { YRB_JK3 } \\
881\end{array}$ & $\mathrm{~T}$ & $\mathrm{C}$ & $\mathrm{T}$ & $\mathrm{G}$ & $\mathrm{C}$ & $\mathrm{G}$ & $\mathrm{C}$ & A & $\mathrm{G}$ & $\mathrm{T}$ & $\mathrm{A}$ & $\mathrm{G}$ & $\mathrm{G}$ & $\mathrm{C}$ & $\mathrm{G}$ & A & $\mathrm{G}$ \\
\hline $\begin{array}{c}\text { AFRIC } \\
\text { A }\end{array}$ & Yoruba & $\begin{array}{c}\text { YRB_JK3 } \\
882\end{array}$ & $\mathrm{C}$ & $\mathrm{C}$ & $\mathrm{T}$ & $\mathrm{G}$ & $\mathrm{C}$ & $\mathrm{G}$ & $\mathrm{C}$ & $\mathrm{A}$ & $\mathrm{G}$ & $\mathrm{T}$ & $\mathrm{A}$ & $\mathrm{A}$ & $\mathrm{G}$ & $\mathrm{C}$ & $\mathrm{G}$ & $\mathrm{A}$ & $\mathrm{G}$ \\
\hline $\begin{array}{c}\text { AFRIC } \\
\text { A }\end{array}$ & Yoruba & $\begin{array}{c}\text { YRB_JK3 } \\
882 \\
\end{array}$ & $\mathrm{~T}$ & $\mathrm{C}$ & $\mathrm{T}$ & $\mathrm{G}$ & $\mathrm{C}$ & G & $\mathrm{C}$ & $\mathrm{A}$ & $\mathrm{G}$ & $\mathrm{T}$ & $\mathrm{A}$ & G & $\mathrm{G}$ & $\mathrm{C}$ & $\mathrm{G}$ & $\mathrm{A}$ & G \\
\hline $\begin{array}{c}\text { AFRIC } \\
\text { A }\end{array}$ & Yoruba & $\begin{array}{c}\text { YRB_JK3 } \\
883\end{array}$ & $\mathrm{~T}$ & $\mathrm{C}$ & $\mathrm{T}$ & $\mathrm{G}$ & $\mathrm{C}$ & $\mathrm{G}$ & $\mathrm{C}$ & A & $\mathrm{G}$ & $\mathrm{T}$ & $\mathrm{A}$ & $\mathrm{G}$ & $\mathrm{G}$ & $\mathrm{C}$ & $\mathrm{G}$ & A & G \\
\hline $\begin{array}{c}\text { AFRIC } \\
\text { A }\end{array}$ & Yoruba & $\begin{array}{c}\text { YRB_JK3 } \\
883\end{array}$ & $\mathrm{~T}$ & $\mathrm{C}$ & $\mathrm{T}$ & $\mathrm{G}$ & $\mathrm{C}$ & $G$ & $\mathrm{C}$ & $\mathrm{A}$ & $\mathrm{G}$ & $\mathrm{T}$ & $\mathrm{A}$ & G & $\mathrm{G}$ & C & $\mathrm{G}$ & $\mathrm{C}$ & $\bar{G}$ \\
\hline $\begin{array}{c}\text { AFRIC } \\
\text { A }\end{array}$ & Yoruba & $\begin{array}{c}\text { YRB_JK3 } \\
884 \\
\end{array}$ & $\mathrm{~T}$ & $\mathrm{C}$ & $\mathrm{T}$ & $\mathrm{G}$ & $\mathrm{C}$ & $\mathrm{G}$ & $\mathrm{C}$ & $\mathrm{A}$ & $\mathrm{G}$ & $\mathrm{T}$ & $\mathrm{A}$ & $\mathrm{G}$ & $\mathrm{G}$ & $\mathrm{C}$ & $\mathrm{G}$ & $\mathrm{A}$ & $\bar{G}$ \\
\hline $\begin{array}{c}\text { AFRIC } \\
\text { A }\end{array}$ & Yoruba & $\begin{array}{c}\text { YRB_JK3 } \\
884\end{array}$ & $\mathrm{~T}$ & $\mathrm{C}$ & $\mathrm{T}$ & $\mathrm{G}$ & $\mathrm{C}$ & $\mathrm{G}$ & $\mathrm{C}$ & $\mathrm{A}$ & $\mathrm{G}$ & $\mathrm{T}$ & $\mathrm{A}$ & $\mathrm{G}$ & $\mathrm{A}$ & $\mathrm{C}$ & $\mathrm{G}$ & A & $\mathrm{G}$ \\
\hline $\begin{array}{c}\text { AFRIC } \\
\text { A }\end{array}$ & Yoruba & $\begin{array}{c}\text { YRB_JK3 } \\
885\end{array}$ & $\mathrm{~T}$ & $\mathrm{C}$ & $\mathrm{T}$ & $\mathrm{G}$ & $\mathrm{C}$ & $\mathrm{G}$ & $\mathrm{C}$ & $\mathrm{A}$ & $\mathrm{G}$ & $\mathrm{T}$ & $\mathrm{A}$ & $\mathrm{G}$ & $\mathrm{G}$ & $\mathrm{C}$ & $\mathrm{G}$ & $\mathrm{A}$ & $\bar{G}$ \\
\hline $\begin{array}{c}\text { AFRIC } \\
\text { A }\end{array}$ & Yoruba & $\begin{array}{c}\text { YRB_JK3 } \\
885\end{array}$ & $\mathrm{~T}$ & $\mathrm{C}$ & $\mathrm{T}$ & $\mathrm{G}$ & $\mathrm{C}$ & $\mathrm{G}$ & $\mathrm{C}$ & $\mathrm{A}$ & $\mathrm{G}$ & $\mathrm{T}$ & $\mathrm{A}$ & $\bar{G}$ & $\mathrm{~A}$ & $\mathrm{C}$ & $\mathrm{G}$ & $\bar{A}$ & $\overline{\mathrm{G}}$ \\
\hline $\begin{array}{c}\text { AFRIC } \\
\text { A }\end{array}$ & Yoruba & $\begin{array}{c}\text { YRB_JK3 } \\
886\end{array}$ & $\mathrm{~T}$ & $\mathrm{C}$ & $\mathrm{T}$ & $\mathrm{G}$ & $\mathrm{C}$ & $\mathrm{G}$ & $\mathrm{C}$ & $\mathrm{A}$ & $\mathrm{G}$ & $\mathrm{T}$ & $\mathrm{A}$ & $\mathrm{G}$ & $\mathrm{G}$ & $\bar{C}$ & $\mathrm{G}$ & $\mathrm{A}$ & $\bar{G}$ \\
\hline $\begin{array}{c}\text { AFRIC } \\
\text { A }\end{array}$ & Yoruba & $\begin{array}{c}\text { YRB_JK3 } \\
886\end{array}$ & $\mathrm{~T}$ & $\mathrm{C}$ & $\mathrm{T}$ & $\mathrm{G}$ & $\mathrm{C}$ & $\mathrm{A}$ & $\mathrm{C}$ & A & $\mathrm{G}$ & $\mathrm{T}$ & $\bar{A}$ & $\mathrm{G}$ & $\mathrm{G}$ & $\mathrm{C}$ & $\mathrm{G}$ & $\mathrm{A}$ & $\mathrm{G}$ \\
\hline $\begin{array}{c}\text { AFRIC } \\
\text { A }\end{array}$ & Yoruba & $\begin{array}{c}\text { YRB_JK3 } \\
887\end{array}$ & $\mathrm{~T}$ & $\mathrm{C}$ & $\mathrm{T}$ & $\mathrm{G}$ & $\mathrm{C}$ & $\bar{G}$ & $\mathrm{C}$ & $\mathrm{A}$ & $\mathrm{G}$ & $\mathrm{T}$ & $\mathrm{A}$ & $\mathrm{G}$ & $\mathrm{G}$ & $\mathrm{C}$ & $\mathrm{G}$ & $\mathrm{C}$ & $\mathrm{G}$ \\
\hline $\begin{array}{c}\text { AFRIC } \\
\text { A }\end{array}$ & Yoruba & $\begin{array}{c}\text { YRB_JK3 } \\
887\end{array}$ & $\mathrm{~T}$ & $\mathrm{C}$ & $\mathrm{T}$ & $\mathrm{G}$ & $\mathrm{C}$ & $\mathrm{G}$ & $\mathrm{C}$ & $\mathrm{A}$ & $\mathrm{G}$ & $\mathrm{T}$ & $\mathrm{A}$ & $\mathrm{G}$ & $\mathrm{A}$ & $\mathrm{C}$ & $\mathrm{G}$ & A & $\mathrm{G}$ \\
\hline $\begin{array}{c}\text { AFRIC } \\
\text { A }\end{array}$ & Yoruba & $\begin{array}{c}\text { YRB_JK3 } \\
888\end{array}$ & $\mathrm{~T}$ & $\mathrm{C}$ & $\mathrm{T}$ & $\mathrm{G}$ & $\mathrm{C}$ & $\mathrm{G}$ & $\mathrm{C}$ & $\mathrm{A}$ & $\mathrm{G}$ & $\mathrm{T}$ & $\mathrm{A}$ & $\mathrm{G}$ & $\mathrm{G}$ & $\mathrm{C}$ & G & $\mathrm{A}$ & $\mathrm{G}$ \\
\hline $\begin{array}{c}\text { AFRIC } \\
\text { A }\end{array}$ & Yoruba & $\begin{array}{c}\text { YRB_JK3 } \\
888 \\
\end{array}$ & $\mathrm{~T}$ & $\mathrm{C}$ & $\mathrm{T}$ & $\mathrm{G}$ & $\mathrm{C}$ & $\mathrm{G}$ & $\mathrm{C}$ & A & G & $\mathrm{T}$ & $\mathrm{A}$ & $\mathrm{G}$ & $\mathrm{G}$ & $\mathrm{C}$ & G & $\mathrm{A}$ & G \\
\hline $\begin{array}{c}\text { AFRIC } \\
\text { A }\end{array}$ & Yoruba & $\begin{array}{c}\text { YRB_JK3 } \\
889 \\
\end{array}$ & $\mathrm{~T}$ & $\mathrm{C}$ & $\mathrm{T}$ & $\mathrm{G}$ & $\mathrm{C}$ & $\mathrm{G}$ & $\mathrm{C}$ & $\mathrm{A}$ & $\mathrm{G}$ & $\mathrm{T}$ & $\mathrm{A}$ & $\mathrm{G}$ & $\mathrm{G}$ & $\mathrm{C}$ & $\mathrm{G}$ & A & G \\
\hline $\begin{array}{c}\text { AFRIC } \\
\text { A }\end{array}$ & Yoruba & $\begin{array}{c}\text { YRB_JK3 } \\
889\end{array}$ & $\mathrm{~T}$ & $\mathrm{C}$ & $\mathrm{T}$ & $\mathrm{G}$ & $\mathrm{C}$ & $\mathrm{G}$ & $\mathrm{C}$ & $\mathrm{A}$ & $\mathrm{G}$ & $\mathrm{T}$ & $\mathrm{A}$ & $\mathrm{G}$ & $\mathrm{G}$ & $\mathrm{C}$ & $\mathrm{G}$ & A & G \\
\hline $\begin{array}{c}\text { AFRIC } \\
\text { A }\end{array}$ & Yoruba & $\begin{array}{c}\text { YRB_JK3 } \\
891\end{array}$ & $\mathrm{~T}$ & $\mathrm{C}$ & $\mathrm{T}$ & $\mathrm{G}$ & $\mathrm{C}$ & $\mathrm{G}$ & $\mathrm{C}$ & A & G & $\mathrm{T}$ & $\mathrm{A}$ & $\mathrm{G}$ & $\mathrm{A}$ & $\mathrm{C}$ & G & $\mathrm{A}$ & G \\
\hline
\end{tabular}

John Wiley 30 Sons, Inc. 


\begin{tabular}{|c|c|c|c|c|c|c|c|c|c|c|c|c|c|c|c|c|c|c|c|}
\hline $\begin{array}{c}\text { AFRIC } \\
\text { A }\end{array}$ & Yoruba & $\begin{array}{c}\text { YRB_JK3 } \\
891\end{array}$ & $\mathrm{~T}$ & $\mathrm{C}$ & $\mathrm{C}$ & $\mathrm{G}$ & $\mathrm{C}$ & $\mathrm{G}$ & $\mathrm{C}$ & $\mathrm{A}$ & $\mathrm{G}$ & $\mathrm{T}$ & $\overline{\mathrm{A}}$ & $\mathrm{G}$ & $\mathrm{G}$ & $\mathrm{C}$ & $\mathrm{G}$ & $\mathrm{A}$ & $\mathrm{G}$ \\
\hline $\begin{array}{c}\text { AFRIC } \\
\text { A }\end{array}$ & Yoruba & $\begin{array}{c}\text { YRB_JK3 } \\
892 \\
\end{array}$ & $\mathrm{~T}$ & $\mathrm{C}$ & $\mathrm{T}$ & $\mathrm{G}$ & $\bar{C}$ & $\mathrm{G}$ & $\mathrm{C}$ & $\bar{A}$ & $\mathrm{G}$ & $\mathrm{T}$ & A & $\mathrm{G}$ & $\mathrm{A}$ & $\mathrm{C}$ & $\mathrm{G}$ & $\mathrm{A}$ & $\mathrm{G}$ \\
\hline $\begin{array}{c}\text { AFRIC } \\
\text { A }\end{array}$ & Yoruba & $\begin{array}{c}\text { YRB_JK3 } \\
892 \\
\end{array}$ & $\bar{T}$ & $\mathrm{C}$ & $\mathrm{T}$ & $\mathrm{G}$ & $\mathrm{C}$ & $\mathrm{G}$ & $\mathrm{C}$ & $\overline{\mathrm{A}}$ & $\mathrm{G}$ & $\mathrm{T}$ & $\bar{A}$ & $\bar{G}$ & $\bar{A}$ & $\mathrm{C}$ & $\mathrm{G}$ & $\mathrm{A}$ & $\mathrm{G}$ \\
\hline $\begin{array}{c}\text { AFRIC } \\
\text { A }\end{array}$ & Yoruba & $\begin{array}{c}\text { YRB_JK3 } \\
893\end{array}$ & $\mathrm{~T}$ & $\mathrm{C}$ & $\mathrm{T}$ & $\mathrm{G}$ & $\mathrm{C}$ & $\mathrm{G}$ & $\mathrm{C}$ & $\mathrm{A}$ & $\mathrm{G}$ & $\mathrm{T}$ & $\mathrm{A}$ & $\mathrm{G}$ & $\mathrm{G}$ & $\mathrm{C}$ & $\mathrm{G}$ & $\mathrm{A}$ & $\mathrm{G}$ \\
\hline $\begin{array}{c}\text { AFRIC } \\
\text { A }\end{array}$ & Yoruba & $\begin{array}{c}\text { YRB_JK3 } \\
893\end{array}$ & $\mathrm{~T}$ & $\mathrm{C}$ & $\mathrm{T}$ & $\mathrm{G}$ & $\mathrm{C}$ & $\mathrm{G}$ & $\mathrm{C}$ & A & $\mathrm{G}$ & $\mathrm{T}$ & $\mathrm{A}$ & $\mathrm{G}$ & $\mathrm{G}$ & $\mathrm{C}$ & $\mathrm{G}$ & $\mathrm{A}$ & $\bar{G}$ \\
\hline $\begin{array}{c}\text { AFRIC } \\
\text { A }\end{array}$ & Yoruba & $\begin{array}{c}\text { YRB_JK3 } \\
894\end{array}$ & $\mathrm{~T}$ & $\mathrm{C}$ & $\mathrm{T}$ & G & $\mathrm{C}$ & $\mathrm{G}$ & $\mathrm{C}$ & $\mathrm{A}$ & $\mathrm{G}$ & $\mathrm{T}$ & $\mathrm{A}$ & $\mathrm{G}$ & $G$ & $\mathrm{C}$ & $\mathrm{G}$ & $\mathrm{A}$ & $\mathrm{G}$ \\
\hline $\begin{array}{c}\text { AFRIC } \\
\text { A }\end{array}$ & Yoruba & \begin{tabular}{c|} 
YRB_JK3 \\
894
\end{tabular} & $\mathrm{~T}$ & $\mathrm{C}$ & $\mathrm{T}$ & $\mathrm{G}$ & $\mathrm{C}$ & $\mathrm{G}$ & $\mathrm{C}$ & $\mathrm{A}$ & $\mathrm{G}$ & $\mathrm{T}$ & $\bar{A}$ & $\mathrm{G}$ & $\mathrm{G}$ & $\mathrm{C}$ & $\mathrm{G}$ & $\bar{A}$ & $\bar{G}$ \\
\hline $\begin{array}{c}\text { AFRIC } \\
\text { A }\end{array}$ & Yoruba & $\begin{array}{c}\text { YRB_JK3 } \\
845\end{array}$ & $\mathrm{~T}$ & $\mathrm{C}$ & $\mathrm{T}$ & $\mathrm{G}$ & $\mathrm{C}$ & $\mathrm{G}$ & $\mathrm{C}$ & A & $\mathrm{G}$ & $\mathrm{T}$ & $\mathrm{A}$ & $\mathrm{G}$ & $\mathrm{G}$ & $\mathrm{C}$ & $\mathrm{G}$ & $\mathrm{A}$ & $\bar{G}$ \\
\hline $\begin{array}{c}\text { AFRIC } \\
\text { A }\end{array}$ & Yoruba & $\begin{array}{c}\text { YRB_JK3 } \\
845\end{array}$ & $\mathrm{~T}$ & $\mathrm{C}$ & $\mathrm{T}$ & $\mathrm{G}$ & $\mathrm{C}$ & $\mathrm{G}$ & $\mathrm{C}$ & $\mathrm{A}$ & $\mathrm{G}$ & $\mathrm{T}$ & $\overline{\mathrm{A}}$ & $\mathrm{A}$ & $\mathrm{G}$ & $\mathrm{C}$ & $\mathrm{G}$ & $\mathrm{A}$ & G \\
\hline $\begin{array}{c}\text { AFRIC } \\
\text { A }\end{array}$ & Yoruba & $\begin{array}{c}\text { YRB_JK3 } \\
846\end{array}$ & $\mathrm{~T}$ & $\mathrm{C}$ & $\mathrm{T}$ & $\mathrm{G}$ & $\mathrm{C}$ & $\mathrm{G}$ & $\mathrm{C}$ & $\mathrm{A}$ & $\mathrm{G}$ & $\mathrm{T}$ & $\mathrm{A}$ & $\mathrm{G}$ & $\mathrm{G}$ & $\mathrm{C}$ & $\mathrm{G}$ & $\mathrm{A}$ & G \\
\hline $\begin{array}{c}\text { AFRIC } \\
\text { A }\end{array}$ & Yoruba & $\begin{array}{c}\text { YRB_JK3 } \\
846\end{array}$ & $\mathrm{~T}$ & $\mathrm{C}$ & $\bar{T}$ & $\mathrm{G}$ & $\mathrm{C}$ & $\bar{G}$ & $\mathrm{C}$ & $\mathrm{A}$ & $\mathrm{G}$ & $\mathrm{T}$ & $\mathrm{A}$ & $\mathrm{G}$ & $\bar{G}$ & $\mathrm{C}$ & $\mathrm{G}$ & $\mathrm{A}$ & $\overline{\mathrm{G}}$ \\
\hline $\begin{array}{c}\text { AFRIC } \\
\text { A }\end{array}$ & Yoruba & $\begin{array}{c}\text { YRB_JK3 } \\
847\end{array}$ & $\mathrm{C}$ & $\mathrm{C}$ & $\mathrm{T}$ & G & $\mathrm{C}$ & $\mathrm{G}$ & $\mathrm{C}$ & $\overline{\mathrm{A}}$ & $G$ & $\mathrm{~T}$ & A & $\mathrm{A}$ & $\mathrm{G}$ & $\mathrm{C}$ & $\mathrm{G}$ & $\mathrm{A}$ & $\mathrm{G}$ \\
\hline $\begin{array}{c}\text { AFRIC } \\
\text { A }\end{array}$ & Yoruba & $\begin{array}{c}\text { YRB_JK3 } \\
847\end{array}$ & $\mathrm{~T}$ & $\mathrm{C}$ & $\mathrm{T}$ & $\mathrm{G}$ & $\mathrm{C}$ & $\mathrm{G}$ & $\mathrm{C}$ & $\bar{A}$ & $\mathrm{G}$ & $\mathrm{T}$ & $\bar{A}$ & $\mathrm{G}$ & $\mathrm{G}$ & $\mathrm{C}$ & $\mathrm{G}$ & $\mathrm{A}$ & $\mathrm{G}$ \\
\hline $\begin{array}{c}\text { AFRIC } \\
\text { A }\end{array}$ & Yoruba & $\begin{array}{c}\text { YRB_JK3 } \\
848\end{array}$ & $\mathrm{~T}$ & $\mathrm{C}$ & $\mathrm{T}$ & $\mathrm{G}$ & $\mathrm{C}$ & $\bar{G}$ & $\mathrm{C}$ & $\mathrm{A}$ & $\mathrm{G}$ & $\mathrm{T}$ & $\mathrm{A}$ & $\mathrm{G}$ & $\bar{G}$ & $\mathrm{C}$ & $\mathrm{G}$ & $\mathrm{A}$ & $\overline{\mathrm{G}}$ \\
\hline $\begin{array}{c}\text { AFRIC } \\
\text { A }\end{array}$ & Yoruba & $\begin{array}{c}\text { YRB_JK3 } \\
848 \\
\end{array}$ & $\mathrm{~T}$ & $\mathrm{C}$ & $\mathrm{T}$ & $\mathrm{G}$ & $\mathrm{C}$ & $\bar{G}$ & $\mathrm{C}$ & $\overline{\mathrm{A}}$ & $\mathrm{G}$ & $T$ & $\bar{A}$ & $\mathrm{G}$ & $\mathrm{G}$ & $\mathrm{C}$ & $\mathrm{G}$ & $\mathrm{A}$ & $\mathrm{G}$ \\
\hline $\begin{array}{c}\text { AFRIC } \\
\text { A }\end{array}$ & Yoruba & $\begin{array}{c}\text { YRB_JK3 } \\
849\end{array}$ & $\mathrm{~T}$ & $\mathrm{C}$ & $\mathrm{T}$ & $\mathrm{G}$ & $\mathrm{C}$ & $\mathrm{A}$ & $\mathrm{C}$ & $\mathrm{A}$ & $\mathrm{G}$ & $\mathrm{T}$ & $\bar{A}$ & $\mathrm{G}$ & $\mathrm{G}$ & $\mathrm{C}$ & $\mathrm{G}$ & $\mathrm{A}$ & $\mathrm{G}$ \\
\hline $\begin{array}{c}\text { AFRIC } \\
\text { A }\end{array}$ & Yoruba & $\begin{array}{c}\text { YRB_JK3 } \\
849\end{array}$ & $\mathrm{~T}$ & $\mathrm{C}$ & $\mathrm{T}$ & $\mathrm{G}$ & $\mathrm{C}$ & $\bar{G}$ & $\mathrm{C}$ & $\mathrm{A}$ & $\mathrm{G}$ & $\mathrm{T}$ & $\bar{A}$ & $\mathrm{G}$ & $\mathrm{G}$ & $\mathrm{C}$ & $\mathrm{G}$ & $\mathrm{A}$ & $\bar{G}$ \\
\hline $\begin{array}{c}\text { AFRIC } \\
\text { A }\end{array}$ & Yoruba & $\begin{array}{c}\text { YRB_JK3 } \\
850\end{array}$ & $\mathrm{C}$ & $\mathrm{C}$ & $\mathrm{T}$ & $\mathrm{G}$ & $\bar{C}$ & $\mathrm{G}$ & $\bar{C}$ & $\overline{\mathrm{A}}$ & $\mathrm{A}$ & $\mathrm{T}$ & A & $\mathrm{G}$ & $\mathrm{G}$ & $\mathrm{C}$ & $\mathrm{G}$ & $\mathrm{A}$ & $\mathrm{G}$ \\
\hline $\begin{array}{c}\text { AFRIC } \\
\text { A }\end{array}$ & Yoruba & $\begin{array}{c}\text { YRB_JK3 } \\
850\end{array}$ & $\mathrm{~T}$ & $\mathrm{C}$ & $\mathrm{T}$ & $\mathrm{G}$ & $\mathrm{C}$ & $\mathrm{G}$ & $\mathrm{C}$ & $\mathrm{A}$ & $\mathrm{G}$ & $\mathrm{T}$ & $\mathrm{A}$ & $\mathrm{G}$ & G & $\mathrm{C}$ & $\mathrm{G}$ & $\mathrm{A}$ & $\mathrm{G}$ \\
\hline $\begin{array}{c}\text { AFRIC } \\
\text { A }\end{array}$ & Yoruba & $\begin{array}{c}\text { YRB_JK3 } \\
851\end{array}$ & $\mathrm{~T}$ & $\mathrm{C}$ & $\mathrm{T}$ & $\mathrm{G}$ & $\mathrm{C}$ & $\mathrm{G}$ & $\mathrm{C}$ & $\mathrm{A}$ & $\mathrm{G}$ & $\mathrm{T}$ & $\mathrm{A}$ & $\mathrm{G}$ & G & $\mathrm{C}$ & $\mathrm{G}$ & $\mathrm{A}$ & $\mathrm{G}$ \\
\hline $\begin{array}{c}\text { AFRIC } \\
\text { A }\end{array}$ & Yoruba & $\begin{array}{c}\text { YRB_JK3 } \\
851\end{array}$ & $\mathrm{~T}$ & $\mathrm{C}$ & $\mathrm{T}$ & $\mathrm{G}$ & $\mathrm{C}$ & $\mathrm{G}$ & $\mathrm{C}$ & A & $\mathrm{G}$ & $\mathrm{T}$ & $\bar{A}$ & $\mathrm{G}$ & G & $\mathrm{C}$ & $\mathrm{G}$ & $\mathrm{A}$ & $\mathrm{G}$ \\
\hline $\begin{array}{c}\text { AFRIC } \\
\text { A }\end{array}$ & Yoruba & $\begin{array}{c}\text { YRB_JK3 } \\
852\end{array}$ & $\mathrm{~T}$ & $\mathrm{C}$ & $\mathrm{T}$ & $\mathrm{G}$ & $\mathrm{C}$ & $\mathrm{G}$ & $\mathrm{C}$ & $\mathrm{A}$ & $\mathrm{G}$ & $\mathrm{T}$ & $\mathrm{A}$ & $\mathrm{G}$ & G & $\mathrm{C}$ & $\mathrm{G}$ & $\mathrm{A}$ & $\mathrm{G}$ \\
\hline $\begin{array}{c}\text { AFRIC } \\
\text { A }\end{array}$ & Yoruba & $\begin{array}{c}\text { YRB_JK3 } \\
852\end{array}$ & $\mathrm{~T}$ & $\mathrm{C}$ & $\mathrm{T}$ & $\mathrm{G}$ & $\mathrm{C}$ & $\mathrm{G}$ & $\mathrm{C}$ & $\mathrm{A}$ & $\mathrm{G}$ & $\mathrm{T}$ & $\mathrm{A}$ & $\mathrm{G}$ & $\mathrm{G}$ & $\mathrm{C}$ & $\mathrm{G}$ & $\mathrm{A}$ & G \\
\hline
\end{tabular}

John Wiley' 31 Sons, Inc. 


\begin{tabular}{|c|c|c|c|c|c|c|c|c|c|c|c|c|c|c|c|c|c|c|c|}
\hline $\begin{array}{c}\text { AFRIC } \\
\text { A }\end{array}$ & Yoruba & $\begin{array}{c}\text { YRB_JK3 } \\
853\end{array}$ & $\mathrm{~T}$ & $\mathrm{C}$ & $\mathrm{T}$ & $\mathrm{G}$ & $\mathrm{C}$ & $\mathrm{G}$ & $\mathrm{C}$ & $\bar{A}$ & $\mathrm{G}$ & $\mathrm{T}$ & $\mathrm{A}$ & $\bar{G}$ & $\mathrm{G}$ & $\mathrm{C}$ & $\mathrm{G}$ & $\mathrm{A}$ & $\bar{G}$ \\
\hline $\begin{array}{c}\text { AFRIC } \\
\text { A }\end{array}$ & Yoruba & $\begin{array}{c}\text { YRB_JK3 } \\
853\end{array}$ & $\mathrm{~T}$ & C & $\mathrm{T}$ & G & $\mathrm{C}$ & G & $\mathrm{C}$ & $\mathrm{A}$ & G & $\mathrm{T}$ & $\mathrm{A}$ & G & $\mathrm{A}$ & $\mathrm{C}$ & G & $\mathrm{A}$ & $\mathrm{G}$ \\
\hline $\begin{array}{c}\text { AFRIC } \\
\text { A }\end{array}$ & Yoruba & $\begin{array}{c}\text { YRB_JK3 } \\
854\end{array}$ & $\mathrm{C}$ & $\mathrm{C}$ & $\mathrm{T}$ & $\mathrm{G}$ & $\bar{C}$ & $\mathrm{G}$ & $\mathrm{C}$ & $\mathrm{A}$ & $\mathrm{G}$ & $\mathrm{T}$ & $\mathrm{A}$ & $\mathrm{A}$ & $\mathrm{G}$ & $\mathrm{C}$ & $\mathrm{G}$ & $\mathrm{A}$ & $\mathrm{G}$ \\
\hline $\begin{array}{c}\text { AFRIC } \\
\text { A }\end{array}$ & Yoruba & $\begin{array}{c}\text { YRB_JK3 } \\
854 \\
\end{array}$ & $\mathrm{~T}$ & $\mathrm{C}$ & $\mathrm{T}$ & $\mathrm{G}$ & $\mathrm{C}$ & $\mathrm{G}$ & $\mathrm{C}$ & $\mathrm{A}$ & $\mathrm{G}$ & $\mathrm{T}$ & $\mathrm{A}$ & $\mathrm{A}$ & $\mathrm{G}$ & C & G & $\mathrm{A}$ & $\mathrm{G}$ \\
\hline $\begin{array}{c}\text { AFRIC } \\
\text { A }\end{array}$ & Yoruba & $\begin{array}{c}\text { YRB_JK3 } \\
855\end{array}$ & $\mathrm{~T}$ & $\mathrm{C}$ & $\mathrm{T}$ & $\mathrm{G}$ & $\mathrm{C}$ & $\mathrm{G}$ & $\mathrm{C}$ & $\mathrm{A}$ & $\mathrm{G}$ & $\mathrm{T}$ & $\mathrm{A}$ & $\mathrm{G}$ & $\mathrm{G}$ & $\mathrm{C}$ & $\mathrm{G}$ & $\mathrm{A}$ & $\mathrm{G}$ \\
\hline $\begin{array}{c}\text { AFRIC } \\
\text { A }\end{array}$ & Yoruba & $\begin{array}{c}\text { YRB_JK3 } \\
855\end{array}$ & $\mathrm{~T}$ & $\mathrm{C}$ & $\mathrm{T}$ & $\mathrm{G}$ & $\mathrm{C}$ & $\mathrm{G}$ & $\mathrm{C}$ & A & $\mathrm{G}$ & $\mathrm{T}$ & $\mathrm{A}$ & $\mathrm{G}$ & $\mathrm{G}$ & $\mathrm{C}$ & $\mathrm{G}$ & A & $\mathrm{G}$ \\
\hline $\begin{array}{c}\text { AFRIC } \\
\text { A }\end{array}$ & Yoruba & $\begin{array}{c}\text { YRB_JK3 } \\
856\end{array}$ & $\mathrm{C}$ & $\mathrm{C}$ & $\mathrm{T}$ & $\mathrm{G}$ & $\mathrm{C}$ & $\mathrm{G}$ & $\mathrm{C}$ & $\mathrm{A}$ & $\mathrm{G}$ & $\mathrm{T}$ & $\mathrm{A}$ & $\mathrm{A}$ & $\mathrm{G}$ & $\mathrm{C}$ & $\mathrm{G}$ & $\mathrm{A}$ & $\mathrm{G}$ \\
\hline $\begin{array}{c}\text { AFRIC } \\
\text { A }\end{array}$ & Yoruba & $\begin{array}{c}\text { YRB_JK3 } \\
856\end{array}$ & $\mathrm{~T}$ & $\mathrm{C}$ & $\mathrm{T}$ & $\mathrm{G}$ & $\mathrm{C}$ & G & $\mathrm{C}$ & $\mathrm{A}$ & $\mathrm{G}$ & $\mathrm{T}$ & $\mathrm{A}$ & G & $\mathrm{G}$ & $\mathrm{C}$ & G & $\mathrm{A}$ & G \\
\hline $\begin{array}{c}\text { AFRIC } \\
\text { A }\end{array}$ & Yoruba & $\begin{array}{c}\text { YRB_JK3 } \\
860\end{array}$ & $\mathrm{~T}$ & $\mathrm{C}$ & $\mathrm{T}$ & $\mathrm{G}$ & $\mathrm{C}$ & $\mathrm{G}$ & $\mathrm{C}$ & A & $\mathrm{G}$ & $\mathrm{T}$ & $\mathrm{A}$ & $\mathrm{G}$ & $\mathrm{G}$ & $\mathrm{C}$ & $\mathrm{G}$ & A & G \\
\hline $\begin{array}{c}\text { AFRIC } \\
\text { A }\end{array}$ & Yoruba & $\begin{array}{c}\text { YRB_JK3 } \\
860\end{array}$ & $\mathrm{C}$ & $\mathrm{C}$ & $\mathrm{T}$ & $\mathrm{G}$ & $\mathrm{C}$ & $G$ & $\mathrm{C}$ & $\mathrm{A}$ & $\mathrm{G}$ & $\mathrm{T}$ & $\mathrm{A}$ & G & $\mathrm{G}$ & C & $\mathrm{G}$ & A & $\bar{G}$ \\
\hline $\begin{array}{c}\text { AFRIC } \\
\text { A }\end{array}$ & Yoruba & $\begin{array}{c}\text { YRB_JK3 } \\
858 \\
\end{array}$ & $\mathrm{~T}$ & $\mathrm{C}$ & $\mathrm{T}$ & $\mathrm{G}$ & $\mathrm{C}$ & $\mathrm{G}$ & $\mathrm{C}$ & $\mathrm{A}$ & $\mathrm{G}$ & $\mathrm{T}$ & $\mathrm{A}$ & $\mathrm{G}$ & $\mathrm{G}$ & $\mathrm{C}$ & $\mathrm{G}$ & $\mathrm{A}$ & $\bar{G}$ \\
\hline $\begin{array}{c}\text { AFRIC } \\
\text { A }\end{array}$ & Yoruba & $\begin{array}{c}\text { YRB_JK3 } \\
858\end{array}$ & $\mathrm{C}$ & $\mathrm{C}$ & $\mathrm{T}$ & $\mathrm{G}$ & $\mathrm{C}$ & $\mathrm{G}$ & $\mathrm{C}$ & $\mathrm{A}$ & $\mathrm{G}$ & $\mathrm{T}$ & $\mathrm{A}$ & $\mathrm{G}$ & $\mathrm{G}$ & $\mathrm{C}$ & $\mathrm{G}$ & A & $\mathrm{G}$ \\
\hline $\begin{array}{c}\text { AFRIC } \\
\text { A }\end{array}$ & Yoruba & $\begin{array}{c}\text { YRB_JK3 } \\
859\end{array}$ & $\mathrm{~T}$ & $\mathrm{C}$ & $\mathrm{T}$ & $\mathrm{G}$ & $\mathrm{C}$ & $\mathrm{G}$ & $\mathrm{C}$ & $\mathrm{A}$ & $\mathrm{G}$ & $\mathrm{T}$ & $\mathrm{A}$ & $\mathrm{G}$ & $\mathrm{G}$ & $\mathrm{C}$ & $\mathrm{G}$ & $\mathrm{A}$ & $\bar{G}$ \\
\hline $\begin{array}{c}\text { AFRIC } \\
\text { A }\end{array}$ & Yoruba & $\begin{array}{c}\text { YRB_JK3 } \\
859\end{array}$ & $\mathrm{~T}$ & $\mathrm{C}$ & $\mathrm{T}$ & $\mathrm{G}$ & $\mathrm{C}$ & $\mathrm{G}$ & $\mathrm{C}$ & $\mathrm{A}$ & $\mathrm{G}$ & $\mathrm{T}$ & $\mathrm{A}$ & $\bar{G}$ & $\mathrm{G}$ & $\mathrm{C}$ & $\mathrm{G}$ & $\bar{A}$ & $\overline{\mathrm{G}}$ \\
\hline $\begin{array}{l}\text { East- } \\
\text { Asia }\end{array}$ & China & $\underset{5}{\mathrm{CH}} \mathrm{CH} 2$ & $\mathrm{~T}$ & $\mathrm{C}$ & $\mathrm{T}$ & $\mathrm{G}$ & $\mathrm{C}$ & $\mathrm{G}$ & $\mathrm{C}$ & $\mathrm{A}$ & $\mathrm{G}$ & $\mathrm{T}$ & $\mathrm{A}$ & $\mathrm{G}$ & $\mathrm{G}$ & $\bar{C}$ & $\mathrm{G}$ & $\mathrm{A}$ & $\bar{G}$ \\
\hline $\begin{array}{c}\text { East- } \\
\text { Asia } \\
\end{array}$ & China & $\underset{5}{\mathrm{CH}}$ & $\mathrm{T}$ & $\mathrm{C}$ & $\mathrm{T}$ & $\mathrm{G}$ & $\mathrm{C}$ & $\mathrm{G}$ & $\mathrm{C}$ & A & $\mathrm{G}$ & $\mathrm{T}$ & $\mathrm{A}$ & $\mathrm{G}$ & $\mathrm{G}$ & $\mathrm{C}$ & $\mathrm{G}$ & $\mathrm{A}$ & $\mathrm{G}$ \\
\hline $\begin{array}{l}\text { East- } \\
\text { Asia }\end{array}$ & China & $\underset{2}{\mathrm{CH}+\mathrm{CHO}}$ & $\mathrm{T}$ & $\mathrm{C}$ & $\mathrm{T}$ & $\mathrm{G}$ & $\mathrm{C}$ & $\mathrm{G}$ & $\mathrm{C}$ & $\mathrm{A}$ & $\mathrm{G}$ & $\mathrm{T}$ & $\mathrm{A}$ & $\mathrm{G}$ & $\mathrm{G}$ & $\mathrm{C}$ & $\mathrm{G}$ & $\mathrm{A}$ & $\mathrm{G}$ \\
\hline $\begin{array}{l}\text { East- } \\
\text { Asia }\end{array}$ & China & $\underset{2}{\mathrm{CH} \_\mathrm{CHO}}$ & $\mathrm{T}$ & $\mathrm{C}$ & $\mathrm{T}$ & $\mathrm{G}$ & $\mathrm{C}$ & $\mathrm{G}$ & $\mathrm{C}$ & $\mathrm{A}$ & $\mathrm{G}$ & $\mathrm{T}$ & $\mathrm{A}$ & $\mathrm{G}$ & $\mathrm{G}$ & $\mathrm{C}$ & G & A & G \\
\hline $\begin{array}{c}\text { East- } \\
\text { Asia }\end{array}$ & China & $\underset{3}{\mathrm{CH}} \mathrm{CHO}$ & $\mathrm{T}$ & $\mathrm{C}$ & $\mathrm{T}$ & $\mathrm{G}$ & $\mathrm{C}$ & $\mathrm{G}$ & $\mathrm{C}$ & $\mathrm{A}$ & $\mathrm{G}$ & $\mathrm{T}$ & $\mathrm{A}$ & $\mathrm{G}$ & $\mathrm{G}$ & $\mathrm{C}$ & G & $\mathrm{A}$ & $\mathrm{G}$ \\
\hline $\begin{array}{l}\text { East- } \\
\text { Asia }\end{array}$ & China & $\underset{3}{\mathrm{CH}}$ & $\mathrm{T}$ & $\mathrm{C}$ & $\mathrm{T}$ & $\mathrm{G}$ & $\mathrm{C}$ & $\mathrm{G}$ & $\mathrm{C}$ & A & G & $\mathrm{T}$ & $\mathrm{A}$ & $\mathrm{G}$ & $\mathrm{G}$ & $\mathrm{C}$ & G & $\mathrm{A}$ & G \\
\hline $\begin{array}{c}\text { East- } \\
\text { Asia }\end{array}$ & China & $\underset{4}{\mathrm{CH} \_\mathrm{CHO}}$ & $\mathrm{T}$ & $\mathrm{C}$ & $\mathrm{T}$ & $\mathrm{G}$ & $\mathrm{C}$ & $\mathrm{G}$ & $\mathrm{C}$ & $\mathrm{A}$ & $\mathrm{G}$ & $\mathrm{T}$ & $\mathrm{A}$ & $\mathrm{G}$ & $\mathrm{G}$ & $\mathrm{C}$ & G & A & G \\
\hline $\begin{array}{l}\text { East- } \\
\text { Asia }\end{array}$ & China & $\underset{4}{\mathrm{CH}} \mathrm{CHO}$ & $\mathrm{T}$ & $\mathrm{C}$ & $\mathrm{T}$ & $\mathrm{G}$ & $\mathrm{C}$ & $\mathrm{G}$ & $\mathrm{C}$ & $\mathrm{A}$ & $\mathrm{G}$ & $\mathrm{T}$ & $\mathrm{A}$ & $\mathrm{G}$ & $\mathrm{G}$ & $\mathrm{C}$ & $\mathrm{T}$ & $\mathrm{A}$ & G \\
\hline $\begin{array}{l}\text { East- } \\
\text { Asia }\end{array}$ & China & $\underset{5}{\mathrm{CH}}$ & $\mathrm{T}$ & $\mathrm{C}$ & $\mathrm{T}$ & $\mathrm{G}$ & $\mathrm{C}$ & $\mathrm{G}$ & $\mathrm{C}$ & A & G & $\mathrm{T}$ & $\mathrm{A}$ & $\mathrm{G}$ & $\mathrm{G}$ & $\mathrm{C}$ & G & $\mathrm{A}$ & $\mathrm{G}$ \\
\hline
\end{tabular}

John Wiley 32 \& Sons, Inc. 


\begin{tabular}{|c|c|c|c|c|c|c|c|c|c|c|c|c|c|c|c|c|c|c|c|}
\hline $\begin{array}{l}\text { East- } \\
\text { Asia } \\
\end{array}$ & China & $\frac{\mathrm{CH}_{5} \mathrm{CHO}}{\mathrm{C}}$ & $\mathrm{T}$ & $\mathrm{C}$ & $\mathrm{T}$ & $\bar{A}$ & C & $\mathrm{G}$ & $\mathrm{C}$ & $\mathrm{A}$ & $\bar{G}$ & $\mathrm{~T}$ & $\mathrm{~A}$ & $\bar{G}$ & $\mathrm{G}$ & $\mathrm{C}$ & $\mathrm{G}$ & $\mathrm{A}$ & $\bar{G}$ \\
\hline $\begin{array}{l}\text { East- } \\
\text { Asia } \\
\end{array}$ & China & $\underset{6}{\mathrm{CH}+\mathrm{CHO}}$ & $\mathrm{T}$ & C & $\mathrm{T}$ & G & $\mathrm{C}$ & $\mathrm{G}$ & $\mathrm{C}$ & A & $\mathrm{G}$ & $\mathrm{T}$ & $\mathrm{A}$ & G & G & $\mathrm{C}$ & $\mathrm{G}$ & $\mathrm{A}$ & $\mathrm{G}$ \\
\hline $\begin{array}{l}\text { East- } \\
\text { Asia } \\
\end{array}$ & China & $\begin{array}{c}\mathrm{CH}+\mathrm{CHO} \\
6\end{array}$ & $\mathrm{~T}$ & $\mathrm{C}$ & $\mathrm{T}$ & $\bar{G}$ & $\mathrm{C}$ & $\mathrm{G}$ & $\mathrm{C}$ & $\mathrm{A}$ & $\mathrm{G}$ & $\mathrm{T}$ & $\mathrm{A}$ & $\mathrm{G}$ & $\mathrm{G}$ & $\mathrm{C}$ & $\mathrm{G}$ & $\mathrm{A}$ & $\mathrm{G}$ \\
\hline $\begin{array}{l}\text { East- } \\
\text { Asia } \\
\end{array}$ & China & $\underset{7}{\mathrm{CH}+\mathrm{CHO}}$ & $\mathrm{T}$ & C & $\mathrm{T}$ & G & $\mathrm{C}$ & $\mathrm{G}$ & $\mathrm{C}$ & A & $\mathrm{G}$ & $\mathrm{T}$ & $\mathrm{A}$ & G & $\mathrm{G}$ & $\mathrm{C}$ & $\mathrm{G}$ & $\mathrm{A}$ & $\mathrm{G}$ \\
\hline $\begin{array}{l}\text { East- } \\
\text { Asia } \\
\end{array}$ & China & $\frac{\mathrm{CH}}{7}$ & $\mathrm{~T}$ & $\mathrm{C}$ & $\mathrm{T}$ & $\mathrm{G}$ & $\mathrm{C}$ & $\mathrm{G}$ & $\mathrm{C}$ & A & $\mathrm{G}$ & $\mathrm{T}$ & $\mathrm{A}$ & $\mathrm{G}$ & $\mathrm{G}$ & $\mathrm{C}$ & $\mathrm{G}$ & $\mathrm{A}$ & $\bar{G}$ \\
\hline $\begin{array}{l}\text { East- } \\
\text { Asia } \\
\end{array}$ & China & $\begin{array}{c}\mathrm{CH}+\mathrm{CHO} \\
\end{array}$ & $\mathrm{T}$ & $\mathrm{C}$ & $\mathrm{T}$ & G & $\mathrm{C}$ & $\mathrm{G}$ & $\mathrm{C}$ & $\mathrm{A}$ & $\mathrm{G}$ & $\mathrm{T}$ & $\mathrm{A}$ & $\mathrm{G}$ & $\mathrm{G}$ & $\mathrm{C}$ & $\mathrm{G}$ & A & $\mathrm{G}$ \\
\hline $\begin{array}{l}\text { East- } \\
\text { Asia }\end{array}$ & China & $\begin{array}{c}\mathrm{CH}+\mathrm{CHO} \\
8\end{array}$ & $\mathrm{~T}$ & $\mathrm{C}$ & $\mathrm{T}$ & $\mathrm{G}$ & $\mathrm{C}$ & $\mathrm{G}$ & $\mathrm{C}$ & $\mathrm{A}$ & $\mathrm{G}$ & $\mathrm{T}$ & $\mathrm{A}$ & $\mathrm{G}$ & $\mathrm{G}$ & $\mathrm{C}$ & $\mathrm{G}$ & $\mathrm{A}$ & $\bar{G}$ \\
\hline $\begin{array}{l}\text { East- } \\
\text { Asia }\end{array}$ & China & $\underset{9}{\mathrm{CH}+\mathrm{CHO}}$ & $\mathrm{T}$ & $\mathrm{C}$ & $\mathrm{T}$ & $\mathrm{G}$ & $\mathrm{C}$ & $\mathrm{G}$ & $\mathrm{C}$ & $\mathrm{A}$ & $\mathrm{G}$ & $\mathrm{T}$ & $\mathrm{A}$ & G & $\mathrm{G}$ & $\mathrm{C}$ & $\mathrm{G}$ & $\mathrm{A}$ & $\mathrm{G}$ \\
\hline $\begin{array}{l}\text { East- } \\
\text { Asia } \\
\end{array}$ & China & $\underset{9}{\mathrm{CH}}$ & $\mathrm{T}$ & $\mathrm{C}$ & $\mathrm{T}$ & G & $\mathrm{C}$ & $\mathrm{G}$ & $\mathrm{C}$ & $\mathrm{A}$ & $\mathrm{G}$ & $\mathrm{T}$ & $\mathrm{A}$ & $\mathrm{G}$ & $\mathrm{G}$ & $\mathrm{C}$ & $\mathrm{G}$ & $\mathrm{A}$ & $\mathrm{G}$ \\
\hline $\begin{array}{l}\text { East- } \\
\text { Asia } \\
\end{array}$ & China & $\begin{array}{c}\mathrm{CH}+\mathrm{CH} 1 \\
0\end{array}$ & $\mathrm{~T}$ & $\mathrm{C}$ & $\mathrm{T}$ & G & $\mathrm{C}$ & $\mathrm{G}$ & $\mathrm{C}$ & $\mathrm{A}$ & $\mathrm{G}$ & $\mathrm{T}$ & $\mathrm{A}$ & G & G & $\mathrm{C}$ & $\mathrm{G}$ & $\mathrm{A}$ & $\bar{G}$ \\
\hline $\begin{array}{l}\text { East- } \\
\text { Asia } \\
\end{array}$ & China & $\begin{array}{c}\mathrm{CH} \text { CH1 } \\
0 \\
\end{array}$ & $\mathrm{~T}$ & $\mathrm{C}$ & $\mathrm{T}$ & $\mathrm{G}$ & $\mathrm{C}$ & $\mathrm{G}$ & $\mathrm{C}$ & $\mathrm{A}$ & $\mathrm{G}$ & $\mathrm{T}$ & $\mathrm{A}$ & $\mathrm{G}$ & $\mathrm{G}$ & $\mathrm{C}$ & $\mathrm{G}$ & $\mathrm{A}$ & $\bar{G}$ \\
\hline $\begin{array}{l}\text { East- } \\
\text { Asia } \\
\end{array}$ & China & $\underset{-1}{\mathrm{CH}} \mathrm{CH} 1$ & $\mathrm{~T}$ & $\mathrm{C}$ & $\mathrm{T}$ & G & $\mathrm{C}$ & $\mathrm{G}$ & $\mathrm{C}$ & $\mathrm{A}$ & $\mathrm{G}$ & $\mathrm{T}$ & $\mathrm{A}$ & $\mathrm{G}$ & $\mathrm{G}$ & $\mathrm{C}$ & $\mathrm{G}$ & $\mathrm{A}$ & $\mathrm{G}$ \\
\hline $\begin{array}{l}\text { East- } \\
\text { Asia } \\
\end{array}$ & China & $\underset{1}{\mathrm{CH}}$ & $\mathrm{T}$ & $\mathrm{C}$ & $\mathrm{T}$ & $\mathrm{G}$ & $\mathrm{C}$ & $\mathrm{G}$ & $\mathrm{C}$ & $\mathrm{A}$ & $\mathrm{G}$ & $\mathrm{T}$ & $\mathrm{A}$ & $\mathrm{G}$ & $\bar{G}$ & $\mathrm{C}$ & $\mathrm{G}$ & $\mathrm{A}$ & $\bar{G}$ \\
\hline $\begin{array}{l}\text { East- } \\
\text { Asia } \\
\end{array}$ & China & $\begin{array}{c}\mathrm{CH}+\mathrm{CH} 1 \\
2\end{array}$ & $\mathrm{~T}$ & $\mathrm{C}$ & $\mathrm{T}$ & G & $\mathrm{C}$ & $\mathrm{G}$ & $\mathrm{C}$ & $\mathrm{A}$ & $\mathrm{G}$ & $\mathrm{T}$ & $\mathrm{A}$ & $\mathrm{G}$ & $\mathrm{G}$ & $\mathrm{C}$ & $\mathrm{G}$ & $\mathrm{A}$ & $\bar{G}$ \\
\hline $\begin{array}{l}\text { East- } \\
\text { Asia } \\
\end{array}$ & China & $\underset{2}{\mathrm{CH}+\mathrm{CH} 1}$ & $\mathrm{~T}$ & $\mathrm{C}$ & $\mathrm{T}$ & $\bar{G}$ & $\mathrm{C}$ & $\mathrm{G}$ & $\mathrm{C}$ & $\mathrm{A}$ & $\mathrm{G}$ & $\mathrm{T}$ & $\bar{A}$ & $\mathrm{G}$ & $\mathrm{G}$ & $\mathrm{C}$ & $\mathrm{G}$ & $\mathrm{A}$ & $\bar{G}$ \\
\hline $\begin{array}{l}\text { East- } \\
\text { Asia } \\
\end{array}$ & China & $\frac{\mathrm{CH}_{3} \mathrm{CH} 1}{\mathrm{C}}$ & $\mathrm{T}$ & $\mathrm{C}$ & $\mathrm{T}$ & $\bar{G}$ & $\mathrm{C}$ & $\mathrm{G}$ & $\mathrm{C}$ & $\mathrm{A}$ & $\mathrm{G}$ & $\mathrm{T}$ & $\mathrm{A}$ & $\mathrm{G}$ & $\mathrm{G}$ & $\mathrm{C}$ & $\mathrm{G}$ & $\mathrm{A}$ & $\bar{G}$ \\
\hline $\begin{array}{l}\text { East- } \\
\text { Asia } \\
\end{array}$ & China & $\frac{\mathrm{CH}_{3} \mathrm{CH} 1}{\mathrm{C}}$ & $\mathrm{T}$ & $\mathrm{C}$ & $\mathrm{T}$ & $\bar{G}$ & $\mathrm{C}$ & $\mathrm{G}$ & $\mathrm{C}$ & $\mathrm{A}$ & $\mathrm{G}$ & $\mathrm{T}$ & $\bar{A}$ & G & $\mathrm{G}$ & $\mathrm{C}$ & $\mathrm{G}$ & $\mathrm{A}$ & $\bar{G}$ \\
\hline $\begin{array}{l}\text { East- } \\
\text { Asia } \\
\end{array}$ & China & $\underset{4}{\mathrm{CH}}$ & $\mathrm{T}$ & $\mathrm{C}$ & $\mathrm{T}$ & G & $\mathrm{C}$ & $\mathrm{G}$ & $\mathrm{C}$ & $\mathrm{A}$ & $\mathrm{G}$ & $\mathrm{T}$ & $\mathrm{A}$ & G & $\mathrm{G}$ & $\mathrm{C}$ & $\mathrm{G}$ & $\mathrm{A}$ & $\bar{G}$ \\
\hline $\begin{array}{l}\text { East- } \\
\text { Asia } \\
\end{array}$ & China & $\underset{4}{\mathrm{CH}} \mathrm{CH} 1$ & $\mathrm{~T}$ & $\mathrm{C}$ & $\mathrm{T}$ & G & $\mathrm{C}$ & $\mathrm{G}$ & $\mathrm{C}$ & $\mathrm{A}$ & $\mathrm{G}$ & $\mathrm{T}$ & $\mathrm{A}$ & $\mathrm{G}$ & $\mathrm{G}$ & $\mathrm{C}$ & $\mathrm{G}$ & $\mathrm{A}$ & $\mathrm{G}$ \\
\hline $\begin{array}{l}\text { East- } \\
\text { Asia } \\
\end{array}$ & China & $\frac{\mathrm{CH}+\mathrm{CH} 1}{5}$ & $\mathrm{~T}$ & $\mathrm{C}$ & $\mathrm{T}$ & G & $\bar{C}$ & $\mathrm{G}$ & $\mathrm{C}$ & $\mathrm{A}$ & $\mathrm{G}$ & $\mathrm{T}$ & $\mathrm{A}$ & G & $\mathrm{G}$ & $\mathrm{C}$ & G & A & $\mathrm{G}$ \\
\hline $\begin{array}{l}\text { East- } \\
\text { Asia } \\
\end{array}$ & China & $\underset{5}{\mathrm{CH}}$ & $\mathrm{T}$ & $\mathrm{C}$ & $\mathrm{T}$ & G & $\mathrm{C}$ & $\mathrm{G}$ & $\mathrm{C}$ & $\mathrm{A}$ & $\mathrm{G}$ & $\mathrm{T}$ & $\mathrm{A}$ & $\mathrm{G}$ & $\mathrm{G}$ & $\mathrm{C}$ & $\mathrm{G}$ & $\mathrm{A}$ & $\mathrm{G}$ \\
\hline $\begin{array}{l}\text { East- } \\
\text { Asia } \\
\end{array}$ & China & $\underset{6}{\mathrm{CH}} \mathrm{CH} 1$ & $\mathrm{~T}$ & $\mathrm{C}$ & $\mathrm{T}$ & G & $\mathrm{C}$ & $\mathrm{G}$ & $\mathrm{C}$ & $\mathrm{A}$ & $\mathrm{G}$ & $\mathrm{T}$ & $\mathrm{A}$ & $\mathrm{G}$ & $\mathrm{G}$ & $\mathrm{C}$ & $\mathrm{G}$ & $\mathrm{A}$ & $\mathrm{G}$ \\
\hline $\begin{array}{l}\text { East- } \\
\text { Asia } \\
\end{array}$ & China & $\begin{array}{c}\mathrm{CH}+\mathrm{CH} 1 \\
\end{array}$ & $\mathrm{~T}$ & $\mathrm{C}$ & $\mathrm{T}$ & G & $\bar{C}$ & $\mathrm{G}$ & $\mathrm{C}$ & $\mathrm{A}$ & $\mathrm{G}$ & $\mathrm{T}$ & $\mathrm{A}$ & G & $\mathrm{G}$ & $\mathrm{C}$ & $\mathrm{T}$ & $\mathrm{A}$ & $\mathrm{G}$ \\
\hline
\end{tabular}

John Wiley 33 Sons, Inc. 


\begin{tabular}{|c|c|c|c|c|c|c|c|c|c|c|c|c|c|c|c|c|c|c|c|}
\hline $\begin{array}{l}\text { East- } \\
\text { Asia }\end{array}$ & China & $\mathrm{CH}_{7}^{\mathrm{CH} 1}$ & $\mathrm{~T}$ & $\mathrm{C}$ & $\mathrm{T}$ & $\mathrm{G}$ & $\mathrm{C}$ & $\mathrm{G}$ & $\mathrm{C}$ & $\mathrm{A}$ & $\mathrm{G}$ & $\mathrm{T}$ & $\mathrm{A}$ & $\mathrm{G}$ & $\mathrm{G}$ & $\mathrm{C}$ & $\mathrm{G}$ & $\mathrm{A}$ & $\mathrm{G}$ \\
\hline $\begin{array}{l}\text { East- } \\
\text { Asia } \\
\end{array}$ & China & $\underset{7}{\mathrm{CH}+\mathrm{CH} 1}$ & $\mathrm{~T}$ & C & $\mathrm{T}$ & G & $\mathrm{C}$ & G & $\mathrm{C}$ & $\mathrm{A}$ & $\mathrm{G}$ & $\mathrm{T}$ & $\mathrm{A}$ & G & $\mathrm{G}$ & $\mathrm{C}$ & $\mathrm{G}$ & $\mathrm{A}$ & $\mathrm{G}$ \\
\hline $\begin{array}{l}\text { East- } \\
\text { Asia } \\
\end{array}$ & China & $\begin{array}{c}\mathrm{CH} \_\mathrm{CH} 1 \\
8\end{array}$ & $\mathrm{~T}$ & $\mathrm{C}$ & $\mathrm{T}$ & $\mathrm{G}$ & $\mathrm{C}$ & $\mathrm{G}$ & $\mathrm{C}$ & $\bar{A}$ & $\mathrm{G}$ & $\mathrm{T}$ & $\mathrm{A}$ & $\mathrm{G}$ & $\mathrm{G}$ & $\mathrm{C}$ & $\mathrm{G}$ & $\overline{\mathrm{A}}$ & $\mathrm{G}$ \\
\hline $\begin{array}{l}\text { East- } \\
\text { Asia } \\
\end{array}$ & China & $\begin{array}{c}\mathrm{CH}+\mathrm{CH} 1 \\
8\end{array}$ & $\mathrm{~T}$ & $\mathrm{C}$ & $\mathrm{T}$ & $\mathrm{G}$ & $\mathrm{G}$ & $\mathrm{G}$ & $\mathrm{C}$ & $\mathrm{A}$ & $\mathrm{G}$ & $\mathrm{T}$ & $\mathrm{A}$ & G & $\mathrm{G}$ & C & $\mathrm{G}$ & A & $\mathrm{G}$ \\
\hline $\begin{array}{l}\text { East- } \\
\text { Asia } \\
\end{array}$ & China & $\begin{array}{c}\mathrm{CH}+\mathrm{CH} 1 \\
9\end{array}$ & $\mathrm{~T}$ & $\mathrm{C}$ & $\mathrm{T}$ & $\mathrm{G}$ & $\mathrm{C}$ & $\mathrm{G}$ & $\mathrm{C}$ & $\mathrm{A}$ & $\mathrm{G}$ & $\mathrm{T}$ & $\mathrm{A}$ & $\mathrm{G}$ & $\mathrm{G}$ & $\mathrm{C}$ & $\mathrm{G}$ & $\mathrm{A}$ & $\mathrm{G}$ \\
\hline $\begin{array}{l}\text { East- } \\
\text { Asia } \\
\end{array}$ & China & $\underset{9}{\mathrm{CH}} \mathrm{CH} 1$ & $\mathrm{~T}$ & $\mathrm{C}$ & $\mathrm{T}$ & $\mathrm{G}$ & $\mathrm{C}$ & $\mathrm{G}$ & $\mathrm{C}$ & $\mathrm{A}$ & $\mathrm{G}$ & $\mathrm{T}$ & $\mathrm{A}$ & $\mathrm{G}$ & $\mathrm{G}$ & $\mathrm{C}$ & $\mathrm{G}$ & A & $\mathrm{G}$ \\
\hline $\begin{array}{l}\text { East- } \\
\text { Asia } \\
\end{array}$ & China & $\begin{array}{c}\mathrm{CH} \text { _CH2 } \\
0\end{array}$ & $\mathrm{~T}$ & $\mathrm{C}$ & $\mathrm{T}$ & $\mathrm{G}$ & $\mathrm{C}$ & $\mathrm{G}$ & $\mathrm{C}$ & $\mathrm{A}$ & $\mathrm{G}$ & $\mathrm{T}$ & $\mathrm{A}$ & $\mathrm{G}$ & $\mathrm{G}$ & $\mathrm{C}$ & $\mathrm{G}$ & $\mathrm{A}$ & $\mathrm{G}$ \\
\hline $\begin{array}{l}\text { East- } \\
\text { Asia } \\
\end{array}$ & China & $\begin{array}{c}\mathrm{CH}+\mathrm{CH} 2 \\
0 \\
\end{array}$ & $\mathrm{~T}$ & $\mathrm{C}$ & $\mathrm{T}$ & $\mathrm{G}$ & $\mathrm{C}$ & G & $\mathrm{C}$ & $\mathrm{A}$ & $\mathrm{G}$ & $\mathrm{T}$ & $\mathrm{A}$ & G & $\mathrm{G}$ & C & $\mathrm{G}$ & $\mathrm{A}$ & $\mathrm{G}$ \\
\hline $\begin{array}{l}\text { East- } \\
\text { Asia } \\
\end{array}$ & China & $\underset{1}{\mathrm{CH}} \mathrm{CH} 2$ & $\mathrm{~T}$ & $\mathrm{C}$ & $\mathrm{T}$ & $\mathrm{G}$ & $\mathrm{C}$ & $\mathrm{G}$ & $\mathrm{C}$ & $\mathrm{A}$ & $\mathrm{G}$ & $\mathrm{T}$ & $\mathrm{A}$ & $\mathrm{G}$ & $\mathrm{G}$ & $\mathrm{C}$ & $\mathrm{G}$ & $\mathrm{A}$ & $\mathrm{G}$ \\
\hline $\begin{array}{l}\text { East- } \\
\text { Asia } \\
\end{array}$ & China & $\underset{1}{\mathrm{CH}+\mathrm{CH} 2}$ & $\mathrm{~T}$ & $\mathrm{C}$ & $\mathrm{T}$ & $\mathrm{G}$ & $\mathrm{C}$ & $G$ & $\mathrm{C}$ & $\mathrm{A}$ & $\mathrm{G}$ & $\mathrm{T}$ & $\mathrm{A}$ & G & $\mathrm{G}$ & $\mathrm{C}$ & $\mathrm{G}$ & $\mathrm{A}$ & $\mathrm{G}$ \\
\hline $\begin{array}{l}\text { East- } \\
\text { Asia } \\
\end{array}$ & China & $\underset{2}{\mathrm{CH}}$ & $\mathrm{T}$ & $\mathrm{C}$ & $\mathrm{T}$ & $\mathrm{G}$ & $\mathrm{C}$ & $\mathrm{G}$ & $\mathrm{C}$ & $\mathrm{A}$ & $\mathrm{G}$ & $\mathrm{T}$ & $\mathrm{A}$ & $\mathrm{G}$ & $\mathrm{G}$ & $\mathrm{C}$ & $\mathrm{G}$ & $\mathrm{A}$ & $\mathrm{G}$ \\
\hline $\begin{array}{l}\text { East- } \\
\text { Asia }\end{array}$ & China & $\underset{2}{\mathrm{CH}+\mathrm{CH} 2}$ & $\mathrm{~T}$ & $\mathrm{C}$ & $\mathrm{T}$ & $\mathrm{G}$ & $\mathrm{C}$ & $\mathrm{G}$ & $\mathrm{C}$ & $\mathrm{A}$ & $\mathrm{G}$ & $\mathrm{T}$ & $\mathrm{A}$ & $\mathrm{G}$ & $\mathrm{G}$ & $\mathrm{C}$ & $\mathrm{G}$ & $\mathrm{A}$ & $\mathrm{G}$ \\
\hline $\begin{array}{l}\text { East- } \\
\text { Asia } \\
\end{array}$ & China & $\underset{3}{\mathrm{CH}+\mathrm{CH} 2}$ & $\mathrm{~T}$ & $\mathrm{C}$ & $\mathrm{T}$ & $\mathrm{G}$ & $\mathrm{C}$ & $\mathrm{G}$ & $\mathrm{C}$ & $\mathrm{A}$ & $\mathrm{G}$ & $\mathrm{T}$ & $\mathrm{A}$ & $\mathrm{G}$ & $\mathrm{G}$ & $\mathrm{C}$ & $\mathrm{G}$ & $\mathrm{A}$ & $\mathrm{G}$ \\
\hline $\begin{array}{l}\text { East- } \\
\text { Asia } \\
\end{array}$ & China & $\begin{array}{c}\mathrm{CH}-\mathrm{CH} 2 \\
3 \\
\end{array}$ & $\mathrm{~T}$ & $\mathrm{C}$ & $\mathrm{T}$ & $\mathrm{G}$ & $\mathrm{C}$ & $\mathrm{G}$ & $\mathrm{C}$ & $\mathrm{A}$ & $\mathrm{G}$ & $\mathrm{T}$ & $\mathrm{A}$ & $\mathrm{G}$ & $\mathrm{G}$ & $\mathrm{C}$ & $\mathrm{G}$ & $\mathrm{A}$ & $\bar{G}$ \\
\hline $\begin{array}{l}\text { East- } \\
\text { Asia } \\
\end{array}$ & China & $\underset{4}{\mathrm{CH}} \mathrm{CH} 2$ & $\mathrm{~T}$ & $\mathrm{C}$ & $\mathrm{T}$ & $\mathrm{G}$ & $\mathrm{C}$ & $\mathrm{G}$ & $\mathrm{C}$ & $\mathrm{A}$ & $\mathrm{G}$ & $\mathrm{T}$ & $\bar{A}$ & $\mathrm{G}$ & $\mathrm{G}$ & $\mathrm{C}$ & $\mathrm{G}$ & $\mathrm{A}$ & $\bar{G}$ \\
\hline $\begin{array}{l}\text { East- } \\
\text { Asia } \\
\end{array}$ & China & $\underset{4}{\mathrm{CH}+\mathrm{CH} 2}$ & $\mathrm{~T}$ & $\mathrm{C}$ & $\mathrm{T}$ & $\mathrm{G}$ & $\bar{C}$ & $\mathrm{G}$ & $\mathrm{C}$ & $\mathrm{A}$ & $\mathrm{G}$ & $\mathrm{T}$ & $\mathrm{A}$ & $\mathrm{G}$ & $\mathrm{G}$ & $\mathrm{C}$ & $\mathrm{G}$ & $\mathrm{A}$ & $\bar{G}$ \\
\hline $\begin{array}{l}\text { East- } \\
\text { Asia } \\
\end{array}$ & $\begin{array}{c}\text { Japane } \\
\text { se }\end{array}$ & JPN_J36 & $\mathrm{T}$ & $\mathrm{C}$ & $\mathrm{T}$ & $\mathrm{G}$ & $\mathrm{C}$ & $\mathrm{G}$ & $\mathrm{C}$ & $\mathrm{A}$ & $\mathrm{G}$ & $\mathrm{T}$ & $\mathrm{A}$ & $\mathrm{G}$ & $\mathrm{G}$ & $\mathrm{C}$ & $\mathrm{G}$ & $\mathrm{A}$ & $\bar{G}$ \\
\hline $\begin{array}{l}\text { East- } \\
\text { Asia } \\
\end{array}$ & \begin{tabular}{|c|}
$\begin{array}{c}\text { Japane } \\
\text { se }\end{array}$ \\
\end{tabular} & JPN_J36 & $\mathrm{T}$ & $\mathrm{C}$ & $\mathrm{T}$ & $\mathrm{G}$ & $\mathrm{C}$ & $\mathrm{G}$ & $\mathrm{C}$ & $\mathrm{A}$ & $\mathrm{G}$ & $\mathrm{T}$ & $\mathrm{A}$ & $\mathrm{G}$ & $\mathrm{G}$ & $\mathrm{C}$ & $\mathrm{G}$ & $\mathrm{A}$ & $\mathrm{G}$ \\
\hline $\begin{array}{l}\text { East- } \\
\text { Asia } \\
\end{array}$ & $\begin{array}{c}\text { Japane } \\
\text { se }\end{array}$ & JPN_J37 & $\mathrm{T}$ & $\mathrm{C}$ & $\mathrm{T}$ & $\mathrm{G}$ & $\mathrm{C}$ & $\mathrm{G}$ & $\mathrm{C}$ & $\mathrm{A}$ & $\mathrm{G}$ & $\mathrm{T}$ & $\mathrm{A}$ & $\mathrm{G}$ & $\mathrm{G}$ & $\mathrm{C}$ & $\mathrm{G}$ & $\mathrm{A}$ & $\bar{G}$ \\
\hline $\begin{array}{l}\text { East- } \\
\text { Asia } \\
\end{array}$ & $\begin{array}{c}\text { Japane } \\
\text { se }\end{array}$ & JPN_J37 & $\mathrm{T}$ & $\mathrm{C}$ & $\mathrm{T}$ & $\mathrm{G}$ & $\mathrm{C}$ & $\mathrm{G}$ & $\mathrm{C}$ & $\mathrm{A}$ & $\mathrm{G}$ & $\mathrm{T}$ & $\mathrm{A}$ & $\mathrm{G}$ & $\mathrm{G}$ & $\mathrm{C}$ & G & A & $\mathrm{G}$ \\
\hline $\begin{array}{l}\text { East- } \\
\text { Asia } \\
\end{array}$ & \begin{tabular}{|c|}
$\begin{array}{c}\text { Japane } \\
\text { se }\end{array}$ \\
\end{tabular} & JPN_J38 & $\mathrm{T}$ & $\mathrm{C}$ & $\mathrm{T}$ & $\mathrm{G}$ & $\mathrm{C}$ & $\mathrm{G}$ & $\mathrm{C}$ & $\mathrm{A}$ & $\mathrm{G}$ & $\mathrm{T}$ & $\mathrm{A}$ & $\mathrm{G}$ & $\mathrm{G}$ & $\mathrm{C}$ & $\mathrm{G}$ & $\mathrm{A}$ & $\mathrm{G}$ \\
\hline $\begin{array}{l}\text { East- } \\
\text { Asia } \\
\end{array}$ & $\begin{array}{c}\text { Japane } \\
\text { se }\end{array}$ & JPN_J38 & $\mathrm{T}$ & $\mathrm{C}$ & $\mathrm{T}$ & $\mathrm{G}$ & $\mathrm{C}$ & $\mathrm{G}$ & $\mathrm{C}$ & $\mathrm{A}$ & $\mathrm{G}$ & $\mathrm{T}$ & $\mathrm{A}$ & $\mathrm{G}$ & $\mathrm{G}$ & $\mathrm{C}$ & $\mathrm{G}$ & $\mathrm{A}$ & $\mathrm{G}$ \\
\hline $\begin{array}{l}\text { East- } \\
\text { Asia }\end{array}$ & $\begin{array}{c}\text { Japane } \\
\text { se }\end{array}$ & JPN_J39 & $\mathrm{T}$ & $\mathrm{C}$ & $\mathrm{T}$ & $\mathrm{G}$ & $\mathrm{C}$ & $\mathrm{G}$ & $\mathrm{C}$ & $\mathrm{A}$ & $\mathrm{G}$ & $\mathrm{T}$ & $\mathrm{A}$ & $\mathrm{G}$ & $\mathrm{G}$ & $\mathrm{C}$ & $\mathrm{G}$ & $\mathrm{A}$ & $\mathrm{G}$ \\
\hline
\end{tabular}

John Wiley 34 \& Sons, Inc. 


\begin{tabular}{|c|c|c|c|c|c|c|c|c|c|c|c|c|c|c|c|c|c|c|c|}
\hline $\begin{array}{l}\text { East- } \\
\text { Asia }\end{array}$ & $\begin{array}{c}\text { Japane } \\
\text { se }\end{array}$ & JPN_J39 & $\mathrm{T}$ & $\mathrm{C}$ & $\mathrm{T}$ & $\mathrm{G}$ & $\mathrm{C}$ & $\mathrm{G}$ & $\mathrm{C}$ & $\mathrm{A}$ & $\mathrm{G}$ & $\mathrm{T}$ & $\mathrm{A}$ & $\mathrm{G}$ & $\mathrm{G}$ & $\mathrm{C}$ & $\mathrm{G}$ & $\mathrm{A}$ & $\bar{G}$ \\
\hline $\begin{array}{l}\text { East- } \\
\text { Asia } \\
\end{array}$ & \begin{tabular}{|c|}
$\begin{array}{c}\text { Japane } \\
\text { se }\end{array}$ \\
\end{tabular} & JPN_J40 & $\mathrm{T}$ & $\mathrm{C}$ & $\mathrm{T}$ & $\mathrm{G}$ & $\mathrm{C}$ & $\mathrm{G}$ & $\mathrm{C}$ & $\mathrm{A}$ & $\mathrm{G}$ & $\mathrm{T}$ & A & $\mathrm{G}$ & $\mathrm{G}$ & $\mathrm{C}$ & G & $\mathrm{A}$ & $\mathrm{G}$ \\
\hline $\begin{array}{l}\text { East- } \\
\text { Asia } \\
\end{array}$ & $\begin{array}{c}\text { Japane } \\
\text { se }\end{array}$ & JPN_J40 & $\mathrm{T}$ & $\mathrm{C}$ & $\mathrm{T}$ & $\mathrm{G}$ & $\mathrm{C}$ & $\mathrm{G}$ & $\mathrm{C}$ & $\mathrm{A}$ & $\mathrm{G}$ & $\mathrm{T}$ & $\mathrm{A}$ & $\mathrm{G}$ & $\mathrm{G}$ & $\mathrm{C}$ & $\mathrm{T}$ & $\mathrm{A}$ & $\bar{G}$ \\
\hline $\begin{array}{l}\text { East- } \\
\text { Asia } \\
\end{array}$ & $\begin{array}{c}\text { Japane } \\
\text { se }\end{array}$ & JPN_J41 & $\mathrm{T}$ & $\mathrm{C}$ & $\mathrm{T}$ & $\mathrm{G}$ & $\mathrm{C}$ & $\mathrm{G}$ & $\mathrm{C}$ & $\mathrm{A}$ & $\mathrm{G}$ & $\mathrm{T}$ & A & $\mathrm{G}$ & $\mathrm{G}$ & $\mathrm{C}$ & $\mathrm{G}$ & $\mathrm{A}$ & $\mathrm{G}$ \\
\hline $\begin{array}{l}\text { East- } \\
\text { Asia } \\
\end{array}$ & \begin{tabular}{|c|}
$\begin{array}{c}\text { Japane } \\
\text { se }\end{array}$ \\
\end{tabular} & JPN_J41 & $\mathrm{T}$ & $\mathrm{C}$ & $\mathrm{T}$ & $\mathrm{G}$ & $\mathrm{C}$ & $\mathrm{G}$ & $\mathrm{C}$ & $\mathrm{A}$ & $\mathrm{G}$ & $\mathrm{T}$ & $\mathrm{A}$ & $\mathrm{G}$ & $\mathrm{G}$ & $\mathrm{C}$ & $\mathrm{G}$ & $\mathrm{A}$ & $\mathrm{G}$ \\
\hline $\begin{array}{l}\text { East- } \\
\text { Asia } \\
\end{array}$ & $\begin{array}{c}\text { Japane } \\
\text { se }\end{array}$ & JPN_J42 & $\mathrm{T}$ & $\mathrm{C}$ & $\mathrm{T}$ & $\mathrm{G}$ & $\mathrm{C}$ & $\mathrm{G}$ & $\mathrm{C}$ & $\mathrm{A}$ & $\mathrm{G}$ & $\mathrm{T}$ & $\mathrm{A}$ & $\mathrm{G}$ & $\mathrm{G}$ & $\mathrm{C}$ & $\mathrm{G}$ & A & G \\
\hline $\begin{array}{l}\text { East- } \\
\text { Asia } \\
\end{array}$ & $\begin{array}{c}\text { Japane } \\
\text { se }\end{array}$ & JPN_J42 & $\mathrm{T}$ & $\mathrm{C}$ & $\mathrm{T}$ & $\mathrm{G}$ & $\mathrm{C}$ & $\mathrm{G}$ & $\mathrm{C}$ & $\mathrm{A}$ & $\mathrm{G}$ & $\mathrm{T}$ & $\mathrm{A}$ & $\mathrm{G}$ & $\mathrm{G}$ & $\mathrm{C}$ & $\mathrm{T}$ & $\mathrm{A}$ & $\mathrm{G}$ \\
\hline $\begin{array}{l}\text { East- } \\
\text { Asia } \\
\end{array}$ & \begin{tabular}{|c|}
$\begin{array}{c}\text { Japane } \\
\text { se }\end{array}$ \\
\end{tabular} & $\begin{array}{c}\text { JPN_JK1 } \\
4 \overline{24} \\
\end{array}$ & $\mathrm{~T}$ & $\mathrm{C}$ & $\mathrm{T}$ & $\mathrm{G}$ & $\mathrm{C}$ & $\mathrm{G}$ & $\mathrm{C}$ & $\mathrm{A}$ & $\mathrm{G}$ & $\mathrm{T}$ & $\mathrm{A}$ & $\mathrm{G}$ & $\mathrm{G}$ & $\mathrm{C}$ & $\mathrm{G}$ & $\mathrm{A}$ & $\mathrm{G}$ \\
\hline $\begin{array}{l}\text { East- } \\
\text { Asia } \\
\end{array}$ & $\begin{array}{c}\text { Japane } \\
\text { se }\end{array}$ & $\begin{array}{c}\text { JPN_JK1 } \\
424\end{array}$ & $\mathrm{~T}$ & $\mathrm{C}$ & $\mathrm{T}$ & $\mathrm{G}$ & $\mathrm{C}$ & $\mathrm{G}$ & $\mathrm{C}$ & $\mathrm{A}$ & $\mathrm{G}$ & $\mathrm{T}$ & $\mathrm{A}$ & $\mathrm{G}$ & $\mathrm{G}$ & $\mathrm{C}$ & $\mathrm{G}$ & A & G \\
\hline $\begin{array}{l}\text { East- } \\
\text { Asia } \\
\end{array}$ & $\begin{array}{c}\text { Japane } \\
\text { se }\end{array}$ & $\begin{array}{c}\text { JPN_JK1 } \\
\mathbf{4 2 5}\end{array}$ & $\mathrm{T}$ & $\mathrm{C}$ & $\mathrm{T}$ & $\mathrm{G}$ & $\mathrm{C}$ & $\mathrm{G}$ & $\mathrm{C}$ & $\mathrm{A}$ & $\mathrm{G}$ & $\mathrm{T}$ & $\mathrm{A}$ & $\mathrm{G}$ & $\mathrm{G}$ & $\mathrm{C}$ & $\mathrm{G}$ & $\mathrm{A}$ & $\bar{G}$ \\
\hline $\begin{array}{l}\text { East- } \\
\text { Asia } \\
\end{array}$ & \begin{tabular}{|c} 
Japane \\
se
\end{tabular} & $\begin{array}{c}\text { JPN_JK1 } \\
425 \\
\end{array}$ & $\mathrm{~T}$ & $\mathrm{C}$ & $\mathrm{T}$ & $\mathrm{G}$ & $\mathrm{C}$ & $\mathrm{G}$ & $\mathrm{C}$ & $\mathrm{A}$ & $\mathrm{G}$ & $\mathrm{T}$ & $\mathrm{A}$ & $\mathrm{G}$ & $\mathrm{G}$ & $\mathrm{C}$ & $\mathrm{G}$ & $\mathrm{A}$ & $\bar{G}$ \\
\hline $\begin{array}{l}\text { East- } \\
\text { Asia } \\
\end{array}$ & $\begin{array}{c}\text { Japane } \\
\text { se }\end{array}$ & $\begin{array}{c}\text { JPN_JK1 } \\
426 \\
\end{array}$ & $\mathrm{~T}$ & $\mathrm{C}$ & $\mathrm{T}$ & G & $\mathrm{C}$ & $\mathrm{G}$ & $\mathrm{C}$ & $\mathrm{A}$ & $\mathrm{G}$ & $\mathrm{T}$ & $\mathrm{A}$ & $\mathrm{G}$ & $\mathrm{G}$ & $\mathrm{C}$ & $\mathrm{G}$ & $\mathrm{A}$ & G \\
\hline $\begin{array}{l}\text { East- } \\
\text { Asia } \\
\end{array}$ & $\begin{array}{c}\text { Japane } \\
\text { se }\end{array}$ & $\begin{array}{c}\text { JPN_JK1 } \\
426 \\
\end{array}$ & $\mathrm{~T}$ & $\mathrm{C}$ & $\mathrm{T}$ & $\mathrm{G}$ & $\bar{C}$ & $\mathrm{G}$ & $\mathrm{C}$ & $\mathrm{A}$ & $\mathrm{G}$ & $\mathrm{T}$ & $\mathrm{A}$ & $\mathrm{G}$ & $\mathrm{G}$ & $\mathrm{C}$ & $\mathrm{G}$ & $\overline{\mathrm{A}}$ & G \\
\hline $\begin{array}{l}\text { East- } \\
\text { Asia } \\
\end{array}$ & \begin{tabular}{|c} 
Japane \\
se
\end{tabular} & JPN_J30 & $\mathrm{T}$ & $\mathrm{C}$ & $\mathrm{T}$ & $\mathrm{G}$ & $\mathrm{C}$ & $\mathrm{G}$ & $\mathrm{C}$ & $\mathrm{A}$ & $\mathrm{G}$ & $\mathrm{T}$ & $\mathrm{A}$ & $\mathrm{G}$ & $\mathrm{G}$ & $\mathrm{C}$ & $\mathrm{G}$ & $\bar{A}$ & $\bar{G}$ \\
\hline $\begin{array}{l}\text { East- } \\
\text { Asia } \\
\end{array}$ & $\begin{array}{c}\text { Japane } \\
\text { se }\end{array}$ & JPN_J30 & $\mathrm{T}$ & $\mathrm{C}$ & $\mathrm{T}$ & $\mathrm{G}$ & $\mathrm{C}$ & $\mathrm{G}$ & $\mathrm{C}$ & $\mathrm{A}$ & $\mathrm{G}$ & $\mathrm{T}$ & $\mathrm{A}$ & $\mathrm{G}$ & $\mathrm{G}$ & $\mathrm{C}$ & $\mathrm{G}$ & $\mathrm{A}$ & $\mathrm{G}$ \\
\hline $\begin{array}{l}\text { East- } \\
\text { Asia } \\
\end{array}$ & $\begin{array}{c}\text { Japane } \\
\text { se }\end{array}$ & $\begin{array}{c}\text { JPN_JK1 } \\
785 \\
\end{array}$ & $\mathrm{~T}$ & $\mathrm{C}$ & $\mathrm{T}$ & $\mathrm{G}$ & $\bar{C}$ & $\mathrm{G}$ & $\mathrm{C}$ & $\mathrm{A}$ & $\mathrm{G}$ & $\mathrm{T}$ & $\mathrm{A}$ & $\mathrm{G}$ & $\mathrm{G}$ & $\mathrm{C}$ & $\mathrm{G}$ & $\bar{A}$ & $\mathrm{G}$ \\
\hline $\begin{array}{l}\text { East- } \\
\text { Asia } \\
\end{array}$ & $\begin{array}{c}\text { Japane } \\
\text { se }\end{array}$ & $\begin{array}{c}\text { JPN_JK1 } \\
785 \\
\end{array}$ & $\mathrm{~T}$ & $\mathrm{C}$ & $\mathrm{T}$ & $\mathrm{G}$ & $\mathrm{C}$ & $\mathrm{G}$ & $\mathrm{C}$ & $\mathrm{A}$ & $\mathrm{G}$ & $\mathrm{T}$ & $\mathrm{A}$ & $\mathrm{G}$ & $\mathrm{G}$ & $\mathrm{C}$ & $\mathrm{G}$ & $\mathrm{A}$ & $\mathrm{G}$ \\
\hline $\begin{array}{l}\text { East- } \\
\text { Asia } \\
\end{array}$ & \begin{tabular}{|c|}
$\begin{array}{c}\text { Japane } \\
\text { se }\end{array}$ \\
\end{tabular} & $\begin{array}{c}\text { JPN_JK1 } \\
788 \\
\end{array}$ & $\mathrm{~T}$ & $\mathrm{C}$ & $\mathrm{T}$ & $\mathrm{G}$ & $\mathrm{C}$ & $\mathrm{G}$ & $\mathrm{C}$ & $\mathrm{A}$ & $\mathrm{G}$ & $\mathrm{T}$ & $\mathrm{A}$ & $\mathrm{G}$ & $\mathrm{G}$ & $\mathrm{C}$ & $\mathrm{G}$ & $\mathrm{A}$ & $\mathrm{G}$ \\
\hline $\begin{array}{l}\text { East- } \\
\text { Asia } \\
\end{array}$ & $\begin{array}{c}\text { Japane } \\
\text { se }\end{array}$ & $\begin{array}{c}\text { JPN_JK1 } \\
788\end{array}$ & $\mathrm{~T}$ & $\mathrm{C}$ & $\mathrm{T}$ & $\mathrm{G}$ & $\mathrm{C}$ & $\mathrm{G}$ & $\mathrm{C}$ & $\mathrm{A}$ & $\mathrm{G}$ & $\mathrm{T}$ & $\mathrm{A}$ & $\mathrm{G}$ & $\mathrm{G}$ & $\mathrm{C}$ & $\mathrm{G}$ & $\mathrm{A}$ & $\mathrm{G}$ \\
\hline $\begin{array}{l}\text { East- } \\
\text { Asia } \\
\end{array}$ & $\begin{array}{c}\text { Japane } \\
\text { se }\end{array}$ & $\begin{array}{c}\text { JPN_JK1 } \\
790 \\
\end{array}$ & $\mathrm{~T}$ & $\mathrm{G}$ & $\mathrm{T}$ & $\mathrm{G}$ & $\mathrm{C}$ & $\mathrm{G}$ & $\mathrm{C}$ & A & $\mathrm{G}$ & $\mathrm{T}$ & $\mathrm{A}$ & $\mathrm{G}$ & $\mathrm{G}$ & $\mathrm{C}$ & $\mathrm{G}$ & $\mathrm{A}$ & $\mathrm{G}$ \\
\hline $\begin{array}{l}\text { East- } \\
\text { Asia } \\
\end{array}$ & \begin{tabular}{|c|}
$\begin{array}{c}\text { Japane } \\
\text { se }\end{array}$ \\
\end{tabular} & $\begin{array}{c}\text { JPN_JK1 } \\
790 \\
\end{array}$ & $\mathrm{~T}$ & $\mathrm{G}$ & $\mathrm{T}$ & $\mathrm{G}$ & $\mathrm{C}$ & $\mathrm{G}$ & $\mathrm{C}$ & $\mathrm{A}$ & $\mathrm{G}$ & $\mathrm{T}$ & $\mathrm{A}$ & $\mathrm{G}$ & $\mathrm{G}$ & $\mathrm{C}$ & $\mathrm{G}$ & $\mathrm{A}$ & $\mathrm{G}$ \\
\hline $\begin{array}{l}\text { East- } \\
\text { Asia } \\
\end{array}$ & $\begin{array}{c}\text { Japane } \\
\text { se }\end{array}$ & $\begin{array}{c}\text { JPN_JK1 } \\
793\end{array}$ & $\mathrm{~T}$ & $\mathrm{C}$ & $\mathrm{T}$ & $\mathrm{G}$ & $\mathrm{C}$ & $\mathrm{G}$ & $\mathrm{C}$ & $\mathrm{A}$ & $\mathrm{G}$ & $\mathrm{T}$ & $\mathrm{A}$ & $\mathrm{G}$ & $\mathrm{G}$ & $\mathrm{C}$ & $\mathrm{G}$ & $\mathrm{A}$ & $\mathrm{G}$ \\
\hline $\begin{array}{l}\text { East- } \\
\text { Asia } \\
\end{array}$ & $\begin{array}{c}\text { Japane } \\
\text { se }\end{array}$ & $\begin{array}{c}\text { JPN_JK1 } \\
793 \\
\end{array}$ & $\mathrm{~T}$ & $\mathrm{C}$ & $\mathrm{T}$ & $\mathrm{G}$ & $\mathrm{C}$ & $\mathrm{G}$ & $\mathrm{C}$ & A & $\mathrm{G}$ & $\mathrm{T}$ & $\mathrm{A}$ & $\mathrm{G}$ & $\mathrm{G}$ & $\mathrm{C}$ & $\mathrm{G}$ & $\mathrm{A}$ & $\mathrm{G}$ \\
\hline
\end{tabular}

John Wiley ${ }^{35}$ Sons, Inc. 


\begin{tabular}{|c|c|c|c|c|c|c|c|c|c|c|c|c|c|c|c|c|c|c|c|}
\hline $\begin{array}{l}\text { East- } \\
\text { Asia }\end{array}$ & $\begin{array}{c}\text { Japane } \\
\text { se }\end{array}$ & $\begin{array}{c}\text { JPN_JK1 } \\
850\end{array}$ & $\mathrm{~T}$ & $\bar{C}$ & $\mathrm{~T}$ & $\mathrm{G}$ & $\mathrm{C}$ & $\mathrm{G}$ & $\mathrm{C}$ & $\mathrm{A}$ & $\mathrm{G}$ & $\mathrm{T}$ & $\mathrm{A}$ & $\bar{G}$ & $\mathrm{G}$ & $\mathrm{C}$ & $\mathrm{G}$ & $\mathrm{A}$ & $\overline{\mathrm{G}}$ \\
\hline $\begin{array}{l}\text { East- } \\
\text { Asia } \\
\end{array}$ & $\begin{array}{c}\text { Japane } \\
\text { se }\end{array}$ & $\begin{array}{c}\text { JPN_JK1 } \\
850\end{array}$ & $\mathrm{~T}$ & $\mathrm{C}$ & $\mathrm{T}$ & $\mathrm{G}$ & $\mathrm{C}$ & $\mathrm{G}$ & $\mathrm{C}$ & $\mathrm{A}$ & $\mathrm{G}$ & $\mathrm{T}$ & $\mathrm{A}$ & $\mathrm{G}$ & $\mathrm{G}$ & $\mathrm{C}$ & $\mathrm{T}$ & $\mathrm{A}$ & $\mathrm{G}$ \\
\hline $\begin{array}{l}\text { East- } \\
\text { Asia } \\
\end{array}$ & $\begin{array}{c}\text { Japane } \\
\text { se }\end{array}$ & $\begin{array}{c}\text { JPN_JK1 } \\
851 \\
\end{array}$ & $\mathrm{~T}$ & $\mathrm{C}$ & $\mathrm{T}$ & $\mathrm{G}$ & $\bar{C}$ & $\mathrm{G}$ & $\mathrm{C}$ & $\mathrm{A}$ & $\mathrm{G}$ & $\mathrm{T}$ & $\mathrm{A}$ & $\bar{G}$ & $\mathrm{G}$ & $\mathrm{C}$ & $\mathrm{G}$ & $\mathrm{A}$ & $\bar{G}$ \\
\hline $\begin{array}{l}\text { East- } \\
\text { Asia } \\
\end{array}$ & $\begin{array}{c}\text { Japane } \\
\text { se }\end{array}$ & $\begin{array}{c}\text { JPN_JK1 } \\
851 \\
\end{array}$ & $\mathrm{~T}$ & $\mathrm{C}$ & $\mathrm{T}$ & $\mathrm{G}$ & $\mathrm{C}$ & $\mathrm{G}$ & $\mathrm{C}$ & $\mathrm{A}$ & $\mathrm{G}$ & $\mathrm{T}$ & $\mathrm{A}$ & $\mathrm{G}$ & $\mathrm{G}$ & $\mathrm{C}$ & $\mathrm{T}$ & $\mathrm{A}$ & $\mathrm{G}$ \\
\hline $\begin{array}{l}\text { East- } \\
\text { Asia } \\
\end{array}$ & \begin{tabular}{|c|} 
Japane \\
se
\end{tabular} & $\begin{array}{c}\text { JPN_JK1 } \\
852 \\
\end{array}$ & $\mathrm{~T}$ & $\mathrm{C}$ & $\mathrm{T}$ & $\mathrm{G}$ & $\mathrm{C}$ & $\mathrm{G}$ & $\mathrm{C}$ & $\mathrm{A}$ & $\mathrm{G}$ & $\mathrm{T}$ & $\mathrm{A}$ & $\bar{G}$ & $\mathrm{G}$ & $\mathrm{C}$ & $\mathrm{G}$ & $\mathrm{A}$ & $\bar{G}$ \\
\hline $\begin{array}{l}\text { East- } \\
\text { Asia } \\
\end{array}$ & $\begin{array}{c}\text { Japane } \\
\text { se }\end{array}$ & $\begin{array}{c}\text { JPN_JK1 } \\
852 \\
\end{array}$ & $\mathrm{~T}$ & $\mathrm{C}$ & $\mathrm{T}$ & $\mathrm{G}$ & $\mathrm{C}$ & $\mathrm{G}$ & $\mathrm{C}$ & $\mathrm{A}$ & $\mathrm{G}$ & $\mathrm{T}$ & $\mathrm{A}$ & $\mathrm{G}$ & $\mathrm{G}$ & $\mathrm{C}$ & $\mathrm{T}$ & $\mathrm{A}$ & $\mathrm{G}$ \\
\hline $\begin{array}{l}\text { East- } \\
\text { Asia }\end{array}$ & $\begin{array}{c}\text { Japane } \\
\text { se }\end{array}$ & $\begin{array}{c}\text { JPN_JK1 } \\
853\end{array}$ & $\mathrm{~T}$ & $\mathrm{C}$ & $\mathrm{T}$ & $\mathrm{G}$ & $\mathrm{C}$ & $\mathrm{G}$ & $\mathrm{C}$ & $\mathrm{A}$ & $\mathrm{G}$ & $\mathrm{T}$ & $\mathrm{A}$ & $\mathrm{G}$ & $\mathrm{G}$ & $\mathrm{C}$ & $\mathrm{G}$ & $\mathrm{A}$ & $\bar{G}$ \\
\hline $\begin{array}{l}\text { East- } \\
\text { Asia } \\
\end{array}$ & \begin{tabular}{|c|}
$\begin{array}{c}\text { Japane } \\
\text { se }\end{array}$ \\
\end{tabular} & $\begin{array}{c}\text { JPN_JK1 } \\
853 \\
\end{array}$ & $\mathrm{~T}$ & $\mathrm{C}$ & $\mathrm{T}$ & $\mathrm{G}$ & $\mathrm{C}$ & $\mathrm{G}$ & $\mathrm{C}$ & $\mathrm{A}$ & $\mathrm{G}$ & $\mathrm{T}$ & $\mathrm{A}$ & G & $\mathrm{G}$ & $\mathrm{C}$ & $\mathrm{T}$ & $\mathrm{A}$ & $\bar{G}$ \\
\hline $\begin{array}{l}\text { East- } \\
\text { Asia } \\
\end{array}$ & $\begin{array}{c}\text { Japane } \\
\text { se }\end{array}$ & $\begin{array}{c}\text { JPN_JK1 } \\
856\end{array}$ & $\mathrm{~T}$ & $\mathrm{C}$ & $\mathrm{T}$ & $\mathrm{G}$ & $\mathrm{C}$ & $\mathrm{G}$ & $\mathrm{C}$ & $\mathrm{A}$ & $\mathrm{G}$ & $\mathrm{T}$ & $\mathrm{A}$ & G & $\mathrm{G}$ & $\mathrm{C}$ & $\mathrm{G}$ & $\mathrm{A}$ & $\mathrm{G}$ \\
\hline $\begin{array}{l}\text { East- } \\
\text { Asia }\end{array}$ & $\begin{array}{c}\text { Japane } \\
\text { se }\end{array}$ & $\begin{array}{c}\text { JPN_JK1 } \\
856\end{array}$ & $\mathrm{~T}$ & $\mathrm{C}$ & $\mathrm{T}$ & $\mathrm{G}$ & $\mathrm{C}$ & $\mathrm{G}$ & $\mathrm{C}$ & $\mathrm{A}$ & $\mathrm{G}$ & $\mathrm{T}$ & $\mathrm{A}$ & $\mathrm{G}$ & $\mathrm{G}$ & $\mathrm{C}$ & $\mathrm{T}$ & $\mathrm{A}$ & $\overline{\mathrm{G}}$ \\
\hline $\begin{array}{l}\text { East- } \\
\text { Asia } \\
\end{array}$ & $\begin{array}{c}\text { Japane } \\
\text { se }\end{array}$ & $\begin{array}{c}\text { JPN_JK1 } \\
857 \\
\end{array}$ & $\mathrm{~T}$ & $\mathrm{C}$ & $\mathrm{T}$ & $\mathrm{G}$ & $\mathrm{C}$ & $\mathrm{G}$ & $\mathrm{C}$ & $\mathrm{A}$ & $\mathrm{G}$ & $\mathrm{T}$ & $\mathrm{A}$ & $\bar{G}$ & $\mathrm{G}$ & $\mathrm{C}$ & $\mathrm{G}$ & $\bar{A}$ & $\overline{\mathrm{G}}$ \\
\hline $\begin{array}{l}\text { East- } \\
\text { Asia } \\
\end{array}$ & $\begin{array}{c}\text { Japane } \\
\text { se }\end{array}$ & $\begin{array}{c}\text { JPN_JK1 } \\
857\end{array}$ & $\mathrm{~T}$ & $\mathrm{C}$ & $\mathrm{T}$ & $\mathrm{G}$ & $\mathrm{C}$ & $\mathrm{G}$ & $\mathrm{C}$ & $\mathrm{A}$ & $\mathrm{G}$ & $\mathrm{T}$ & $\mathrm{A}$ & $\mathrm{G}$ & $\mathrm{G}$ & $\mathrm{C}$ & $\mathrm{G}$ & $\mathrm{A}$ & $\mathrm{G}$ \\
\hline $\begin{array}{l}\text { East- } \\
\text { Asia }\end{array}$ & $\begin{array}{c}\text { Japane } \\
\text { se }\end{array}$ & $\begin{array}{c}\text { JPN_JK1 } \\
860\end{array}$ & $\mathrm{C}$ & $\mathrm{C}$ & $\mathrm{T}$ & $\mathrm{G}$ & $\mathrm{C}$ & $\mathrm{G}$ & $\mathrm{C}$ & $\mathrm{A}$ & $\mathrm{A}$ & $\mathrm{T}$ & $\mathrm{A}$ & $\bar{G}$ & $\mathrm{G}$ & $\mathrm{C}$ & $\mathrm{G}$ & $\mathrm{A}$ & $\overline{\mathrm{G}}$ \\
\hline $\begin{array}{l}\text { East- } \\
\text { Asia } \\
\end{array}$ & $\begin{array}{c}\text { Japane } \\
\text { se }\end{array}$ & $\begin{array}{c}\text { JPN_JK1 } \\
860 \\
\end{array}$ & $\mathrm{~T}$ & $\bar{C}$ & $\mathrm{~T}$ & $\mathrm{G}$ & $\mathrm{C}$ & $\mathrm{G}$ & $\mathrm{C}$ & $\mathrm{A}$ & $\mathrm{G}$ & $\mathrm{T}$ & $\mathrm{A}$ & $\bar{G}$ & $\mathrm{G}$ & $\mathrm{C}$ & $\mathrm{G}$ & $\bar{A}$ & $\bar{G}$ \\
\hline $\begin{array}{l}\text { East- } \\
\text { Asia } \\
\end{array}$ & $\begin{array}{c}\text { Japane } \\
\text { se }\end{array}$ & $\begin{array}{c}\text { JPN_JK1 } \\
863\end{array}$ & $\mathrm{~T}$ & $\mathrm{C}$ & $\mathrm{T}$ & $\mathrm{G}$ & $\mathrm{C}$ & $\mathrm{G}$ & $\mathrm{C}$ & $\mathrm{A}$ & $\mathrm{G}$ & $\mathrm{T}$ & $\mathrm{A}$ & $\mathrm{G}$ & $\mathrm{G}$ & $\bar{C}$ & $\mathrm{G}$ & $\mathrm{A}$ & $\bar{G}$ \\
\hline $\begin{array}{l}\text { East- } \\
\text { Asia } \\
\end{array}$ & $\begin{array}{c}\text { Japane } \\
\text { se }\end{array}$ & $\begin{array}{c}\text { JPN_JK1 } \\
863 \\
\end{array}$ & $\bar{T}$ & $\mathrm{C}$ & $\mathrm{T}$ & $\mathrm{G}$ & $\mathrm{C}$ & $\mathrm{G}$ & $\mathrm{C}$ & $\mathrm{A}$ & $\mathrm{G}$ & $\mathrm{T}$ & $\mathrm{A}$ & $\overline{\mathrm{A}}$ & $\mathrm{G}$ & $\mathrm{C}$ & $\mathrm{G}$ & $\bar{A}$ & $\bar{G}$ \\
\hline $\begin{array}{l}\text { East- } \\
\text { Asia } \\
\end{array}$ & $\begin{array}{c}\text { Japane } \\
\text { se }\end{array}$ & 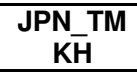 & $\mathrm{T}$ & $\mathrm{C}$ & $\mathrm{T}$ & $\mathrm{G}$ & $\mathrm{C}$ & $\mathrm{G}$ & $\bar{C}$ & $\mathrm{~A}$ & $\mathrm{G}$ & $\mathrm{T}$ & $\mathrm{A}$ & $\bar{G}$ & $\mathrm{G}$ & $\mathrm{C}$ & $\mathrm{G}$ & $\mathrm{A}$ & $\bar{G}$ \\
\hline $\begin{array}{l}\text { East- } \\
\text { Asia } \\
\end{array}$ & \begin{tabular}{|c|} 
Japane \\
se
\end{tabular} & $\begin{array}{c}\text { JPN_TM } \\
\mathbf{K} \overline{\mathbf{H}} \\
\end{array}$ & $\mathrm{T}$ & $\mathrm{C}$ & $\mathrm{T}$ & $\mathrm{G}$ & $\mathrm{C}$ & $\mathrm{G}$ & $\mathrm{C}$ & $\mathrm{A}$ & $\mathrm{G}$ & $\mathrm{T}$ & $\mathrm{A}$ & G & $\mathrm{G}$ & $\mathrm{C}$ & $\mathrm{G}$ & $\mathrm{A}$ & $\mathrm{G}$ \\
\hline $\begin{array}{c}\text { Europ } \\
\mathrm{e}\end{array}$ & $\begin{array}{c}\text { Chuvas } \\
\mathrm{h}\end{array}$ & $\begin{array}{c}\text { CHU_JK } \\
4051\end{array}$ & $\mathrm{~T}$ & $\mathrm{C}$ & $\mathrm{T}$ & $\mathrm{G}$ & $\mathrm{C}$ & $\mathrm{G}$ & $\mathrm{C}$ & $\mathrm{A}$ & $\mathrm{G}$ & $\mathrm{T}$ & $\mathrm{A}$ & G & $\mathrm{G}$ & $\mathrm{C}$ & $\mathrm{G}$ & $\mathrm{A}$ & $\mathrm{G}$ \\
\hline $\begin{array}{c}\text { Europ } \\
\mathrm{e}\end{array}$ & \begin{tabular}{|c}
$\begin{array}{c}\text { Chuvas } \\
\mathrm{h}\end{array}$ \\
\end{tabular} & $\begin{array}{c}\text { CHU_JK } \\
4051\end{array}$ & $\mathrm{~T}$ & $\mathrm{C}$ & $\mathrm{T}$ & $\mathrm{G}$ & $\mathrm{C}$ & $\mathrm{G}$ & $\bar{C}$ & $\mathrm{~A}$ & $\mathrm{G}$ & $\mathrm{T}$ & $\mathrm{A}$ & G & $\mathrm{G}$ & $\mathrm{C}$ & G & $\mathrm{A}$ & $\mathrm{G}$ \\
\hline $\begin{array}{c}\text { Europ } \\
\mathrm{e}\end{array}$ & \begin{tabular}{|c|c|}
$\begin{array}{c}\text { Chuvas } \\
\mathrm{h}\end{array}$ \\
\end{tabular} & $\begin{array}{c}\text { CHU_JK } \\
4052\end{array}$ & $\mathrm{~T}$ & $\mathrm{C}$ & $\mathrm{T}$ & $\mathrm{G}$ & $\mathrm{C}$ & $\mathrm{G}$ & $\bar{C}$ & $\mathrm{~A}$ & $\mathrm{G}$ & $\mathrm{T}$ & $\mathrm{A}$ & G & $\mathrm{G}$ & $\mathrm{C}$ & $\mathrm{G}$ & $\mathrm{A}$ & $\mathrm{G}$ \\
\hline $\begin{array}{c}\text { Europ } \\
\mathrm{e}\end{array}$ & $\begin{array}{c}\text { Chuvas } \\
\mathrm{h}\end{array}$ & $\begin{array}{c}\text { CHU_JK } \\
4052\end{array}$ & $\mathrm{~T}$ & $\mathrm{C}$ & $\mathrm{T}$ & $\mathrm{G}$ & $\mathrm{C}$ & $\mathrm{G}$ & $\mathrm{T}$ & $\mathrm{A}$ & $\mathrm{G}$ & $\mathrm{T}$ & $\mathrm{A}$ & $\mathrm{G}$ & $\mathrm{G}$ & $\mathrm{C}$ & $\mathrm{G}$ & $\mathrm{A}$ & $\mathrm{G}$ \\
\hline $\begin{array}{c}\text { Europ } \\
\mathrm{e}\end{array}$ & $\begin{array}{c}\text { Chuvas } \\
\text { h }\end{array}$ & $\begin{array}{c}\text { CHU_JK } \\
4053\end{array}$ & $\mathrm{~T}$ & $\mathrm{C}$ & $\mathrm{T}$ & $\mathrm{G}$ & $\mathrm{C}$ & $\mathrm{G}$ & $\mathrm{T}$ & $\mathrm{A}$ & $\mathrm{G}$ & $\mathrm{T}$ & $\mathrm{A}$ & $\mathrm{G}$ & $\mathrm{G}$ & $\mathrm{C}$ & $\mathrm{G}$ & $\mathrm{A}$ & $\bar{G}$ \\
\hline
\end{tabular}

\author{
John Wiley ${ }^{36} \&$ Sons, Inc.
}




\begin{tabular}{|c|c|c|c|c|c|c|c|c|c|c|c|c|c|c|c|c|c|c|c|}
\hline $\begin{array}{c}\text { Europ } \\
\mathrm{e}\end{array}$ & $\begin{array}{c}\text { Chuvas } \\
\mathrm{h}\end{array}$ & $\begin{array}{c}\text { CHU_JK } \\
4053\end{array}$ & $\mathrm{~T}$ & $\mathrm{C}$ & $\mathrm{T}$ & $\mathrm{G}$ & $\mathrm{C}$ & $\mathrm{G}$ & $\mathrm{T}$ & $\mathrm{A}$ & $\mathrm{G}$ & $\mathrm{T}$ & $\overline{\mathrm{A}}$ & $\mathrm{G}$ & $\mathrm{G}$ & $\mathrm{C}$ & $\mathrm{G}$ & $\mathrm{A}$ & $\bar{G}$ \\
\hline $\begin{array}{c}\text { Europ } \\
\mathrm{e}\end{array}$ & $\begin{array}{c}\text { Chuvas } \\
\mathrm{h}\end{array}$ & $\begin{array}{c}\text { CHU_JK } \\
4054\end{array}$ & $\mathrm{~T}$ & $\mathrm{C}$ & $\mathrm{T}$ & $\mathrm{G}$ & $\mathrm{C}$ & $\mathrm{G}$ & $\mathrm{C}$ & $\mathrm{A}$ & $\mathrm{G}$ & $\mathrm{T}$ & $\mathrm{A}$ & $\mathrm{G}$ & $\mathrm{G}$ & $\mathrm{C}$ & $\mathrm{G}$ & $\mathrm{A}$ & $\mathrm{G}$ \\
\hline $\begin{array}{c}\text { Europ } \\
\mathrm{e}\end{array}$ & $\begin{array}{c}\text { Chuvas } \\
\mathrm{h}\end{array}$ & $\underset{4054}{\text { CHU_JK }}$ & $\mathrm{T}$ & $\mathrm{C}$ & $\mathrm{T}$ & $\mathrm{G}$ & $\mathrm{C}$ & $\mathrm{G}$ & $\mathrm{C}$ & $\overline{\mathrm{A}}$ & $\mathrm{G}$ & $\mathrm{T}$ & $\bar{A}$ & $\mathrm{G}$ & $\mathrm{G}$ & $\mathrm{C}$ & $\mathrm{G}$ & $\mathrm{A}$ & $\mathrm{G}$ \\
\hline $\begin{array}{c}\text { Europ } \\
\mathrm{e}\end{array}$ & $\begin{array}{c}\text { Chuvas } \\
\mathrm{h}\end{array}$ & $\begin{array}{c}\text { CHU_JK } \\
4055\end{array}$ & $\mathrm{~T}$ & $\mathrm{C}$ & $\mathrm{T}$ & $\mathrm{G}$ & $\mathrm{C}$ & $\mathrm{G}$ & $\mathrm{T}$ & $\mathrm{A}$ & $\mathrm{G}$ & $\mathrm{T}$ & $\mathrm{A}$ & $\mathrm{G}$ & $\mathrm{G}$ & $\mathrm{C}$ & $\mathrm{G}$ & $\mathrm{A}$ & $\mathrm{G}$ \\
\hline $\begin{array}{c}\text { Europ } \\
\mathrm{e}\end{array}$ & $\begin{array}{c}\text { Chuvas } \\
\mathrm{h}\end{array}$ & $\begin{array}{c}\text { CHU_JK } \\
4055\end{array}$ & $\mathrm{~T}$ & $\mathrm{C}$ & $\mathrm{T}$ & $\mathrm{G}$ & $\mathrm{C}$ & $\mathrm{G}$ & $\mathrm{T}$ & $\mathrm{A}$ & $\mathrm{G}$ & $\mathrm{T}$ & $\mathrm{A}$ & $\mathrm{G}$ & $\mathrm{G}$ & $\mathrm{C}$ & $\mathrm{G}$ & $\mathrm{A}$ & $\mathrm{G}$ \\
\hline $\begin{array}{c}\text { Europ } \\
\mathrm{e}\end{array}$ & $\begin{array}{c}\text { Chuvas } \\
\mathrm{h}\end{array}$ & $\underset{4056}{\text { CHU_JK }}$ & $\mathrm{T}$ & $\mathrm{C}$ & $\mathrm{T}$ & $\mathrm{G}$ & $\mathrm{C}$ & $\mathrm{G}$ & $\mathrm{C}$ & $\bar{A}$ & $\mathrm{G}$ & $\mathrm{T}$ & $\mathrm{A}$ & $\mathrm{G}$ & $\mathrm{G}$ & $\mathrm{C}$ & $\mathrm{G}$ & $\mathrm{A}$ & $\mathrm{G}$ \\
\hline $\begin{array}{c}\text { Europ } \\
\mathrm{e}\end{array}$ & $\begin{array}{c}\text { Chuvas } \\
\mathrm{h}\end{array}$ & $\begin{array}{c}\text { CHU_JK } \\
4056\end{array}$ & $\mathrm{~T}$ & $\mathrm{C}$ & $\mathrm{T}$ & $\mathrm{G}$ & $\mathrm{C}$ & $\mathrm{G}$ & $\mathrm{C}$ & $\bar{A}$ & $\bar{G}$ & $\bar{T}$ & $\mathrm{~A}$ & $\mathrm{G}$ & $\bar{G}$ & $\mathrm{C}$ & $\mathrm{G}$ & $\mathrm{A}$ & $\overline{\mathrm{G}}$ \\
\hline $\begin{array}{c}\text { Europ } \\
\mathrm{e}\end{array}$ & $\begin{array}{c}\text { Chuvas } \\
\text { h }\end{array}$ & $\begin{array}{c}\text { CHU_JK } \\
4057\end{array}$ & $\mathrm{~T}$ & $\mathrm{C}$ & $\mathrm{T}$ & $\mathrm{G}$ & $\mathrm{C}$ & $\mathrm{G}$ & $\mathrm{C}$ & $\mathrm{A}$ & $\mathrm{G}$ & $\mathrm{T}$ & $\mathrm{A}$ & $\mathrm{G}$ & $\mathrm{G}$ & $\mathrm{C}$ & $\mathrm{G}$ & $\mathrm{A}$ & $\mathrm{G}$ \\
\hline $\begin{array}{c}\text { Europ } \\
\mathrm{e}\end{array}$ & $\begin{array}{c}\text { Chuvas } \\
\mathrm{h}\end{array}$ & $\underset{4057}{\text { CHU_JK }}$ & $\mathrm{T}$ & $\mathrm{C}$ & $\mathrm{T}$ & $\mathrm{G}$ & $\mathrm{C}$ & $\mathrm{G}$ & $\mathrm{C}$ & $\mathrm{A}$ & $\mathrm{G}$ & $\mathrm{T}$ & $\mathrm{A}$ & $\mathrm{G}$ & $\mathrm{G}$ & $\mathrm{C}$ & $\mathrm{G}$ & $\mathrm{A}$ & $\mathrm{G}$ \\
\hline $\begin{array}{c}\text { Europ } \\
\mathrm{e}\end{array}$ & $\begin{array}{c}\text { Chuvas } \\
\mathrm{h}\end{array}$ & $\underset{4058}{\text { CHU_JK }}$ & $\mathrm{T}$ & $\mathrm{C}$ & $\mathrm{T}$ & $\mathrm{G}$ & $\mathrm{C}$ & $\mathrm{G}$ & $\mathrm{C}$ & $\mathrm{A}$ & $\mathrm{G}$ & $\mathrm{T}$ & $\mathrm{A}$ & $\mathrm{G}$ & $\mathrm{G}$ & $\mathrm{C}$ & $\mathrm{G}$ & $\mathrm{A}$ & $\bar{G}$ \\
\hline $\begin{array}{c}\text { Europ } \\
\mathrm{e}\end{array}$ & $\begin{array}{c}\text { Chuvas } \\
\mathrm{h}\end{array}$ & $\begin{array}{c}\text { CHU_JK } \\
4058\end{array}$ & $\mathrm{~T}$ & $\mathrm{C}$ & $\mathrm{T}$ & $\mathrm{G}$ & $\mathrm{C}$ & $\mathrm{G}$ & $\mathrm{C}$ & $\mathrm{A}$ & $\mathrm{G}$ & $\mathrm{T}$ & $\mathrm{A}$ & $\mathrm{G}$ & $\mathrm{G}$ & $\mathrm{C}$ & $\mathrm{G}$ & $\mathrm{A}$ & $\overline{\mathrm{G}}$ \\
\hline $\begin{array}{c}\text { Europ } \\
\mathrm{e}\end{array}$ & $\begin{array}{c}\text { Chuvas } \\
\mathrm{h}\end{array}$ & $\underset{4059}{\text { CHU_JK }}$ & $\mathrm{T}$ & $\mathrm{C}$ & $\mathrm{T}$ & $\mathrm{G}$ & $\mathrm{C}$ & $\mathrm{G}$ & $\mathrm{T}$ & A & $\mathrm{G}$ & $\mathrm{T}$ & $\mathrm{A}$ & $\mathrm{G}$ & $\mathrm{G}$ & $\mathrm{C}$ & $\mathrm{G}$ & $\mathrm{A}$ & $\bar{G}$ \\
\hline $\begin{array}{c}\text { Europ } \\
\mathrm{e}\end{array}$ & $\begin{array}{c}\text { Chuvas } \\
\mathrm{h}\end{array}$ & $\underset{4059}{\text { CHU_JK }}$ & $\mathrm{T}$ & $\mathrm{C}$ & $\mathrm{T}$ & $\mathrm{G}$ & $\mathrm{C}$ & $\mathrm{G}$ & $\mathrm{C}$ & $\overline{\mathrm{A}}$ & $\bar{G}$ & $\mathrm{~T}$ & $\mathrm{~A}$ & $\mathrm{G}$ & $\bar{G}$ & $\mathrm{C}$ & $\mathrm{G}$ & $\mathrm{A}$ & $\overline{\mathrm{G}}$ \\
\hline $\begin{array}{c}\text { Europ } \\
\mathrm{e}\end{array}$ & $\begin{array}{c}\text { Chuvas } \\
\mathrm{h}\end{array}$ & $\begin{array}{c}\text { CHU_JK } \\
4060\end{array}$ & $\mathrm{~T}$ & $\mathrm{C}$ & $\mathrm{T}$ & $\mathrm{G}$ & $\mathrm{C}$ & $\mathrm{G}$ & $\mathrm{C}$ & $\overline{\mathrm{A}}$ & $\mathrm{G}$ & $\mathrm{T}$ & $\mathrm{A}$ & $\mathrm{G}$ & $\mathrm{G}$ & $\mathrm{C}$ & $\mathrm{G}$ & $\mathrm{A}$ & $\bar{G}$ \\
\hline $\begin{array}{c}\text { Europ } \\
\mathrm{e}\end{array}$ & $\begin{array}{c}\text { Chuvas } \\
\mathrm{h}\end{array}$ & $\underset{4060}{\mathrm{CHU} J \mathrm{JK}}$ & $\mathrm{T}$ & $\mathrm{C}$ & $\mathrm{T}$ & $\mathrm{G}$ & $\mathrm{C}$ & $\mathrm{G}$ & $\mathrm{C}$ & $\bar{A}$ & $\mathrm{G}$ & $\mathrm{T}$ & $\mathrm{A}$ & $\mathrm{G}$ & $\mathrm{G}$ & $\mathrm{C}$ & $\mathrm{G}$ & $\mathrm{A}$ & $\overline{\mathrm{G}}$ \\
\hline $\begin{array}{c}\text { Europ } \\
\mathrm{e}\end{array}$ & $\begin{array}{c}\text { Chuvas } \\
\mathrm{h}\end{array}$ & $\underset{4061}{\mathrm{CHU} J \mathrm{KK}}$ & $\mathrm{T}$ & $\mathrm{C}$ & $\mathrm{T}$ & $\mathrm{G}$ & $\mathrm{C}$ & $\mathrm{G}$ & $\mathrm{C}$ & $\mathrm{A}$ & $\mathrm{G}$ & $\mathrm{T}$ & $\mathrm{A}$ & $\mathrm{G}$ & $\mathrm{G}$ & $\mathrm{C}$ & $\mathrm{G}$ & $\mathrm{A}$ & $\mathrm{G}$ \\
\hline $\begin{array}{c}\text { Europ } \\
\mathrm{e}\end{array}$ & $\begin{array}{c}\text { Chuvas } \\
\mathrm{h}\end{array}$ & $\begin{array}{c}\text { CHU_JK } \\
4061\end{array}$ & $\mathrm{~T}$ & $\mathrm{C}$ & $\mathrm{T}$ & $\mathrm{G}$ & $\mathrm{C}$ & $\mathrm{G}$ & $\mathrm{C}$ & $\bar{A}$ & $\bar{G}$ & $\mathrm{~T}$ & $\mathrm{~A}$ & $\mathrm{G}$ & $\bar{G}$ & $\mathrm{C}$ & $\mathrm{G}$ & $\mathrm{A}$ & $\overline{\mathrm{G}}$ \\
\hline $\begin{array}{c}\text { Europ } \\
\mathrm{e}\end{array}$ & $\begin{array}{c}\text { Chuvas } \\
\mathrm{h}\end{array}$ & $\underset{4062}{\mathrm{CHU} J \mathrm{JK}}$ & $\mathrm{T}$ & $\mathrm{C}$ & $\mathrm{T}$ & $\mathrm{G}$ & $\mathrm{C}$ & $\mathrm{G}$ & $\mathrm{C}$ & $\mathrm{A}$ & $\mathrm{G}$ & $\mathrm{T}$ & A & $\mathrm{G}$ & $\mathrm{G}$ & $\mathrm{C}$ & $\mathrm{G}$ & $\mathrm{A}$ & $\mathrm{G}$ \\
\hline $\begin{array}{c}\text { Europ } \\
\mathrm{e}\end{array}$ & $\begin{array}{c}\text { Chuvas } \\
\mathrm{h}\end{array}$ & $\underset{4062}{\mathrm{CHU} J \mathrm{JK}}$ & $\mathrm{T}$ & $\mathrm{C}$ & $\mathrm{T}$ & $\mathrm{G}$ & $\mathrm{C}$ & $\mathrm{G}$ & $\mathrm{T}$ & $\mathrm{A}$ & $\mathrm{G}$ & $\mathrm{T}$ & $\mathrm{A}$ & $\mathrm{G}$ & $\mathrm{G}$ & $\mathrm{C}$ & $\mathrm{G}$ & $\mathrm{A}$ & $\mathrm{G}$ \\
\hline $\begin{array}{c}\text { Europ } \\
\mathrm{e}\end{array}$ & $\begin{array}{c}\text { Chuvas } \\
\mathrm{h}\end{array}$ & $\underset{4063}{\text { CHU_JK }}$ & $\mathrm{T}$ & $\mathrm{C}$ & $\mathrm{T}$ & $\mathrm{G}$ & $\mathrm{C}$ & $\mathrm{G}$ & $\mathrm{C}$ & $\bar{A}$ & $\bar{G}$ & $\mathrm{~T}$ & $\mathrm{~A}$ & $\mathrm{G}$ & $\mathrm{G}$ & $\mathrm{C}$ & $\mathrm{G}$ & $\mathrm{A}$ & $\bar{G}$ \\
\hline $\begin{array}{c}\text { Europ } \\
\mathrm{e}\end{array}$ & $\begin{array}{c}\text { Chuvas } \\
\mathrm{h}\end{array}$ & $\begin{array}{c}\text { CHU_JK } \\
4063\end{array}$ & $\mathrm{~T}$ & $\mathrm{C}$ & $\mathrm{T}$ & $\mathrm{G}$ & $\mathrm{C}$ & $\mathrm{G}$ & $\mathrm{C}$ & A & $\mathrm{G}$ & $\mathrm{T}$ & $\mathrm{A}$ & $\mathrm{G}$ & $\mathrm{G}$ & $\mathrm{C}$ & $\mathrm{G}$ & $\mathrm{A}$ & $\mathrm{G}$ \\
\hline $\begin{array}{c}\text { Europ } \\
\mathrm{e}\end{array}$ & $\begin{array}{c}\text { Chuvas } \\
\mathrm{h}\end{array}$ & $\underset{4064}{\mathrm{CHU} J \mathrm{JK}}$ & $\mathrm{T}$ & $\mathrm{C}$ & $\mathrm{T}$ & $\mathrm{G}$ & $\mathrm{C}$ & $\mathrm{G}$ & $\mathrm{C}$ & $\mathrm{A}$ & $\mathrm{G}$ & $\mathrm{T}$ & $\mathrm{A}$ & $\mathrm{G}$ & $\mathrm{G}$ & $\mathrm{C}$ & $\mathrm{G}$ & $\mathrm{A}$ & $\mathrm{G}$ \\
\hline $\begin{array}{c}\text { Europ } \\
\mathrm{e}\end{array}$ & $\begin{array}{c}\text { Chuvas } \\
\mathrm{h}\end{array}$ & $\underset{4064}{\text { CHU_JK }}$ & $\mathrm{T}$ & $\mathrm{C}$ & $\mathrm{T}$ & $\mathrm{G}$ & $\mathrm{C}$ & $\mathrm{G}$ & $\mathrm{C}$ & $\mathrm{A}$ & $\mathrm{G}$ & $\mathrm{T}$ & $\mathrm{A}$ & $\mathrm{G}$ & $\mathrm{G}$ & $\mathrm{C}$ & $\mathrm{G}$ & $\mathrm{A}$ & $\bar{G}$ \\
\hline
\end{tabular}

John Wiley 37 Sons, Inc. 


\begin{tabular}{|c|c|c|c|c|c|c|c|c|c|c|c|c|c|c|c|c|c|c|c|}
\hline $\begin{array}{c}\text { Europ } \\
\mathrm{e}\end{array}$ & $\begin{array}{c}\begin{array}{c}\text { Chuvas } \\
\mathrm{h}\end{array} \\
\end{array}$ & $\begin{array}{c}\text { CHU_JK } \\
4065\end{array}$ & $\mathrm{~T}$ & $\mathrm{C}$ & $\mathrm{T}$ & $\mathrm{G}$ & $\mathrm{C}$ & $\mathrm{G}$ & $\mathrm{C}$ & $\mathrm{A}$ & $\mathrm{G}$ & $\mathrm{T}$ & $\mathrm{A}$ & $\mathrm{G}$ & $\mathrm{G}$ & $\mathrm{C}$ & $\mathrm{G}$ & $\mathrm{A}$ & $\bar{G}$ \\
\hline $\begin{array}{c}\text { Europ } \\
\mathrm{e}\end{array}$ & $\begin{array}{c}\text { Chuvas } \\
\text { h }\end{array}$ & $\underset{4065}{\text { CHU_JK }}$ & $\mathrm{T}$ & $\mathrm{C}$ & $\mathrm{T}$ & $\mathrm{G}$ & $\mathrm{C}$ & G & $\mathrm{C}$ & A & $\mathrm{G}$ & $\mathrm{T}$ & $\mathrm{A}$ & $\mathrm{G}$ & $\mathrm{G}$ & $\mathrm{C}$ & $\mathrm{G}$ & $\mathrm{A}$ & $\mathrm{G}$ \\
\hline $\begin{array}{c}\text { Europ } \\
\mathrm{e}\end{array}$ & $\begin{array}{c}\text { Chuvas } \\
\mathrm{h}\end{array}$ & $\begin{array}{c}\text { CHU_JK } \\
4066\end{array}$ & $\bar{T}$ & $\mathrm{C}$ & $\mathrm{T}$ & $\mathrm{G}$ & $\mathrm{C}$ & $\mathrm{G}$ & $\mathrm{C}$ & $\mathrm{A}$ & $\mathrm{G}$ & $\mathrm{T}$ & $\mathrm{A}$ & $\mathrm{G}$ & $\mathrm{G}$ & $\mathrm{C}$ & $\mathrm{G}$ & $\mathrm{A}$ & $\bar{G}$ \\
\hline $\begin{array}{c}\text { Europ } \\
\mathrm{e}\end{array}$ & \begin{tabular}{|c|c}
$\begin{array}{c}\text { Chuvas } \\
\mathrm{h}\end{array}$ \\
\end{tabular} & $\begin{array}{c}\text { CHU_JK } \\
4066 \\
\end{array}$ & $\mathrm{~T}$ & $\mathrm{C}$ & $\mathrm{T}$ & $\mathrm{G}$ & $\mathrm{C}$ & $\mathrm{G}$ & $\mathrm{C}$ & $\mathrm{A}$ & $\mathrm{G}$ & $\mathrm{T}$ & $\mathrm{A}$ & G & $\mathrm{G}$ & $\mathrm{C}$ & $\mathrm{G}$ & $\mathrm{A}$ & $\bar{G}$ \\
\hline $\begin{array}{c}\text { Europ } \\
\mathrm{e}\end{array}$ & $\begin{array}{c}\text { Chuvas } \\
\mathrm{h}\end{array}$ & $\underset{4067}{\text { CHU_JK }}$ & $\mathrm{T}$ & $\mathrm{C}$ & $\mathrm{T}$ & $\mathrm{G}$ & $\mathrm{C}$ & $\mathrm{G}$ & $\mathrm{C}$ & $\bar{A}$ & $\mathrm{G}$ & $\mathrm{T}$ & $\mathrm{A}$ & $\mathrm{G}$ & $\mathrm{G}$ & $\mathrm{C}$ & $\mathrm{G}$ & $\mathrm{A}$ & $\bar{G}$ \\
\hline $\begin{array}{c}\text { Europ } \\
\mathrm{e}\end{array}$ & $\begin{array}{c}\text { Chuvas } \\
\text { h }\end{array}$ & $\underset{4067}{\text { CHU_JK }}$ & $\mathrm{T}$ & $\mathrm{C}$ & $\mathrm{T}$ & $\mathrm{G}$ & $\mathrm{C}$ & $\mathrm{G}$ & $\mathrm{C}$ & $\mathrm{A}$ & $\mathrm{G}$ & $\mathrm{T}$ & $\mathrm{A}$ & $\mathrm{G}$ & $\mathrm{G}$ & $\mathrm{C}$ & $\mathrm{G}$ & $\mathrm{A}$ & $\bar{G}$ \\
\hline $\begin{array}{c}\text { Europ } \\
\mathrm{e}\end{array}$ & $\begin{array}{c}\text { Chuvas } \\
\mathrm{h}\end{array}$ & $\begin{array}{c}\text { CHU_JK } \\
4068\end{array}$ & $\mathrm{~T}$ & $\mathrm{C}$ & $\mathrm{T}$ & $\mathrm{G}$ & $\bar{C}$ & $\mathrm{G}$ & $\mathrm{C}$ & $\mathrm{A}$ & $\mathrm{G}$ & $\mathrm{T}$ & $\mathrm{A}$ & $\mathrm{G}$ & $\mathrm{G}$ & $\mathrm{C}$ & $\mathrm{G}$ & $\mathrm{A}$ & $\mathrm{G}$ \\
\hline $\begin{array}{c}\text { Europ } \\
\mathrm{e}\end{array}$ & $\begin{array}{c}\text { Chuvas } \\
\mathrm{h}\end{array}$ & $\underset{4068}{\mathrm{CHU} J \mathrm{JK}}$ & $\mathrm{T}$ & $\mathrm{C}$ & $\mathrm{T}$ & $\mathrm{G}$ & $\mathrm{C}$ & $\mathrm{G}$ & $\mathrm{C}$ & $\mathrm{A}$ & $\mathrm{G}$ & $\mathrm{T}$ & $\mathrm{A}$ & $\mathrm{G}$ & $\mathrm{G}$ & $\mathrm{C}$ & $\mathrm{G}$ & $\mathrm{A}$ & $\mathrm{G}$ \\
\hline $\begin{array}{c}\text { Europ } \\
\mathrm{e}\end{array}$ & $\begin{array}{c}\text { Chuvas } \\
\mathrm{h}\end{array}$ & $\begin{array}{c}\text { CHU_JK } \\
4069\end{array}$ & $\mathrm{~T}$ & $\mathrm{C}$ & $\mathrm{T}$ & $\mathrm{G}$ & $\mathrm{C}$ & G & $\mathrm{C}$ & $\mathrm{A}$ & $\mathrm{G}$ & $\mathrm{T}$ & $\mathrm{A}$ & $\mathrm{G}$ & $\mathrm{G}$ & $\mathrm{C}$ & $\mathrm{G}$ & $\mathrm{A}$ & $\mathrm{G}$ \\
\hline $\begin{array}{l}\text { Europ } \\
\mathrm{e}\end{array}$ & $\begin{array}{c}\text { Chuvas } \\
\mathrm{h}\end{array}$ & $\begin{array}{c}\text { CHU_JK } \\
4069\end{array}$ & $\mathrm{~T}$ & $\mathrm{C}$ & $\mathrm{T}$ & $\mathrm{G}$ & $\mathrm{C}$ & $G$ & $\mathrm{C}$ & $\mathrm{A}$ & $\mathrm{G}$ & $\mathrm{T}$ & $\mathrm{A}$ & G & $\mathrm{G}$ & $\mathrm{C}$ & $G$ & $\mathrm{~A}$ & $\bar{G}$ \\
\hline $\begin{array}{c}\text { Europ } \\
\mathrm{e}\end{array}$ & $\begin{array}{c}\text { Chuvas } \\
\mathrm{h}\end{array}$ & $\begin{array}{c}\text { CHU_JK } \\
4070\end{array}$ & $\mathrm{~T}$ & $\mathrm{C}$ & $\mathrm{T}$ & $\mathrm{G}$ & $\mathrm{C}$ & $\mathrm{G}$ & $\mathrm{C}$ & $\mathrm{A}$ & $\mathrm{G}$ & $\mathrm{T}$ & $\mathrm{A}$ & $\mathrm{G}$ & $\mathrm{G}$ & $\mathrm{C}$ & $\mathrm{G}$ & $\overline{\mathrm{A}}$ & $\bar{G}$ \\
\hline $\begin{array}{c}\text { Europ } \\
\mathrm{e}\end{array}$ & $\begin{array}{c}\text { Chuvas } \\
\mathrm{h}\end{array}$ & $\underset{4070}{\text { CHU_JK }}$ & $\mathrm{T}$ & $\mathrm{C}$ & $\mathrm{T}$ & $\mathrm{G}$ & $\mathrm{C}$ & $\mathrm{G}$ & $\mathrm{T}$ & $\mathrm{A}$ & $\mathrm{G}$ & $\mathrm{T}$ & $\mathrm{A}$ & $\mathrm{G}$ & $\mathrm{G}$ & $\mathrm{C}$ & $\mathrm{G}$ & $\mathrm{A}$ & $\mathrm{G}$ \\
\hline $\begin{array}{l}\text { Europ } \\
\mathrm{e}\end{array}$ & $\begin{array}{c}\text { Chuvas } \\
\text { h }\end{array}$ & $\begin{array}{c}\text { CHU_JK } \\
4071\end{array}$ & $\mathrm{~T}$ & $\bar{C}$ & $\mathrm{~T}$ & $\mathrm{G}$ & $\bar{C}$ & $\mathrm{G}$ & $\mathrm{C}$ & $\mathrm{A}$ & $\mathrm{G}$ & $\mathrm{T}$ & $\mathrm{A}$ & $\bar{G}$ & $\mathrm{G}$ & $\mathrm{C}$ & $\mathrm{G}$ & $\mathrm{A}$ & $\overline{\mathrm{G}}$ \\
\hline $\begin{array}{c}\text { Europ } \\
\mathrm{e}\end{array}$ & $\begin{array}{c}\text { Chuvas } \\
\mathrm{h}\end{array}$ & $\begin{array}{c}\text { CHU_JK } \\
4071\end{array}$ & $\mathrm{~T}$ & $\mathrm{C}$ & $\mathrm{T}$ & $\mathrm{G}$ & $\mathrm{C}$ & $\mathrm{G}$ & $\mathrm{C}$ & $\mathrm{A}$ & $\mathrm{G}$ & $\mathrm{T}$ & $\mathrm{A}$ & $\mathrm{G}$ & $\mathrm{G}$ & $\mathrm{C}$ & $\mathrm{G}$ & $\overline{\mathrm{A}}$ & $\bar{G}$ \\
\hline $\begin{array}{c}\text { Europ } \\
\mathrm{e}\end{array}$ & $\begin{array}{c}\text { Chuvas } \\
\mathrm{h}\end{array}$ & $\begin{array}{c}\text { CHU_JK } \\
4072\end{array}$ & $\mathrm{~T}$ & $\mathrm{C}$ & $\mathrm{T}$ & $\mathrm{G}$ & $\mathrm{C}$ & $\mathrm{G}$ & $\mathrm{C}$ & $\overline{\mathrm{A}}$ & $\mathrm{G}$ & $\mathrm{T}$ & $\mathrm{A}$ & $\mathrm{G}$ & $\mathrm{G}$ & $\mathrm{C}$ & $\mathrm{G}$ & $\mathrm{A}$ & $\bar{G}$ \\
\hline $\begin{array}{c}\text { Europ } \\
\mathrm{e}\end{array}$ & $\begin{array}{c}\text { Chuvas } \\
\text { h }\end{array}$ & $\begin{array}{c}\text { CHU_JK } \\
4072\end{array}$ & $\mathrm{~T}$ & $\mathrm{C}$ & $\mathrm{T}$ & $\mathrm{G}$ & $\mathrm{C}$ & $\mathrm{G}$ & $\mathrm{C}$ & $\mathrm{A}$ & $\mathrm{G}$ & $\mathrm{T}$ & $\mathrm{A}$ & $\bar{G}$ & $\mathrm{G}$ & $\mathrm{C}$ & $\mathrm{G}$ & $\mathrm{A}$ & $\overline{\mathrm{G}}$ \\
\hline $\begin{array}{c}\text { Europ } \\
\mathrm{e}\end{array}$ & $\begin{array}{c}\text { Chuvas } \\
\mathrm{h}\end{array}$ & $\begin{array}{c}\text { CHU_JK } \\
4073\end{array}$ & $\mathrm{~T}$ & $\mathrm{C}$ & $\mathrm{T}$ & $\mathrm{G}$ & $\bar{C}$ & $\mathrm{G}$ & $\mathrm{C}$ & $\mathrm{A}$ & $\mathrm{G}$ & $\mathrm{T}$ & $\bar{A}$ & $\mathrm{G}$ & $\mathrm{G}$ & $\mathrm{C}$ & $\mathrm{G}$ & $\overline{\mathrm{A}}$ & $\bar{G}$ \\
\hline $\begin{array}{c}\text { Europ } \\
\mathrm{e}\end{array}$ & $\begin{array}{c}\text { Chuvas } \\
\mathrm{h}\end{array}$ & $\underset{4073}{\mathrm{CHU} J \mathrm{JK}}$ & $\mathrm{T}$ & $\mathrm{C}$ & $\mathrm{T}$ & $\mathrm{G}$ & $\mathrm{C}$ & $\mathrm{G}$ & $\mathrm{C}$ & $\mathrm{A}$ & $\mathrm{G}$ & $\mathrm{T}$ & $\mathrm{A}$ & $\mathrm{G}$ & $\mathrm{G}$ & $\mathrm{C}$ & $\mathrm{G}$ & $\mathrm{A}$ & $\bar{G}$ \\
\hline $\begin{array}{c}\text { Europ } \\
\mathrm{e}\end{array}$ & $\begin{array}{c}\text { Chuvas } \\
\mathrm{h}\end{array}$ & $\underset{4074}{\text { CHU_JK }}$ & $\mathrm{T}$ & $\mathrm{C}$ & $\mathrm{T}$ & $\mathrm{G}$ & $\mathrm{C}$ & $\mathrm{G}$ & $\mathrm{C}$ & $\mathrm{A}$ & $\mathrm{G}$ & $\mathrm{T}$ & $\mathrm{A}$ & $\mathrm{G}$ & $\mathrm{G}$ & $\mathrm{C}$ & $\mathrm{G}$ & A & $\bar{G}$ \\
\hline $\begin{array}{c}\text { Europ } \\
\mathrm{e}\end{array}$ & $\begin{array}{c}\text { Chuvas } \\
\mathrm{h}\end{array}$ & $\begin{array}{c}\text { CHU_JK } \\
4074\end{array}$ & $\mathrm{~T}$ & $\mathrm{C}$ & $\mathrm{T}$ & G & $\mathrm{C}$ & $\mathrm{G}$ & $\mathrm{C}$ & $\mathrm{A}$ & $\mathrm{G}$ & $\mathrm{T}$ & $\mathrm{A}$ & $\mathrm{G}$ & $\mathrm{G}$ & $\mathrm{C}$ & $\mathrm{G}$ & $\mathrm{A}$ & $\mathrm{G}$ \\
\hline $\begin{array}{c}\text { Europ } \\
\mathrm{e}\end{array}$ & Danes & $\begin{array}{c}\text { DAN_BK } \\
113\end{array}$ & $\mathrm{~T}$ & $\mathrm{C}$ & $\mathrm{T}$ & $\mathrm{G}$ & $\bar{C}$ & $\mathrm{G}$ & $\bar{C}$ & A & $\mathrm{G}$ & $\mathrm{T}$ & $\mathrm{A}$ & $\mathrm{G}$ & $\mathrm{G}$ & $\mathrm{C}$ & $\mathrm{G}$ & A & $\mathrm{G}$ \\
\hline $\begin{array}{c}\text { Europ } \\
\mathrm{e}\end{array}$ & Danes & $\begin{array}{c}\text { DAN_BK } \\
113\end{array}$ & $\mathrm{~T}$ & $\mathrm{C}$ & $\mathrm{T}$ & $\mathrm{G}$ & $\mathrm{C}$ & $\mathrm{G}$ & $\mathrm{T}$ & $\mathrm{A}$ & $\mathrm{G}$ & $\mathrm{T}$ & $\mathrm{A}$ & $\mathrm{G}$ & $\mathrm{G}$ & $\mathrm{C}$ & $\mathrm{G}$ & $\mathrm{A}$ & $\mathrm{G}$ \\
\hline $\begin{array}{c}\text { Europ } \\
\mathrm{e}\end{array}$ & Danes & $\begin{array}{c}\text { DAN_BK } \\
116 \\
\end{array}$ & $\mathrm{~T}$ & $\mathrm{C}$ & $\mathrm{T}$ & $\mathrm{G}$ & $\mathrm{C}$ & G & $\mathrm{T}$ & $\mathrm{A}$ & $\mathrm{G}$ & $\mathrm{T}$ & $\mathrm{A}$ & $\mathrm{G}$ & $\mathrm{G}$ & $\mathrm{C}$ & $\mathrm{G}$ & $\mathrm{A}$ & $\mathrm{G}$ \\
\hline
\end{tabular}

\author{
John Wiley 38 Sons, Inc.
}




\begin{tabular}{|c|c|c|c|c|c|c|c|c|c|c|c|c|c|c|c|c|c|c|c|}
\hline $\begin{array}{c}\text { Europ } \\
\mathrm{e}\end{array}$ & Danes & $\begin{array}{c}\text { DAN_BK } \\
116\end{array}$ & $\mathrm{~T}$ & $\mathrm{C}$ & $\mathrm{T}$ & $\mathrm{G}$ & $\mathrm{C}$ & $\mathrm{G}$ & $\mathrm{T}$ & $\mathrm{A}$ & $\mathrm{G}$ & $\mathrm{T}$ & $\overline{\mathrm{A}}$ & $\mathrm{G}$ & $\mathrm{G}$ & $\mathrm{C}$ & $\mathrm{G}$ & $\mathrm{A}$ & $\mathrm{G}$ \\
\hline $\begin{array}{c}\text { Europ } \\
\mathrm{e}\end{array}$ & Danes & $\begin{array}{c}\text { DAN_BK } \\
117\end{array}$ & $\mathrm{~T}$ & C & $\mathrm{T}$ & G & $\mathrm{C}$ & $\mathrm{G}$ & $\mathrm{T}$ & $\mathrm{A}$ & $\mathrm{G}$ & $\mathrm{T}$ & $\mathrm{A}$ & $\mathrm{G}$ & $\mathrm{G}$ & $\mathrm{C}$ & G & $\mathrm{A}$ & $\mathrm{G}$ \\
\hline $\begin{array}{c}\text { Europ } \\
\mathrm{e}\end{array}$ & Danes & $\begin{array}{c}\text { DAN_BK } \\
117\end{array}$ & $\mathrm{~T}$ & $\mathrm{C}$ & $\mathrm{T}$ & $\mathrm{G}$ & $\mathrm{C}$ & $\mathrm{G}$ & $\bar{T}$ & $\overline{\mathrm{A}}$ & $\bar{G}$ & $\mathrm{~T}$ & $\bar{A}$ & $\bar{G}$ & $\mathrm{G}$ & $\mathrm{C}$ & $\mathrm{G}$ & $\mathrm{A}$ & $\mathrm{G}$ \\
\hline $\begin{array}{c}\text { Europ } \\
\mathrm{e}\end{array}$ & Danes & $\begin{array}{c}\text { DAN_BK } \\
119 \\
\end{array}$ & $\mathrm{~T}$ & $\mathrm{C}$ & $\mathrm{T}$ & $\mathrm{G}$ & $\mathrm{C}$ & $\mathrm{G}$ & $\mathrm{C}$ & $\mathrm{A}$ & $\mathrm{G}$ & $\mathrm{T}$ & $\mathrm{A}$ & $\mathrm{G}$ & $\mathrm{G}$ & $\mathrm{C}$ & $\mathrm{G}$ & $\mathrm{A}$ & $\mathrm{G}$ \\
\hline $\begin{array}{c}\text { Europ } \\
\mathrm{e}\end{array}$ & Danes & $\begin{array}{c}\text { DAN_BK } \\
119\end{array}$ & $\mathrm{~T}$ & $\mathrm{C}$ & $\mathrm{T}$ & $\mathrm{G}$ & $\mathrm{C}$ & $\mathrm{G}$ & $\mathrm{C}$ & $\mathrm{A}$ & $\mathrm{G}$ & $\mathrm{T}$ & $\mathrm{A}$ & $\mathrm{G}$ & $\mathrm{G}$ & $\mathrm{C}$ & $\mathrm{G}$ & $\mathrm{A}$ & $\bar{G}$ \\
\hline $\begin{array}{c}\text { Europ } \\
\mathrm{e}\end{array}$ & Danes & $\begin{array}{c}\text { DAN_BK } \\
120\end{array}$ & $\mathrm{~T}$ & $\mathrm{C}$ & $\mathrm{T}$ & G & $\mathrm{C}$ & $\mathrm{G}$ & $\mathrm{C}$ & $\mathrm{A}$ & $G$ & $\mathrm{~T}$ & $\mathrm{~A}$ & $\mathrm{G}$ & $G$ & $\mathrm{C}$ & $\mathrm{G}$ & $\mathrm{A}$ & $\mathrm{G}$ \\
\hline $\begin{array}{c}\text { Europ } \\
\mathrm{e}\end{array}$ & Danes & $\begin{array}{c}\text { DAN_BK } \\
120\end{array}$ & $\mathrm{~T}$ & $\mathrm{C}$ & $\mathrm{T}$ & $\mathrm{G}$ & $\mathrm{C}$ & $\mathrm{G}$ & $\mathrm{T}$ & $\mathrm{A}$ & $\mathrm{G}$ & $\mathrm{T}$ & $\bar{A}$ & $\mathrm{G}$ & $\mathrm{G}$ & $\mathrm{C}$ & $\mathrm{G}$ & $\bar{A}$ & $\bar{G}$ \\
\hline $\begin{array}{c}\text { Europ } \\
\mathrm{e}\end{array}$ & Danes & $\begin{array}{c}\text { DAN_BK } \\
124\end{array}$ & $\mathrm{~T}$ & $\mathrm{C}$ & $\mathrm{T}$ & $\mathrm{G}$ & $\mathrm{C}$ & $\mathrm{G}$ & C & A & $\mathrm{G}$ & $\mathrm{T}$ & $\mathrm{A}$ & $\mathrm{G}$ & $\mathrm{G}$ & $\mathrm{C}$ & $\mathrm{G}$ & $\mathrm{A}$ & $\bar{G}$ \\
\hline $\begin{array}{c}\text { Europ } \\
\mathrm{e}\end{array}$ & Danes & $\begin{array}{c}\text { DAN_BK } \\
124 \\
\end{array}$ & $\mathrm{~T}$ & $\mathrm{C}$ & $\mathrm{T}$ & $G$ & $\mathrm{C}$ & $\mathrm{G}$ & $\mathrm{C}$ & $\mathrm{A}$ & $\mathrm{G}$ & $\mathrm{T}$ & A & $\mathrm{G}$ & G & $\mathrm{C}$ & G & $\mathrm{A}$ & $\mathrm{G}$ \\
\hline $\begin{array}{c}\text { Europ } \\
\mathrm{e}\end{array}$ & Danes & $\begin{array}{c}\text { DAN_BK } \\
127\end{array}$ & $\mathrm{~T}$ & $\mathrm{C}$ & $\mathrm{T}$ & $\mathrm{G}$ & $\mathrm{C}$ & $\mathrm{G}$ & $\mathrm{T}$ & $\mathrm{A}$ & $\mathrm{G}$ & $\mathrm{T}$ & $\mathrm{A}$ & $\mathrm{G}$ & $\mathrm{G}$ & $\mathrm{C}$ & $\mathrm{G}$ & $\mathrm{A}$ & G \\
\hline $\begin{array}{c}\text { Europ } \\
e\end{array}$ & Danes & $\begin{array}{c}\text { DAN_BK } \\
127\end{array}$ & $\mathrm{~T}$ & $\mathrm{C}$ & $\bar{T}$ & $\mathrm{G}$ & $\mathrm{C}$ & $\bar{G}$ & $\mathrm{~T}$ & $\mathrm{~A}$ & $\mathrm{G}$ & $\mathrm{T}$ & $\mathrm{A}$ & $\mathrm{G}$ & $\mathrm{G}$ & $\mathrm{C}$ & $\mathrm{G}$ & $\mathrm{A}$ & $\overline{\mathrm{G}}$ \\
\hline $\begin{array}{c}\text { Europ } \\
\mathrm{e}\end{array}$ & Danes & $\begin{array}{c}\text { DAN_BK } \\
141 \\
\end{array}$ & $\mathrm{~T}$ & $\mathrm{C}$ & $\mathrm{T}$ & G & $\mathrm{C}$ & $\mathrm{G}$ & $\mathrm{C}$ & $\overline{\mathrm{A}}$ & $G$ & $\mathrm{~T}$ & A & $\mathrm{G}$ & $\mathrm{G}$ & $\mathrm{C}$ & $\mathrm{G}$ & $\mathrm{A}$ & $\mathrm{G}$ \\
\hline $\begin{array}{c}\text { Europ } \\
\mathrm{e}\end{array}$ & Danes & $\begin{array}{c}\text { DAN_BK } \\
141\end{array}$ & $\mathrm{~T}$ & $\mathrm{C}$ & $\mathrm{T}$ & $\mathrm{G}$ & $\mathrm{C}$ & $\mathrm{G}$ & $\bar{C}$ & $\mathrm{~A}$ & $\mathrm{G}$ & $\mathrm{T}$ & $\bar{A}$ & $\mathrm{G}$ & $\mathrm{G}$ & $\mathrm{C}$ & $\mathrm{G}$ & $\mathrm{A}$ & $\mathrm{G}$ \\
\hline $\begin{array}{c}\text { Europ } \\
\mathrm{e}\end{array}$ & Danes & $\begin{array}{c}\text { DAN_BK } \\
144\end{array}$ & $\mathrm{~T}$ & $\mathrm{C}$ & $\mathrm{T}$ & $\mathrm{G}$ & $\mathrm{C}$ & $\bar{G}$ & $\mathrm{C}$ & $\mathrm{A}$ & $G$ & $\mathrm{~T}$ & $\mathrm{~A}$ & $\mathrm{G}$ & $\bar{G}$ & $\mathrm{C}$ & $\mathrm{G}$ & $\mathrm{A}$ & $\overline{\mathrm{G}}$ \\
\hline $\begin{array}{c}\text { Europ } \\
\mathrm{e}\end{array}$ & Danes & $\begin{array}{c}\text { DAN_BK } \\
144 \\
\end{array}$ & $\mathrm{~T}$ & $\mathrm{C}$ & $\mathrm{T}$ & $\mathrm{G}$ & $\mathrm{C}$ & $\bar{G}$ & $\bar{T}$ & $\overline{\mathrm{A}}$ & $\bar{G}$ & $T$ & $\bar{A}$ & $\mathrm{G}$ & $\mathrm{G}$ & $\mathrm{C}$ & $\mathrm{G}$ & $\mathrm{A}$ & $\mathrm{G}$ \\
\hline $\begin{array}{c}\text { Europ } \\
\mathrm{e}\end{array}$ & Danes & $\begin{array}{c}\text { DAN_BK } \\
146\end{array}$ & $\mathrm{~T}$ & $\mathrm{C}$ & $\mathrm{T}$ & $\mathrm{G}$ & $\mathrm{C}$ & $\mathrm{G}$ & $\mathrm{C}$ & $\mathrm{A}$ & $\mathrm{G}$ & $\mathrm{C}$ & $\bar{A}$ & $\mathrm{G}$ & $\mathrm{G}$ & $\mathrm{C}$ & $\mathrm{G}$ & $\mathrm{A}$ & $\mathrm{G}$ \\
\hline $\begin{array}{c}\text { Europ } \\
\mathrm{e}\end{array}$ & Danes & $\begin{array}{c}\text { DAN_BK } \\
146\end{array}$ & $\mathrm{~T}$ & $\mathrm{C}$ & $\mathrm{T}$ & $\mathrm{G}$ & $\mathrm{C}$ & $\bar{G}$ & $\mathrm{C}$ & $\mathrm{A}$ & $\mathrm{G}$ & $\mathrm{T}$ & $\bar{A}$ & $\mathrm{G}$ & $\mathrm{G}$ & $\mathrm{C}$ & $\mathrm{G}$ & $\mathrm{A}$ & $\bar{G}$ \\
\hline $\begin{array}{c}\text { Europ } \\
\mathrm{e}\end{array}$ & Danes & $\begin{array}{c}\text { DAN_BK } \\
153 \\
\end{array}$ & $\mathrm{~T}$ & $\mathrm{C}$ & $\mathrm{T}$ & $\mathrm{G}$ & $\mathrm{C}$ & $\mathrm{G}$ & $\mathrm{C}$ & $\overline{\mathrm{A}}$ & $\mathrm{G}$ & $\mathrm{T}$ & A & $\mathrm{G}$ & $\mathrm{G}$ & $\mathrm{C}$ & $\mathrm{G}$ & $\mathrm{A}$ & $\mathrm{G}$ \\
\hline $\begin{array}{c}\text { Europ } \\
\mathrm{e}\end{array}$ & Danes & $\begin{array}{c}\text { DAN_BK } \\
153\end{array}$ & $\mathrm{~T}$ & $\mathrm{C}$ & $\mathrm{T}$ & $\mathrm{G}$ & $\mathrm{C}$ & $\mathrm{G}$ & $\mathrm{C}$ & $\mathrm{A}$ & $\mathrm{G}$ & $\mathrm{T}$ & $\overline{\mathrm{A}}$ & $\mathrm{G}$ & G & $\mathrm{C}$ & $\mathrm{G}$ & $\mathrm{A}$ & G \\
\hline $\begin{array}{c}\text { Europ } \\
\mathrm{e}\end{array}$ & Danes & $\begin{array}{c}\text { DAN_BK } \\
157\end{array}$ & $\mathrm{~T}$ & $\mathrm{C}$ & $\mathrm{T}$ & $\mathrm{G}$ & $\mathrm{C}$ & $\mathrm{G}$ & $\mathrm{C}$ & $\mathrm{A}$ & $\mathrm{G}$ & $\mathrm{T}$ & $\mathrm{A}$ & $\mathrm{G}$ & G & $\mathrm{C}$ & $\mathrm{G}$ & $\mathrm{A}$ & $\mathrm{G}$ \\
\hline $\begin{array}{c}\text { Europ } \\
\mathrm{e}\end{array}$ & Danes & $\begin{array}{c}\text { DAN_BK } \\
157 \\
\end{array}$ & $\mathrm{~T}$ & $\mathrm{C}$ & $\mathrm{T}$ & $\mathrm{G}$ & $\mathrm{C}$ & $\mathrm{G}$ & $\mathrm{T}$ & A & $\mathrm{G}$ & $\mathrm{T}$ & $\bar{A}$ & $\mathrm{G}$ & G & $\mathrm{C}$ & $\mathrm{G}$ & $\mathrm{A}$ & $\mathrm{G}$ \\
\hline $\begin{array}{c}\text { Europ } \\
\mathrm{e}\end{array}$ & Danes & $\begin{array}{c}\text { DAN_BK } \\
160\end{array}$ & $\mathrm{~T}$ & $\mathrm{C}$ & $\mathrm{T}$ & $\mathrm{G}$ & $\mathrm{C}$ & $\mathrm{G}$ & $\mathrm{C}$ & $\bar{A}$ & $\mathrm{G}$ & $\mathrm{T}$ & A & $\mathrm{G}$ & $\bar{G}$ & $\mathrm{C}$ & $\mathrm{G}$ & $\mathrm{A}$ & $\mathrm{G}$ \\
\hline $\begin{array}{c}\text { Europ } \\
\mathrm{e}\end{array}$ & Danes & $\begin{array}{c}\text { DAN_BK } \\
160\end{array}$ & $\mathrm{~T}$ & $\mathrm{C}$ & $\mathrm{T}$ & $\mathrm{G}$ & $\mathrm{C}$ & $\mathrm{G}$ & $\mathrm{C}$ & $\mathrm{A}$ & $\mathrm{G}$ & $\mathrm{T}$ & $\mathrm{A}$ & $\mathrm{G}$ & $\mathrm{G}$ & $\mathrm{C}$ & $\mathrm{G}$ & $\mathrm{A}$ & G \\
\hline
\end{tabular}

John Wiley 39 \& Sons, Inc. 


\begin{tabular}{|c|c|c|c|c|c|c|c|c|c|c|c|c|c|c|c|c|c|c|c|}
\hline $\begin{array}{c}\text { Europ } \\
\mathrm{e}\end{array}$ & Danes & $\begin{array}{c}\text { DAN_BK } \\
171\end{array}$ & $\mathrm{~T}$ & $\mathrm{C}$ & $\mathrm{T}$ & G & $\mathrm{C}$ & $\mathrm{G}$ & $\mathrm{C}$ & $\mathrm{A}$ & $\mathrm{G}$ & $\mathrm{T}$ & $\bar{A}$ & $\mathrm{G}$ & $\mathrm{G}$ & $\mathrm{C}$ & $\mathrm{G}$ & $\mathrm{A}$ & $\mathrm{G}$ \\
\hline $\begin{array}{c}\text { Europ } \\
\mathrm{e}\end{array}$ & Danes & $\begin{array}{c}\text { DAN_BK } \\
171\end{array}$ & $\mathrm{~T}$ & $\mathrm{C}$ & $\mathrm{T}$ & $\bar{G}$ & $\mathrm{C}$ & $\mathrm{G}$ & $\mathrm{T}$ & A & $\mathrm{G}$ & $\mathrm{T}$ & $\mathrm{A}$ & $\mathrm{G}$ & $\mathrm{G}$ & $\mathrm{C}$ & $\mathrm{G}$ & $\mathrm{A}$ & $\mathrm{G}$ \\
\hline $\begin{array}{c}\text { Europ } \\
\mathrm{e}\end{array}$ & Danes & $\begin{array}{c}\text { DAN_BK } \\
172 \\
\end{array}$ & $\bar{T}$ & $\mathrm{C}$ & $\mathrm{T}$ & $\bar{G}$ & $\mathrm{C}$ & $\mathrm{G}$ & $\mathrm{C}$ & $\mathrm{A}$ & $\mathrm{G}$ & $\mathrm{T}$ & $\mathrm{A}$ & $\mathrm{G}$ & $\mathrm{G}$ & $\mathrm{C}$ & $\mathrm{G}$ & $\mathrm{A}$ & $\mathrm{G}$ \\
\hline $\begin{array}{c}\text { Europ } \\
\mathrm{e}\end{array}$ & Danes & $\begin{array}{c}\text { DAN_BK } \\
172 \\
\end{array}$ & $\mathrm{~T}$ & $\mathrm{C}$ & $\mathrm{T}$ & G & $\mathrm{C}$ & $\mathrm{G}$ & $\mathrm{T}$ & $\mathrm{A}$ & $\mathrm{G}$ & $\mathrm{T}$ & $\mathrm{A}$ & G & $\mathrm{G}$ & $\mathrm{C}$ & $\mathrm{G}$ & $\mathrm{A}$ & $\mathrm{G}$ \\
\hline $\begin{array}{c}\text { Europ } \\
\mathrm{e}\end{array}$ & Danes & $\begin{array}{c}\text { DAN_BK } \\
180\end{array}$ & $\mathrm{~T}$ & $\mathrm{C}$ & $\mathrm{T}$ & $\bar{G}$ & $\mathrm{C}$ & $\mathrm{G}$ & $\mathrm{C}$ & $\bar{A}$ & $\mathrm{G}$ & $\mathrm{T}$ & $\mathrm{A}$ & $\mathrm{G}$ & $\mathrm{G}$ & $\mathrm{C}$ & $\mathrm{G}$ & $\mathrm{A}$ & $\mathrm{G}$ \\
\hline $\begin{array}{c}\text { Europ } \\
\mathrm{e}\end{array}$ & Danes & $\begin{array}{c}\text { DAN_BK } \\
180\end{array}$ & $\mathrm{~T}$ & $\mathrm{C}$ & $\mathrm{T}$ & $\bar{G}$ & $\mathrm{C}$ & $\mathrm{G}$ & $\mathrm{T}$ & $\mathrm{A}$ & $\mathrm{G}$ & $\mathrm{T}$ & $\mathrm{A}$ & G & $\mathrm{G}$ & $\mathrm{C}$ & $\mathrm{G}$ & $\mathrm{A}$ & $\mathrm{G}$ \\
\hline $\begin{array}{c}\text { Europ } \\
\mathrm{e}\end{array}$ & Danes & $\begin{array}{c}\text { DAN_JK } \\
3330 \\
\end{array}$ & $\mathrm{~T}$ & $\mathrm{C}$ & $\mathrm{T}$ & $\mathrm{G}$ & $\mathrm{C}$ & $\mathrm{G}$ & $\mathrm{C}$ & $\mathrm{A}$ & $\mathrm{G}$ & $\mathrm{T}$ & $\mathrm{A}$ & $\bar{G}$ & $\mathrm{G}$ & $\mathrm{C}$ & $\mathrm{G}$ & $\mathrm{A}$ & $\mathrm{G}$ \\
\hline $\begin{array}{l}\text { Europ } \\
\mathrm{e}\end{array}$ & Danes & $\begin{array}{c}\text { DAN_JK } \\
\mathbf{3 3 3 0}\end{array}$ & $\mathrm{T}$ & $\mathrm{C}$ & $\mathrm{T}$ & G & $\mathrm{C}$ & $\mathrm{G}$ & $\mathrm{T}$ & $\mathrm{A}$ & $\mathrm{G}$ & $\mathrm{T}$ & $\mathrm{A}$ & G & $\mathrm{G}$ & $\mathrm{C}$ & $\mathrm{G}$ & $\mathrm{A}$ & $\mathrm{G}$ \\
\hline $\begin{array}{c}\text { Europ } \\
\mathrm{e}\end{array}$ & Danes & $\begin{array}{c}\text { DAN_JK_KK } \\
\mathbf{3 3 3 1}\end{array}$ & $\mathrm{T}$ & $\mathrm{C}$ & $\mathrm{T}$ & $\mathrm{G}$ & $\mathrm{C}$ & $\mathrm{G}$ & $\mathrm{C}$ & $\mathrm{A}$ & $\mathrm{G}$ & $\mathrm{T}$ & $\mathrm{A}$ & G & $\mathrm{G}$ & $\mathrm{C}$ & $\mathrm{G}$ & A & $\mathrm{G}$ \\
\hline $\begin{array}{l}\text { Europ } \\
\mathrm{e}\end{array}$ & Danes & $\begin{array}{c}\text { DAN_JK } \\
3331\end{array}$ & $\mathrm{~T}$ & $\mathrm{C}$ & $\mathrm{T}$ & G & $\mathrm{C}$ & $\mathrm{G}$ & $\mathrm{C}$ & $\mathrm{A}$ & $\mathrm{G}$ & $\mathrm{T}$ & $\mathrm{A}$ & G & G & $\mathrm{C}$ & $\mathrm{G}$ & $\mathrm{A}$ & $\mathrm{G}$ \\
\hline $\begin{array}{c}\text { Europ } \\
\mathrm{e}\end{array}$ & Danes & $\begin{array}{c}\text { DAN_JK } \\
3332 \\
\end{array}$ & $\mathrm{~T}$ & $\mathrm{C}$ & $\mathrm{T}$ & $\bar{G}$ & $\mathrm{C}$ & $\mathrm{G}$ & $\mathrm{T}$ & $\mathrm{A}$ & $\mathrm{G}$ & $\mathrm{T}$ & $\mathrm{A}$ & G & $\mathrm{G}$ & $\mathrm{C}$ & $\mathrm{G}$ & $\mathrm{A}$ & $\mathrm{G}$ \\
\hline $\begin{array}{c}\text { Europ } \\
\mathrm{e}\end{array}$ & Danes & $\begin{array}{c}\text { DAN_JK } \\
3332\end{array}$ & $\mathrm{~T}$ & $\mathrm{C}$ & $\mathrm{T}$ & G & $\mathrm{C}$ & $\mathrm{G}$ & $\mathrm{T}$ & $\mathrm{A}$ & $\mathrm{G}$ & $\mathrm{T}$ & $\mathrm{A}$ & $\mathrm{G}$ & $\mathrm{G}$ & $\mathrm{C}$ & $\mathrm{G}$ & $\mathrm{A}$ & $\mathrm{G}$ \\
\hline $\begin{array}{l}\text { Europ } \\
\mathrm{e}\end{array}$ & Danes & $\begin{array}{c}\text { DAN_JK } \\
3333\end{array}$ & $\mathrm{~T}$ & $\mathrm{C}$ & $\mathrm{T}$ & $\mathrm{G}$ & $\mathrm{C}$ & $\mathrm{G}$ & $\mathrm{C}$ & $\mathrm{A}$ & $\mathrm{G}$ & $\mathrm{T}$ & $\mathrm{A}$ & $\bar{G}$ & $\mathrm{G}$ & $\mathrm{C}$ & $\mathrm{G}$ & $\mathrm{A}$ & $\bar{G}$ \\
\hline $\begin{array}{c}\text { Europ } \\
\mathrm{e}\end{array}$ & Danes & $\begin{array}{c}\text { DAN_JK } \\
3333\end{array}$ & $\mathrm{~T}$ & $\mathrm{C}$ & $\mathrm{T}$ & G & $\mathrm{C}$ & $\mathrm{G}$ & $\mathrm{T}$ & $\mathrm{A}$ & $\mathrm{G}$ & $\mathrm{T}$ & $\bar{A}$ & $\mathrm{G}$ & $\mathrm{G}$ & $\mathrm{C}$ & $\mathrm{G}$ & $\mathrm{A}$ & $\mathrm{G}$ \\
\hline $\begin{array}{c}\text { Europ } \\
\mathrm{e}\end{array}$ & Danes & $\begin{array}{c}\text { DAN_JK } \\
3334\end{array}$ & $\mathrm{~T}$ & $\mathrm{C}$ & $\mathrm{T}$ & $\bar{G}$ & $\bar{C}$ & $\mathrm{G}$ & $\mathrm{C}$ & $\overline{\mathrm{A}}$ & $\mathrm{G}$ & $\mathrm{T}$ & $\mathrm{A}$ & $\mathrm{G}$ & $\mathrm{G}$ & $\mathrm{C}$ & $\mathrm{G}$ & $\mathrm{A}$ & $\bar{G}$ \\
\hline $\begin{array}{c}\text { Europ } \\
\mathrm{e}\end{array}$ & Danes & $\begin{array}{c}\text { DAN_JK } \\
3334\end{array}$ & $\mathrm{~T}$ & $\mathrm{C}$ & $\mathrm{T}$ & $\mathrm{G}$ & $\mathrm{C}$ & $\mathrm{G}$ & $\mathrm{T}$ & $\mathrm{A}$ & $\mathrm{G}$ & $\mathrm{T}$ & $\mathrm{A}$ & G & $\mathrm{G}$ & $\mathrm{C}$ & $\mathrm{G}$ & $\mathrm{A}$ & $\overline{\mathrm{G}}$ \\
\hline $\begin{array}{c}\text { Europ } \\
\mathrm{e}\end{array}$ & Danes & $\begin{array}{c}\text { DAN_JK } \\
3335 \\
\end{array}$ & $\mathrm{~T}$ & $\mathrm{C}$ & $\mathrm{T}$ & G & $\mathrm{C}$ & $\mathrm{G}$ & $\mathrm{C}$ & $\mathrm{A}$ & $\mathrm{G}$ & $\mathrm{T}$ & $\mathrm{A}$ & $\mathrm{G}$ & $\mathrm{G}$ & $\mathrm{C}$ & $\mathrm{G}$ & $\mathrm{A}$ & $\bar{G}$ \\
\hline $\begin{array}{c}\text { Europ } \\
\mathrm{e}\end{array}$ & Danes & $\begin{array}{c}\text { DAN_JK } \\
3335\end{array}$ & $\mathrm{~T}$ & $\mathrm{C}$ & $\mathrm{T}$ & G & $\mathrm{C}$ & $\mathrm{G}$ & $\mathrm{C}$ & $\mathrm{A}$ & $\mathrm{G}$ & $\mathrm{T}$ & $\mathrm{A}$ & $\mathrm{G}$ & $\mathrm{G}$ & $\mathrm{C}$ & $\mathrm{G}$ & $\mathrm{A}$ & G \\
\hline $\begin{array}{c}\text { Europ } \\
\mathrm{e}\end{array}$ & Danes & $\begin{array}{c}\text { DAN_JK } \\
3336\end{array}$ & $\mathrm{~T}$ & $\mathrm{C}$ & $\mathrm{T}$ & $\mathrm{G}$ & $\mathrm{C}$ & $\mathrm{G}$ & $\mathrm{C}$ & $\mathrm{A}$ & $\mathrm{G}$ & $\mathrm{T}$ & $\mathrm{A}$ & $\mathrm{G}$ & $\mathrm{G}$ & $\mathrm{C}$ & $\mathrm{G}$ & $\mathrm{A}$ & G \\
\hline $\begin{array}{c}\text { Europ } \\
\mathrm{e}\end{array}$ & Danes & $\begin{array}{c}\text { DAN_JK } \\
3336 \\
\end{array}$ & $\mathrm{~T}$ & $\mathrm{C}$ & $\mathrm{T}$ & G & $\mathrm{C}$ & $\mathrm{G}$ & $\mathrm{T}$ & $\mathrm{A}$ & $\mathrm{G}$ & $\mathrm{T}$ & $\mathrm{A}$ & $\mathrm{G}$ & $\mathrm{G}$ & $\mathrm{C}$ & $\mathrm{G}$ & $\mathrm{A}$ & $\mathrm{G}$ \\
\hline
\end{tabular}

\author{
John Wiley ${ }^{4} \&$ Sons, Inc.
}

